\title{
Variation Simulation of Fixtured Assembly Processes for Compliant Structures Using Piecewise-Linear Analysis
}

Michael L. Stewart

Brigham Young University - Provo

Follow this and additional works at: https://scholarsarchive.byu.edu/etd

Part of the Mechanical Engineering Commons

\section{BYU ScholarsArchive Citation}

Stewart, Michael L., "Variation Simulation of Fixtured Assembly Processes for Compliant Structures Using Piecewise-Linear Analysis" (2004). Theses and Dissertations. 195.

https://scholarsarchive.byu.edu/etd/195

This Thesis is brought to you for free and open access by BYU ScholarsArchive. It has been accepted for inclusion in Theses and Dissertations by an authorized administrator of BYU ScholarsArchive. For more information, please contact scholarsarchive@byu.edu, ellen_amatangelo@byu.edu. 


\title{
VARIATION SIMULATION OF FIXTURED ASSEMBLY PROCESSES FOR COMPLIANT STRUCTURES USING PIECEWISE-LINEAR ANALYSIS
}

\author{
by
}

Michael Leon Stewart

A thesis submitted to the faculty of Brigham Young University in partial fulfillment of the requirements for the degree of

Master of Science

Department of Mechanical Engineering

Brigham Young University

December 2004 

Copyright (C) 2004 Michael Leon Stewart

All Rights Reserved 



\title{
BRIGHAM YOUNG UNIVERSITY
}

\section{GRADUATE COMMITTEE APPROVAL}

\author{
of a thesis submitted by \\ Michael Leon Stewart
}

This thesis has been read by each member of the following graduate committee and by a majority vote has been found to be satisfactory.

Date

Date

Date

\author{
Kenneth W. Chase, Chair
}

Steven E. Benzley

Jonathan D. Blotter 



\section{BRIGHAM YOUNG UNIVERSITY}

As chair of the candidate's graduate committee, I have read the thesis of Michael Leon Stewart in it final form and have found that (1) its format, citations, and bibliographical style are consistent and acceptable and fulfill university and department style requirements; (2) its illustrative materials including figure, tables, and charts are in place; and (3) the final manuscript is satisfactory to the graduate committee and is ready for submission to the university library.

Date

Accepted for the Department
Kenneth. W. Chase

Chair, Graduate Committee
Matthew R. Jones

Graduate Coordinator

Accepted for the College

Douglas M. Chabries

Dean, Ira A. Fulton College of Engineering and Technology 



\begin{abstract}
VARIATION SIMULATION OF FIXTURED ASSEMBLY PROCESSES FOR COMPLIANT STRUCTURES USING PIECEWISE-LINEAR ANALYSIS
\end{abstract}

\author{
Michael Leon Stewart \\ Department of Mechanical Engineering \\ Master of Science
}

While variation analysis methods for compliant assemblies are not new, little has been done to include the effects of multi-step, fixtured assembly processes. This thesis introduces a new method to statistically analyze compliant part assembly processes using fixtures. This method, consistent with the FASTA method developed at BYU, yields both a mean and a variant solution. The method, called Piecewise-Linear Elastic Analysis, or PLEA, is developed for predicting the residual stress, deformation and springback variation in compliant assemblies. A comprehensive, step-by-step analysis map is provided. PLEA is validated on a simple, laboratory assembly and a more complex, production assembly. Significant modeling findings are reported as well as the comparison of the analytical to physical results. 



\section{ACKNOWLEDGEMENTS}

I am indebted to many for this paper. I first and foremost thank my charitable wife, Holly, for her continual love and support in all my endeavors. All truth be told; it was for her that I decided to stay and attend graduate school. I thank my wonderful parents, Dale and Marie, for providing me with a learning environment throughout my youth. They have been my greatest teachers. I thank my grandfather, James Robertson, for instilling within me while I was in my youth a curiosity for the sciences. I thank Tom and Wendy Mason for standing in as my parents while I was at school.

Many deserve recognition for their hand in my professional development. I thank my advisor, Dr. Ken Chase, for his help and insights. The field of tolerance analysis has benefited greatly by his knowledge and labors. I also thank Nephi Mourik for introducing me to Finite Element Analysis and trusting in the analysis findings. I also thank the other great (and often patient) educators from whom I have benefited; Pam Oliverio, John Beck, John Blasi, Rich Hoge, Greg Roach and Spencer Magleby.

I would also like to profusely thank Devin and La Nan Bedwell, Darren and Cherish Bedwell, Jim Scribner and Stephanie Miller, Lyman and Liz Pierce, Reuben Erickson, Nate Mathews, and Mike Baxter for their friendships. 

My graduate career and this thesis were made possible by the contributions of the Boeing Company and the ADCATS Consortium and I thank them for their support. I also thank John Heiner and Clint Bybee for their assistance on the CMM and Boeing employee Kelly Nielson for demonstrating the Boeing assembly process.

I testify of the hand of my loving Father in Heaven in the completion of this thesis, and I thank Him greatly for his assistance in leading me to discover that which He already knows. Throughout the development of this thesis, He has guided me from grace to grace (D\&C 93:13), leading me enough to keep me enthused, but not so much that I wasn't provided with the necessary challenges and growth. I am awestruck that neither a sparrow's fall nor my struggles with this thesis escape His notice nor merit His attention. 



\section{$\underline{\text { Table of Contents }}$}

CHAPTER 1： THESIS INTRODUCTION ........................................................ 1

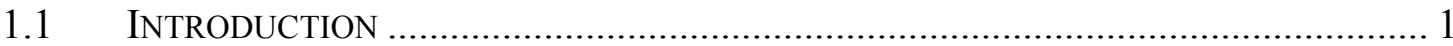

1.1.1 Compliant Assemblies ........................................................................... 1

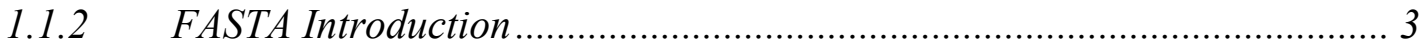

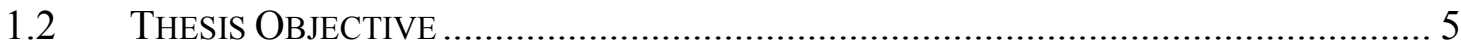

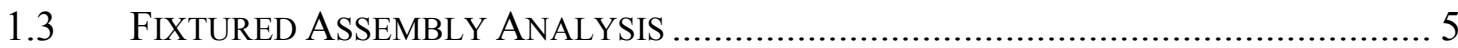

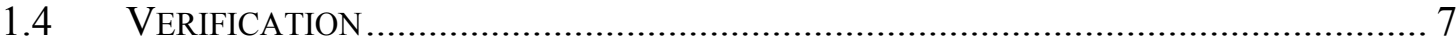

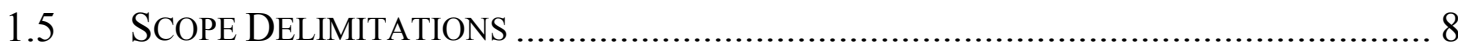

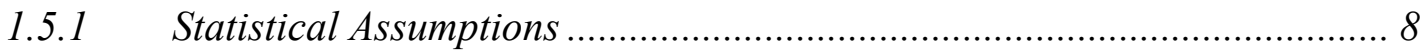

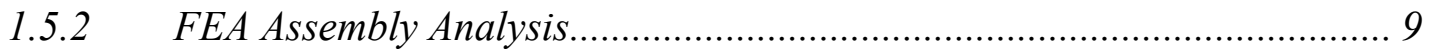

1.5.3 Linear Elastic Assumptions of Components and Assemblies.................... 9

1.5.4 Access to Appropriate Software Packages and Equipment .................... 10

1.5.5 Normal Closure Forces and Moments .................................................. 11

1.5.6 Mean Verification ............................................................................. 11

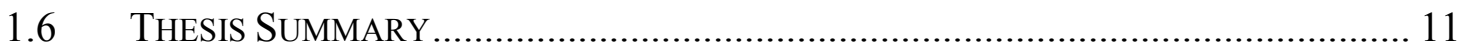

CHAPTER 2： BACKGROUND RESEARCH .................................................. 13

2.1 RIGID BODY TOLERANCE ANALYSIS............................................................. 13

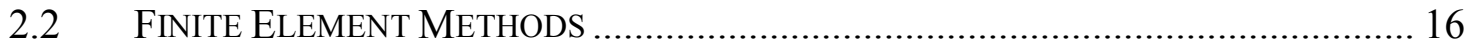


2.3 FLEXIBLE ASSEMBLY VARIATION ANALYSIS ................................................ 17

2.4 Flexible Assembly Statistical Tolerance Analysis .............................. 20

CHAPTER 3: ANALYSIS USING THE FASTA METHOD ..................................... 25

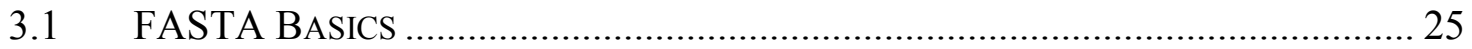

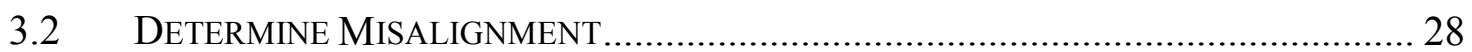

3.2.1 Real Assemblies ............................................................................... 28

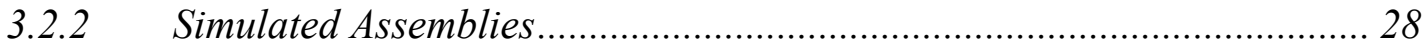

3.3 Finite Element ModeLING OF COMPLIANT PARTS ......................................... 30

3.3.1 Substructuring .................................................................................. 31

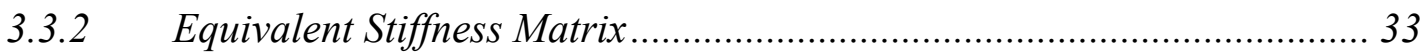

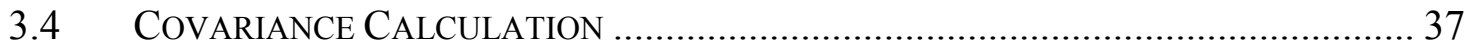

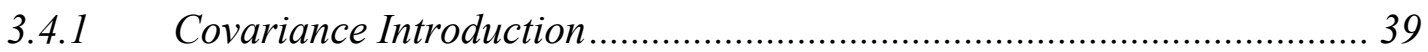

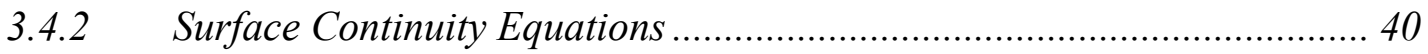

3.4.2.1 The Bézier Curve Method................................................................... 41

3.4.2.2 The Polynomial Method ………………............................................ 41

3.4.2.3 The Frequency Spectrum Method.......................................................... 42

3.4.2.4 The Sinusoidal Method ....................................................................... 44

3.4.2.5 Polynomial Series Methods ……………........................................... 45

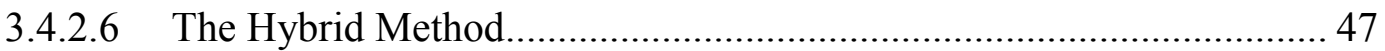

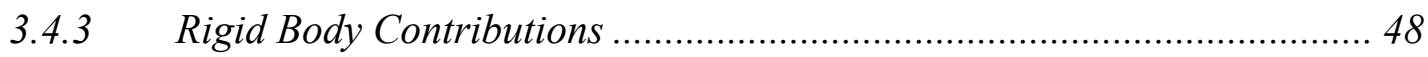

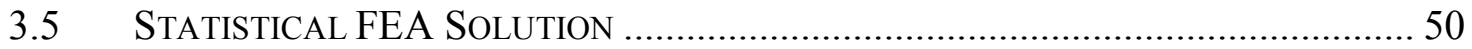

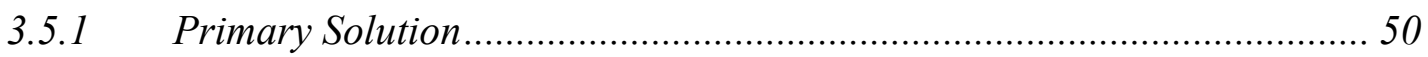

3.5.2 Part Solutions and Post-Processing .................................................. 50 


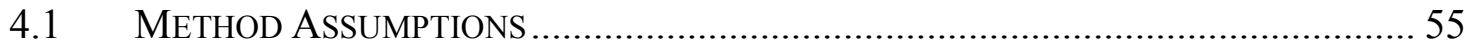

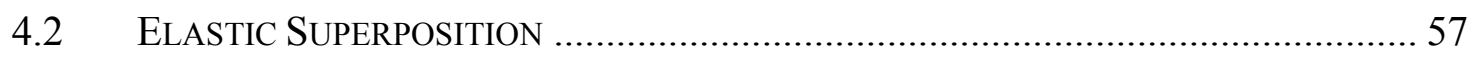

4.2.1 Elastic Superposition Theory ….................................................. 57

4.2.2 Elastic Superposition Assembly Processes ......................................... 59

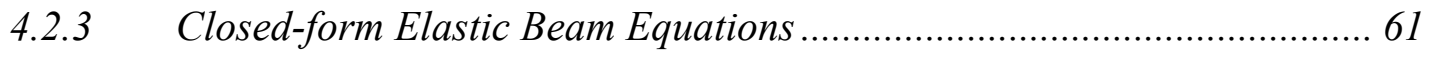

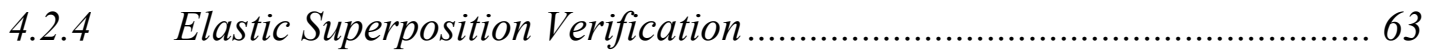

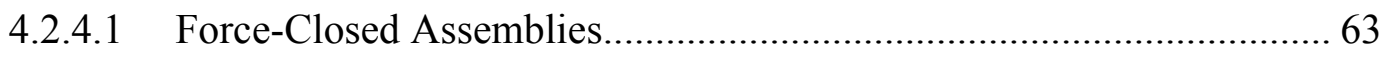

4.2.4.2 Force- and Moment-Closed Assemblies ............................................ 65

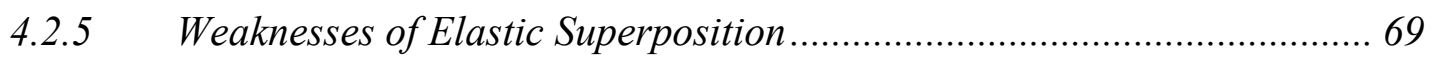

4.3 PieCEWISE-LinEAR ElastiC ANALYSIS...................................................... 69

4.3.1 Piecewise-Linear Elastic Analysis Theory ...................................... 70

4.3.2 Piecewise-Linear Elastic Analysis Assembly Processes........................ 72

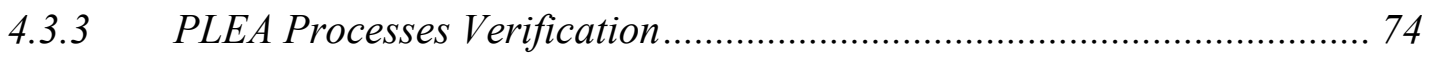

4.3.3.1 Two Beam Assembly, Equal Length, Equal Offset........................... 75

4.3.3.2 Two Beam Assembly, Unequal Length, Equal Offset........................ 77

4.3.3.3 Two Beam Assembly, Equal Length, Unequal Offset........................ 78

4.3.4 Piecewise-Linear Elastic Analysis Comparisons.................................. 79

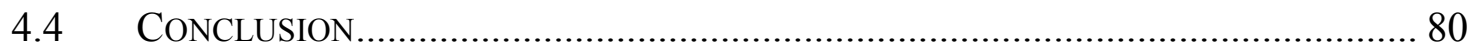

CHAPTER 5: PIECEWISE-LINEAR ELASTIC ASSEMBLY PROCESS .......... 83

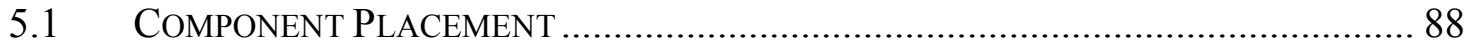

5.1.1 Step 1-Determine Component Misalignments................................... 88

5.1.2 Step 2 - Finite Element Modeling of Compliant Parts ........................... 93 


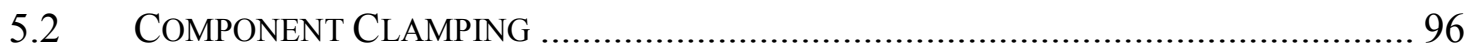

5.2.1 Step 3-Covariance Calculations....................................................... 96

5.2.2 Step 4-Statistical FEA Solution for the Clamping Analysis ................. 100

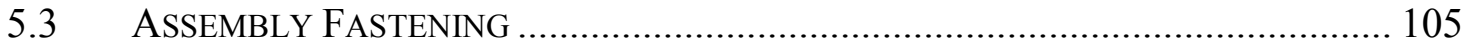

5.3.1 Step 5 - Finite Element Modeling of Clamped Assembly ...................... 105

5.3.2 Step 6-Statistical Springback Force Calculations .............................. 109

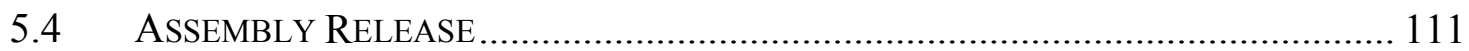

5.4.1 Step 7-Statistical FEA Solution for the Springback Analysis............... 111

5.4.2 Step 8-Overall Statistical Stress Solution Derivation …………........... 114

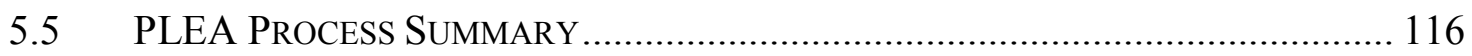

5.6 Alternate ANalysis StePS FOR ComPleX AsSEMBlies.............................. 120

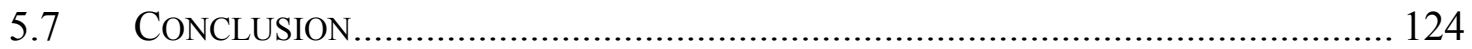

CHAPTER 6: VERIFICATION OF ASSEMBLY ANALYSIS............................... 125

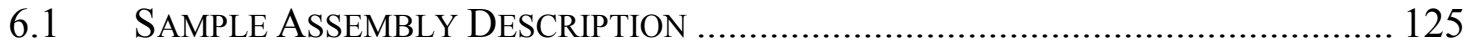

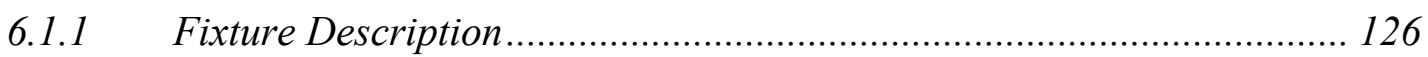

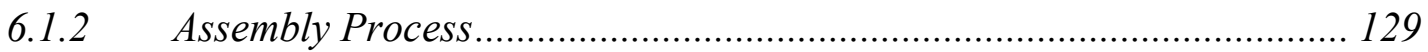

6.1.2.1 Component Placement .................................................................. 129

6.1.2.2 Component Clamping ....................................................................... 131

6.1.2.3 Component Fastening …………………..................................... 132

6.1.2.4 Assembly Release ..................................................................... 133

6.2 ASSEMBLY MEASUREMENT …….......................................................... 134

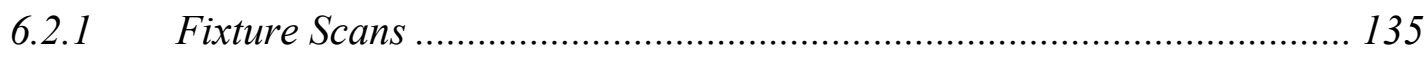

6.2.2 Component Scanning and Modeling .................................................. 135 


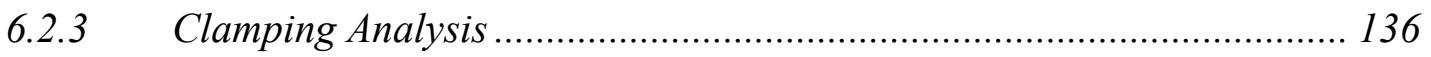

6.2.4 Release and Springback Analysis ................................................... 139

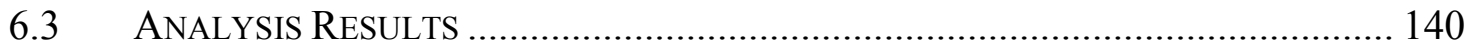

6.3.1 Load Case 1-Near Ideal ................................................................. 142

6.3.2 Load Case 2 - Component Warping .................................................... 146

6.3.3 Load Case 3 - Great Initial Offset.................................................. 150

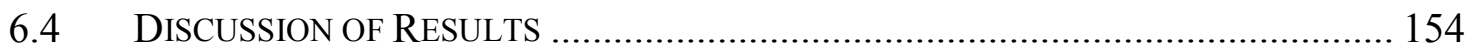

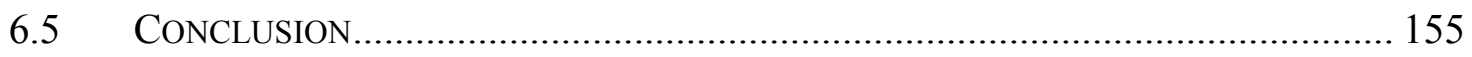

CHAPTER 7: BOEING LEADING EDGE ASSEMBLY ANALYSIS ................. 157

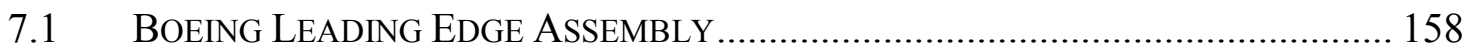

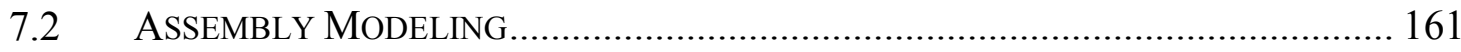

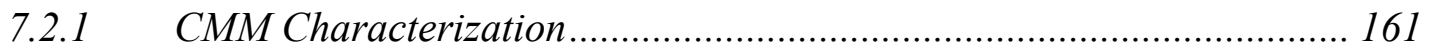

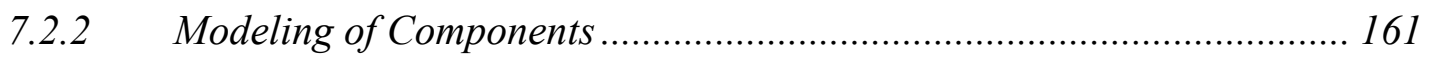

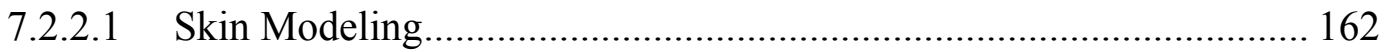

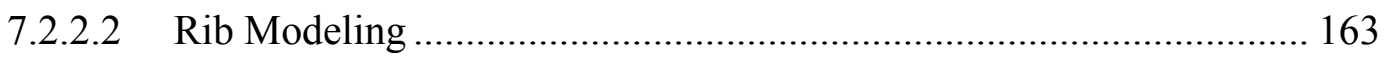

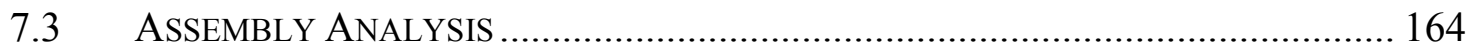

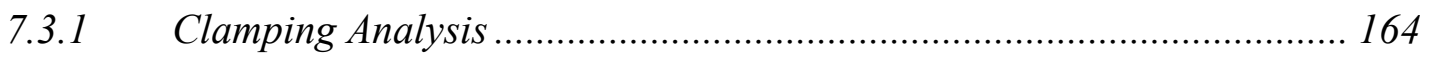

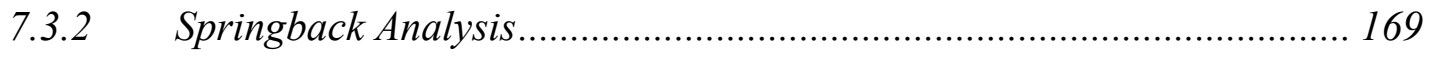

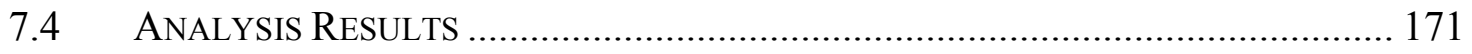

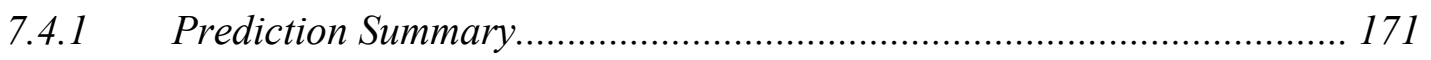

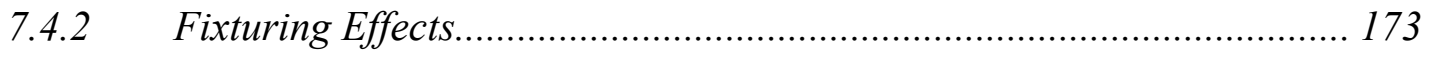

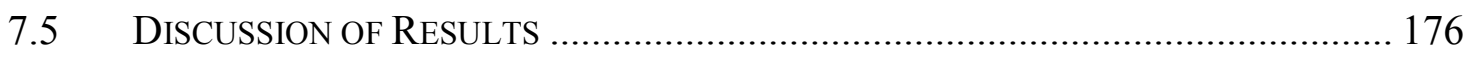

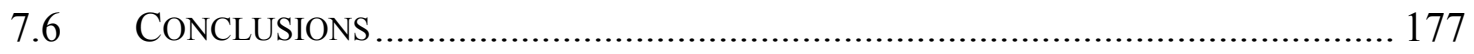




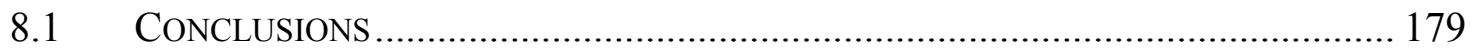

8.1.1 Piecewise-Linear Elastic Analysis Method ....................................... 180

8.1.1.1 The Piecewise-Linear Elastic Analysis Theory …............................ 180

8.1.1.2 PLEA verification ................................................................... 180

8.1.2 Application of PLEA to a real production assembly .......................... 181

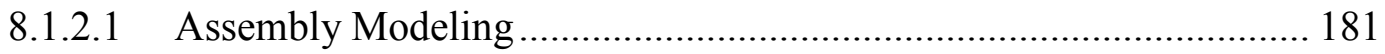

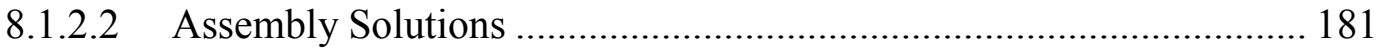

8.1.3 Statistical Tolerance Analysis Flowchart ........................................ 182

8.2 CONTRIBUTIONS ............................................................................... 183

8.3 SugGeSted Future RESEARCH ........................................................... 183

8.3.1 Verify Stochastic Solutions ............................................................ 183

8.3.2 Inclusion of Mid-node Contact and Inelastic Deformations.................. 184

8.3.3 Surface Waviness Characteristic Sensitivity Analysis .......................... 184

8.3.4 Commercial Software Development ................................................ 185

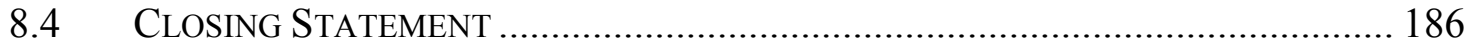

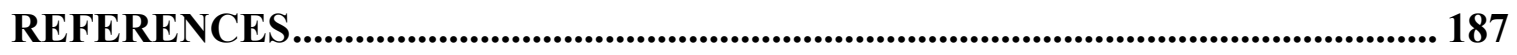

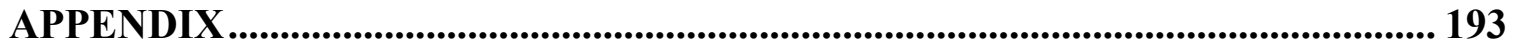

A. Production Sequence of Boeing Leading Edge Assembly ........................ 193

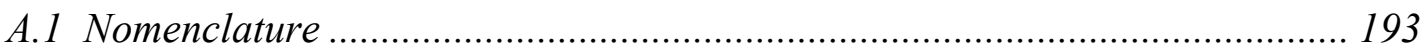

A.2 Assembly Step 1 - Rib Placement .......................................................... 196

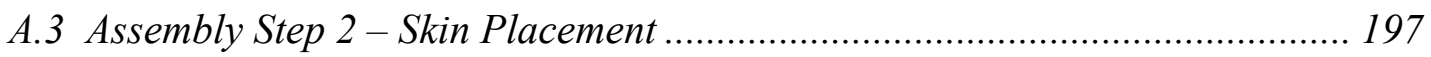

A.4 Assembly Step 3 - Fixture Placement and Clamping................................ 198 


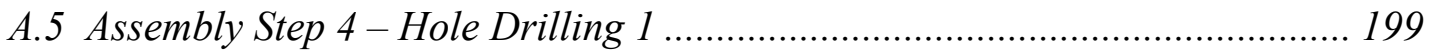

A.6 Assembly Step 5 - Hole Drilling 2 .............................................................. 200

A.7 Assembly Step 6 - Hole Filing............................................................. 201

A.8 Assembly Step 7 - Tapered Shim Installation ........................................... 202

A.9 Assembly Step 8 - Sealant Preparation..................................................... 203

A.10 Assembly Step 9-Component Preparation ........................................... 204

A.11 Assembly Step 10 - Final Hole Drilling................................................... 205

A.12 Assembly Step 11 - Sealant Application ................................................ 206

A.13 Assembly Step 12 - Spring Cleco Pin Insertion ....................................... 207

A.14 Assembly Step 13 - Draw-in Cleco Pin Insertion ..................................... 208

A.15 Assembly Step 14 - Sealant Curing............................................................ 209

A.16 Assembly Step 15 - Rivet Installation ............................................... 210 
xxii 


\section{$\underline{\text { List of Figures }}$}

Figure 1-1: Example of flexible assembly deformation [Mortensen 2002] ....................... 3

Figure 1-2: Flexible assembly deformations with and without the use of a fixture ........... 6

Figure 3-1: Process flow diagram of FASTA process [Mortensen 2002] ……................ 27

Figure 3-2: FASTA step 1 process summary [Mortensen 2002] …………………........ 29

Figure 3-3: Boundary and interior nodes [Tonks 2002] .............................................. 32

Figure 3-4: Equilibrium spring equations [Merkley 1998] ............................................ 34

Figure 3-5: FASTA step 2 process summary [Mortensen 2002] .................................... 36

Figure 3-6: Pictorial illustrations of material and geometric covariances [Tonks 2002] . 38

Figure 3-7: Examples of correlation between $x$ an $y$ [Mortensen 2002]......................... 39

Figure 3-8: Polynomial curve fit of measured data [Tonks 2002] ................................... 42

Figure 3-9: Steps to calculate the autocorrelation function [Tonks 2002]....................... 43

Figure 3-10: The geometric covariance matrix from the autocorrelation function

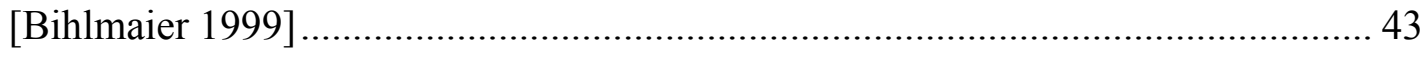

Figure 3-11: Orthogonal polynomial plots [Tonks 2002]............................................. 45

Figure 3-12: Rigid translational and rotational variations [Mortensen 2002] .................. 48

Figure 3-13: Process flow diagram for calculating gap covariance [Mortensen 2002] .... 49

Figure 3-14: Process flow diagram for statistical FEA solution [Mortensen 2002] ......... 52

Figure 4-1: Flexible assembly deformations with and without the use of a fixture ......... 53

Figure 4-2: Two components after the placement stage of an assembly process ............ 55

Figure 4-3: Components A and B are closed (clamped) against the fixture ..................... 56 
Figure 4-4: Components A and B are fastened together. 56

Figure 4-5: The assembly of A and B exhibits springback upon release from the fixture 56

Figure 4-6: A misaligned cantilever beam and fixture 57

Figure 4-7: Resulting shape of the beam when force-closed 58

Figure 4-8: Force and moment acting on end of a beam 59

Figure 4-9: Beams in clamped position 64

Figure 4-10: Springback from nominal position... 64

Figure 4-11: Final positions of assembly. 65

Figure 4-12: Force- and moment-closure of two beams 66

Figure 4-13: Force springback 67

Figure 4-14: Moment springback. 67

Figure 4-15: Force and moment superposition 68

Figure 4-16: Axial and transverse springback components...... 72

Figure 4-17: Final positions of two beam analysis, equal length, equal offset. 76

Figure 4-18: Final positions two beam assembly, unequal length, equal offset ... 77

Figure 4-19: Final Positions of two beam assembly, equal length, unequal offset 78

Figure 4-20: Loading test case 79

Figure 4-21: Ratio of PLEA springback to elastic superposition springback with increasing gap 79

Figure 5-1: PLEA process flow diagram 84

Figure 5-2: Variation contribution example 89

Figure 5-3: Process flow diagram for statistically determining misalignment 92

Figure 5-4: Process flow diagram for FEM of compliant parts 95 
Figure 5-5: Process flow diagram for calculating covariances of the gaps 99

Figure 5-6: Process flow diagram for statistical FEA solution of the clamping analysis 104

Figure 5-7: Model of a fastened assembly using nodal constraints ............................ 106

Figure 5-8: Process flow diagram for FEM of clamped assembly ............................. 108

Figure 5-9: Process flow diagram for calculating the statistical springback forces ....... 111

Figure 5-10: Process flow diagram for statistical FEA solution for the springback analysis

Figure 5-11: Process flow diagram for overall statistical stress solution derivation ...... 116

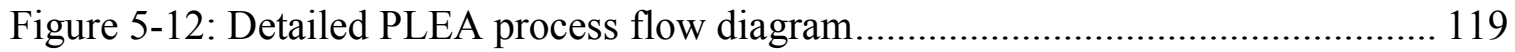

Figure 5-13: Alternate PLEA process flow diagram ............................................ 123

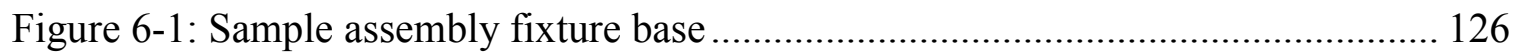

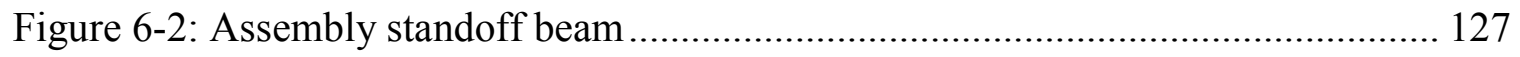

Figure 6-3: Assembly placement and clamping features ...................................... 128

Figure 6-4: Sheet metal components in placement stage ........................................ 128

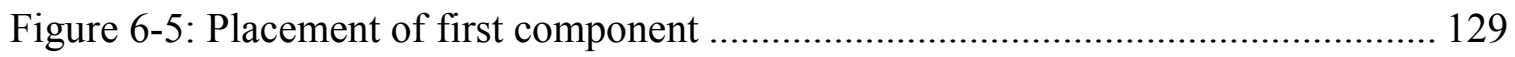

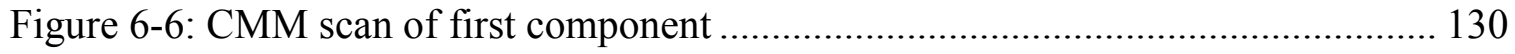

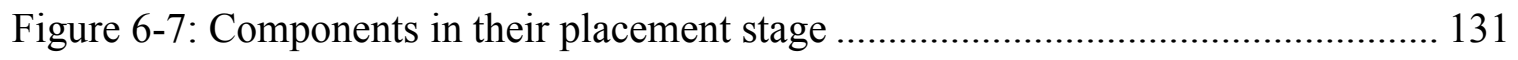

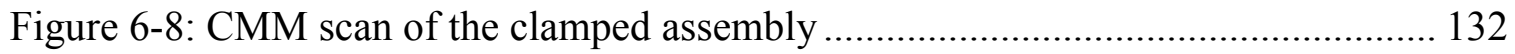

Figure 6-9: Drilling and riveting of the first location ......................................... 133

Figure 6-10: Riveting of exterior locations and completed fastening of assembly ........ 133

Figure 6-11: Release from the clamp and subsequent springback............................. 134

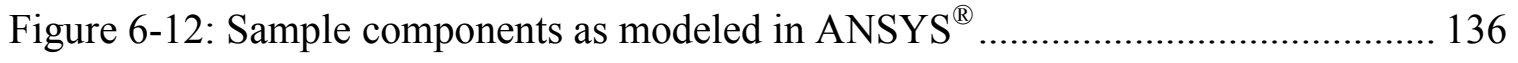

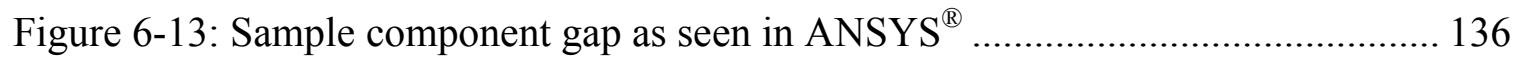


Figure 6-14: Exact constraint on a closure node

Figure 6-15: Over constraint on a closure node

Figure 6-16: Sample cross-section of one plate comparing clamping analysis methods 139

Figure 6-17: Graph section illustration

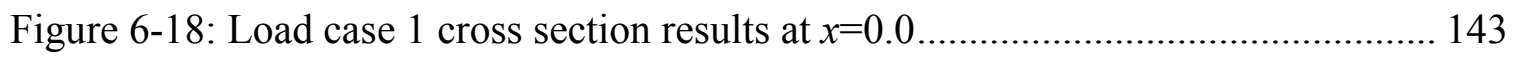

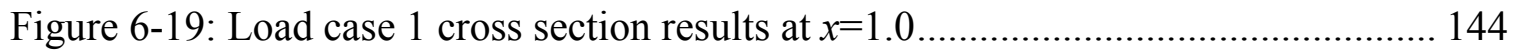

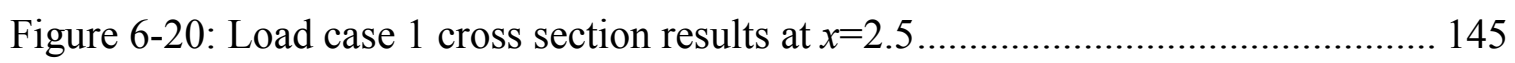

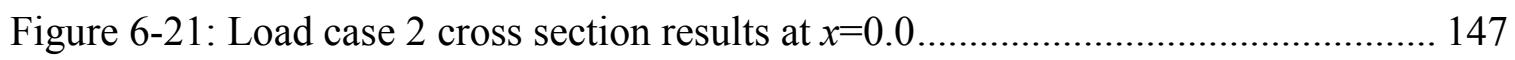

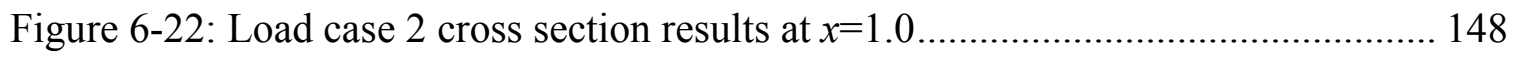

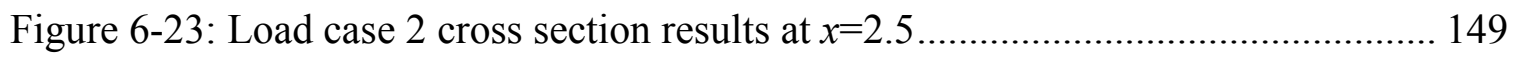

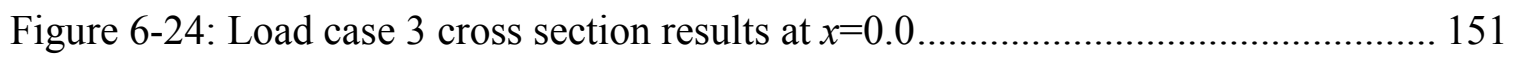

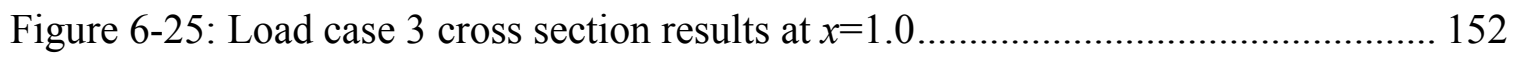

Figure 6-26: Load case 3 cross section results at $x=2.5 \ldots \ldots \ldots \ldots \ldots \ldots \ldots \ldots \ldots \ldots \ldots \ldots \ldots \ldots \ldots \ldots \ldots . . .153$

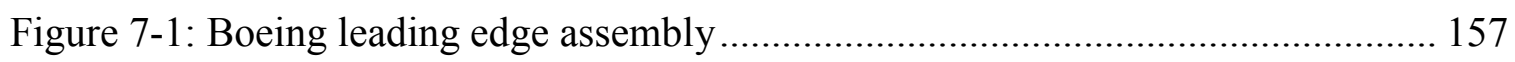

Figure 7-2: Pictures of parts and fixture for leading edge assembly [Mortensen 2002]. 159

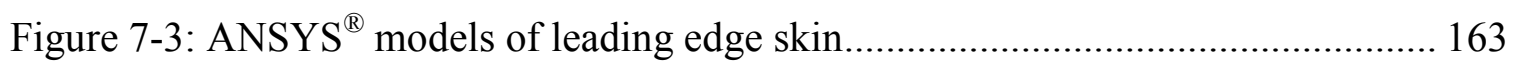

Figure 7-4: ANSYS ${ }^{\circledR}$ models of the leading edge rib stiffeners ....................................... 164

Figure 7-5: Boeing Assembly Cross-section showing Rib, Skin and Fixture Points ..... 166

Figure 7-6: Inaccurate clamping results due to overconstraint ...................................... 167

Figure 7-7: Initial and clamped shape of skin....................................................... 168

Figure 7-8: One cross-section illustrating fixture constraints and skin positions........... 169

Figure 7-9: Ribs and skin modeled for springback analysis ........................................ 170

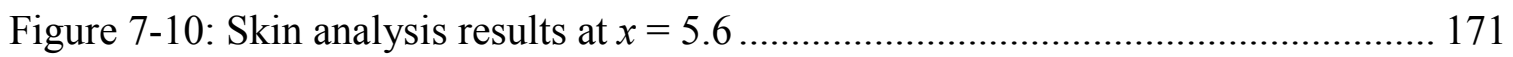


Figure 7-11: Nodes of interest for quantitative comparison ................................... 172

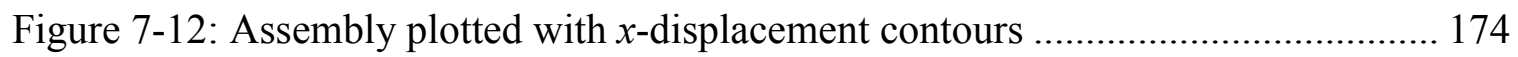

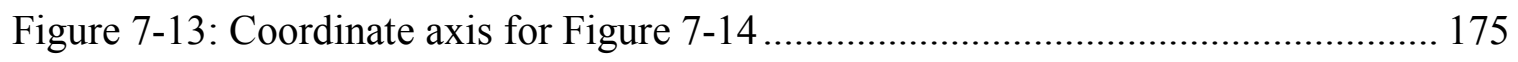

Figure 7-14: “Alligatoring” effect as viewed from assembly top ................................ 175

Figure 7-15: Magnified deformation plot illustrating “alligatoring” of Edge A ........... 176

Figure 8-1: Statistical tolerance analysis method selection ...................................... 182

Figure A-1: The ribs inserted in the backbone fixture ........................................... 196

Figure A-2: Positioning of the skin atop the ribs ................................................... 197

Figure A-3: Clamping of the clamshell fixture over the skin and ribs ......................... 198

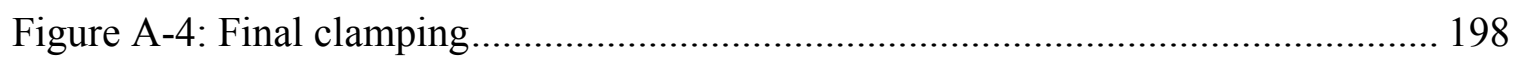

Figure A-5: Drilling and Cleco pin installation ..................................................... 199

Figure A-6: Figure showing the drilling and Cleco pin installation sequence ............... 199

Figure A-7: Figure showing the drilling and Cleco pin installation sequence ............... 200

Figure A-8: Figure showing the drilling and Cleco pin installation sequence, as well as

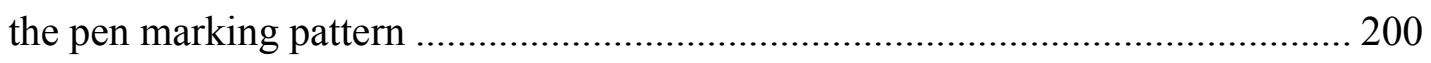

Figure A-9: All component holes are filed to remove burrs ................................... 201

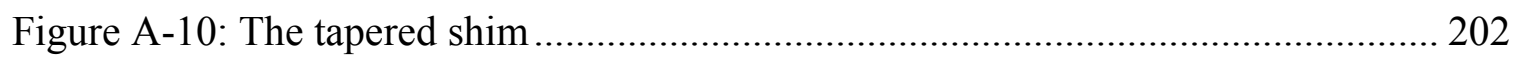

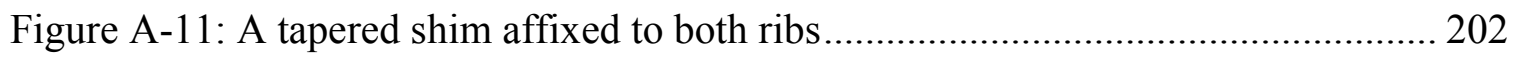

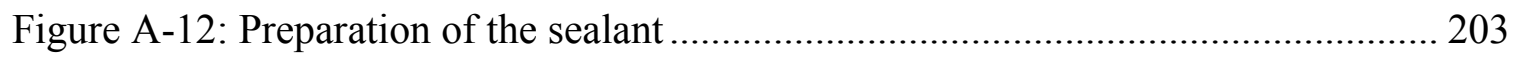

Figure A-13: Cleaning of the parts with denatured alcohol........................................ 204

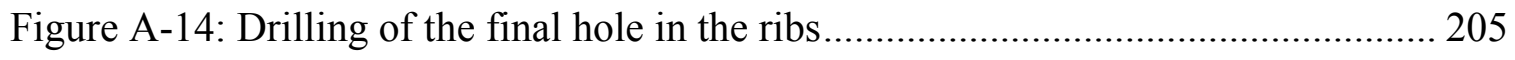

Figure A-15: Application of the sealant to a rib ................................................. 206

Figure A-16: Pre-alignment of rib and skin by operator............................................ 206 


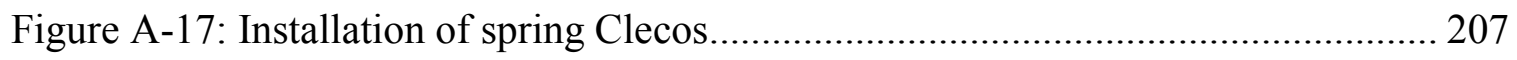

Figure A-18: Pre-alignment of rib and skin by operator......................................... 208

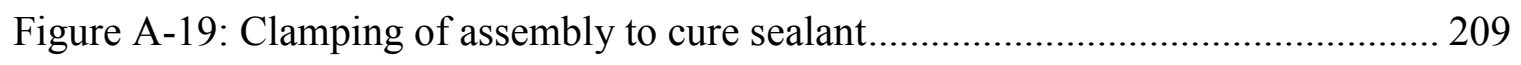

Figure A-20: Procedure to rivet assembly together ............................................. 210

Figure A-21: Assembly showing finished rivet heads ........................................... 211 


\section{List of Tables}

Table 3.1: Geometric covariance estimation method summary [Tonks 2002] ................ 47

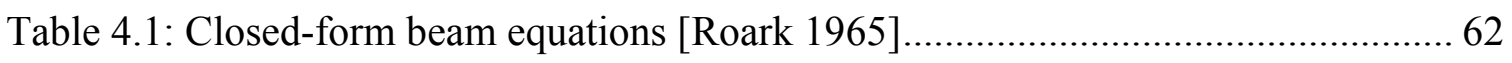

Table 4.2: Beam properties for unequal length, equal offset analysis, force-closure ....... 63

Table 4.3: Beam properties for unequal length, equal offset analysis, force- and moment-

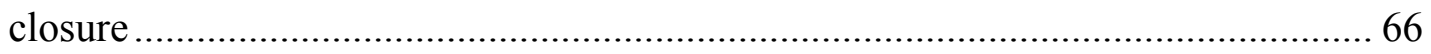

Table 4.4: Beam properties for equal length, equal offset analysis ............................. 76

Table 4.5: Beam properties for unequal length, equal offset analysis ........................... 77

Table 4.6: Beam properties for equal length, unequal offset analysis ........................... 78

Table 5.1: Table of symbols used in the placement and clamping stages ...................... 86

Table 5.2: Table of symbols used in the fastening and release stages ........................... 87

Table 5.3: Summary of steps to determine misalignments ....................................... 91

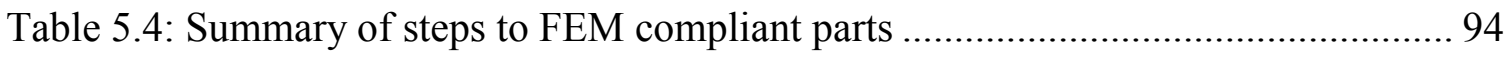

Table 5.5: Summary of steps to calculate covariances of the gaps............................... 98

Table 5.6: Summary of statistical FEA solution for the clamping analysis................... 103

Table 5.7: Summary of steps to FEM the clamped assembly .................................... 107

Table 5.8: Summary of steps to statistical springback force calculations .................... 110

Table 5.9: Summary of statistical FEA solution for the springback analysis ................ 113

Table 5.10: Summary of steps for overall statistical FEA stress solution derivation ..... 115

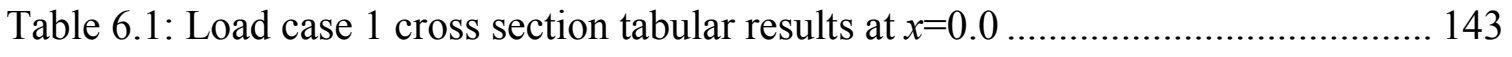

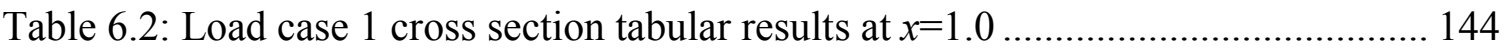




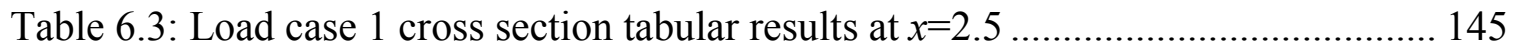

Table 6.4: Load case 2 cross section at $x=0.0$ tabular results .................................. 147

Table 6.5: Load case 2 cross section at $x=1.0$ tabular results ................................. 148

Table 6.6: Load case 2 cross section at $x=2.5$ tabular results .................................. 149

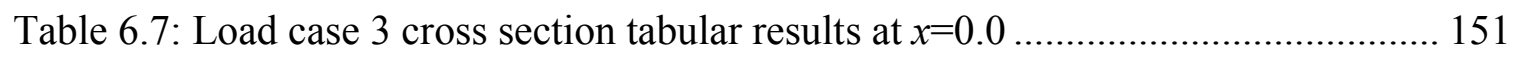

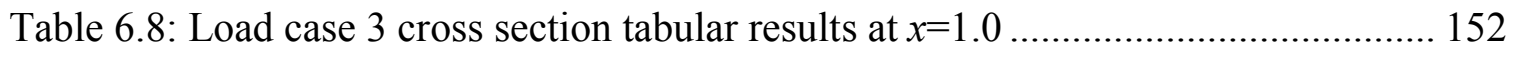

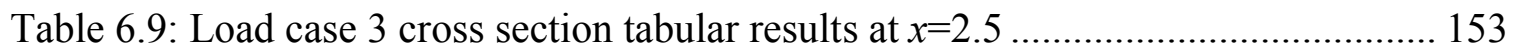

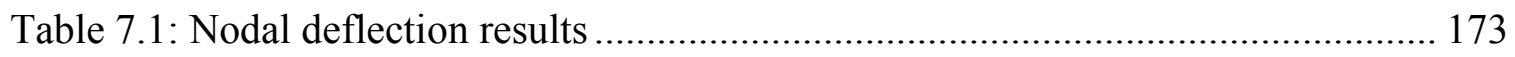

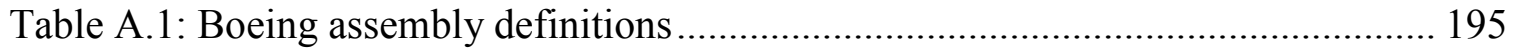




\section{Chapter 1: Thesis Introduction}

\subsection{Introduction}

The purpose of this thesis is to extend methods for analyzing variation in assemblies consisting of flexible components. The research goals include the development of a method for analyzing flexible parts which are assembled by the use of a fixture and a method for applying the analysis using real assembly scans.

\subsubsection{Compliant Assemblies}

Assemblies made of thin, flexible components are ubiquitous in manufacturing today. This is primarily due to the low-cost at which the raw material can be made and the ease at which the component can be formed and assembled. Airplanes, automobiles, machinery guards, air ducts, and consumer goods such as file cabinets, computer panels, and home appliances are examples of assemblies made of flexible components. It is expected that in the future, with an ever-increasing emphasis on manufacturing cost reduction and variation control, design and manufacture of flexible assemblies will continue to increase. 
Individual component tolerances play a large part in the final shape of the assembly. No matter the cost or complexity of the manufacturing process, no part can be made perfectly. Tool and die wear, fixture misalignment, machine drift, material variations, operator variation and countless other factors prevent machines from producing perfect, repeatable parts. For this reason, designers must specify component tolerances and geometric dimensioning, thus establishing envelopes for acceptable parts. In assemblies of many individual components, dimensional variations of the constituent components can lead to tolerance stack-up. In tolerance stack-up, minor variations accumulate from part-to-part and propagate through the assembly, affecting its final form and shape. When the assembly is made up of rigid components, during assembly, the components may interfere with one another or gaps may be left between mating components. The effects of tolerance stack-up are often known immediately; if the rigid parts all "fit", the assembly is usually acceptable.

Flexible members, however, can be deformed or warped to overcome any gaps during assembly. However, such assembly forces cause internal stress in the components. An example of this is shown in Figure 1-1. This internal stress may affect the final form or the expected life of the assembly, or lower the visual aesthetics of the product. The designer of a flexible assembly needs a tool which can predict the final form and stress states of the parts within the assembly, based upon the tolerances specified for the individual parts. With such a tool, the designer strives to specify tolerances tight enough to yield a low-stress assembly having a final form close to that of the intended design; yet loose enough for each part to be easily and economically manufacturable. Thus, 
tolerance allocation is not guesswork, rather, it is a significant engineering task meant to satisfy the requirements of both designers and manufacturers, illustrated in Chase \& Parkinson [1991].

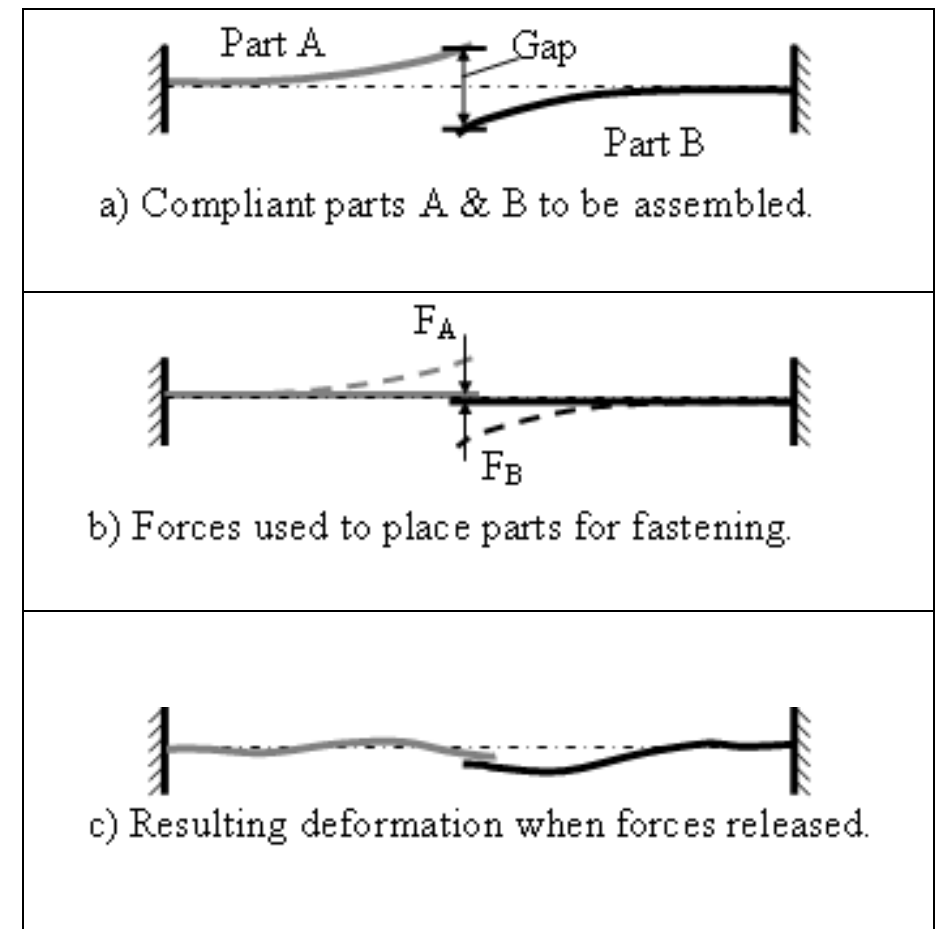

Figure 1-1: Example of flexible assembly deformation [Mortensen 2002]

\subsubsection{FASTA Introduction}

A unique tool is currently being developed at Brigham Young University by students of Dr. Kenneth Chase, with funding from The Boeing Company and the BYU ADCATS consortium. The objective of the BYU flexible assembly research group is to produce a commercial-quality variation analysis tool, integrated with a commercial CAD system and FEA package. This comprehensive system will allow designers to model assemblies 
graphically, to predict the effects of process variations, and to design assemblies which conform to the desired stress and deformation limits.

The system currently being developed is called FASTA, for Flexible-Assembly Statistical Tolerance Analysis. FASTA is used to analyze flexible assemblies for residual stresses and deformations, combining linear elastic finite element analysis (FEA) with traditional first-order statistical tolerance analysis (STA). Using FASTA, the complete stress and deformation state of an assembly are calculated, based upon the statistical tolerances of the individual parts. These states are solved in statistical form for the mean and variance at critical assembly interfaces. The mean solution, based upon the nominal dimensions of the parts, and the variance of the solution, based upon the distribution of the specified tolerances. This analysis will help the designer focus, not only upon the mean, but also the statistical extremes when designing the assembly.

FASTA has advantages over Monte Carlo simulation (MCS), commonly used in industry for variation simulation. For flexible assemblies, MCS involves simulating a number of surfaces (1000 or greater) by sets of random points, each with a unique random profile, assembling these surfaces analytically, calculating by FEA the closure forces for each individual assembly, and plotting a histogram of the results. As with any histogram, the greater the number of simulations, the more the histogram represents the true population. Each MCS is time-consuming, taking significant amounts of time to yield an accurate population. FASTA, however, only requires two FEA calculations (the mean and the variance), and can be accomplished in orders of magnitude less time. 


\subsection{Thesis Objective}

While much groundwork has been laid for implementing FASTA, some common industry assembly practices need to be studied and included in the FASTA method. These needs can be delineated into three objectives this thesis will address. The first objective is to develop an analytical model for fixtured assembly processes, in which the components are assembled while clamped in a rigid fixture. The second objective is to adapt the statistical finite element solution techniques employed with the FASTA method to multi-stepped fixtured processes. The third objective is to systematically apply these methods to an actual fixtured assembly to verify the predicted results.

\subsection{Fixtured Assembly Analysis}

When addressing assembly processes of compliant parts, there are four distinct steps in the process of affixing parts together with the use of a fixture. The first step is to place the parts in the fixture using the native locating features on the fixture. The second step is to clamp the parts rigidly in the fixture to prevent motion in subsequent steps. The third step is to fasten the parts together by welds, rivets, adhesive bonds, threaded fasteners, or any other fastener which will transmit both normal and shear loads. The fourth step is to release the assembly from the fixture. These four steps of assembly are

generally called a PCFR cycle for place, clamp, fasten and release. During production, fixtured inspection processes may also be employed. Four step measurement cycles (PCMR) may be carried out, with the fastening step being replaced by a measurement step. Details may be obtained from Chang [1997] and Chang et al. [1999]. 
Of particular interest to engineers are the assembly forces, displacements, and stresses in the parts which occur at each step, as well as the residual stress, springback, and final form of the assembly. In addition to predicting these results for individual assemblies, product quality goals require estimates of the variation in critical features over the entire population, with predicted yield of assemblies which will meet engineering requirements.

Currently the FASTA method is limited in the way it accounts for the assembly of flexible components. It is currently assumed that the parts are assembled freely, that is, without the use of a fixture to locate the point of fixation of both parts. If a fixture is used, a multi-step analytical process must be employed. The FASTA method cannot account for the clamp, fasten and release operations with a single FEA solution. An illustration of the differences is given in Figure 1-2.

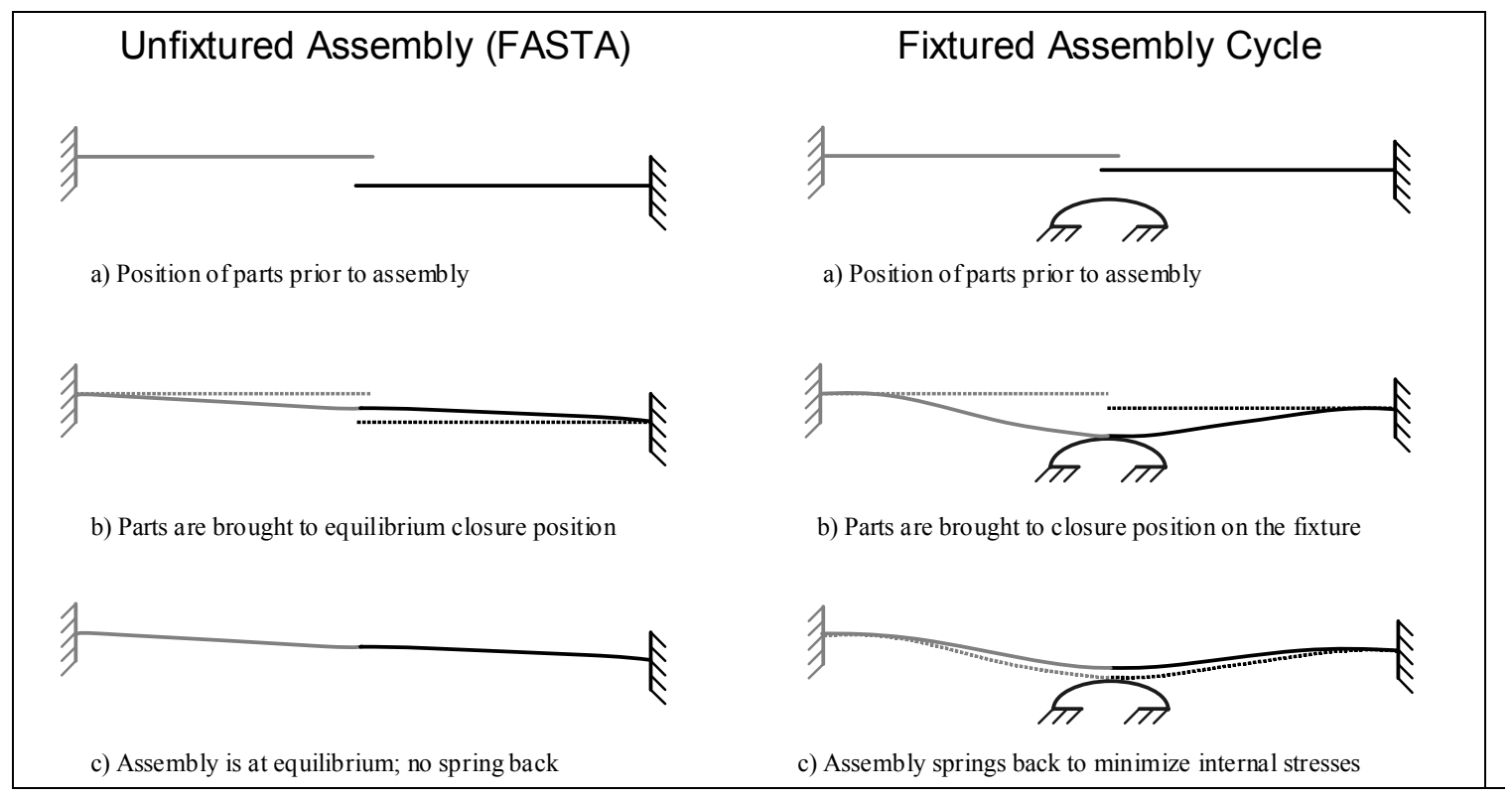

Figure 1-2: Flexible assembly deformations with and without the use of a fixture 
To simulate the effect of a fixture, FEA analysis using the methods of superposition has been investigated. Separate FEA analyses were performed to simulate the steps in the PCFR cycle and combined to predict the spring-back due to a fixtured assembly. The predicted deformation was compared to measured data obtained through experimentation with laboratory specimens. To verify the analysis results, a simple assembly consisting of two plates held rigidly in a fixture has been produced. The PCFR cycle was performed to assemble the two plates. Measurements of the plates were taken before and after assembly. Measurements taken before assembly were used as inputs to the FEA analysis. Measurement data taken from the final assembly were compared to the FEA predicted results.

\subsection{Verification}

To complete the verification procedure, a new analysis method similar to the FASTA method must be applied to the assembly of production parts. Mortensen [2002] has laid the groundwork for implementing the FASTA process on a real assembly obtained from Boeing. A set of parts was pulled from production to be used as a test case. Since his thesis was published, the individual components of the assembly have been scanned on a CMM. In addition, the assembly process was witnessed and the final assembly inspected on a CMM. Using FASTA as a predictive tool, the initial parts were analyzed and assembled analytically by FEA. The simulated assembly was then compared to the final assembly. This serves as the first test of the validity of using FASTA on a complex assembly. Two key modeling concepts were tested: 
1. Modeling the surface variation as specified displacements applied to the ideal geometry, which allows the finite element stiffness matrices to be calculated only once for an entire population of assemblies.

2. Modeling the closure between mating surfaces at a set of discrete contact points (the rivet locations) and ignoring possible contacts occurring between the rivets. In general, if the surface waviness has higher frequency content than the rivet spacing, it will be of much lower amplitude. It is assumed that the closure forces will not be affected appreciably.

Since only one set of production parts were obtained, no verification of the statistical FEA model was possible, but the models for joining imperfect surfaces and estimating the residual stress and deformation is a valuable step in verifying the complete procedure laid out by Mortensen [2002].

\subsection{Scope Delimitations}

The extent of the research done for this thesis was limited in the following ways:

\subsubsection{Statistical Assumptions}

For many manufacturing process variations, it is a reasonable assumption that combinations of independent distributions can be assumed to be normal, due to the central limit theorem. Therefore, in the design stage, it is commonly assumed that the 
dimensional variations exhibit normality, with most of the parts between the specification limits located at $\pm 3 \sigma$. This is a powerful assumption. It follows that the gap to be closed can be assumed to be normally distributed, since it is the result of accumulation of several dimensional and form variations. Furthermore, because closure force and stress are linear functions of the gap distance, we can assume they also exhibit normality.

\subsubsection{FEA Assembly Analysis}

It is assumed that the components are clamped rigidly in a fixture prior to being forceclosed together in the fastening step of the assembly process. With the FASTA method, as with any conventional FEA analysis, all rigid-body motion of individual parts must be removed. Fixturing provides sufficient boundary conditions to achieve this. The tolerance stack-up of the individual component dimensions translates into a specification (mean and variance) of the closure gap, which becomes a displacement input to the FEA analysis. Displacements and deformations are assumed to be small, such that the geometry has not changed sufficient to affect the stiffness matrices.

\subsubsection{Linear Elastic Assumptions of Components and Assemblies}

Because most tolerance bands are small compared to the overall part dimension, we can assume the relationship between closure force and component displacement is linear. Material and geometric nonlinearities in any analysis step are assumed to be negligible. It is further assumed during closure, as the closure nodes are brought in contact with each

other, no mid-node contact occurs. This assumption is required to utilize linear elastic 
FEA and first order STA. Additionally, it is assumed that process nonlinearities, such as welding, localized distortion or plastic yielding would not be considered during the fastening process.

\subsubsection{Access to Appropriate Software Packages and Equipment}

For the initial solution of the mean force, FASTA requires the stiffness matrix for the compliant parts. While this can be calculated by hand, for complex parts with multiple closure points this calculation becomes very cumbersome. For this reason, it is assumed the user has access to a FEA package which can solve for and output the stiffness matrix [K] for any given part. Additionally, Bihlmaier [1999] indicates that once the closure force is calculated, the global constitutive $[D]$ and kinematic $[B]$ matrices are needed to produce a stress solution which must also be obtained from the FEA package. All FEA software packages solve the same sets of basic equations; very little distinguishes them functionally behind their Graphical User Interface or modeling capabilities. It is assumed the user has access to and can output the needed information from a FEA package. ANSYS $^{\circledR}$ version 6.0 was used throughout this thesis. Additional operations, such as matrix inversion and multiplication, or multivariate solving must be performed externally on the products of FEA solutions, and an appropriate mathematic program should be used to make these computations easier. Mathcad ${ }^{\circledR}$ 2001i Professional and Microsoft ${ }^{\circledR}$ Excel 2002 were used for such purposes.

For the verification of FASTA on complex assemblies, a Coordinate Measuring Machine (CMM) was used to determine exact surface points in 3-dimensions. The measurements 
were performed on a Brown \& Sharpe ${ }^{\circledR}$ CMM, using PC-DMIS ${ }^{\circledR}$ version 3.0 software, to follow part contours and output the scan points. The points were then converted to surfaces by the modeling capabilities of ANSYS ${ }^{\circledR}$.

\subsubsection{Normal Closure Forces and Moments}

When the flexible parts are clamped against a fixture, it is assumed the loads and moments are concentrated at the closure nodes and directed exactly towards the fixture. It is further assumed that only the theoretical minimum force and moment required for closure will be considered and modeled.

\subsubsection{Mean Verification}

FASTA can be verified on production assemblies, but to do so statistically would call for many production assemblies. Because only one assembly was obtained from Boeing, no verification of the statistical FEA model was possible in this thesis.

\subsection{Thesis Summary}

This chapter has presented the objective and described the research tasks for this thesis. In order to accomplish this purpose, the thesis has been organized as follows:

Chapter 2 summarizes previous related research, which is further developed throughout this thesis. 
Chapter 3 demonstrates the FASTA method as developed by Merkley and addresses the contributions of Soman, Stout, Bihlmaier, Tonks, and Mortensen.

Chapters 4-5 develop the new analysis method and provide an implementation process map.

Chapter 6 demonstrates the new method in analyzing the assembly of simple components.

Chapter 7 further demonstrates the new method on a complex, production-quality assembly.

Chapter 8 presents conclusions and significant findings, as well as recommendations for future research.

It is hoped that industry will benefit from this tool through lowering production costs and reducing assembly variation. It is further hoped that the contributions of this thesis will make compliant assembly analysis tools more robust and useful to flexible assembly designers. 


\section{Chapter 2: $\quad$ Background Research}

Variation analysis of flexible assemblies brings together four areas of study:

- Rigid Body Tolerance Analysis

- Finite Element Analysis

- Spectral Analysis

- Flexible Assembly Processes

Previous research at BYU has progressed toward integrating all four areas into a comprehensive system for tolerance analysis of flexible assemblies as an interactive, graphic, CAD application.

This chapter summarizes the research this thesis builds upon. Additionally, this chapter summarizes the research accomplished at Brigham Young University toward the development of the FASTA method.

\subsection{Rigid Body Tolerance Analysis}

Two tasks commonly performed by engineering designers and manufacturing personnel to quantify assembly variation are tolerance analysis and tolerance synthesis. Tolerance analysis estimates the accumulation of individual dimensional variations to ensure the 
resulting assembly variation will be within specification limits and predicts the percentage of rejects. Tolerance synthesis assigns individual component tolerances from the given assembly specification limits and quality level. Both are powerful analysis tools. Engineers desire tight tolerances to insure fit, functionality and performance. Manufacturers desire loose tolerances to lower production costs, to compensate for operator skill or machinery precision, and to reduce non-value added activities like scrap and rework. Tolerance analysis and synthesis are important design functions, which allow both the engineer and manufacturer to satisfy their competing requirements.

A survey of common rigid-body tolerance analysis practices was written by Chase \& Parkinson [1991]. They show three main methods in which to conduct tolerance analysis: worst-case, statistical, and sampled methods. The worst-case tolerance analysis method assumes all the components are simultaneously at their worst specification limits. This very conservative method assures even at statistically improbable events, all assemblies will meet specification limits. This type of analysis takes little computer calculation time, but creates very tight specification limits, which may produce excessive manufacturing costs.

A more liberal approach is statistical tolerance analysis (STA). Root Sum Squares (RSS) tolerance analysis assumes all parts follow a Gaussian, or normal, distribution. This accounts for the low probability of worst-case combinations occurring. RSS Statistical methods take low computer calculation time, but cannot account for non-normal distributions. More advanced STA methods can include non-normal distributions. These 
methods include Hasofer-Lind, the method of moments, and integration. All STA analyses trade-off between tight tolerances and the possibility for rejected assemblies.

Sampling techniques, such as a Monte Carlo simulation, create random sets of parts and assemble them together to simulate the effects of variation. Sampling methods are simpler to apply, but require large numbers of assemblies and consequently, great computation time, to yield an acceptable histogram. However, sampling methods can analyze non-normal and skewed distributions, which leads many designers to use this analysis method over the others, even though most users apply only normal distributions.

Another STA method extends RSS analysis to 2-D and 3-D assemblies. In this method, vector loops are created, representing the dimensional chains in the assembly that control translational and rotational tolerance stack-up. Using first-order Taylor series, the nonlinear equations are linearized, based upon the assumption of small variations from nominal dimensions. Using partial derivatives, sensitivities are found for each vector in the loop and related to variation in each degree of freedom. These sensitivities are cast into matrix form and the overall variation solved for key assembly features. This method, called the Direct Linearization Method (DLM), is illustrated in Mortensen [2002] and is particularly useful in the FASTA method for calculating the mean and variance of the gap at each fastening point due to rigid body dimensional variation.

Monte Carlo simulation can be applied to 2-D and 3-D assemblies, but the nonlinear equations often require an iterative solution for each simulated assembly, which greatly 
increases computation time. Comparative studies with the DLM can be found in Gao et al. [1995], Cvetko et al. [1998], and Glancy and Chase [1999].

\subsection{Finite Element Methods}

Finite Element Analysis (FEA) is a versatile tool which can be used to perform structural, thermal, irrotational fluid flow, and other linear analyses. It is an analysis method which is similar to RSS statistical tolerance analysis. Both methods use linear approximations and both methods are accurate for small perturbations.

FEA is very useful for its ability to analyze complex shapes and assemblies. In FEA, a complex structure is divided into geometrically simple elements. The simple shape of the element allows an approximate polynomial solution to be found for that element. Any continuous function can be approximated by a finite number of piecewise polynomial functions. A solution for stress and deformation throughout a complex structure is obtained by joining elements to approximate the structure and solving simultaneously for the stress and deformation in each element. To increase accuracy in FEA, an analyst may specify smaller and more elements, or create elements with higher order approximations. Because it is the element geometry, not the final geometry, which is important in FEA analysis, this method is very useful in analyzing complex parts.

As the number or complexity of the finite elements increases, the required computation increases dramatically. While the speed at which computers can perform mathematical functions continually increases, still it takes significant amounts of time to create a model 
and complete an accurate complex analysis. Crisfield [1986] introduced the matrix condensation method to reduce the analysis to only those nodes in the stiffness matrix important for the analysis, without a reduction in accuracy. A complex structure can be divided into a few "superelements" with greatly reduced degrees of freedom. Such a reduction makes the stiffness matrix smaller and the analysis easier.

This method is well suited for analyzing assemblies of multiple parts, since each part can be represented as a superelement. The first step of such an analysis would be to divide each part into conventional finite elements. After that, the stiffness matrix of each part is reduced. Loads and boundary conditions are applied to the assembly of superelements and analyzed to determine the loads and displacements at the adjoining surfaces. After analyzing the assembly, each individual part is analyzed with its full matrix using the loads and boundary conditions from the previous solution.

\subsection{Flexible Assembly Variation Analysis}

While tolerance analysis has been studied for some time, including part compliance in tolerance analysis of assemblies is a relatively new field of study. As of yet, no standard procedure for performing this type of analysis exists. This section highlights the significant research in this field.

University of Michigan professor Dr. Jack Hu was the first to study tolerance analysis of deformable assemblies. Liu and $\mathrm{Hu}$ [1995a], considered the PCFR assembly and spot welding of two cantilever beams, they realized shear forces are vital to accurate spring- 
back prediction. They implemented an offset beam element, rather than a regular beam element, to account for the shear force in the weld nugget. They verified this method by Monte Carlo simulation. In Liu and Hu [1995b], they looked at the spot-welding sequence of two misaligned beams. They showed how, depending on the sequence, weld joints may absorb or magnify assembly variation. By comparing the expected assembly variations obtained analytically, they found the optimum welding sequence for their test assembly. In Liu and $\mathrm{Hu}$ [1997], they introduced another method using influence coefficients, or the ratio of the part stiffness to the assembly stiffness, to derive the sensitivity matrices. They showed that by using influence coefficients, a typical analysis can be significantly shortened compared to Monte Carlo Simulation.

At the Massachusetts Institute of Technology, Chang [1996] and Chang and Gossard [1997] modeled flexible parts during PCFR cycles. They developed analytical models showing the effects of misalignment within the place, clamp, and fasten stage. These models were limited to a butt-joint configuration spot weld on flanged parts, and only included the expected nominal misalignment using mathematic methods.

Sellem and Rivière [1998] combined the methods of Liu and Hu [1997] and Chang and Gossard [1997], into a comprehensive method. Their main purpose was to avoid Monte Carlo Simulation and its associated computer costs. Using PCFR cycles to account for all variation, they developed influence coefficients to estimate the variability of each part. Sellem et al. [1999a and 1999b] validated this method on an assembly of four complex parts. They were able to compare the means and ranges of the simulation with the real 
assembly. They provided a sensitivity analysis method to identify the key assembly characteristics.

Hochmuth et al. [1998] suggested that part compliance must be included for optimizing tolerances of assemblies. Using rigid parts, they postulated that FEM could be used to include the part compliance by the creation of the part stiffness matrix. They also postulated that covariance matrices can be applied to estimate the statistical distribution. They do not provide examples of the application of these theories.

Recent efforts in Dr. Hu's research have been aimed at modeling the variation of compliant multi-station assemblies. Lee et al. [2000] used sensitivity matrices to evaluate the robustness of a design in a multi-stage assembly. They evaluated single, series, and parallel assemblies using the sensitivity matrices as a step towards predicting the most robust assembly sequence at the part design stage. Camelio et al. [2001] introduced a method of predicting variation propagation in multi-station assemblies. They created different matrices to account for part compliance, the point-to-point sensitivity on the part, and the relationship between positioning schemes in sequential steps. They developed this method for both fixtured and unfixtured assemblies. Dahlström et al. [2002] analyzed a simple 2-part assembly using ABAQUS ${ }^{\circledR}$ and MATLAB ${ }^{\circledR}$. They showed how a design matrix, the Orthogonal Latin Hypercube in their case, can be used to determine high-order interactions. They introduced contact characteristics into the model for more accurate predictions, but at the expense of longer computation times and possible non-convergence. 
Min Hu et al. [2001] presented a method similar to Chang [1996] for predicting assembly deformations. They used a numerical solution involving ANSYS ${ }^{\circledR}$ FEM to combine part and tooling variations with elastic and contact properties to estimate the assembly characteristics. They validated the method by two assembly examples. Their solutions displayed variation, but they did not provide a method for variation prediction.

Camelio et al. [2002] developed a method for fixture design. Using the methods of Liu and $\mathrm{Hu}$ [1997], they determined the expected assembly variation due to all sources of variation. By summing all assembly variation and minimizing a function involving this sum, they found the optimal fixture locations for a simple assembly. Camelio [2002] and Camelio et al. [2003] introduced new advances in their analysis method, called CAVA for compliant assembly variation analysis. They demonstrated the need for geometric covariance when determining the variance, particularly when multiple fastening points are used or 3-dimensional analysis is desired. They developed a new method using assembly variation vectors and eigenvalues to compute the geometric covariance in much less time than with influence coefficients.

\subsection{Flexible Assembly Statistical Tolerance Analysis}

BYU doctoral student Karl Merkley [1996, 1998] was the first to propose the FASTA method as currently performed at BYU. By linearizing the elastic contact between joined parts, Merkley developed a method for predicting the mean and variance of assembly forces and deformations. This method uses only two finite element solutions, one for the mean configuration, and one to solve for the expected variance. The variance is obtained 
from a statistical covariance solution. Merkley also introduced the need for surface continuity conditions to represent surface errors as a continuous surface, not independent nodes. He developed a method using random Bèzier curves to characterize the surface variation in terms of a covariance function called the geometric covariance. The geometric covariance was found to be very dependent on the wavelength of the surface waviness.

Bihlmaier [1999] developed a method to estimate the geometric covariance by using the autocorrelation function from spectral analysis. This method transforms the entire frequency spectrum of the surface waviness into a single covariance matrix. By transforming the random surfaces to the frequency domain, the autospectrum can be obtained. The autospectrum is then transformed back into the spatial domain to create the autocorrelation function, which is then used to simulate the geometric covariance. This method is known as the spectral analysis method and is useful when surface variations are sinusoidal.

Stout [2000] also developed a method to estimate the geometric covariance by using a polynomial curve fit. This method is known as the polynomial method. The polynomial method was easier to use than the Bèzier curve method, but neither it nor the Bèzier curve method could account for sinusoidal variations.

Soman [1999] created a set of 6 real parts to determine experimentally the geometric covariance. Up to this time, parts with simulated surface variation were used to verify the 
analysis methods. Using the spectral analysis method developed by Bihlmaier [1999], he characterized the parts to find an autocorrelation function to define the set. Through experimentation, he discovered the spectral analysis method cannot analyze parts with sinusoidal variations where the wavelength is greater than the part length. He determined that long wavelengths must be separated from shorter wavelengths in order to successfully apply the spectral analysis method. He suggested that the polynomial method should be applied to the long surface wavelengths and spectral analysis applied to the short and medium wavelengths.

Tonks [2002] developed three new, robust methods for estimating the geometric covariance. Using a set of sinusoids to estimate the error, he developed a robust method which could analyze parts with sinusoidal variations. Using orthogonal polynomial series like the Legendre polynomials, he was able to develop a method which could analyze parts with polynomial variations and large-wavelength sinusoidal variations. This method he used to analyze the parts created by Soman [1999] with good results. Combining these two methods with weighting coefficients, he created a hybrid method which could analyze parts with any type of variation. He tested these methods on two simulated assembly conditions, one with part flexibility and one with part rigidity.

Mortensen [2002] synthesized and laid out the FASTA method into easy-to-follow flowcharts. Also, using rigid-body tolerance analysis, he included macro sources of variation, such as part translation, rotation, and surface effects, into the gap covariance equation. 
With the inclusion of geometric covariance into the FASTA method, an accurate prediction of the force and deformation ranges is possible. However, specific information on the magnitude and wavelength of variation components which describe surface waviness is required. This requires knowledge of the production process as well as intensive data-gathering and computation. 


\section{Chapter 3: Analysis Using the FASTA Method}

This chapter outlines the methods to perform FASTA analysis, which have been developed at Brigham Young University. This outline relies upon the previous work of Mortensen [2002], who organized and diagrammed the method.

\subsection{FASTA Basics}

As was mentioned previously, FASTA produces both a mean and a statistical solution. To produce both solutions, the following two equations are solved.
Mean:
$\left\{\mu_{F}\right\}=[\mathbf{K}]\left\{\mu_{\delta}\right\}$
Eq. 3-1
Covariance: $\quad\left[\Sigma_{F}\right]=[\mathbf{K}]\left[\Sigma_{\delta}\right][\mathbf{K}]^{T}$
Eq. 3-2

The performance of FASTA can be divided into four distinct steps. The first three steps consist of finding values for the FASTA equations, and the fourth step is to solve the above equations. Each step will be discussed in detail in the following sections:

1. Determine Misalignment using Statistical Tolerance Analysis - Finds $\left\{\mu_{\delta}\right\},\left\{\sigma_{\delta}\right\}$

2. Finite Element Modeling of Compliant Parts - Calculates [K]

3. Covariance Calculation - Estimates $\left[\Sigma_{\delta}\right]$

a. Initial Methods

i. Bèzier Curve Method 
ii. Frequency Spectrum Method

iii. Polynomial Method

b. Robust Methods

i. Polynomial Series Methods

ii. Sinusoidal Method

iii. Hybrid Method

4. Statistical Finite Element Analysis - Solves for $\left\{\mu_{F}\right\}$ and $\left[\Sigma_{F}\right]$
a. Primary Solution
b. Part Solutions and Post-Processing

A flowchart developed by Mortensen [2002] is presented in Figure 3-1 to illustrate the method. Additionally, it is important to note that FASTA can be used both in the design stage as well as with a set of real production parts to be assembled. FASTA can be used as a variance prediction tool in the design stage. More powerfully, it can be used to predict the error in a single assembly or a population. Both uses will be addressed. 


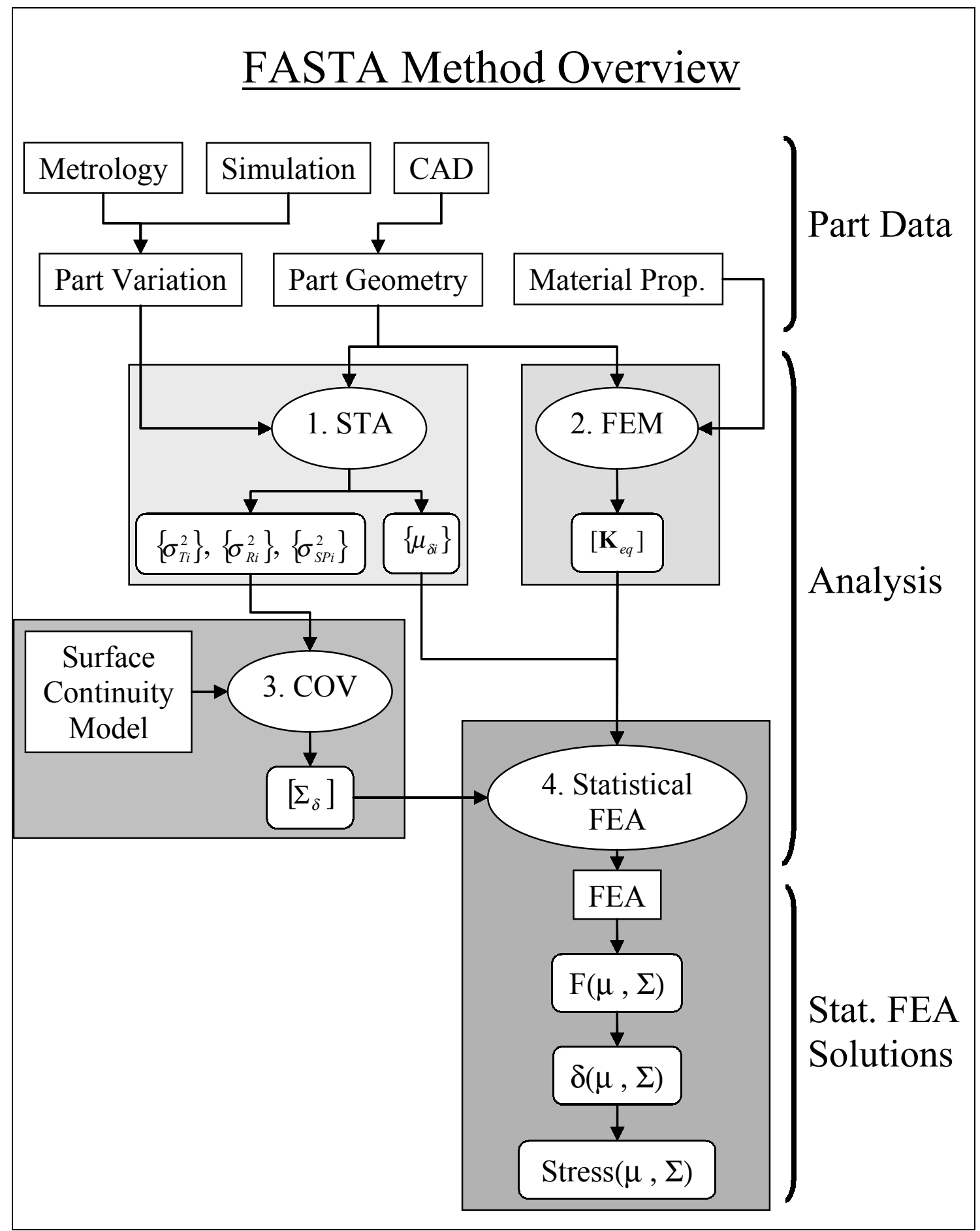

Figure 3-1: Process flow diagram of FASTA process [Mortensen 2002] 


\subsection{Determine Misalignment}

The first step in the FASTA method is to determine the misalignment between mating surfaces prior to closure. The misalignment typically a gap between two surfaces, with a set of closure points where fasteners or joining processes are to be applied. The gap must be characterized statistically for input to FEA analysis.

\subsubsection{Real Assemblies}

For assemblies of real production parts, datum surfaces are identified for each part, and key dimensions measured. The closure points of mating surfaces must be located prior to closing. From these scans of the gap between the points of closure, both the mean $\left\{\mu_{\delta}\right\}$ and the covariance $\left[\Sigma_{\delta}\right]$ may be obtained directly, thus characterizing the gap statistically. As with all statistical methods, a large sample population better describes the entire population.

\subsubsection{Simulated Assemblies}

For simulated assemblies, misalignment is determined by modeling the assembly, with the parts at their nominal positions and assuming the parts are rigid. The DLM is used to predict the mean gap and the variance about the mean due to the accumulation of dimensional variations. Random part variations arise as a result of random process variations. These variations in dimensions, angles, feature size, position, orientation, etc. may be included. If process data are available, the mean and standard deviation of each 
variation source may be used. If none are available, they may be estimated from similar production parts. If no similar production parts are available, specified tolerances may be assumed to represent the process variations. The entire tolerance band is commonly assumed to be six standard deviations.

Additionally, the propagation of variation due to rigid body effects such as part rotation $\left\{\sigma_{R}^{2}\right\}$, part translation $\left\{\sigma_{T}^{2}\right\}$, and the surface finish callouts $\left\{\sigma_{S P}^{2}\right\}$ must be evaluated for a further step. A detailed example is presented in Figure 3-2 by Mortensen [2002].

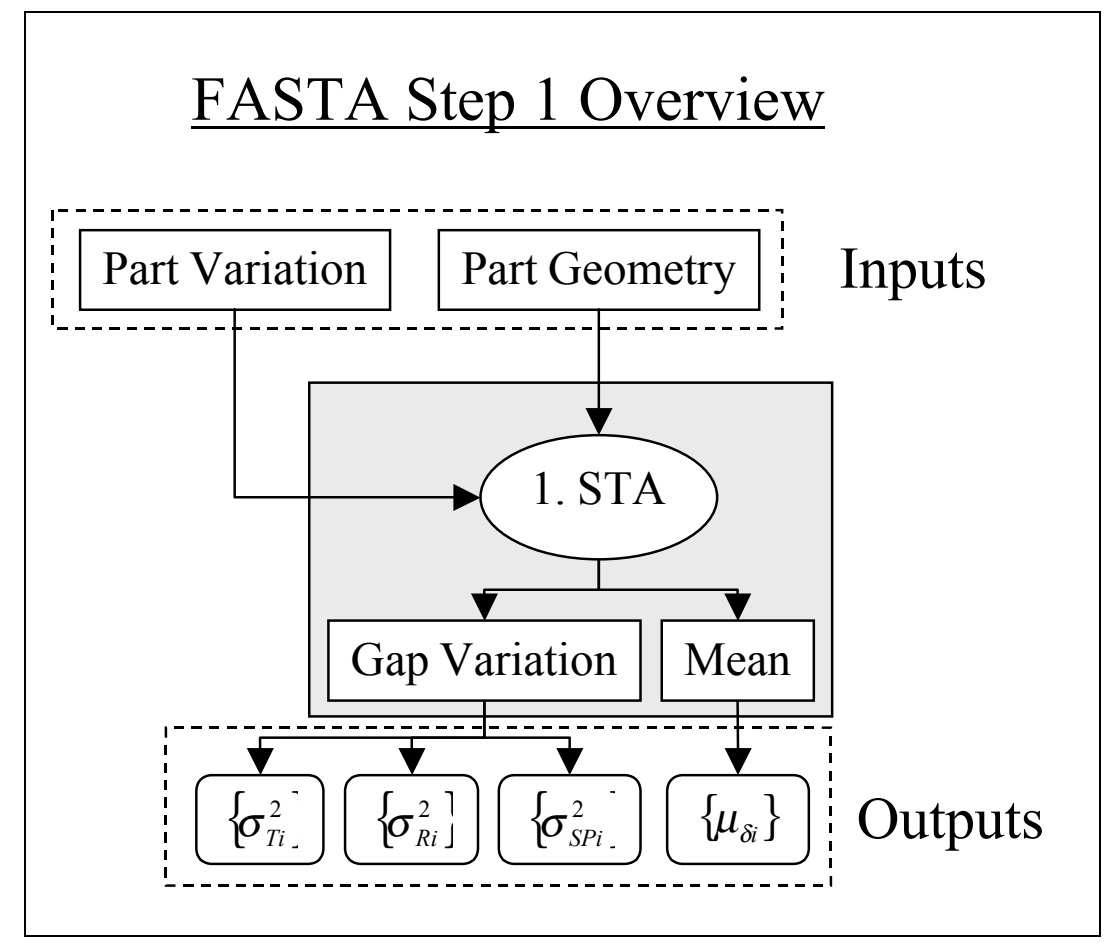

Figure 3-2: FASTA step 1 process summary [Mortensen 2002] 


\subsection{Finite Element Modeling of Compliant Parts}

The Finite Element Method is commonly applied to complex geometrical structures which have no closed-form solution. The structure is divided into smaller pieces for which a closed-form solution may be estimated. These pieces, or elements, have points in common with neighboring elements where force equilibrium can be assumed. The common points are called nodes. Inherent in the finite element method is the need to estimate the stiffness between neighboring nodes, thereby estimating the force between nodes resulting from nodal displacements. Much as a linear spring, flexible parts have some stiffness $(k)$, and the force $(F)$ equals the stiffness times the displacement $(\delta)$ from some natural position. The basic force equation for any linear spring is:

$$
F=k \delta
$$

A flexible part with multiple nodes behaves similar to a system of linear springs.

However, rather than a single value to describe the stiffness of the part, the stiffness needs to be expressed in matrix form, thereby incorporating stiffness interactions of one node with all other nodes in the part. The force and deflection are expressed as vectors and the stiffness as a matrix in the following form:

$$
\{F\}=[\mathbf{K}]\{\delta\}
$$

Eq. 3-4

In Equation 3-4, [K] is the stiffness matrix of the part, and $\{F\}$ is a vector of forces required to deflect the part a certain distance $\{\delta\}$. For the FASTA method, $\{\delta\}$ is defined as the mean gap distance $\left\{\mu_{\delta}\right\}$, and $\{F\}$ becomes the mean closure force, $\left\{\mu_{F}\right\}$, for each closure node. The common form of the force-deflection equation for the FASTA method is given in Equation 3-5. 


$$
\left\{\mu_{F}\right\}=[\mathbf{K}]\left\{\mu_{\delta}\right\}
$$

FEA methods for deriving the overall stiffness matrix $[\mathbf{K}]$ from individual element stiffnesses are well documented and will not be reviewed here.

\subsubsection{Substructuring}

In FEA analysis, a node is only influenced by its nearest neighbors. This means any stiffness matrix for a complex part is large, but sparsely populated. Crisfield [1986] introduced a technique which can reduce the size of a stiffness matrix to those nodes important to the analysis without any loss in accuracy. The resulting reduced stiffness matrix is often called a super-element matrix and the technique of creating a superelement matrix is called substructuring.

To reduce a stiffness matrix by substructuring, one must distinguish between internal nodes and boundary nodes. Boundary nodes are those nodes vital to the analysis for one of three reasons:

1. Boundary nodes define points where other parts will be connected

2. Boundary nodes are points at which loads or moments are applied

3. Boundary nodes are points which have nodal constraints

Internal nodes are those nodes which do not fall into one of these categories. While the information they impart is important, it is not necessary for an overall stiffness matrix. Figure 3-3 shows the distinction between boundary and internal nodes on a cantilever plate with an applied end load. 


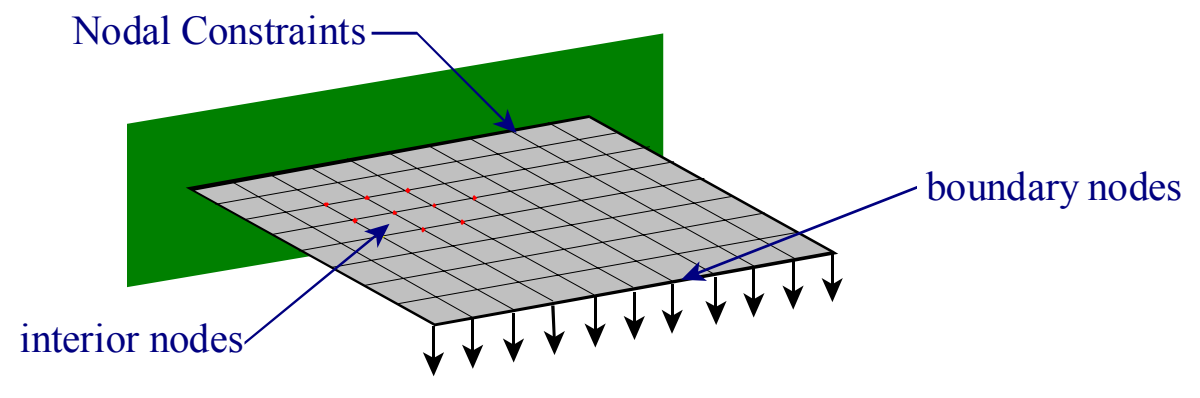

Figure 3-3: Boundary and interior nodes [Tonks 2002]

Once the boundary nodes are identified, the part stiffness matrix can be arranged such that the boundary forces and displacements are separated from the internal forces and displacements. The stiffness equation can then be written:

$$
\left\{\begin{array}{c}
F_{b} \\
\hdashline F_{i}
\end{array}\right\}=\left[\begin{array}{c:c}
\mathbf{K}_{b b} & \mathbf{K}_{b i} \\
\hdashline \mathbf{K}_{i b} & \mathbf{K}_{i i}
\end{array}\right]\left\{\begin{array}{c}
\delta_{b} \\
-\delta_{i}
\end{array}\right\}
$$

Where $\left(F_{b}\right)$ and $\left(\delta_{b}\right)$ are the forces and displacements applied to the boundary nodes and $\left(F_{i}\right)$ and $\left(\delta_{i}\right)$ are the forces and displacements applied to the internal nodes. Because there are no external forces or displacements applied to the internal nodes, each value of $\left(F_{i}\right)$ is 0 . The resultant stiffness matrix is called a partitioned matrix. The submatrices describe the stiffness between boundary nodes $\left(\mathbf{K}_{b b}\right)$, stiffness between internal nodes $\left(\mathbf{K}_{i i}\right)$, and coupled stiffness interactions $\left(\mathbf{K}_{i b}, \mathbf{K}_{b i}\right)$. When solved, Equation 3-6 produces two distinct systems of linear stiffness equations:

$$
\begin{gathered}
\left\{F_{b}\right\}=\left[\mathbf{K}_{b b}\right]\left\{\boldsymbol{\delta}_{b}\right\}+\left[\mathbf{K}_{b i}\right]\left\{\boldsymbol{\delta}_{i}\right\} \\
\left\{F_{i}\right\}=\left[\mathbf{K}_{i b}\right]\left\{\boldsymbol{\delta}_{b}\right\}+\left[\mathbf{K}_{i i}\right]\left\{\boldsymbol{\delta}_{i}\right\}=0
\end{gathered}
$$

Because we defined internal nodes as those without prescribed nodal displacements or applied external forces, Equation 3-8 can be reduced to: 


$$
\left\{\boldsymbol{\delta}_{i}\right\}=-\left[\mathbf{K}_{i i}\right]^{-1}\left[\mathbf{K}_{i b}\right]\left\{\boldsymbol{\delta}_{b}\right\}
$$

Eq. 3-9

Substituting Equation 3-9 into Equation 3-7 yields the force at the boundary nodes alone:

$$
\left\{F_{b}\right\}=\left[\mathbf{K}_{b b}\right]\left\{\boldsymbol{\delta}_{b}\right\}-\left[\mathbf{K}_{b i}\right]\left[\mathbf{K}_{i i}\right]^{-1}\left[\mathbf{K}_{i b}\right]\left\{\boldsymbol{\delta}_{b}\right\}
$$

Eq. 3-10

which can be simplified:

$$
\left\{F_{b}\right\}=\left(\left[\mathbf{K}_{b b}\right]-\left[\mathbf{K}_{b i}\right]\left[\mathbf{K}_{i i}\right]^{-1}\left[\mathbf{K}_{i b}\right]\right)\left\{\boldsymbol{\delta}_{b}\right\}
$$

The reduced stiffness matrix from Equation 3-11 is:

$$
\left[\mathbf{K}_{r e d}\right]=\left[\mathbf{K}_{b b}\right]-\left[\mathbf{K}_{b i}\right]\left[\mathbf{K}_{i i}\right]^{-1}\left[\mathbf{K}_{i b}\right]
$$

Equation 3-11 relates the boundary forces to the boundary displacements alone. It has far fewer degrees of freedom, because all the interior nodes have been eliminated. However, the stiffness for forces applied on the boundary nodes is the same as the unreduced system of equations. Using the reduced stiffness matrix greatly reduces array storage and computation of the closure forces. It is also well-suited for assemblies, since each part may be modeled as a single superelement.

ANSYS $^{\circledR}$ has built-in functions for creating reduced stiffness matrices. It is therefore desirable for the superelement matrix to be created directly by the finite element package. Mortensen [2002] illustrates how to create a superelement matrix in ANSYS $^{\circledR}$.

\subsubsection{Equivalent Stiffness Matrix}

Merkley [1998] has shown how these super-element matrices are useful when two plates are attached at equilibrium. It is possible to use the stiffness matrix of each part to create 
a combined stiffness matrix representing the stiffness of both parts at equilibrium.

Consider the joining of two springs when force equilibrium is achieved in Figure 3-4:

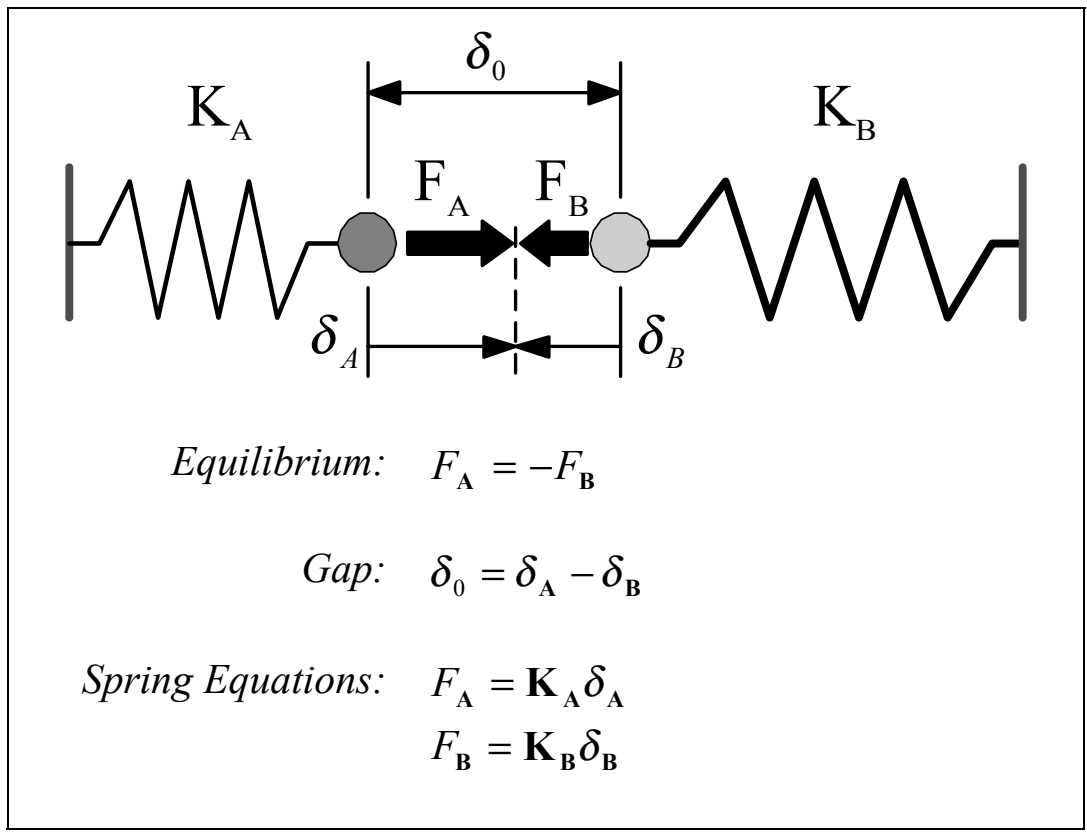

Figure 3-4: Equilibrium spring equations [Merkley 1998]

By combining the above equations as in Merkley [1998], the individual displacements, $\delta_{\mathrm{A}}$ and $\delta_{\mathrm{B}}$, can be solved in terms of the overall displacement, $\delta_{0}$, and the individual spring stiffness values, $\mathbf{K}_{\mathbf{A}}$ and $\mathbf{K}_{\mathbf{B}}$.

$$
\begin{aligned}
& \delta_{\mathrm{A}}=\frac{\mathbf{K}_{\mathrm{B}}}{\mathbf{K}_{\mathrm{A}}+\mathbf{K}_{\mathrm{B}}} \delta_{0} \\
& \delta_{\mathrm{B}}=-\frac{\mathbf{K}_{\mathrm{A}}}{\mathbf{K}_{\mathrm{A}}+\mathbf{K}_{\mathrm{B}}} \delta_{0}
\end{aligned}
$$

This is useful because it is often not known what the individual displacements are, but part stiffness values and the overall gap can be readily determined. Using force equilibrium and substituting Equations 3-13 and 3-14 for the individual spring displacements, the force-displacement equation becomes: 


$$
F_{\mathbf{A}}=-F_{\mathbf{B}}=\frac{\mathbf{K}_{\mathbf{A}} \mathbf{K}_{\mathbf{B}}}{\mathbf{K}_{\mathbf{A}}+\mathbf{K}_{\mathbf{B}}} \delta_{0}
$$

Where $\frac{\mathbf{K}_{\mathbf{A}} \mathbf{K}_{\mathbf{B}}}{\mathbf{K}_{\mathbf{A}}+\mathbf{K}_{\mathbf{B}}}$ is the equivalent stiffness of the springs when joined.

Thus, the stiffness values of each spring act in series with each other when attached at equilibrium. Therefore, if one of the springs has infinite stiffness (a rigid body), the equivalent stiffness approaches the stiffness of the compliant part, and the compliant spring displacement approaches the full range of the overall displacement.

For multiple closure points, Equation 3-15 may be expressed in matrix form:

$$
\left\{F_{\mathbf{A}}\right\}=-\left\{F_{\mathbf{B}}\right\}=\left(\left[\mathbf{K}_{\mathbf{A}}\right]\left(\left[\mathbf{K}_{\mathbf{A}}\right]+\left[\mathbf{K}_{\mathbf{B}}\right]\right)^{-1}\left[\mathbf{K}_{\mathbf{B}}\right]\right)\left\{\delta_{0}\right\} \quad \text { Eq. 3-16 }
$$

The expression of both stiffness matrices becomes the equivalent stiffness matrix:

$$
\left[\mathbf{K}_{e q}\right]=\left[\mathbf{K}_{\mathbf{A}}\right]\left(\left[\mathbf{K}_{\mathbf{A}}\right]+\left[\mathbf{K}_{\mathbf{B}}\right]\right)^{-1}\left[\mathbf{K}_{\mathbf{B}}\right]
$$

The equivalent stiffness matrix can then be used in the FASTA solution, since it describes equilibrium conditions at the closure of an assembly gap. And, as shown in Equation 3-16, we can use the equivalent stiffness matrix, once the mean gap and the gap variance are known, to evaluate the mean closure force and closure force covariance.

To summarize, the steps for modeling a compliant assembly in the FASTA method requires the part geometry and the part material properties. In a FEA program such as ANSYS $^{\circledR}$ each part is meshed into nodes and elements and the resulting part stiffness matrix is found. The boundary nodes are identified and stiffness matrix is substructured to reduce complexity. After each compliant part has a substructured stiffness matrix, these matrices are combined into the equivalent stiffness matrix. The equivalent stiffness 
matrix is the stiffness of the entire assembly acting in resistance to closure of the gap.

This process is illustrated by Figure 3-5.

\section{FASTA Step 2: FEM of Compliant Parts}

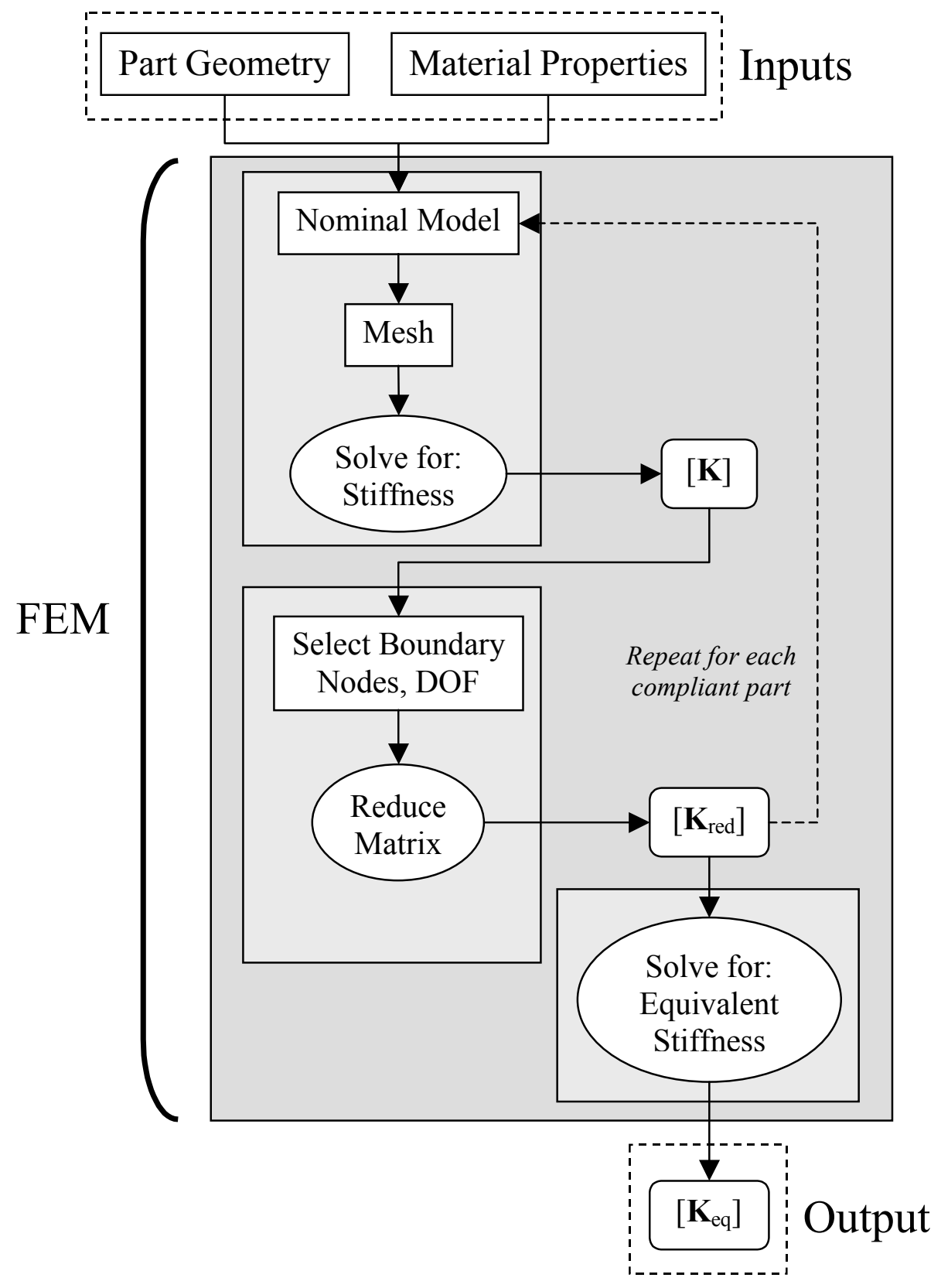

Figure 3-5: FASTA step 2 process summary [Mortensen 2002] 


\subsection{Covariance Calculation}

Covariance is a measure of interdependence between random variables. Merkley [1996] introduced the need for two types of covariance when analyzing flexible assemblies. The material covariance is the most familiar covariance in analysis and is defined by the stiffness matrix $[\mathbf{K}]$. The material covariance relates elastic coupling of neighboring nodes, and is a function of the part dimensions and material properties. Geometric

covariance, $\left[\Sigma_{G}\right]$, accounts for the surface continuity of a part, something which the material covariance does not. The geometric covariance allows a random surface to be analyzed, rather than a set of independent random points, as demonstrated in Mortensen [2002]. The geometric covariance is dependent upon the material, machine, and manufacturing process. The application of geometric covariance is yet another advantage of analyzing with FASTA rather than MCS. MCS typically assumes complete independence between neighboring nodes of a surface. The difference between the two covariance matrices is illustrated in Figure 3-6. 


\begin{tabular}{|c|c|}
\hline \multicolumn{4}{|c|}{ Material Covariance: } \\
\hline $\begin{array}{c}\text { Elastic independence between } \\
\text { neighboring nodes }\end{array}$ \\
{$[\mathbf{K}]=\left[\begin{array}{cccc}K_{11} & 0 & \ldots & 0 \\
0 & K_{22} & & 0 \\
\vdots & & \ddots & \vdots \\
0 & 0 & \ldots & K_{i i}\end{array}\right]$} \\
{$[\mathbf{K}]$ is populated on diagonal only } \\
No material covariance
\end{tabular}

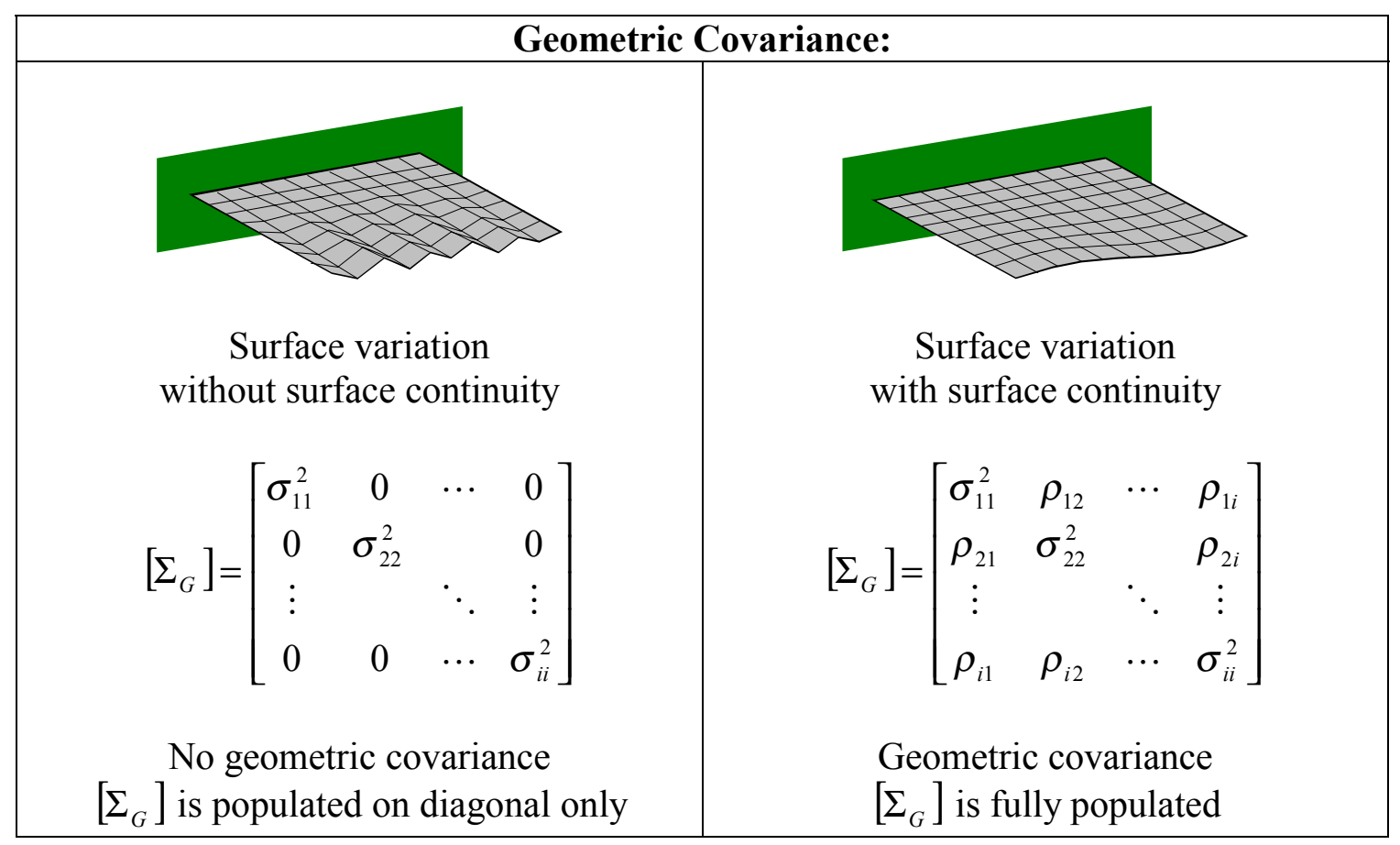

Figure 3-6: Pictorial illustrations of material and geometric covariances [Tonks 2002] 


\subsubsection{Covariance Introduction}

Points on a continuous surface interact. Merkley [1998] showed that in order to accurately model dependent statistical properties, the interdependent relationship must be included in the equation. Such a relationship of interdependence is called the covariance, and is defined by Equation 3-18.

$$
c_{i j}=\sum_{n=1}^{N} \frac{\left[X_{n i}-\bar{I} \rrbracket\left\lfloor X_{n j}-\bar{J} \rrbracket\right.\right.}{N-1}
$$

$\mathrm{X}_{\mathrm{ni}}$ is the nth value of $\mathrm{X}_{\mathrm{i}}$ and $\bar{I}$ is the mean of $\mathrm{X}_{\mathrm{i}}$, and $\mathrm{X}_{\mathrm{nj}}$ is the nth value of $\mathrm{X}_{\mathrm{j}}$ and $\bar{J}$ is the mean of $X_{j}$. The solution of this equation determines the covariance of $i$ and $j$. A positive covariance indicates the second variable increases as the first increases, and a negative denotes the second variable would decrease as the first variable increases. A covariance of zero denotes complete independence between the variables, whereas a covariance equal to the product of the standard deviations of both values denotes complete dependence, or a linear relationship. The covariance can never exceed the product of the standard deviations of both values. An example of correlation can be seen in Figure 3-7.

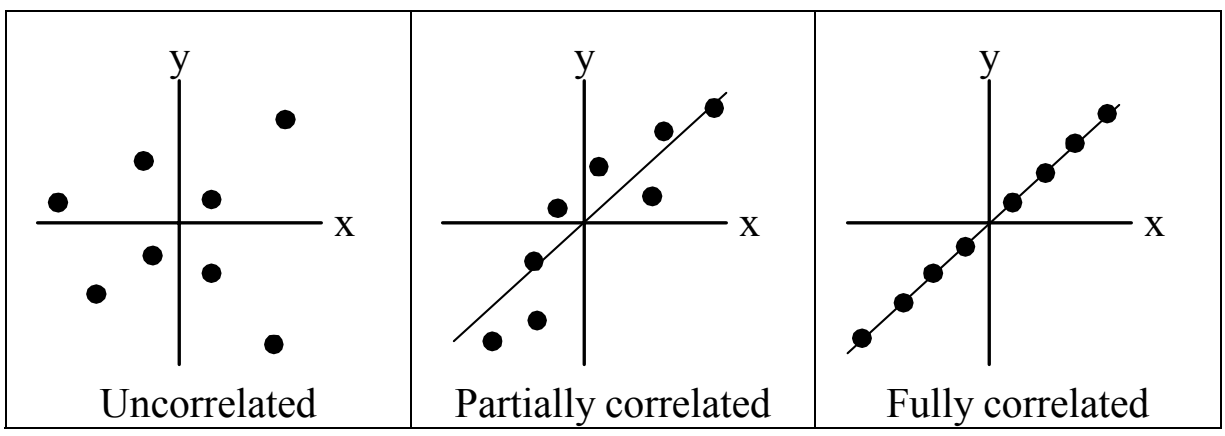

Figure 3-7: Examples of correlation between $x$ an $y$ [Mortensen 2002] 


\subsubsection{Surface Continuity Equations}

Several methods have been developed to determine the geometric covariance, $\left[\Sigma_{G}\right]$.

Most methods rely upon determining the standard deviation of each fastening node from the expected dimensional variations. Once these values are determined, the variance at each node is calculated by squaring each standard deviation. These values are also given in the $\left\{\sigma_{S P}^{2}\right\}$ vector. The resulting matrix is set up according to Equation 3-19, with $\left\{\sigma_{S P}^{2}\right\}$ values populating the diagonal only.

$$
\left[\Sigma_{G}\right]=\left[\begin{array}{cccc}
\sigma_{1}{ }^{2} & 0 & 0 & 0 \\
0 & \sigma_{2}{ }^{2} & 0 & 0 \\
0 & 0 & \ddots & 0 \\
0 & 0 & 0 & \sigma_{n}{ }^{2}
\end{array}\right]
$$

The matrix given by Equation 3-19 assumes complete independence between nodes. Surface continuity equations estimate the surface characteristics of the part. These continuity equations predict nodal covariance and are multiplied to the variances according to Equation 3-20. The solution is the surface covariance matrix for part A.

$$
\left[\Sigma_{G A}\right]=\left[S \rrbracket\left[\Sigma_{G} \llbracket S\right]^{T}\right.
$$

When the surface continuity equations are used, each matrix value can be populated, hence it explains the nodal covariance expected. This is performed for each part, the gap covariance due to translational and rotational rigid-body effects are determined, and all these are added to determine the full gap covariance, given by Equation 3-21.

$$
\left[\Sigma_{\delta}\right]=\left[\Sigma_{G A}\right]+\left[\Sigma_{G B}\right]+\left[\Sigma_{T}\right]+\left[\Sigma_{R}\right]
$$

Eq. 3-21 
Adding the matrices linearly to determine the overall gap covariance assumes complete independence between individual covariances.

\subsubsection{The Bézier Curve Method}

One of the initial methods for estimating $\left[\Sigma_{G}\right]$ was developed by Merkely [1998]. Using random Bézier curves, random surface profiles are created to estimate part variability. Tolerance bands were established for the Bézier curve control points, which controlled the shape of the Bézier curve surface. This initial method, due to the inherent complexity of random Bézier curves, is difficult to implement. For this reason, the Bézier curve method is not used as an estimator of the geometric covariance. Other methods have been developed which are easier to implement.

\subsubsection{The Polynomial Method}

The polynomial method for estimating $\left[\Sigma_{G}\right]$ was developed by Stout [2000]. It uses polynomial curve fits to approximate the actual surface variation. This method is useful because any changes in nodal surface variation will have a diminishing effect as the distance from the node increases. An example of a polynomial curve fit to real data is shown in Figure 3-8. 


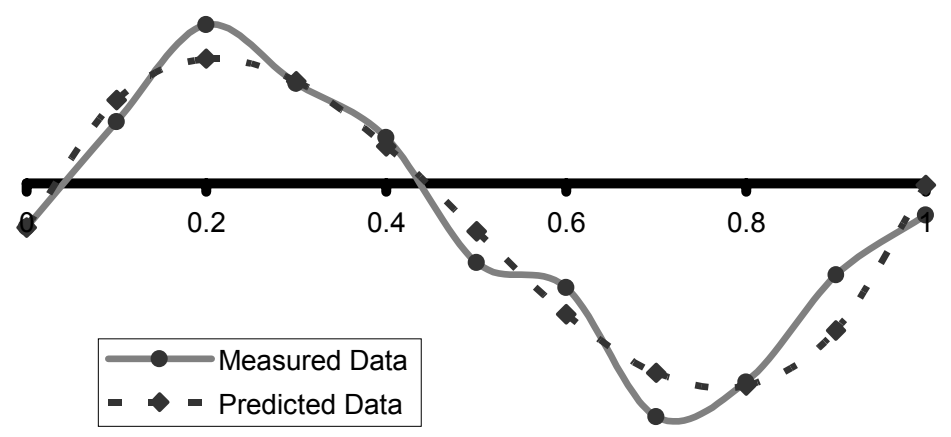

Figure 3-8: Polynomial curve fit of measured data [Tonks 2002]

A sensitivity matrix is created from the polynomial curve fit. Each value in the matrix represents the geometric sensitivity between the predicted value of the curve-fit and the measured data points. To use the polynomial method, the order of polynomial estimating the surface variation must be less than the number of nodes. Because of this, the polynomial method is useful when the part is expected to have polynomial variation. Because the polynomial order must be less than the number of nodes, the polynomial method has difficulty in estimating geometric covariance exhibiting sinusoidal variation with wavelengths shorter than the part length. For this reason, new methods were developed.

\subsubsection{The Frequency Spectrum Method}

Bihlmaier [1999] developed a method using spectral analysis to estimate the surface continuity conditions. Surface scans were made of several parts. The mean part profile was subtracted from each part to eliminate the non-random error. This resulted in only the random variation of the part remaining. By performing a Fast Fourier Transform (FFT), the surface profile is transformed into data in the frequency domain. By 
multiplying each transformed surface by its conjugate, the auto-spectral density function, or autospectrum, is derived. After each part's autospectrum is averaged, the average is inverse Fast Fourier Transformed (IFFT) back to the spatial domain. This result is known as the auto-correlation function. The steps and approximate graphs are seen in Figure 3-9.

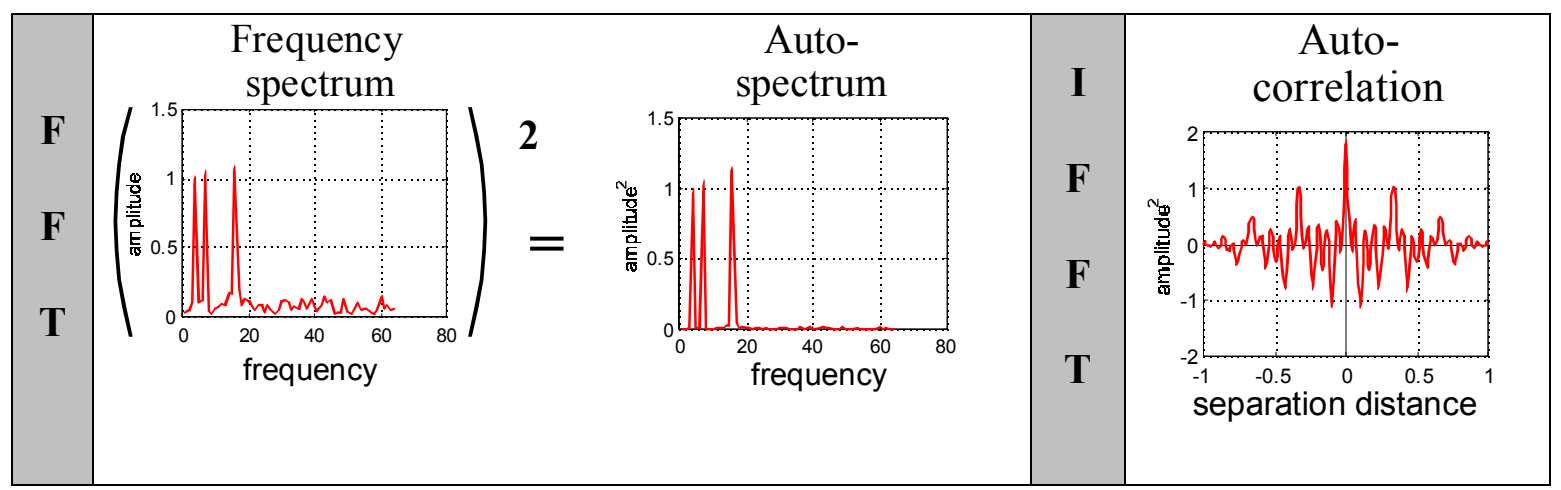

Figure 3-9: Steps to calculate the autocorrelation function [Tonks 2002]

To construct the geometric covariance matrix, the autocorrelation function at zero separation (at the ordinate) must be shifted for each row so the zero separation value falls along the diagonal of the matrix, as shown in Figure 3-10.

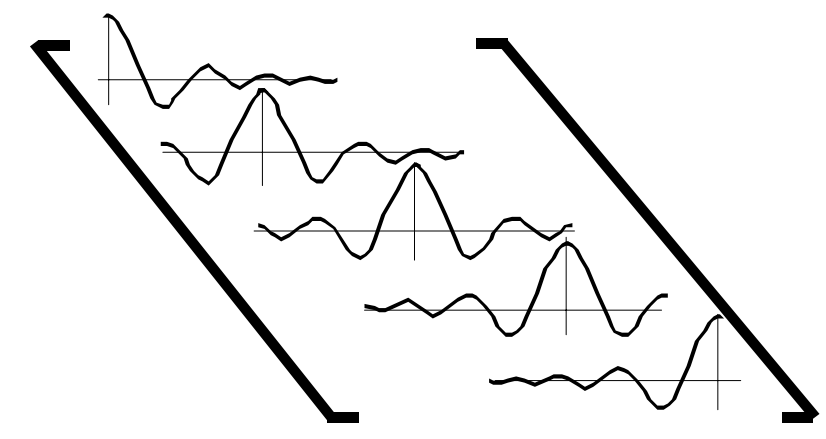

Figure 3-10: The geometric covariance matrix from the autocorrelation function [Bihlmaier 1999] 
The frequency spectrum method has serious limitations. Though it does well predicting the covariance for sinusoidal variations of integer $L / \lambda$ values, it cannot accurately predict the covariance due to non-integer $\mathrm{L} / \lambda$ values nor can it predict the covariance for sinusoidal variations of $\frac{\mathrm{L}}{\lambda}$ values less than 1 . An additional limitation is its inability to model non-random variation. Additionally, it must be assumed that every node has the same standard deviation. For this reason, Tonks [2002] developed a robust method which could still estimate the geometric covariance of a part exhibiting sinusoidal variation.

\subsubsection{The Sinusoidal Method}

The sinusoidal method was developed by Tonks [2002]. It is similar to the polynomial method, but it represents the error surface as a series of sinusoids, from which the sinusoidal sensitivity matrix $\left[S_{S}\right]$ is derived. The sinusoidal sensitivity matrix is used to calculate the geometric covariance, according to Equation 3-22.

$$
\left[\Sigma_{G P}\right]=\left[S_{S}\right]\left[\Sigma_{S P}\right]\left[S_{S}\right]^{T}
$$

The values for the sinusoidal sensitivity matrix, $\left[S_{S}\right]$, are obtained from the following equation:

$$
\begin{gathered}
S_{S, i j}=\sum_{k=0}^{M-1} \sqrt{\frac{2}{N}} a_{k, \text { norm }} \cos \frac{2 \pi f_{k}}{N}(j-i) \\
\text { where: } \quad a_{k, \text { norm }}=\sqrt{\frac{a_{k}}{\sum_{k=0}^{M-1} a_{k}}}
\end{gathered}
$$


This equation assumes $M$ frequencies and corresponding amplitudes, $a$, can accurately describe the data. Because of the use of sinusoids to estimate the error, the sinusoidal method can model geometric covariance when the variation exhibits both integer and non-integer ${ }^{\mathrm{L}} / \lambda$ variation. The limitation of this method is its inability to model variation where $\mathrm{L} / \lambda$ is less than 1 or exhibits polynomial variation.

\subsubsection{Polynomial Series Methods}

Another accurate method is to use orthogonal sets of polynomials. Tonks [2002] developed methods of using Chebyshev and Legendre polynomials to estimate the geometric covariance. These polynomial sets are normalized over the range of $-1 \leq x_{i} \leq 1$ and are bounded at the ends in the y-direction by 1 and -1 . Graphical examples of these sets of polynomials from 0 to $4^{\text {th }}$ order are seen in Figure 3-11.

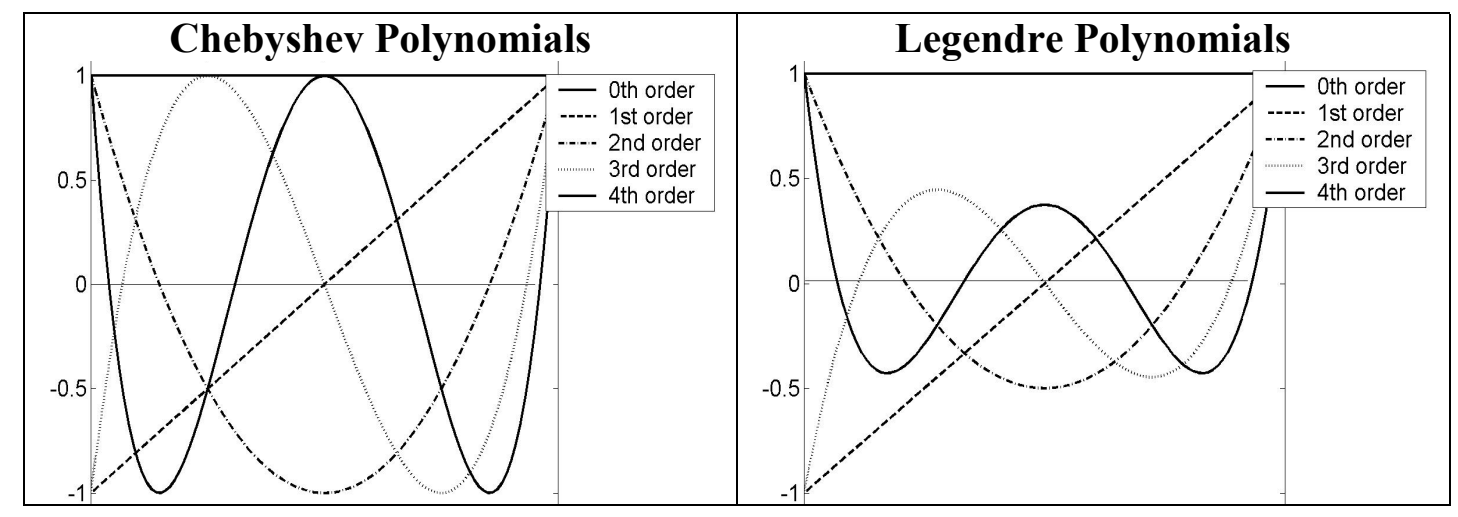

Figure 3-11: Orthogonal polynomial plots [Tonks 2002]

The derivation for the Chebyshev and Legendre polynomial transformation matrices are obtained through similar methods. Because the results obtained from both methods are so similar, only the Legendre method will be considered hereafter. To find the geometric 
covariance transformation matrix, the x-values $\{u\}$ of $N$ terms must be normalized to fit within the bounds of the polynomial series. This is done through Equation 3-24.

$$
x_{i}=\frac{u_{i}-\frac{1}{2}(N+1)}{\frac{1}{2}(N-1)}
$$

Once the $\mathrm{x}$-values are normalized, each polynomial order up to $M$ is given a weighting coefficient $\{a\}$. The Legendre geometric covariance transformation matrix can be calculated by Equation 3-25.

$$
S_{\mathrm{lg}, i j}=\sum_{l=0}^{M-1} \sqrt{\frac{2 l+1}{N}} a_{k, n o r m} P_{l}\left(x_{j}\right) P_{l}\left(x_{i}\right)
$$

A similar equation is derived for finding the Chebyshev geometric covariance transformation matrix. This geometric covariance transformation matrix can then be used to estimate the geometric covariance according to Equation 3-26.

$$
\left.\left[\Sigma_{G P}\right]=\left[S_{\mathrm{lg}}\right] \Sigma_{S P}\right]\left[S_{\mathrm{lg}}\right]^{T}
$$

Eq. 3-26 


\subsubsection{The Hybrid Method}

A summary of the abilities of all the methods to estimate geometric covariance can be seen in Table 3.1.

Table 3.1: Geometric covariance estimation method summary [Tonks 2002]

\begin{tabular}{|c|c|c|c|c|c|}
\hline & Poly. & S. A. & Sin. & Cheby. & Legen. \\
\hline Geometric Covariance & & & & & \\
\hline Models Polynomial Variation: & Good & Poor & No & Good & Good \\
\hline Models Sinusoidal Variation & & & & & \\
\hline Integer $\lambda /$ values: & No & Good & Good & Poor & Poor \\
\hline Non-integer $\lambda /$ values: & No & Fair & Good & Poor & Poor \\
\hline N v values less then one: & Poor & Poor & Fair & Good & Good \\
\hline
\end{tabular}

A single method which can analyze all cases of surface variation does not exist. For this reason, Tonks [2002] developed a hybrid method which combines the Legendre polynomial series method and the sinusoidal method. This gives the hybrid method the ability to analyze both polynomial and all sinusoidal variations. This is done by calculating both geometric covariances and, with weighting coefficients $a$ and $b$, weighting the covariances respectively based upon the actual surface variation.

$$
\left[\Sigma_{G P_{H}}\right]=\left(\frac{a^{2}}{a^{2}+b^{2}}\right)\left[\Sigma_{G P_{L}}\right]+\left(\frac{b^{2}}{a^{2}+b^{2}}\right)\left[\Sigma_{G P_{S}}\right]
$$




\subsubsection{Rigid Body Contributions}

In addition to the surface variability, Mortensen [2002] developed a method for including the covariant rigid body modes of the overall gap covariance. This is useful when large "air gaps" are present in parts before production. The use of rigid body contributions is a way to account for rigid body closure of the parts prior to contact. These rigid body effects can be seen in Figure 3-12.

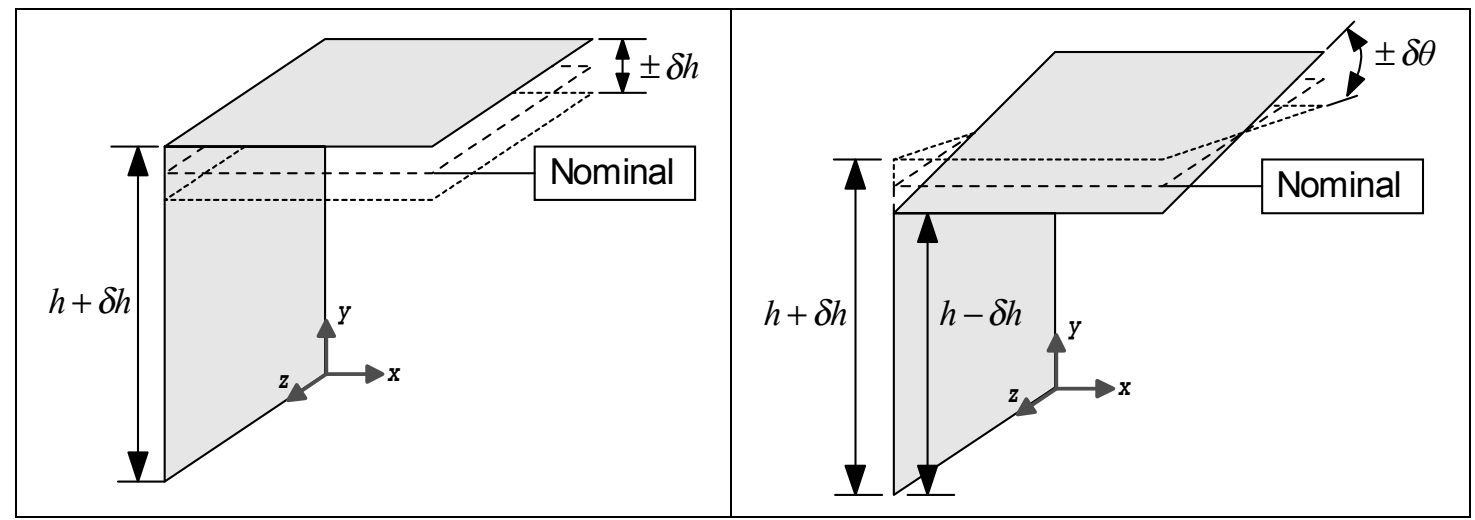

Figure 3-12: Rigid translational and rotational variations [Mortensen 2002]

Using the expected variations due to translation and rotation, $\left\{\sigma_{T}^{2}\right\}$ and $\left\{\sigma_{R}^{2}\right\}$, the covariances due to these effects can be calculated according to the equations:

$$
\begin{array}{lll}
\text { Translation: } & {\left[\Sigma_{\text {Trans }}\right]=\left[S(0)_{\mathrm{lg}}\right]\left[\Sigma_{T}\right]\left[S(0)_{\mathrm{lg}}\right]^{T}} & \text { Eq. 3-28 } \\
\text { Rotation: } & \left.\left[\Sigma_{\text {Rot }}\right]=\left[S(1)_{\mathrm{lg}}\right] \Sigma_{R}\right]\left[S(1)_{\mathrm{lg}}\right]^{T} & \text { Eq. 3-29 }
\end{array}
$$

Where $\left[S(0)_{\mathrm{lg}}\right]$ and $\left[S(1)_{\mathrm{lg}}\right]$ denote a zero-order and first-order Legendre transformation respectively. The matrices $\left[\Sigma_{T}\right]$ and $\left[\Sigma_{R}\right]$ are matrices created with the values of $\left\{\sigma_{T}^{2}\right\}$ and $\left\{\sigma_{R}^{2}\right\}$ populating the diagonals respectively. 
The whole process for estimating the gap covariance is given in Figure 3-13.

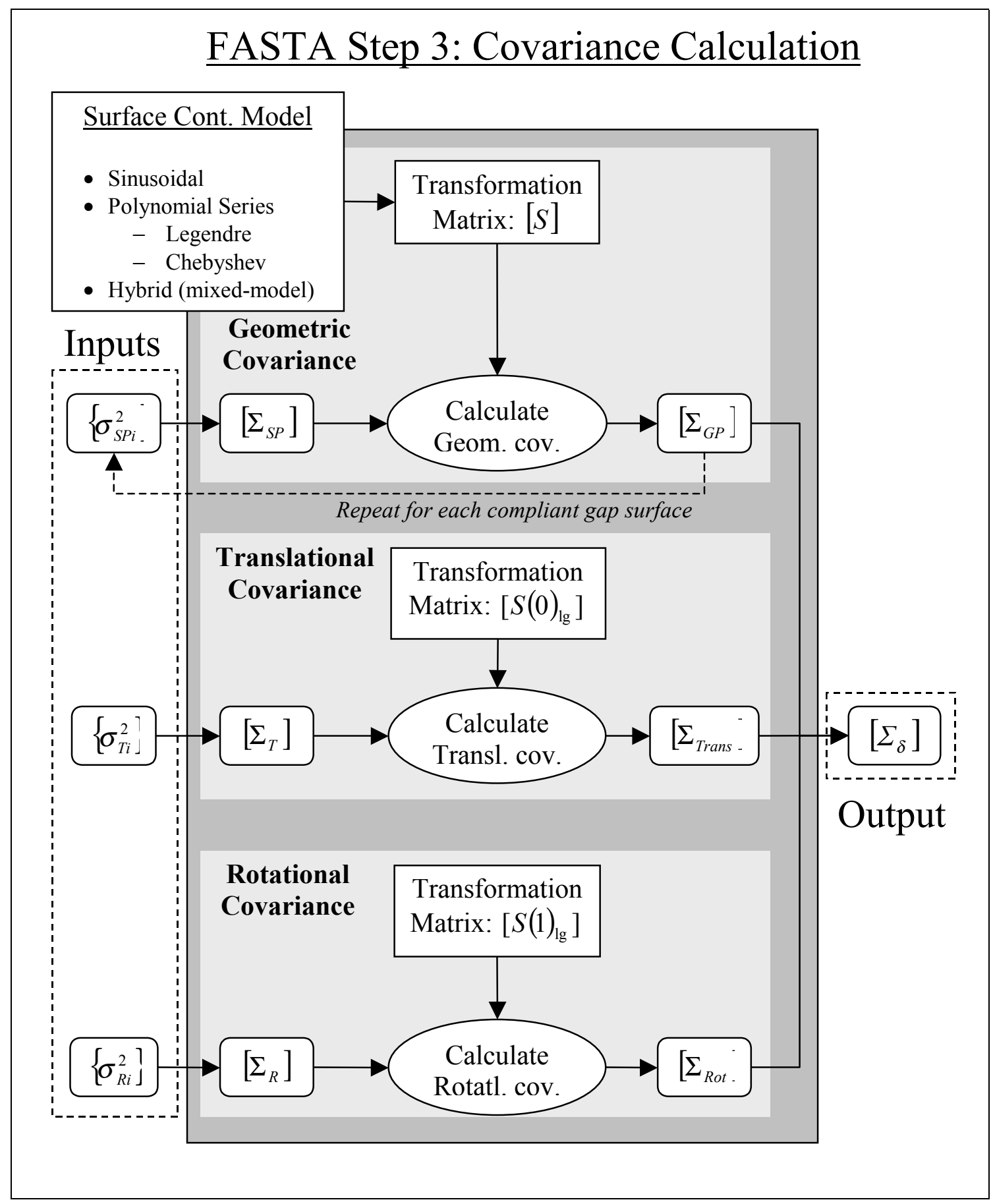

Figure 3-13: Process flow diagram for calculating gap covariance [Mortensen 2002] 


\subsection{Statistical FEA Solution}

With all the constitutive parts of both the mean and the covariant equation determined, a statistical solution may be calculated.

\subsubsection{Primary Solution}

The primary solution is finding the mean and variance of the assembly force. This is accomplished by solving Equations 3-30 and 3-31.

$$
\begin{array}{lll}
\text { Mean: } & \left\{\mu_{F}\right\}=\left\lfloor\mathbf{K}_{e q} \mid\left\{\mu_{\delta}\right\}\right. & \text { Eq. 3-30 } \\
\text { Covariance: } & \left.\left[\Sigma_{F}\right]=\left[\mathbf{K}_{e q}\right] \Sigma_{\delta}\right]\left[\mathbf{K}_{e q}\right]^{T} & \text { Eq. 3-31 } \\
\text { where: } & {\left[\Sigma_{\delta}\right]=\left[\Sigma_{G A}\right]+\left[\Sigma_{G B}\right]+\left[\Sigma_{T}\right]+\left[\Sigma_{R}\right]} & \\
& {\left[\Sigma_{G A}\right]=\left[S_{A}\right]\left[\Sigma_{A}\right]\left[S_{A}\right]^{T}} \\
& {\left[\Sigma_{G B}\right]=\left[S_{B}\right]\left[\Sigma_{B}\right]\left[S_{B}\right]^{T}}
\end{array}
$$

These equations can be solved directly, since the mean and the covariance of the gap are known. The mean and covariance solutions are also obtained for the displacements and forces at all mating surfaces throughout the assembly.

\subsubsection{Part Solutions and Post-Processing}

To obtain force and displacement predictions at interior nodes of the parts, the boundary displacements from the primary solution may be applied to the individual parts. Each part is analyzed independently. The purpose of this analysis is to determine $\left\{\mu_{\delta_{i}}\right\}$ and 
$\left\lfloor\Sigma_{\delta_{i}}\right\rfloor$, the mean and the covariance of the deflection of the interior nodes. To do this, $\left\{\mu_{\delta_{b}}\right\}$ and $\left[\Sigma_{\delta_{b}}\right\rfloor$, the deflection and covariance of the boundary nodes determined from the primary FASTA solution are applied to the original, unreduced stiffness matrix for each part and solved directly for the interior force and displacements.

A more difficult solution is to find the internal stress throughout the individual parts due to the assembly force. Internal stress solutions may be more important to a designer than deflection solutions, since high stresses can shorten part life by leading to unacceptable fatigue failure, plastic deformation or stress corrosion damage. Using the constitutive $[D]$ and kinematic $[B]$ matrices obtained from the FEA package, the individual part stresses may be found by applying Equations 3-32 and 3-33.

$$
\begin{array}{ll}
\text { Mean: } & \left\{\mu_{\sigma}\right\}=\left[D \rrbracket[B]\left\{\mu_{\delta}\right\}\right. \\
\text { Variance: } & {\left[\Sigma_{\sigma}\right]=[D][B]\left[\Sigma_{\delta} \llbracket[B]^{T}[D]^{T}\right.}
\end{array}
$$

Bihlmaier [1999] found that the mean stress solution may be determined element-byelement, as in classical FEA. However, because of covariance between nodes, the variance of the stress must be solved globally for each part, thus taking into account all interdependence. Current FEA programs do not have this capability, so such a solution must be set up and solved by an external process. The solution of equation 3-33, because of the nodal interdependence, will take greater calculation power. But, because only one part is analyzed at a time, with specified boundary conditions from the assembly solution, the problem size is held to a minimum. The difficult task is mapping $[D]$ and $[B]$ matrices for each element into the global $[D]$ and $[B]$ matrices for the part. 
The full diagram for this final step in the FASTA method is shown in Figure 3-14.

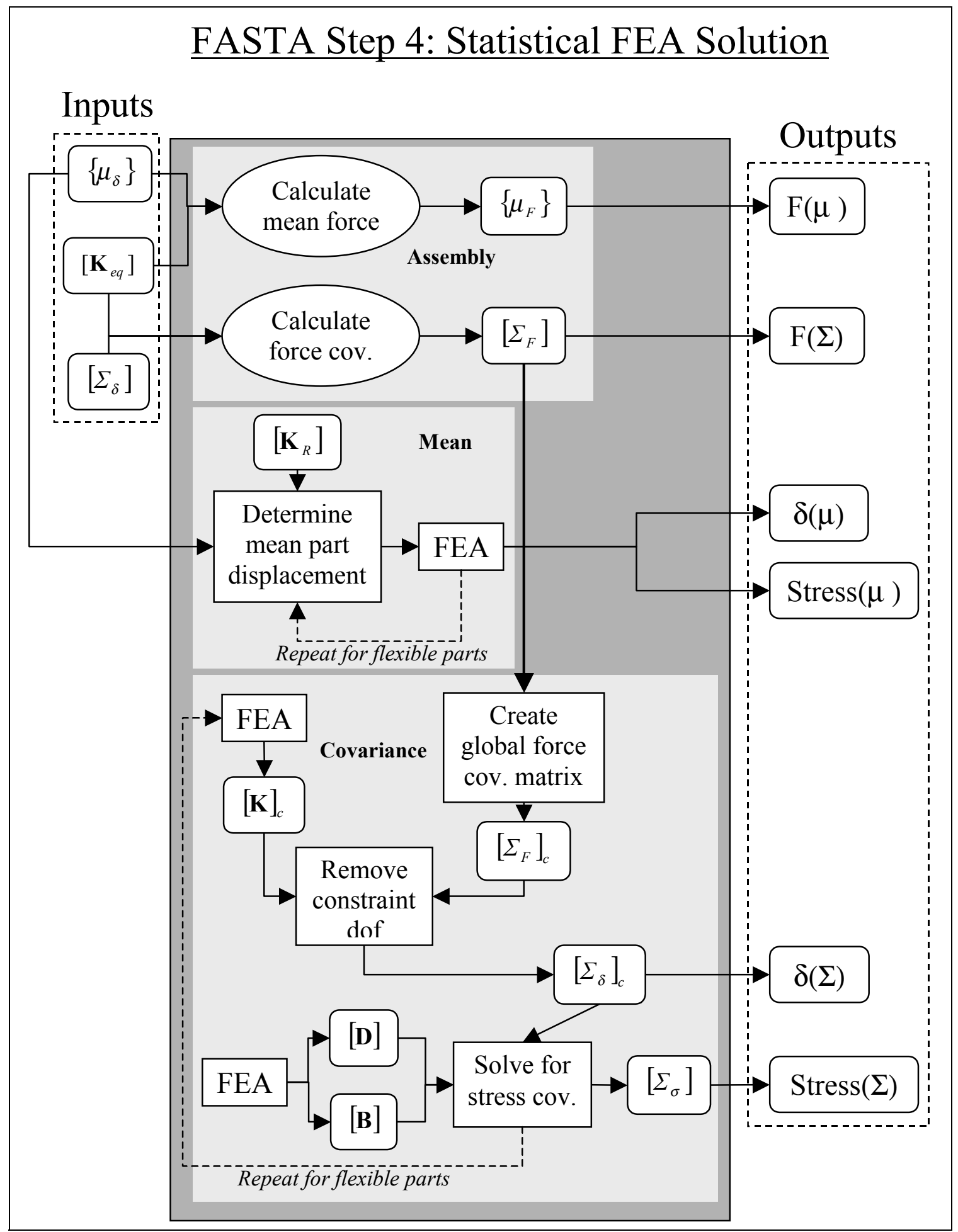

Figure 3-14: Process flow diagram for statistical FEA solution [Mortensen 2002] 


\section{Chapter 4: $\quad$ Fixtured Assembly Analysis}

This chapter develops the methods which may be used when analyzing flexible assemblies which incorporate a fixture. As stated previously, the current FASTA method cannot fully predict the PCFR cycle of a fixtured assembly process. This difference is again shown in Figure 4-1.

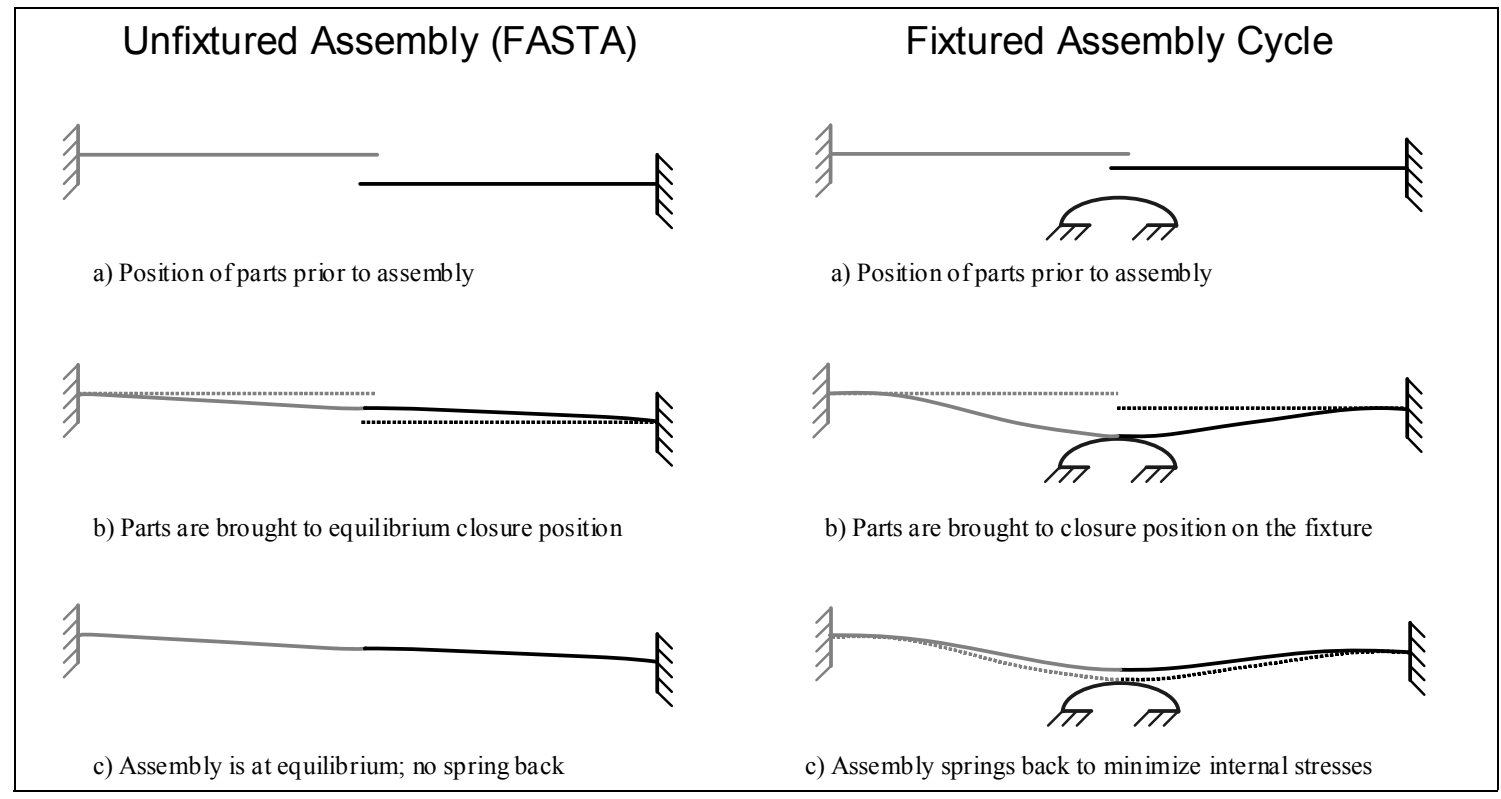

Figure 4-1: Flexible assembly deformations with and without the use of a fixture

If the parts and fixture were manufactured perfectly, each part would be flush against the fixture in the placement stage. However, the parts, the fixture, or all may have inherent misalignment, which would create a gap between the fixture and the part. From the above illustrations, the final shape of the assembly predicted by the FASTA method will 
differ from the final shape of an actual fixtured assembly. Even if the parts are brought to an arbitrary junction using the FASTA method, the assembly will spring back to the above shape. This is because FASTA analysis does not depend upon the initial position of the junction, but only on the final force-equilibrium position. It is apparent a solution for a fixtured analysis will require a multiple step solution which differs from the current FASTA method.

The limitations of the FASTA method include:

- Assumes the final state of deformation is independent of fixtures.

- Neglects changes in part length due to slip joints during clamping.

- Neglects membrane stresses, or "arch effects", due to large deformations.

- Neglects the effects of inelastic material behavior, which inhibits full springback.

- Assumes deformations are small, such that incremental updates of the stiffness matrix are not required.

- Neglects the effects of assembly contacts which might occur between specified closure points.

The analysis method is simplified greatly with the assumption that the stiffness matrix is constant over the range of deflection and that no mid-node contact occurs at any time. The small deformation assumption is reasonable for many assembly processes, where the members remain elastic throughout their ranges of deformation. Therefore, developing an analysis method to address the final three points is not within the scope of this thesis. 
The method developed in this thesis addresses the first three points only. The new fixtured analysis method must be equivalent to FASTA in the following ways:

- Linear analysis (constant stiffness throughout the range of deflection)

- Accurate for both force-closure and force-and-moment-closure assemblies

- Provides a stochastic solution

Two ways in which these components may be analyzed are by elastic superposition, introduced in Sec. 4.2 and by piecewise-linear elastic analysis, introduced in Sec. 4.3.

\subsection{Method Assumptions}

The new analysis method is solved stepwise, as a sum of linear deformations, simulating the steps in the PCFR process. Consider a simple example involving the fixtured welding of two sheet-metal components, as shown in Figures 4-2 to 4-5. Steps $1 \& 2$ of the new analysis method involve analyzing the placement and clamping of the components to the fixture. In Step 3, the two components are fastened together, creating an assembly. In Step 4, they are released from the fixture, allowing springback. Determining the final shape involves the combination of the results of steps 1-4 to obtain the overall solution for the assembly.

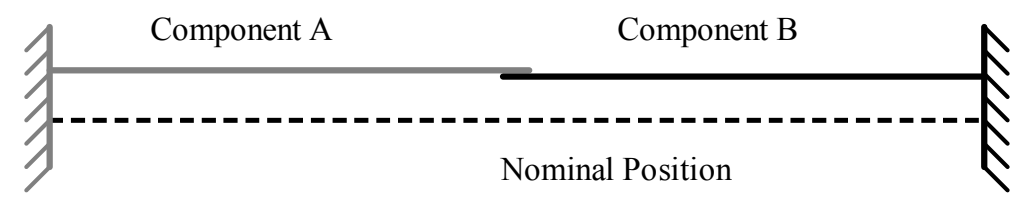

Figure 4-2: Two components after the placement stage of an assembly process 


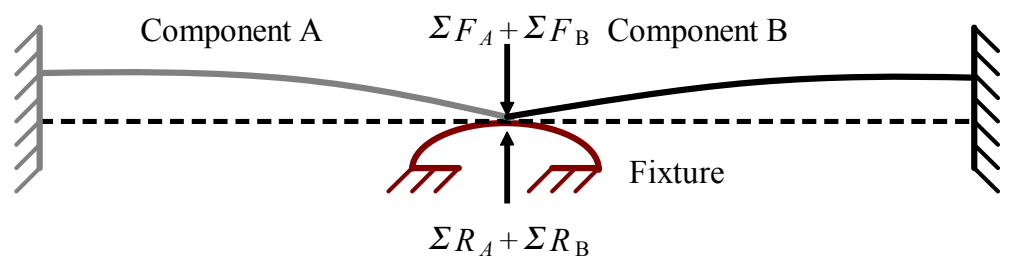

Figure 4-3: Components $A$ and $B$ are closed (clamped) against the fixture

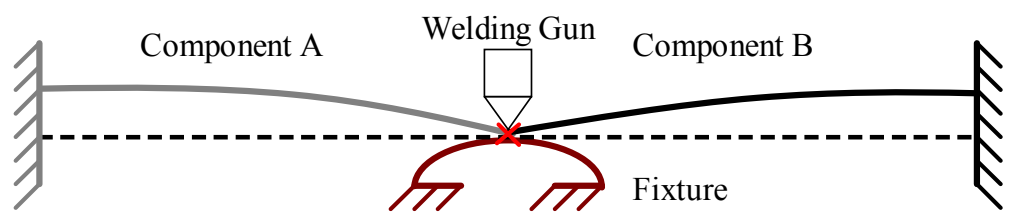

Figure 4-4: Components $A$ and $B$ are fastened together

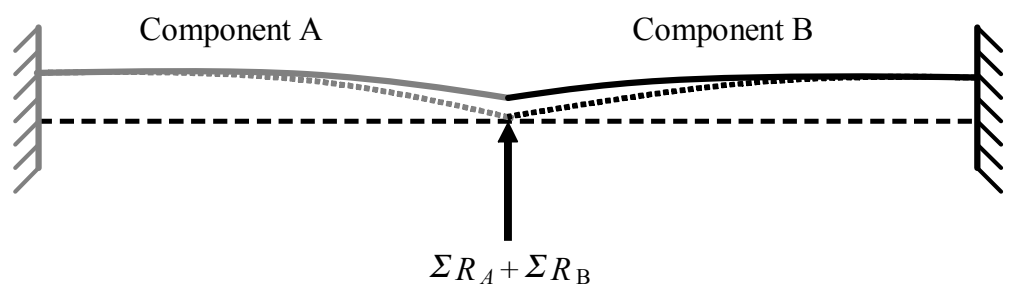

Figure 4-5: The assembly of $A$ and $B$ exhibits springback upon release from the fixture

Fundamental to the applications of these analysis methods is the assumption of elastic material behavior. This means the internal stresses due to the closure force can readily return each beam to its initial, unstressed position upon release. After assembly and release, however, the combined beams will seek a new equilibrium position. Additionally, if two or more independent forces or moments work on one body, and neither exceeds the elastic limit of the body, the forces and moments can be analyzed 
independent of each other and the resulting deflections and stresses added by superposition, as shown in Roark [1965]. This property becomes significant when analyzing the fixturing and assembly of two parts.

\subsection{Elastic Superposition}

The simplest method for modeling this multi-step operation involves linear elastic superposition. Elastic superposition involves superimposing the solutions for several loads cases, which simulate all assembly steps. One advantage to the elastic superposition method is the existence of statically determinate or closed-form solutions. An example using elastic superposition involving closed-form solutions follows.

\subsubsection{Elastic Superposition Theory}

Consider a cantilever beam which, due to misalignment, does not contact an assembly fixture. The part could be forced onto the fixture by application of a force $F$ to the free end, as seen in Figure 4-6.

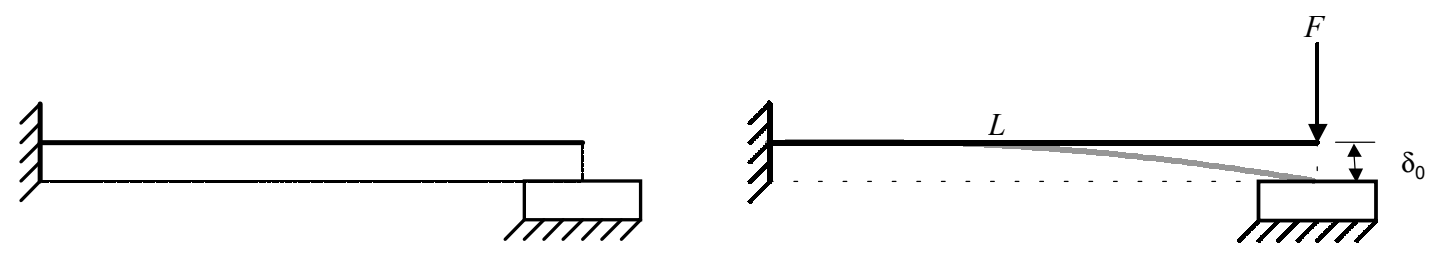

Figure 4-6: A misaligned cantilever beam and fixture

If the deflection $\delta_{0}$ is small compared to the length $L$, the closure force can be related to the deflection by Equation 4-1, derived from simple beam theory. 


$$
F=\frac{3 E I}{L^{3}} \delta_{0}
$$

Eq. 4-1

Where $\mathrm{E}$ is the Young's modulus and I is the area moment of inertia of the beam. The $y$ coordinates of the deformed shape may be obtained from Equation 4-2.

$$
y=\frac{-F x^{2}}{6 E I}(3 L-x)
$$

Where $x$ is the distance along the beam measured from the wall. The resulting shape of the beam will resemble Figure 4-7.

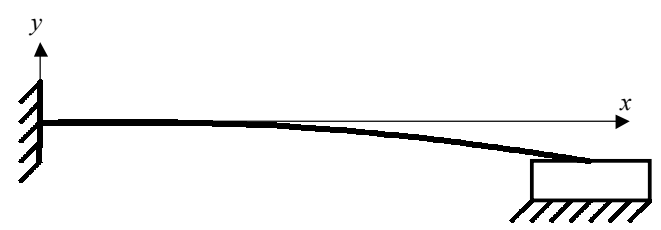

Figure 4-7: Resulting shape of the beam when force-closed

If the stiffness does not change throughout its deflection, a valid assumption based upon small tolerance-to-part length ratios, it is assumed the beam will return to its original shape upon release. It is further assumed that this springback can be modeled as a reaction, equal in magnitude and opposite in direction, applied at the same junction as the closure force.

Force-and-moment-closed assemblies can differ slightly from force-closed assemblies, due to the conflicting effects of forces and moments. An example of this phenomenon is shown in Figure 4-8. 


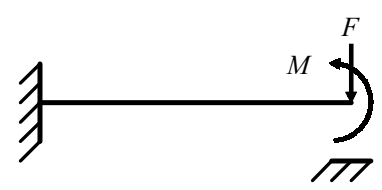

Figure 4-8: Force and moment acting on end of a beam

Consider the force and moment acting on the cantilever independently of each other. The closure force reduces the gap between the beam and the fixture. As a result, the end of the beam rotates clockwise. The closure moment rotates the end of the beam counterclockwise, but increases the gap between the beam and the fixture. This effect can be modeled using simple beam theory as well. When a force and moment are used to close the same gap $\delta_{0}$, and bring the end of the beam to angle $\alpha$, the force $F$ and moment $M$ are found according to Equations 4-3 and 4-4.

$$
\begin{gathered}
F=\frac{-6 E I}{L^{2}} \cdot\left(\frac{2 \delta_{0}}{L}+\alpha\right) \\
M=\frac{-2 E I}{L} \cdot\left(\frac{3 \delta_{0}}{L}+2 \alpha\right)
\end{gathered}
$$

If the angle $\alpha$ is zero radians, meaning the final angle of the end of the beam is the same as the initial angle, the force-and-moment closure force is equal to four times the forceonly closure force.

\subsubsection{Elastic Superposition Assembly Processes}

Elastic superposition can be applied to an assembly of parts to simulate the assembly process. Consider two misaligned beams, brought together onto a fixture and then welded together. In the placement stage, each component is constrained from motion by 
boundary conditions, simulating the component in its placed position. During the clamping stage, each component is closed in turn against the fixture by assembly forces and assembly moments. Chang [1996] defines a welding gun as an example of a machine which can force-close misaligned parts. In this step, the distance between the fixture and the point of closure is found and, using a force-displacement relationship, the required closure force is determined. After the clamping stage, the components are fastened together at their deformed positions. The weld nugget, rivet, adhesive bond, or threaded fastener transmits both transverse and shear loads between the components, as shown in Liu [1997]. At this stage, the assembled components exhibit a new stiffness relationship, represented in elastic superposition as a single, continuous beam, supported by fixed ends.

To apply elastic superposition to the assembly, two modeling assumptions must be made to analyze the springback step. The first assumption is that we can add reaction forces linearly at the junction of the two components. If this assumption is made, the equivalent force inducing the springback can easily be determined from the assembly forces. The second assumption is that this springback force can be applied to the combined, undeformed components. The resulting deflection is then added to the clamped deflection to simulate springback. It is assumed that the stiffness of the assembly will be much stiffer than that of the individual components. If this assumption is made, the final position will be easier to calculate, since it relies upon the initial stiffness calculation of each component. 


\subsubsection{Closed-form Elastic Beam Equations}

To verify elastic superposition, simplified beam theory will be used since it yields statically-determinate solutions for simple load and geometry configurations. While most designs are not so simple, closed-form solutions can be used to verify our theory without having to resort to FEA analysis. Examples of closed-form equations from elemental beam theory which concern our simple assembly are listed in Table 4.1. 
Table 4.1: Closed-form beam equations [Roark 1965]

\begin{tabular}{|c|c|c|}
\hline Description & Illustration & Position as a function of $x$ \\
\hline $\begin{array}{l}\text { Left end } \\
\text { cantilever } \\
\text { with end } \\
\text { load }\end{array}$ & 才 & $y=\frac{-F x^{2}}{6 E I}(3 L-x)$ \\
\hline $\begin{array}{l}\text { Right end } \\
\text { cantilever } \\
\text { with end } \\
\text { load }\end{array}$ & $y+$ & $y=\frac{-F}{6 E I}\left(x^{3}-3 L^{2} x+2 L^{3}\right)$ \\
\hline $\begin{array}{l}\text { Left end } \\
\text { cantilever } \\
\text { with end } \\
\text { couple }\end{array}$ & 素 & $y=\frac{-M x^{2}}{2 E I}$ \\
\hline $\begin{array}{l}\text { Right end } \\
\text { cantilever } \\
\text { with end } \\
\text { couple }\end{array}$ & $\overbrace{\stackrel{M}{\longrightarrow}}^{M}$ & $y=\frac{M}{2 E I}\left(L^{2}-2 L x+x^{2}\right)$ \\
\hline $\begin{array}{l}\text { Both ends } \\
\text { fixed, } \\
\text { intermediate } \\
\text { load }\end{array}$ & $y_{\mathrm{A}}^{\mathfrak{X}_{\mathrm{B}}^{y}}$ & $\begin{array}{c}y_{A \rightarrow B}=\frac{F b^{2} x^{2}}{6 E I L^{3}}(3 a x+b x-3 a L) \\
y_{B \rightarrow C}=\frac{F a^{2}(L-x)^{2}}{6 E I L^{3}}[(3 b+a)(L-x)-3 b L]\end{array}$ \\
\hline $\begin{array}{l}\text { Both ends } \\
\text { fixed, } \\
\text { intermediate } \\
\text { couple }\end{array}$ & 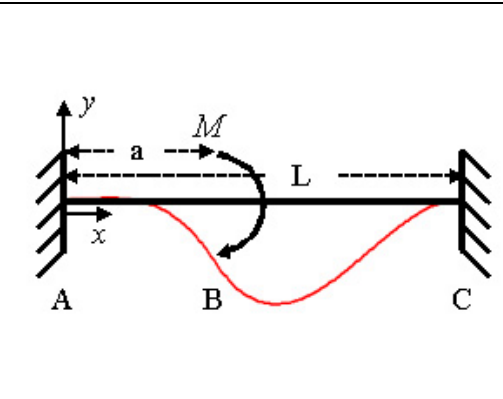 & $\begin{array}{c}R_{1}=\frac{-6 M}{L^{3}}\left(L a-a^{2}\right) \\
M_{1}=\frac{-M}{L^{2}}\left(4 L a-3 a^{2}-L^{2}\right) \\
y_{A \rightarrow B}=\frac{-1}{6 E I}\left(3 M_{1} x^{2}-R_{1} x^{3}\right) \\
y_{B \rightarrow C}=\frac{1}{6 E I}\left[\left(M-M_{1}\right)\left(3 x^{2}-6 L x+3 L^{2}\right)-R_{1}\left(3 L^{2} x-x^{3}-2 L^{3}\right)\right]\end{array}$ \\
\hline
\end{tabular}




\subsubsection{Elastic Superposition Verification}

Two assembly cases will be analyzed by using elastic superposition, force-closure and force-and-moment-closure. The verification of this analysis was completed on Mathcad Professional.

\subsubsection{Force-Closed Assemblies}

Force-closed assemblies are the simplest assemblies to analyze, because it is assumed moments in the clamping and release stages will be zero. This reduces the number of independent solutions to three; one solution for the initial deflection of each beam and one solution for the force-only springback. For verification, the force-closure assembly beam properties will be as follows:

Table 4.2: Beam properties for unequal length, equal offset analysis, force-closure

\begin{tabular}{|l|l|l|l|l|}
\hline & E & I & Beam Length & Offset $(\delta)$ \\
\hline Beam 1 & $2.9 \times 10^{7} \mathrm{psi}$ & $4.167 \times 10^{-5} \mathrm{in}^{4}$ & 4 in & 0.25 in \\
\hline Beam 2 & $2.9 \times 10^{7} \mathrm{psi}$ & $4.167 \times 10^{-5} \mathrm{in}^{4}$ & 8 in & 0.25 in \\
\hline
\end{tabular}

Step 1 is to force the beams in contact with the fixture. This is done with downward forces of $14.16 \mathrm{lb}_{\mathrm{f}}$ on the left beam and $1.77 \mathrm{lb}_{\mathrm{f}}$ on the right beam. The resulting beams are bent down as shown exaggerated in Figure 4-9. 


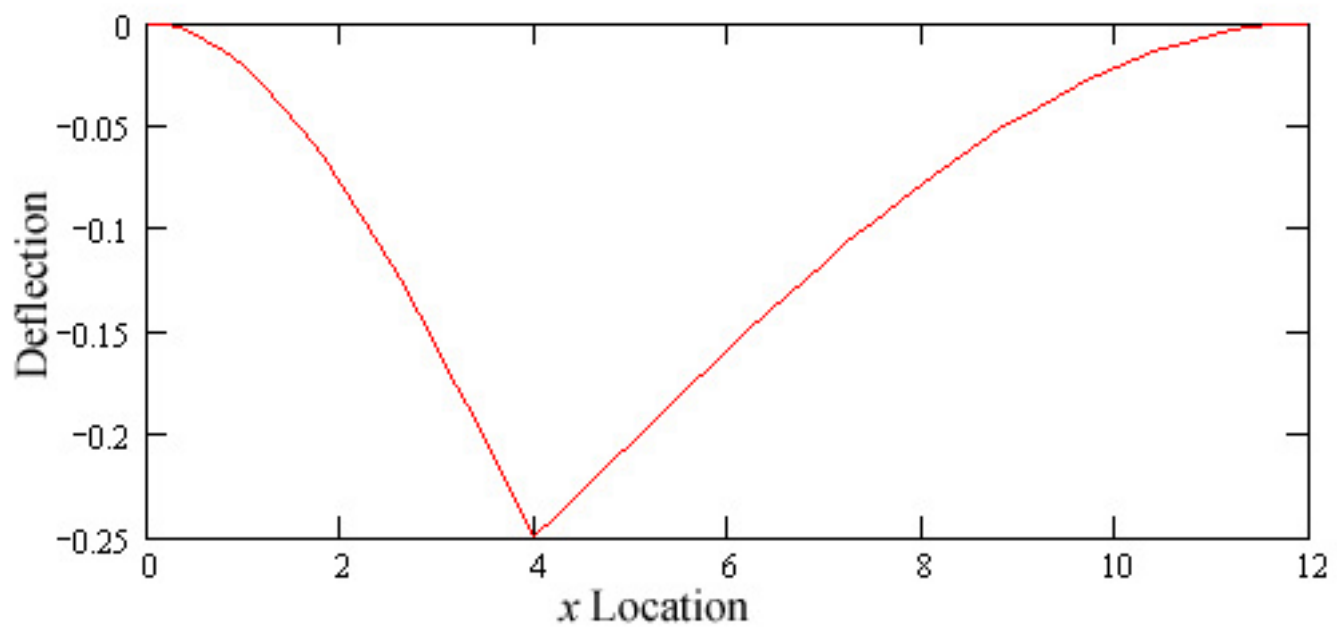

Figure 4-9: Beams in clamped position

Because we assumed both beams had identical moments of inertia, we are able to use the equation for an intermediate load applied to two cantilevers. The load is the positiveacting sum of the closure forces from the first step, or $15.93 \mathrm{lb}_{\mathrm{f}}$. The resulting springback deflection is shown in exaggerated form in Figure 4-10.

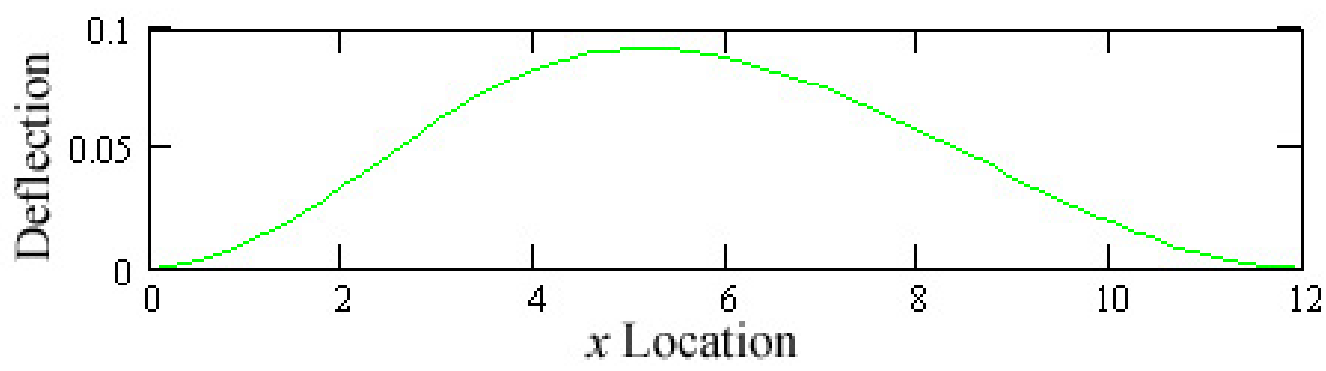

Figure 4-10: Springback from nominal position

The superposition of these two solutions produces the final position after springback. In Figure 4-11, each step is shown along with superposition. Using the equations given in table 4.1 for this case, we find that the final position for the junction of the beams is 0.167 inches downward, or ${ }^{2} / 3$ the original gap. 


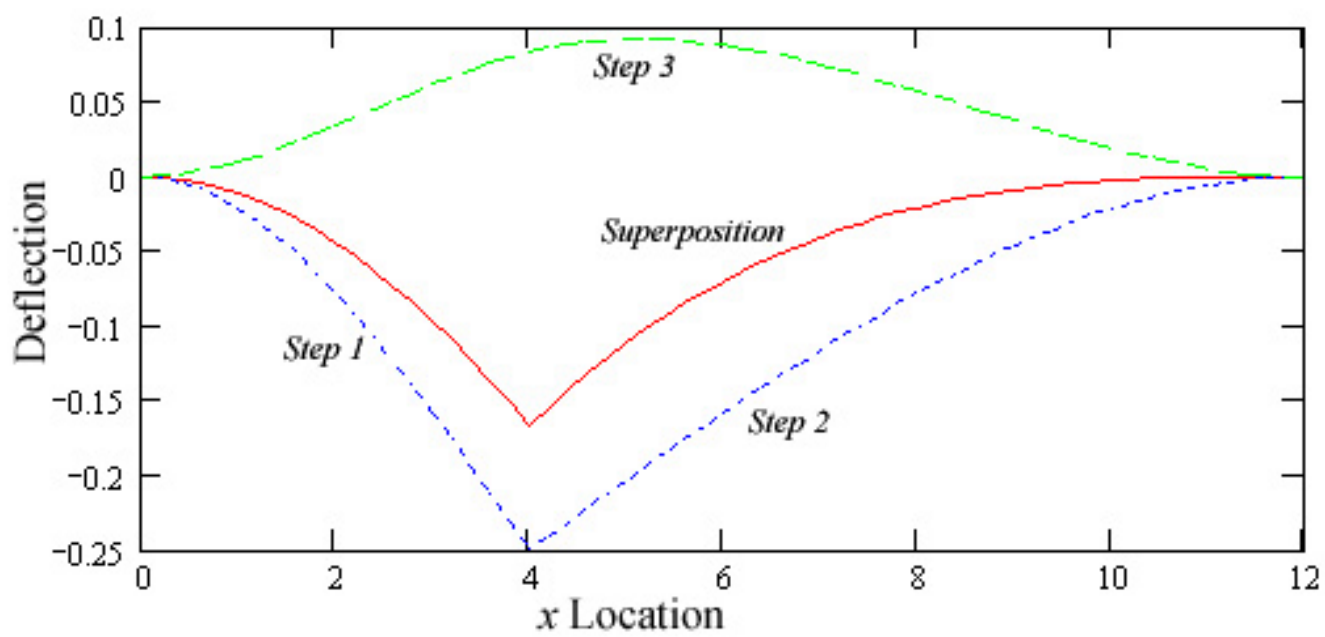

Figure 4-11: Final positions of assembly

\subsubsection{Force- and Moment-Closed Assemblies}

Most production joining operations rely upon force and moment closure. Often, it is not enough simply to bring the components into contact with each other; they must be in contact and exhibit tangency. In riveting operations, each mating surface has an overlapping flange which forces tangency to the mating part. The wide flange is necessary to confine the rivet to its hole. In welding operations, the weld nugget forms at the junction of the two parts, and the greater the tangency, the larger the junction and stronger the weld. The analysis method must also be verified on force-and-moment closures as well.

This large closure force adversely affects the solutions. Because elastic superposition assumes the stiffness is equivalent, we can expect to have a greater springback in forceand moment-closure for the same gap, as compared to force-closure. To demonstrate this 
effect, the force- and moment-closure assembly beam properties will be identical to the force-closure assembly beam properties:

Table 4.3: Beam properties for unequal length, equal offset analysis, force- and moment-closure

\begin{tabular}{|l|l|l|l|l|}
\hline & E & I & Beam Length & Offset $(\delta)$ \\
\hline Beam 1 & $2.9 \times 10^{7} \mathrm{psi}$ & $4.167 \times 10^{-5} \mathrm{in}^{4}$ & 4 in & 0.25 in \\
\hline Beam 2 & $2.9 \times 10^{7} \mathrm{psi}$ & $4.167 \times 10^{-5} \mathrm{in}^{4}$ & 8 in & 0.25 in \\
\hline
\end{tabular}

To bring the left beam to closure with the fixture, a downward force of $56.64 \mathrm{lb}_{\mathrm{f}}$ and counterclockwise moment of 113.28 in $\mathrm{lb}_{\mathrm{f}}$ is applied to the end. The right beam requires a downward force of $7.08 \mathrm{lb}_{\mathrm{f}}$ and clockwise moment of $28.32 \mathrm{in} \mathrm{lb}_{\mathrm{f}}$. Each closure force is exactly four times the force required in force-only closure, as predicted by the equations. When the beams are brought in contact and tangent to the fixture, they appear as in Figure 4-12.

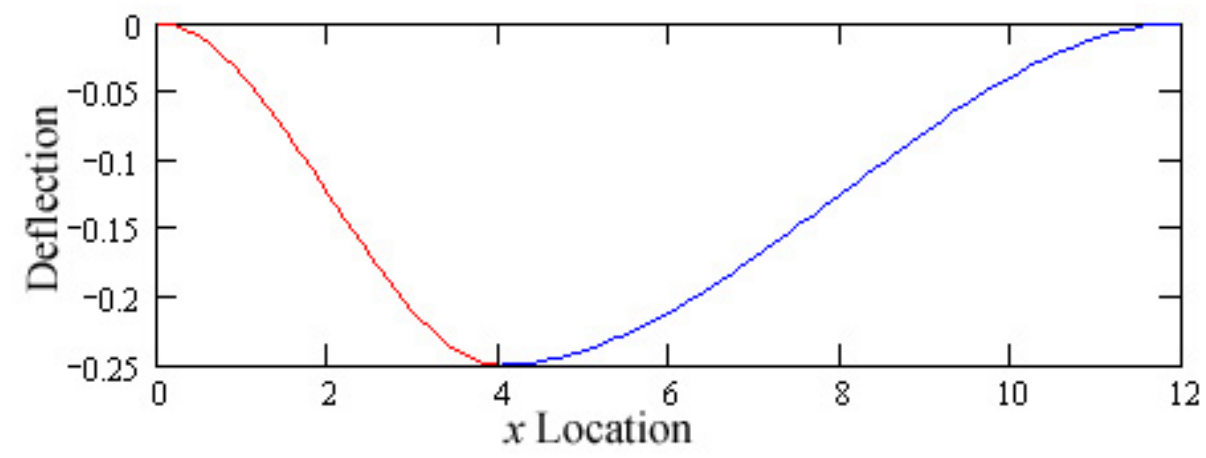

Figure 4-12: Force- and moment-closure of two beams

Two independent springback solutions are obtained, one for the combined forces and one for the combined moments. The force springback appears as in Figure 4-13. Notice the springback is larger than the clamping deflection of 0.25 in, due to the higher forces. 


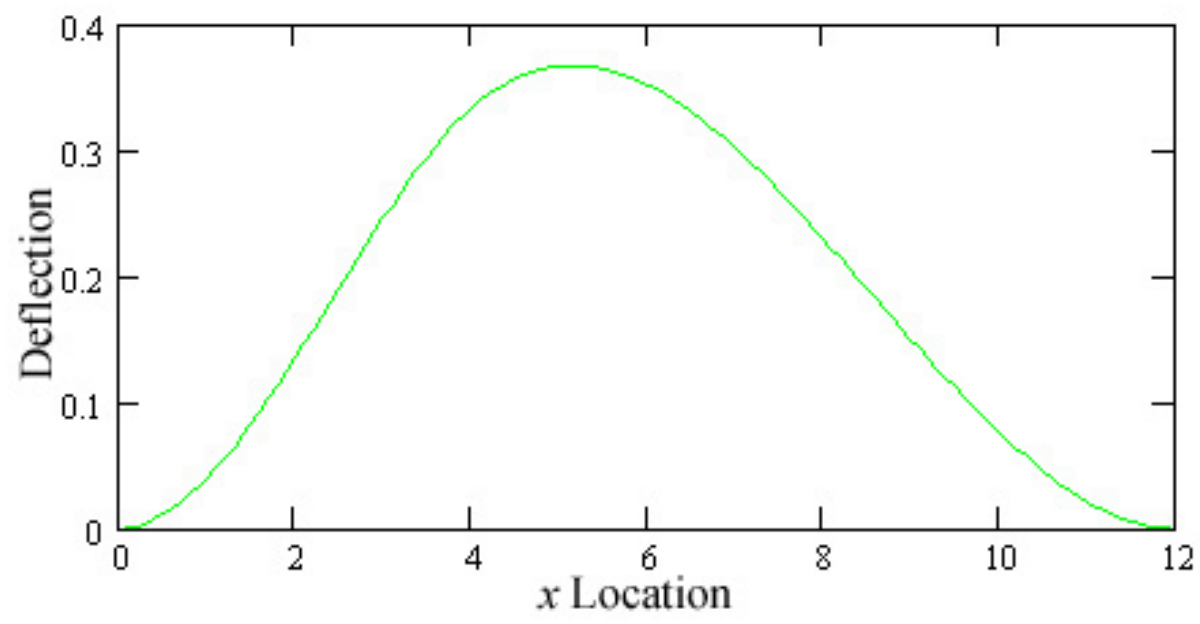

Figure 4-13: Force springback

When two beams have exactly the same length, moment of inertia, and modulus, the moment springback will be zero, since both moments will counteract each other. When the beams have different lengths, moduli of elasticity, or moments of inertia, springback due to reactive moments is expected. In the right beam, a moment was applied of 113.28 in $l b_{f}$ (counterclockwise), and in the left beam a moment was applied of -28.32 in $1 b_{f}$ (clockwise). The resulting reacting moment will be -84.96 in $\mathrm{lb}_{\mathrm{f}}$ (clockwise). The deflection resulting from the reactive moment springback is shown in Figure 4-14.

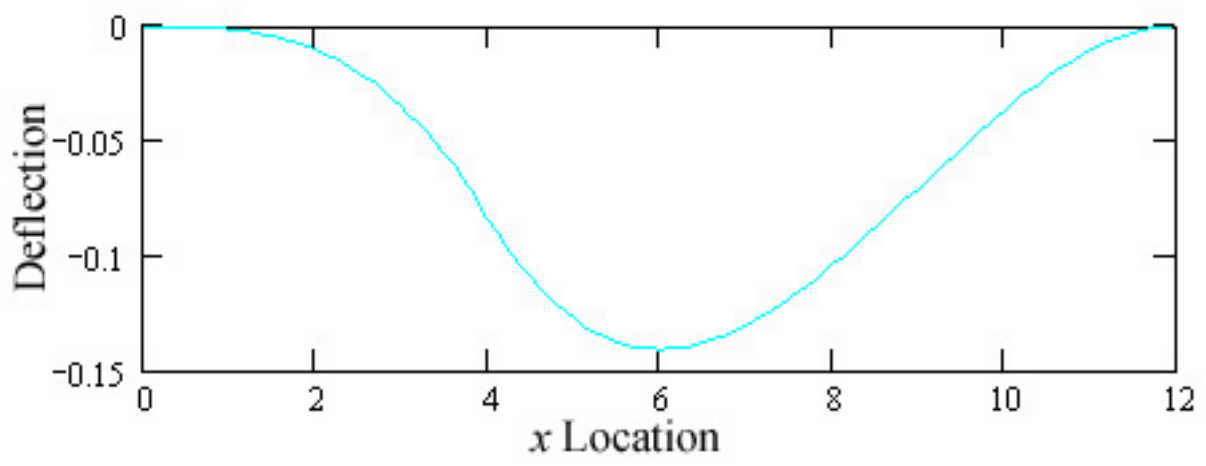

Figure 4-14: Moment springback 
Superimposing these six solutions (force and moment for each beam and force and moment springback) yields surprising results, as shown in Figure 4-15. The superposition of the solutions yields the final position of the assembly, which turns out to be the original position of the two beams, so there is no residual deformation due to misalignment during assembly. Furthermore, varying the fixture position does not affect the final position of the assembly as long as the beams remain elastic. This is due to simple beams not accounting for any axial loads.

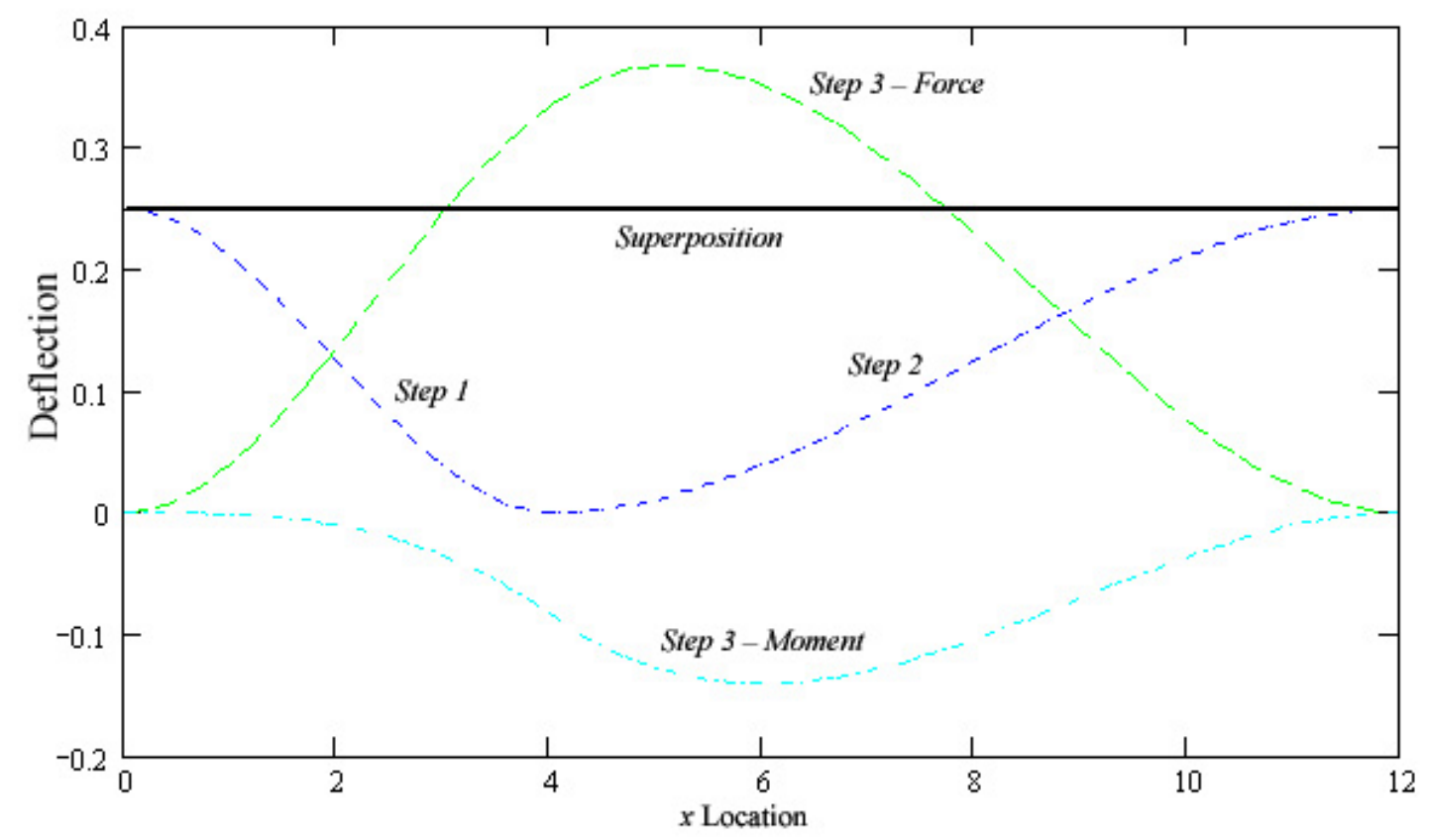

Figure 4-15: Force and moment superposition

If the two beams exhibited different offsets from the fixture, the final deformed assembly will always be between the two beams, at a ratio corresponding to their stiffnesses. In our case, when both beams exhibit the same offset from the fixture, the assembly returns to the original position of the beams, regardless of fixture position or difference in beam lengths, cross sections or materials. 


\subsubsection{Weaknesses of Elastic Superposition}

One of the weaknesses evident in elastic superposition is that the method is unable to account for the elongation of the neutral axis, which produces axial forces in the beam. This affects all results. The final position of the junction in force closure analysis will be some fraction of the distance between the beams and the fixture. The final position of the junction in force and moment closure analysis will always occur between the initial positions of the beams. The solutions given through elastic superposition will only be accurate for limited deflection. A more robust analysis method is needed, which is accurate for greater deflection ranges and includes elongation effects of the components. Piecewise-linear elastic analysis, introduced in the next section, can address these needs.

\subsection{Piecewise-Linear Elastic Analysis}

One of the fundamental assumptions of our analysis method is that in the ranges of the dimensional tolerances that produce alignment gaps, there exists a linear relationship between load and deflection. This means incrementing the load and recalculating the corresponding stiffness matrix at each step will not yield more accurate results.

However, to account for the axial loads, the new method presented here will start the springback analysis from the stiffness recalculated for the deformed geometry. This modified stiffness will still exhibit a linear force-to-deflection relationship. The springback solution can still be added by superposition to the clamping loads, since all the process steps are linear. Because this analysis is completed in three steps, each 
involving linear elastic relationships between force and deflection, this new technique will be called piecewise-linear elastic analysis, or PLEA for short.

The primary effect of the PCFR cycle is the elongation due to the slip joint created between overlapping surfaces. This change in length does not generate axial loads in the clamping step, because it is assumed the edges are unconstrained axially. However, in the springback step, both the length and the stiffness have changed. The part cannot return to its original position because the fasteners constrain it in its elongated position. Springback forces transferred between parts act in the axial direction, producing axial forces. Additionally, there is increased resistance to deformation. So, the stiffness has increased due to the deformed geometry and the occurrence of axial forces.

\subsubsection{Piecewise-Linear Elastic Analysis Theory}

PLEA accounts for the change in stiffness necessary to predict springback. The springback estimation equation which provides a basis for this method is mentioned in Camelio [2002], but is not demonstrated. PLEA is like elastic superposition analysis in all respects but two. It is anticipated these two differences will make PLEA a more realistic and robust analysis method than elastic superposition.

The first difference between PLEA and elastic superposition is that PLEA accounts for in-plane stiffness in all steps, due to elongation of the neutral plane. In beams, this produces axial stress; in shells or plates, it produces "membrane stress". This means that the part may have some in-plane stresses due to assembly to combine with bending stress. 
This also necessarily means that the analysis of any of the PCFR steps must be done with a commercial FEA program. While it is possible to compute the deformed shape of simple, planar elements after the clamping stage, using the deformed shape to calculate a new stiffness cannot be done in closed-form. Because our compliant assembly analysis will be implemented in a FEA package, this limitation is acceptable, since we are able use the outputs from the clamping stage as inputs for modeling the fastening stage. Since most parts have no closed-form solutions, the method cannot be checked using simple theory as we could with elastic superposition. The piecewise-linear elastic method must be verified on an assembly of real parts. This verification and the results are discussed in subsequent chapters.

The second difference between PLEA and elastic superposition is that, after the components are clamped to the fixture and their deformation is known, PLEA creates a new assembly stiffness matrix for the release step based upon the deformed position of the components. This difference will yield more realistic results than using the undeformed stiffnesses.

The deformed stiffness matrix assumption is possible due to the small deformations we are assuming. It is assumed in the clamping stage, each component deforms linearly with force. When the parts are fastened together and released from their deformed positions, the nonlinearity associated with the springback process is estimated by computing a new assembly stiffness in the deformed position. Once the new stiffness is determined, it will be assumed that the springback will occur linearly, just as the initial deflection did. This 
inherently nonlinear problem is approximated by solving and superimposing two piecewise-linear solutions.

When discussing springback, assembly toggle must be addressed. Toggle is cause by the existence of multiple states of stable equilibrium. A toggle action occurs when a force (in our case, the reaction forces) acts upon a structure, causing it to move through a position of instability to a new position of equilibrium. In sheet metal assembly, this is known as recoil or pop-out. PLEA cannot account for this nonlinear phenomenon. However, because PLEA does account for axial forces, some of the reaction force will be distributed axially, making the assembly stiffer in the transverse direction. This force distribution can be seen in Figure 4-16. This means the assembly will not return to its previous, undeflected state on its own. Because it cannot return to this state, it cannot toggle to another position of equilibrium.

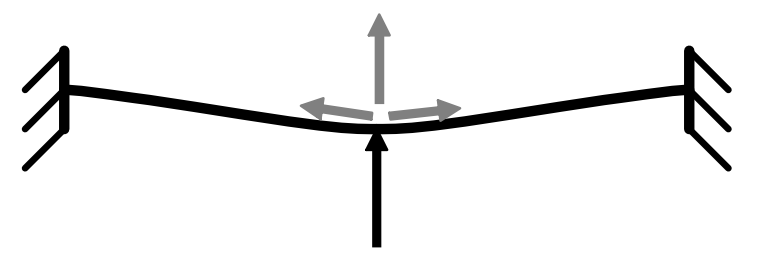

Figure 4-16: Axial and transverse springback components

\subsubsection{Piecewise-Linear Elastic Analysis Assembly Processes}

The PLEA method must also be implemented in the four steps illustrated above. In the first and second analysis steps, the components are displacement-closed to the fixture. The clamped shape and stress of the two components are determined by solving the fundamental FASTA equations: 
Mean Component Force: $\quad\left\{\mu_{F i}\right\}=\left[\mathbf{K}_{i}\right]\left\{\mu_{\delta i}\right\} \quad$ Eq. 4-5

Covariance of the Force: $\quad\left[\Sigma_{F i}\right]=\left[\mathbf{K}_{i}\right]\left[\Sigma_{\delta i}\right]\left[\mathbf{K}_{i}\right]^{T} \quad$ Eq. 4-6

Mean Component Stress: $\quad\left\{\mu_{\sigma i}\right\}=\left[D_{i}\right]\left[B_{i}\right]\left\{\mu_{\delta i}\right\} \quad$ Eq. 4-7

Covariance of the Stress: $\quad\left[\Sigma_{\sigma i}\right]=\left[D_{i} \mathbb{\|}\left[B_{i} \rrbracket\left[\Sigma_{\delta i} \mathbb{\|}\left[B_{i}\right]^{T}\left[D_{i}\right]^{T} \quad\right.\right.\right.$ Eq. 4-8

Where each component $i$ has its own initial gap $\left\{\delta_{i}\right\}$ from the fixture, dimensional and surface variations. The resulting forces and stresses are then computed from the stiffness $[\mathbf{K}]$, constitutive $[B]$, and kinematic $[D]$ relationships. We then use the results from the first two steps in the next analysis step.

Because we are assuming reaction forces are sums of the initial assembly forces, we can add the complements of the mean assembly forces for each node and model this as the springback analysis input, as shown in Equation 4-9.

$$
\left\{\mu_{F A}\right\}=\left\{-\mu_{F 1}\right\}+\left\{-\mu_{F 2}\right\}
$$

Eq. 4-9

Modeling the variation of the springback due to the variation in the component assembly force is done much the same way. Variances are additive and always greater than zero. The covariance of the springback force is given in Equation 4-10.

$$
\begin{aligned}
{\left[\Sigma_{F A}\right] } & =\left[\Sigma_{F 1}\right]+\left[\Sigma_{F 2}\right] \\
& =\left[\mathbf{K}_{1}\right]\left[\Sigma_{\delta 1}\right]\left[\mathbf{K}_{1}\right]^{T}+\left[\mathbf{K}_{2}\right]\left[\Sigma_{\delta 2}\right]\left[\mathbf{K}_{2}\right]^{T}
\end{aligned}
$$

Eq. 4-10

The assembly of the two parts in their deformed positions is then modeled in the FEA program to obtain its combined stiffness, kinematic and constitutive matrices. Using the relations given in Equations 4-9 and 4-10, the fundamental equations for the assembly springback can be written: 
Mean Assembly Deflection: $\quad\left\{\mu_{\delta A}\right\}=\left[\mathbf{K}_{A}\right]^{-1}\left\{\mu_{F A}\right\} \quad$ Eq. 4-11

Covariance of the Deflection: $\quad\left[\Sigma_{\delta A}\right]=\left[\mathbf{K}_{A}\right]^{-1}\left[\Sigma_{F A}\right]\left[\mathbf{K}_{A}\right]^{T^{-1}} \quad$ Eq. 4-12

Mean Assembly Stress: $\quad\left\{\mu_{\sigma A}\right\}=\left[D_{A}\right]\left[B_{A}\right]\left\{\mu_{\delta A}\right\} \quad$ Eq. 4-13

Covariance of the Stress: $\quad\left[\Sigma_{\sigma A}\right]=\left[D_{A}\right]\left[B_{A}\right]\left[\Sigma_{\delta A}\right]\left[B_{A}\right]^{T}\left[D_{A}\right]^{T} \quad$ Eq. 4-14

The final shape of the assembly is determined after the mean and covariance of the springback deflection are calculated. The final shape is the sum of the clamping and release deformations. The final stresses of the assembly are determined by summing the stresses of each individual solution node-by-node. These analysis steps to solve for the mean solution are demonstrated in the following section.

\subsubsection{PLEA Processes Verification}

The PLEA method was verified using the assembly of two beams. These analyses are similar to the force-and-moment closure performed previously, but unlike elastic superposition analysis, the PLEA method uses the deformed stiffness in the springback analysis, and in-plane stiffness is assumed. Because of this, different and more realistic results were expected.

In the first analysis step, one component of the assembly, Beam 1, is modeled in ANSYS $^{\circledR}$ and linear and angular displacement loads are applied to it. It is modeled with 16 three-node beam elements, corresponding to one three-node beam element every $1 / 4$ inch. The loads are applied to the free end to bring that end in contact with and tangent to 
the fixture. The nodal deflections are obtained directly from ANSYS ${ }^{\circledR}$ as tables of nodal solutions. The stresses due to assembly at each node can also be obtained, if desired.

The second step involves applying displacement and angular displacement loads to the end of the second component, Beam 2, to bring it into contact with and tangent to the fixture, as in step 1. The nodal deflections are again obtained from ANSYS ${ }^{\circledR}$ in tabular form. The nodal stresses due to assembly can also be obtained, if desired.

For the third and final step of the analysis, the deflected nodal positions from steps 1 and 2 are entered directly into ANSYS ${ }^{\circledR}$ and the whole assembly is meshed. The assembly forces and moments are added and applied to the junction node as reactions and the deflection equations are solved. Once the solution is obtained, the nodal deflections are obtained from ANSYS ${ }^{\circledR}$ in tabular form. The theoretical final shape of the assembly is the final shape obtained after this last step, and it is plotted in $\operatorname{Excel}^{\circledR}$ and compared to solutions obtained from elastic superposition. If the assembly stresses are desired, the nodal stresses obtained from this step are added node-by-node to the results obtained from analysis steps 1 and 2. Because stresses can not be verified in these examples, they will not be obtained from ANSYS ${ }^{\circledR}$.

\subsubsection{Two Beam Assembly, Equal Length, Equal Offset}

One of the simplest assemblies to verify is one in which the beams have the same properties and lengths, and the fixture exhibits position error. Symmetry cancels out the 
influence of the moment reaction, yielding force-only reaction. The analysis was setup as listed on Table 4.4.

Table 4.4: Beam properties for equal length, equal offset analysis

\begin{tabular}{|l|l|l|l|l|}
\hline & E & I & Beam Length & Offset $(\delta)$ \\
\hline Beam 1 & $2.9 \times 10^{7} \mathrm{psi}$ & $4.167 \times 10^{-5} \mathrm{in}^{4}$ & 6 in & 0.25 in \\
\hline Beam 2 & $2.9 \times 10^{7} \mathrm{psi}$ & $4.167 \times 10^{-5} \mathrm{in}^{4}$ & 6 in & $0.25 \mathrm{in}$ \\
\hline
\end{tabular}

The graph below shows the positions of both beams in the clamped (before springback) stage in blue, and the results after springback of both PLEA (green) and elastic superposition (brown).

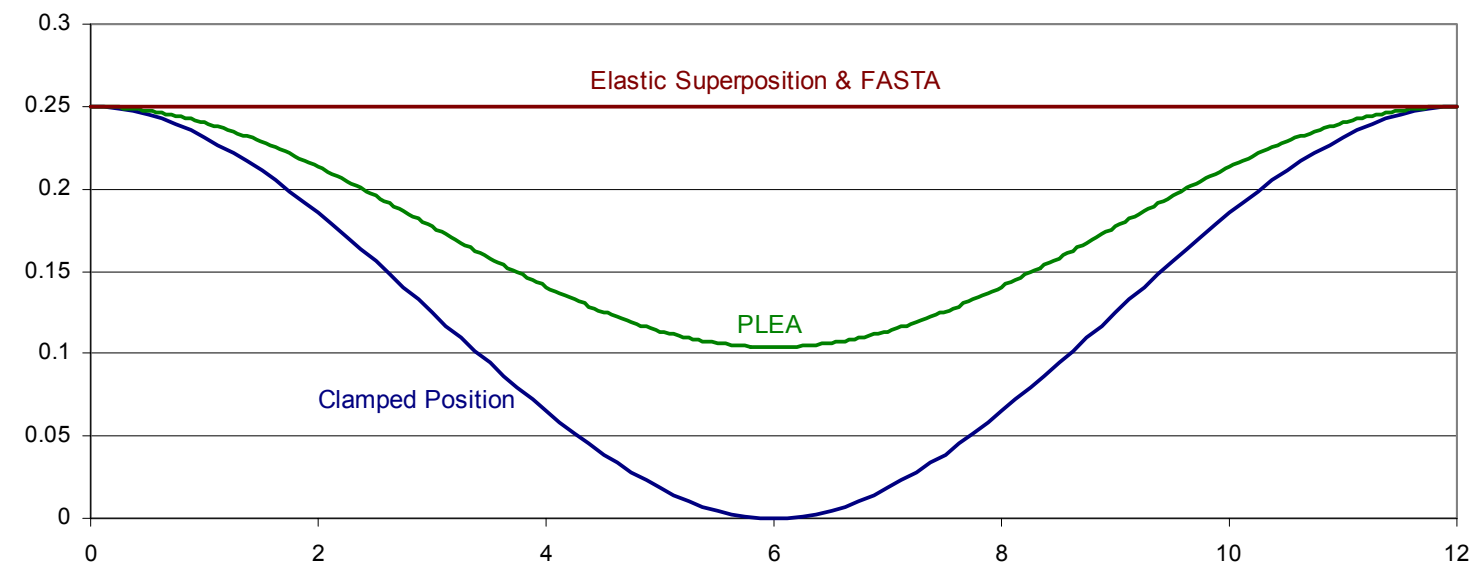

Figure 4-17: Final positions of two beam analysis, equal length, equal offset

The results of PLEA analysis demonstrate that axial forces prevent the assembly from returning to its original offset, which elastic superposition was unable to predict. 


\subsubsection{Two Beam Assembly, Unequal Length, Equal Offset}

Another assembly easily compared is one in which the beams have the same properties and offsets from the fixture, but are differing lengths. This analysis will yield both force and moment reactions, and is identical to the analysis in Section 4.2.4.2.

Table 4.5: Beam properties for unequal length, equal offset analysis

\begin{tabular}{|l|l|l|l|l|}
\hline & E & I & Beam Length & Offset $(\delta)$ \\
\hline Beam 1 & $2.9 \times 10^{7}$ psi & $4.167 \times 10^{-5}$ in $^{4}$ & 4 in & 0.25 in \\
\hline Beam 2 & $2.9 \times 10^{7}$ psi & $4.167 \times 10^{-5}$ in $^{4}$ & 8 in & 0.25 in \\
\hline
\end{tabular}

The graph below shows subtle differences between the results obtained from PLEA (green) and those obtained from elastic superposition (brown). The clamped position is also graphed in blue.

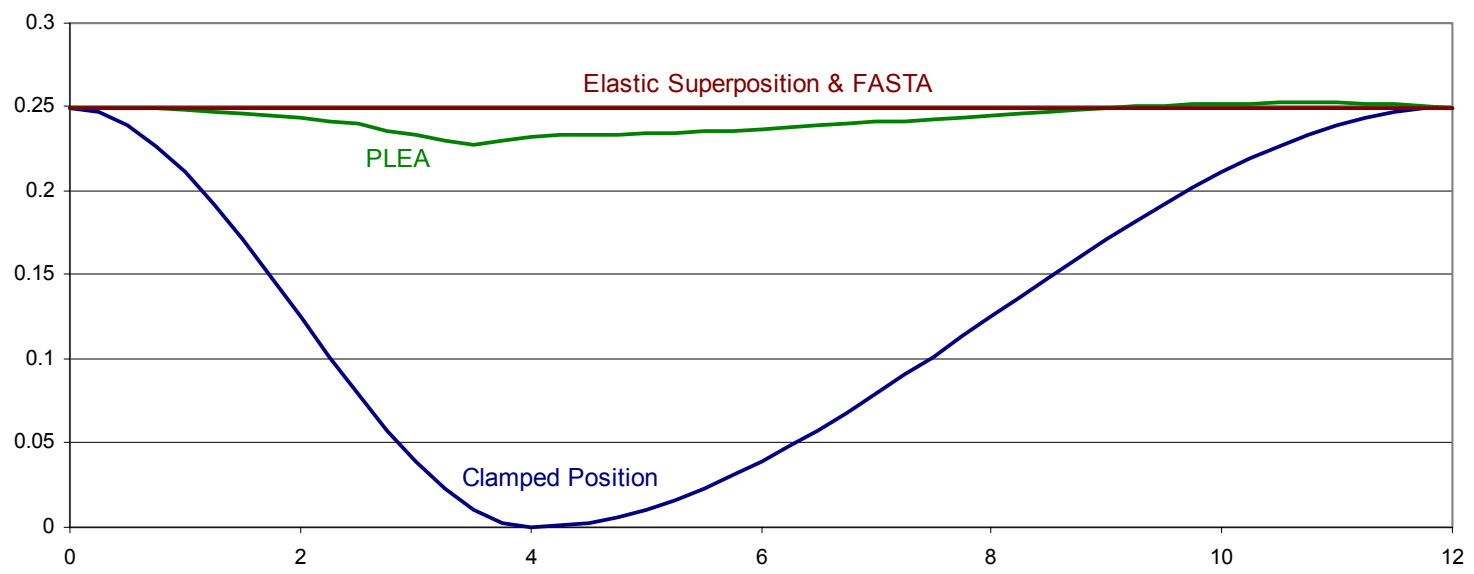

Figure 4-18: Final positions two beam assembly, unequal length, equal offset 


\subsubsection{Two Beam Assembly, Equal Length, Unequal Offset}

The final assembly for comparison is one in which the beams have the same properties and lengths, but exhibit different offsets from the fixture. This analysis will yield both force and moment reactions. The analysis was setup as listed in Table 4.6.

Table 4.6: Beam properties for equal length, unequal offset analysis

\begin{tabular}{|l|l|l|l|l|}
\hline & E & I & Beam Length & Offset $(\delta)$ \\
\hline Beam 1 & $2.9 \times 10^{7} \mathrm{psi}$ & $4.167 \times 10^{-5} \mathrm{in}^{4}$ & 6 in & 0.4 in \\
\hline Beam 2 & $2.9 \times 10^{7} \mathrm{psi}$ & $4.167 \times 10^{-5} \mathrm{in}^{4}$ & 6 in & 0.2 in \\
\hline
\end{tabular}

The graph below shows great differences between the results obtained from PLEA (green) and those obtained from elastic superposition (brown). The final position found by elastic superposition will always occur between the beams, at a ratio dependent upon each beam's stiffness, as stated before. This result is identical to the result obtained by FASTA, where the final position after assembly is obtained solely by force equilibrium.

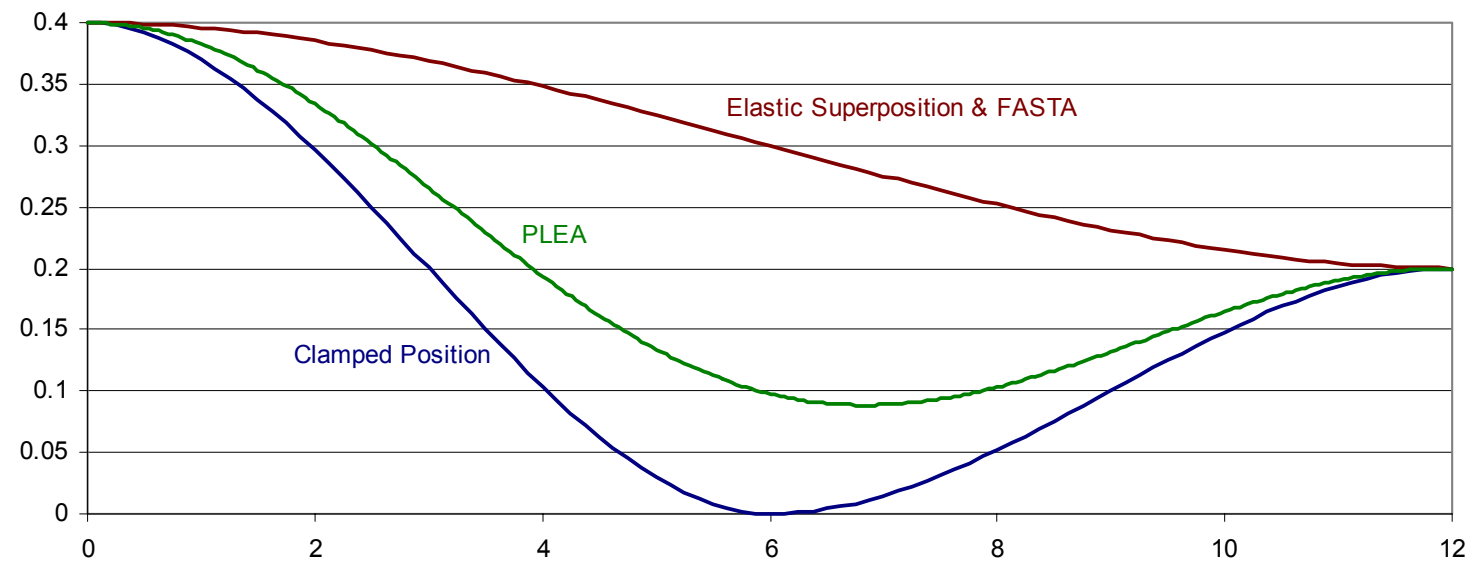

Figure 4-19: Final Positions of two beam assembly, equal length, unequal offset 


\subsubsection{Piecewise-Linear Elastic Analysis Comparisons}

To further demonstrate axial stiffness effects, 21 loading cases were analyzed. This test was performed using force-and-moment closure on two identical beams, each offset a distance $\delta$ from a fixture, as shown on Figure 4-20 and similar to the analysis in Section 4.3.3.1. As the gap distance $\delta$ was increased, the superposition springback (from elastic superposition) was compared to the springback predicted by PLEA (as determined by ANSYS $^{\circledR}$ ). These results are graphed in non-dimensional form below.

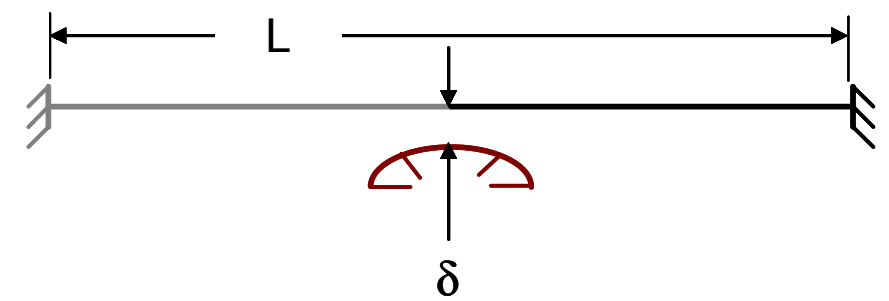

Figure 4-20: Loading test case

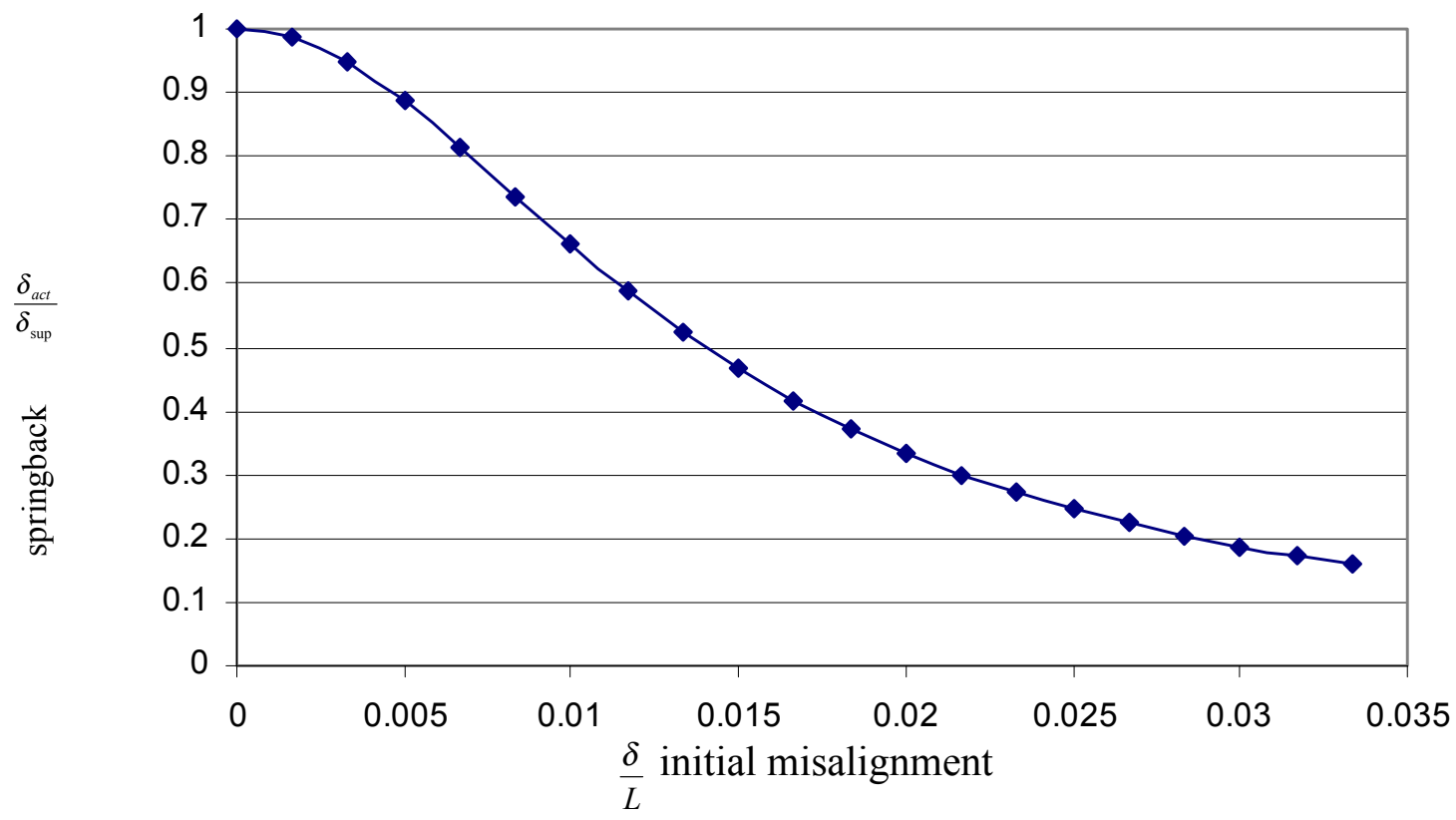

Figure 4-21: Ratio of PLEA springback to elastic superposition springback with increasing gap 
From Figure 4-21, it is apparent that as the gap distance increases, the actual springback decreases, due to the increasing prominence of axial forces. Additionally, since

tolerances can range to $1 / 100$ times the actual part dimensions or smaller, a $\delta / L$ ratio of 0.01 can occur. This means that just within the range of common tolerances, a 35\% error may occur if elastic superposition alone were used. Figure 4-21 is graphed in nondimensional form, and is only dependent upon geometric configuration, not on material properties. Thus, using elastic superposition, we will always expect errors according the error curve.

\subsection{Conclusion}

Two assembly analysis methods were introduced, elastic superposition, which analyzes the stiffness of the assembly as if the parts are in their undeformed positions, and piecewise-linear elastic analysis, PLEA, which calculates a new stiffness of the assembly when the components are in their deformed positions and also includes axial stiffness to in-plane loads. Both assembly methods were demonstrated using simple examples involving the assembly of two beams.

The results of the two methods were graphed and compared in three examples. Elastic superposition was shown to yield results independent of fixture position, akin to the results obtained by FASTA. PLEA, however, accounted for fixture position by accounting for the deformed positions of the components and in-plane stiffness. 
To compare the two results with increasing gap from the fixture, an experiment was run and the results graphed. The graph showed the increasing influence of axial forces with increasing gap distance, leading to error even in the common ranges of tolerances. This relation is accounted for in PLEA, not elastic superposition. A PLEA process map will be laid out in the next chapter, similar to the FASTA process maps laid out by Mortensen [2002]. PLEA is further examined in analysis of a more complex assembly and verified on real part data in Chapters 6 and 7. 


\section{Chapter 5: Piecewise-Linear Elastic Assembly Process}

In this chapter, the PLEA process is generalized to assemblies with multiple closure points. The process is described analytically by matrix equations relating the assembly forces and deflections. The statistical covariant solution is derived for characterizing the principle sources of variation in production processes. The PLEA process is also laid out in process maps, according to established PCFR (position, clamp, fasten, release) conventions. These maps will help the designer to determine the inputs, intermediate steps, and outputs of each step in the PLEA process. They will also serve as an aid for the software developer. This chapter assumes the reader has had some exposure to the FASTA process as laid out by Mortensen [2002], who showed how dimensional, geometric and surface variations can be combined, through statistical covariance, with elastic deformation analysis to predict variation in compliant assemblies.

An overview of the analysis process is given in Figure 5-1. The four colored sections shown simulate the PCFR assembly process commonly employed in industry. 


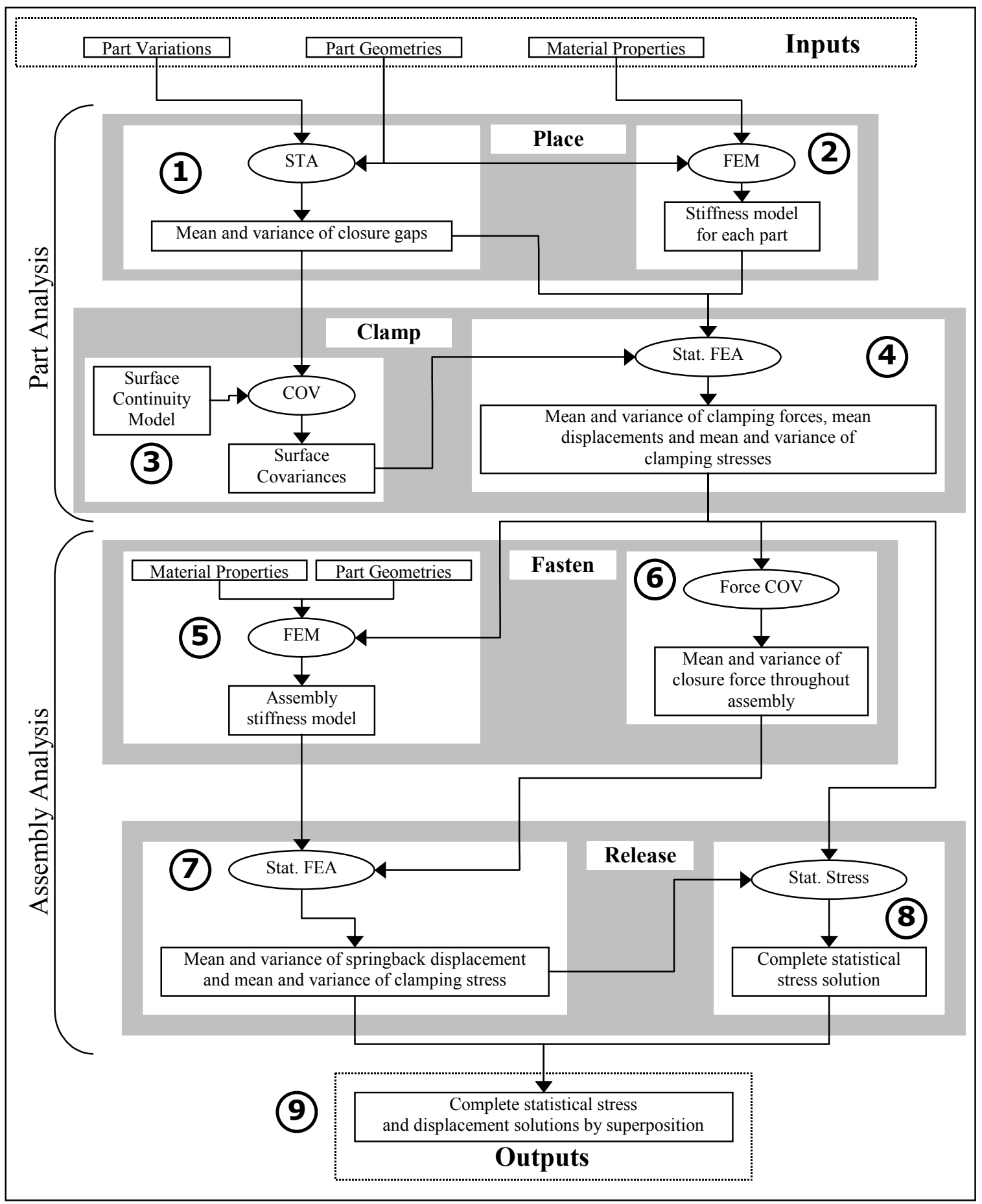

Figure 5-1: PLEA process flow diagram

The following sections give detailed descriptions of the PLEA analysis process for simulating PCFR assembly processes. Each section in Figure 5-1 will be illustrated in 
detail in the following chapter. A minor naming convention has been adopted to distinguish similar terms and prevent confusion. If the rank of the stiffness matrix is reduced to boundary nodes alone, a red (for reduced) subscript will be placed within the matrix brackets. The first matrix or vector subscript after the brackets defines the entity ( $j$ for part $j$, asm for the assembly) to which it is applied. The second subscript refers to additional information needed to distinguish the term to which it is applied. For instance, $c$ refers to a complete solution when it is necessary to specify, while $s$ refers to the springback-stage analysis, $c l$ refers to clamping-stage analysis or components in the clamped position, and $f$ refers to the final solution to the assembly analysis. Table 5.1 and Table 5.2 define the symbols which will be used throughout this chapter. 
Table 5.1: Table of symbols used in the placement and clamping stages

\section{Placement}

1. Determine Component Misalignments

$\left\{\mu_{\delta}\right\}_{j} \quad$ Mean gap between closure node and closure point on fixture of part $j$

$\left\{\sigma_{T}^{2}\right\}_{j} \quad$ Translational variation vector of part $j$

$\left\{\sigma_{R}^{2}\right\}_{j} \quad$ Rotational variation vector of part $j$

$\left\{\sigma_{S}^{2}\right\}_{j} \quad$ Surface variation vector of part $j$

2. FEM of Compliant Parts

$[\mathbf{K}]_{\mathrm{j}} \quad$ Complete stiffness matrix of part $j$

$\left[\mathbf{K}_{\text {red }}\right]_{\mathrm{j}} \quad$ Reduced stiffness matrix of part $j$

\section{Clamping}

3. Covariance Calculations

$[S]_{j} \quad$ Sensitivity matrix of part $j$ derived from surface continuity conditions

$\left[\Sigma_{G}\right]_{j} \quad$ Geometric covariance of part $j$

$\left[\Sigma_{\text {Trans }}\right]_{j} \quad$ Rigid-body translational covariance of part $j$

$\left[\Sigma_{R o t}\right]_{j} \quad$ Rigid-body rotational covariance of part $j$

$\left[\Sigma_{\delta}\right]_{j} \quad$ Overall gap covariance of part $j$

4. Statistical FEA Solution for the Clamping Analysis

$\left\{\mu_{F}\right\}_{j} \quad$ Mean clamping force of part $j$

$\left[\Sigma_{F}\right]_{j} \quad$ Clamping force covariance of part $j$

$\left\{\mu_{\delta}\right\}_{j, c} \quad$ Complete mean displacement of part $j$

$\left[\Sigma_{\delta}\right]_{j, c} \quad$ Complete displacement covariance of $\operatorname{part} j$

$[D]_{j} \quad$ Global constitutive matrix of part $j$

$[B]_{j} \quad$ Global kinematic matrix of part $j$

$\left\{\mu_{\sigma}\right\}_{j} \quad$ Complete mean nodal stress solution of part $j$ due to clamping

$\left[\Sigma_{\sigma}\right]_{j}^{T} \quad$ Complete nodal stress covariance solution of part $j$ due to clamping 
Table 5.2: Table of symbols used in the fastening and release stages

\section{Fastening}

5. FEM of the Clamped Assembly

$[\mathbf{K}]_{\text {asm }}$ Complete stiffness matrix of the assembly in its clamped position

$\left[\mathbf{K}_{\text {red }}\right]_{\text {asm }}$ Reduced stiffness matrix of the assembly in its clamped position

6. Statistical Springback Force Calculations

$\left\{\mu_{F}\right\}_{a s m, s} \quad$ Mean springback force for the assembly

$\left[\Sigma_{F}\right]_{a s m, s} \quad$ Springback force covariance matrix for the assembly

\section{Release}

7. Statistical FEA Solution for the Springback Analysis

$\left\{\mu_{\delta}\right\}_{a s m, f} \quad$ Complete mean springback displacement for the assembly

$\left[\Sigma_{\delta}\right]_{a s m, f} \quad$ Complete springback displacement covariance for the assembly

$[D]_{a s m} \quad$ Global constitutive matrix for the assembly

$[B]_{a s m} \quad$ Global kinematic matrix for the assembly

$\left\{\mu_{\sigma}\right\}_{a s m, s} \quad$ Complete mean nodal stress solution of the assembly due to springback

$\left[\Sigma_{\sigma}\right]_{a s m, s} \begin{gathered}\text { Complete nodal stress covariance solution of the assembly } \\ \text { due to springback }\end{gathered}$

8. Overall Statistical Stress Solution

$\left\{\mu_{\sigma}\right\} \quad$ Overall mean nodal stress solution

$\left[\Sigma_{\sigma}\right]$ Overall nodal stress covariance solution 


\subsection{Component Placement}

In the analysis of the placement stage of the assembly, it is assumed the parts are located on the fixture and are ready to be clamped and assembled. Two steps must be accomplished in the component placement stage to characterize the components. First, the gaps between the closure points and the fixture, which occur as a result of the accumulation of dimensional and process variations, must be estimated statistically. Second, the stiffness relationship of each component based upon their mean geometries must be obtained through FEM.

\subsubsection{Step 1 -Determine Component Misalignments}

Step 1 of the PLEA process involves determining the mean and variance of the misalignment in the parts with the fixture. This can be achieved by modeling the misalignment using statistical analysis. Alternately, the component misalignment can be determined between the components and the fixture when actual production components are placed in their fixtures and physically measured. One of the fundamental differences between the FASTA method and the PLEA method is the FASTA predicts the final state of the assembly, independent of the fixturing employed. In PLEA, assembly gaps can occur for each component in the assembly, generally as the gap between the component and the rigid fixture. For example, in a two-component assembly, the FASTA method would consider the closure of one gap between the two mating surfaces, while the PLEA method would consider the closure of the two gaps occurring between each component and the fixture. 
Statistical tolerance analysis can be employed to estimate the misalignment strictly due to dimensional and fixture variations. A set of vector loops is constructed to model the gaps between closure points on a component and corresponding closure points on the fixture. By analyzing the mean dimensions, the mean gap $\left\{\mu_{\delta}\right\}$ is determined for each designated closure point $i$ on the component $j$. By statistically analyzing the tolerance stackup in the loops, the contribution of component and fixture variations to the translational variation $\left\{\sigma_{T}^{2}\right\}$, the rotational variation $\left\{\sigma_{R}^{2}\right\}$, and the surface variation $\left\{\sigma_{S}^{2}\right\}$ at the gap are determined at each designated closure point $i$ along the assembly surface. Figure 5-2 illustrates the variation contributions as they apply to total mating surface variation. The entire population of component $j$ is characterized by the mean and the covariance of the gap.

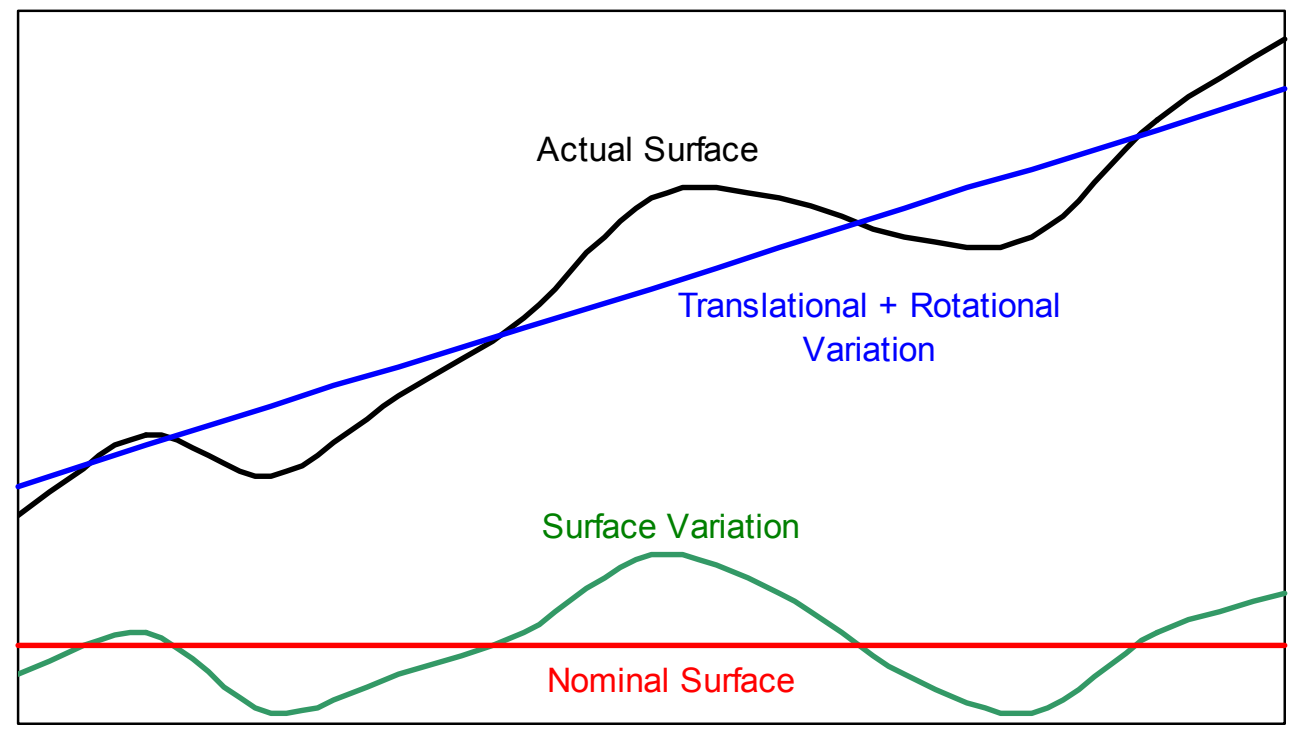

Figure 5-2: Variation contribution example

It is important to separate the gap into these three variation components. Translational and rotational rigid-body variations constitute the major "air gap" between the nominal part and the mean of the actual parts, and are analyzed independent of surface variation. 
After the rigid body "air gaps" are calculated, surface variation constitutes the remaining error in the part, which can be simulated by adding surface variance vectors at each closure point. Knowledge of the translational, rotational and surface variation contributions to the assembly variation can be used to improve product quality by identifying the chief variation contributions and seeking corresponding process improvements.

In FASTA, five terms are determined from the assembly analysis: the mean gap $\left\{\mu_{\delta}\right\}$, the translational variation $\left\{\sigma_{T}^{2}\right\}$, the rotational variation $\left\{\sigma_{R}^{2}\right\}$, and the surface variations for parts $\mathrm{A}$ and $\mathrm{B},\left\{\sigma_{S A}^{2}\right\}$ and $\left\{\sigma_{S B}^{2}\right\}$. If the assembly is analyzed as a rigid body, only the translational and rotational rigid-body variations would be needed to characterize the gap of the entire population of assemblies. Surface variation is necessary to describe the surface error on flexible surfaces, but only those pertaining directly to the gap surface. Generally, the surface error describes surface waviness. Small-scale effects, such as surface roughness, can be neglected. Large-scale effects due to warping can be included in the rotational variation, as demonstrated in Mortensen [2002].

The PLEA process differs somewhat from FASTA, because the compliant parts are first positioned and clamped on a rigid fixture, independent of each other. To describe the variation in a single clamping process, only four terms are obtained: the mean gap $\left\{\mu_{\delta}\right\}$, the translational variation $\left\{\sigma_{T}^{2}\right\}$, the rotational variation $\left\{\sigma_{R}^{2}\right\}$, and the surface variation for the compliant part, $\left\{\sigma_{S}^{2}\right\}$. This process must then be repeated for each flexible part $j$ 
brought in contact with the fixture. If variation information is not available from measurements of actual production parts, these quantities may be estimated by tolerance and surface waviness callouts. Table 5.3 summarizes the steps for determining misalignments. Figure 5-3 diagrams the process and the inputs and outputs for each step.

Table 5.3: Summary of steps to determine misalignments

Inputs: part geometries, part variations, fixture geometries

Outputs: variation components $\left\{\sigma_{T}^{2}\right\}_{j},\left\{\sigma_{R}^{2}\right\}_{j},\left\{\sigma_{S}^{2}\right\}_{j}$ and mean $\left\{\mu_{\delta}\right\}_{j}$ of gaps

Analysis Steps:

A. Variation Component Solutions

Translational Variation

1. Create vector loop model for assembly component and the fixture

2. Write kinematic vector loop equation

3. Take partial derivatives of vector loop equations

4. Populate DLM matrix and vector

5. Apply statistical stack-up model to open loop equation

6. Calculate variance at each fastening point

7. Create translational variance vector

Rotational Variation

1. Calculate rotational variation of mating gap part

2. Create the rotational variance vector

Surface Variation

1. Identify tolerance bands at each flexible gap surface

2. Calculate surface variance

3. Create the surface variance vector

B. Mean Gap Solution

1. Solve for mean gap value at each fastening point using:

a. Vector loop equations and nominal dimensions

b. CAD assembly model

2. Output mean gap vector

C. Repeat for each compliant part $j$ 


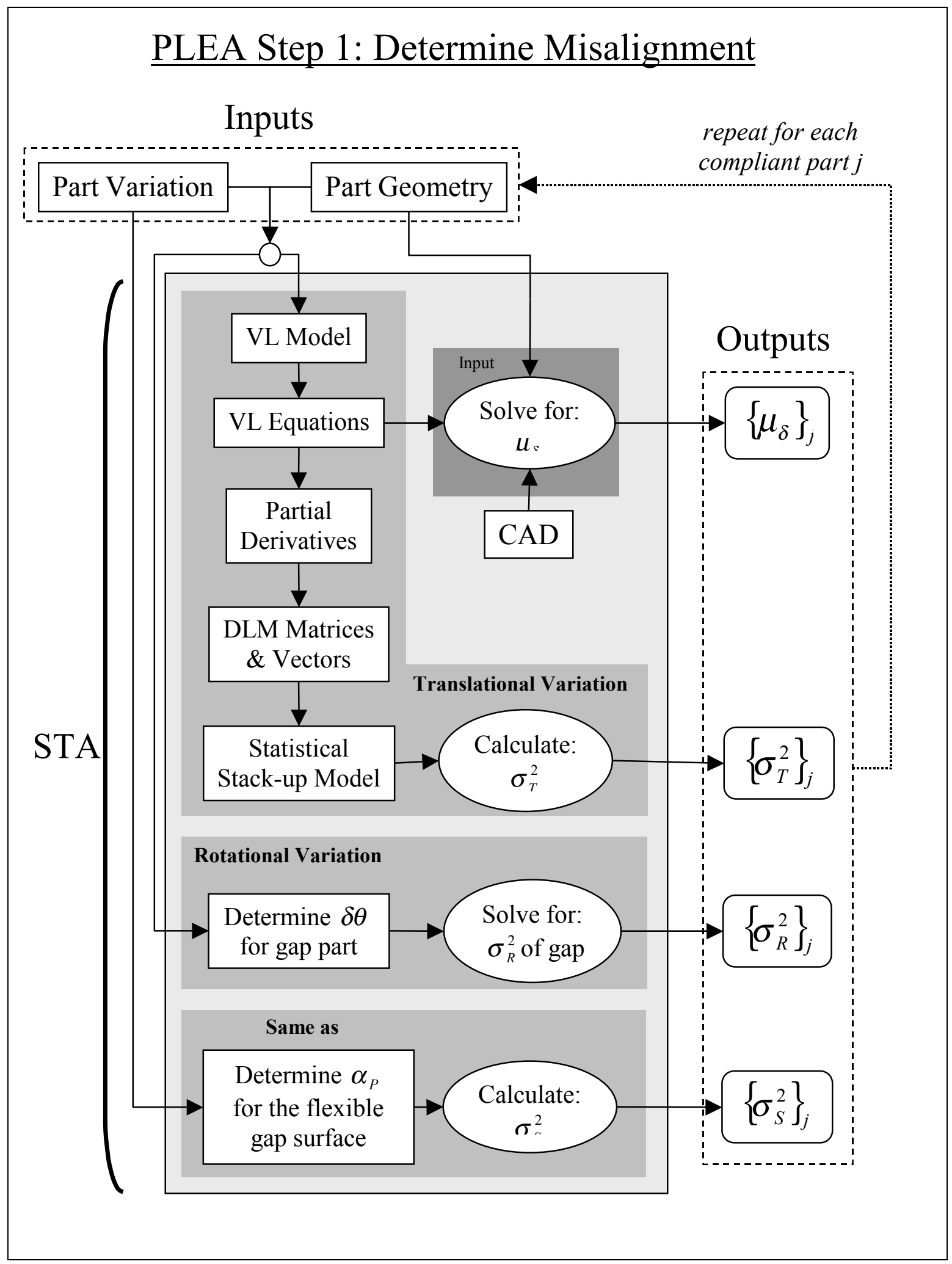

Figure 5-3: Process flow diagram for statistically determining misalignment 


\subsubsection{Step 2 - Finite Element Modeling of Compliant Parts}

In compliant assemblies, it is necessary to include a finite element model of the assembly and its component parts in order to determine the force-to-deflection relationship. This relationship is necessary in relating the closure gaps to the assembly forces. A stiffness matrix $[\mathbf{K}]$ is derived for each component, based upon the component's mean geometry. These stiffnesses are subsequently used to obtain a statistical FEA solution to characterize variation in compliant assembly processes. Additionally, the stiffness matrix can be substructured into the reduced stiffness matrix $\left[\mathbf{K}_{\text {red }}\right]$, to reduce complexity.

In the PLEA method, finite element modeling of the components is done in much the same way as the FASTA method. The geometries and material properties of each component $j$ are input into a FEA program, like ANSYS ${ }^{\circledR}$. An element type is selected and the model is meshed. The stiffness matrix $[\mathbf{K}]$ of the component $j$ is determined by the FEA program and can be output for further process steps.

When working with complex parts, often it is desirable to reduce the complexity of the analysis, thereby reducing the calculation time for the analysis. To facilitate this, the stiffness matrix can be reduced by substructuring as introduced in Section 3.3.1. The process of determining the stiffness and substructuring the matrix is repeated for each component $j$ in the assembly.

Because PLEA analyzes displacement-closed assemblies in their clamping stages rather than force-equilibrium constrained assemblies as FASTA does, the equilibrium stiffness 
matrix, $\left[\mathbf{K}_{\mathrm{eq}}\right]$, is not calculated. Table 5.4 summarizes the steps for FEM the compliant parts prior to clamping. Figure 5-4 diagrams the process and shows the desired inputs and outputs.

Table 5.4: Summary of steps to FEM compliant parts

\begin{tabular}{|l|}
\hline Inputs: part geometries, material properties \\
Output: $\left[\mathbf{K}_{\text {red }}\right]$ \\
\hline Analysis Steps: \\
\hline \\
A. Model compliant part with FEM \\
1. Define nominal geometry \\
2. Input material properties \\
3. Select element type and options \\
4. Mesh model with nodes at fastening points \\
5. Solve for stiffness matrix \\
B. Reduce stiffness matrices \\
6. Select boundary nodes and degrees of freedom to include \\
7. Calculate reduced stiffness matrix $\left[\mathbf{K}_{\mathrm{red}}\right]$ \\
C. Repeat for each compliant part $j$ \\
\end{tabular}




\section{$\underline{\text { PLEA Step 2: FEM of Compliant Parts }}$}

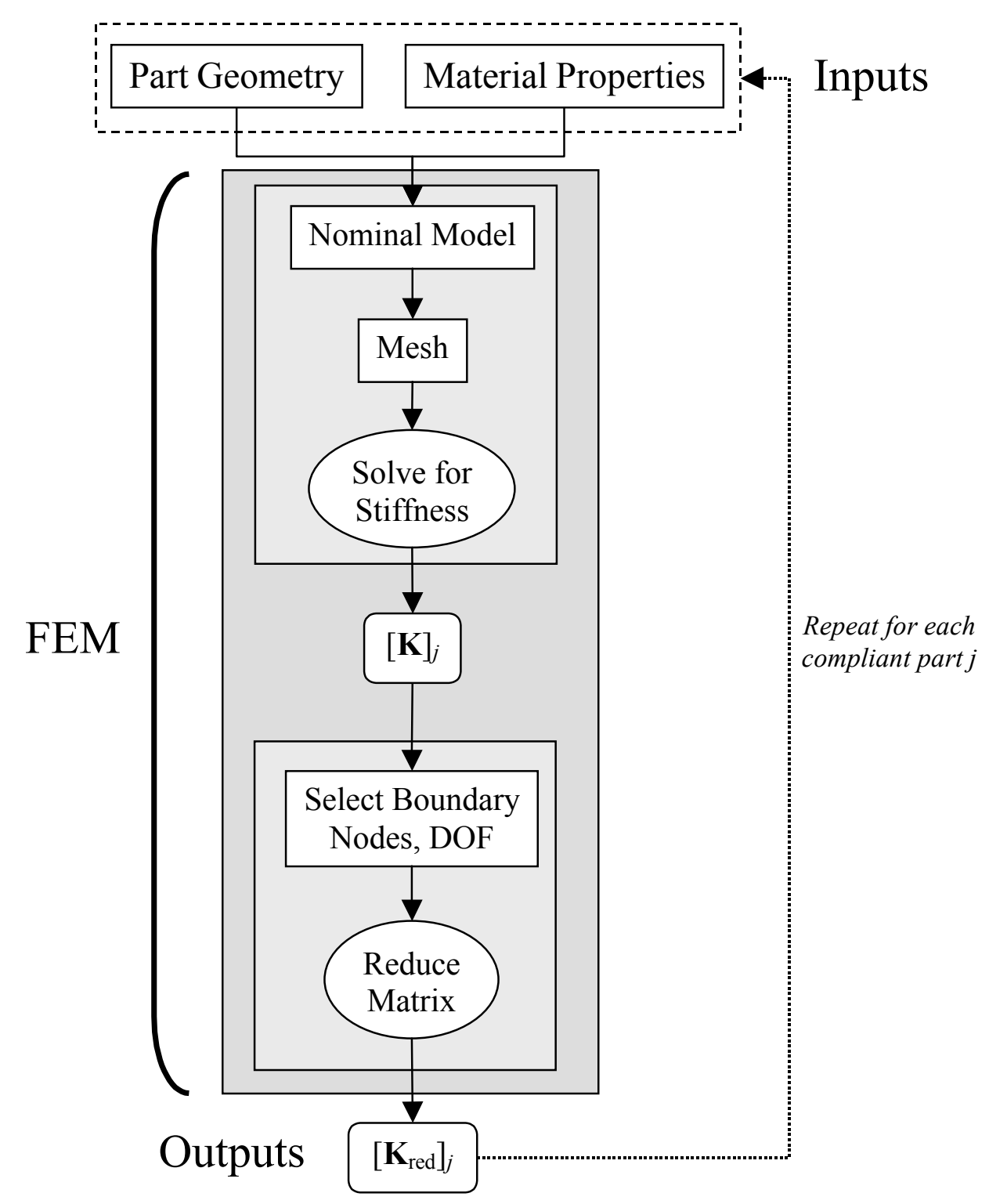

Figure 5-4: Process flow diagram for FEM of compliant parts 


\subsection{Component Clamping}

With the components and the fixture analyzed for their assembly gap characteristics and modeled in FEA, we can analyze the clamping stage of the assembly. To do this, we first need to derive the gap covariance for each component and the fixture. After the gap covariances are determined, the clamping analysis may take place through statistical FEA.

\subsubsection{Step 3 - Covariance Calculations}

In this step of the PLEA process, the covariance of each gap is determined. This process is similar to that of FASTA, with minor differences. Because there is one gap per part, there exists one gap covariance per part. This gap covariance can be solved independently for each part $j$. To perform this operation, first the surface variation component of part $j\left\{\sigma_{S}^{2}\right\}_{j}$ is combined with a surface continuity model to estimate the geometric covariance for part $j$ according to Equation 5-1.

$$
\left[\Sigma_{G}\right]_{j}=[S]_{j}\left[\Sigma_{S}\right]_{j}[S]_{j}^{T}
$$

where $[S]_{j}$ is the sensitivity matrix derived from surface continuity conditions and the wavelength spectrum of each component $j$. The values of $\left\{\sigma_{S}^{2}\right\}_{j}$ populate the diagonal of matrix $\left[\Sigma_{S}\right]_{j}$.

The rigid-body translational and rotational covariances are calculated according to Equations 5-2 and 5-3. 


$$
\begin{array}{rlr}
{\left[\Sigma_{\text {Trans }}\right]_{j}} & =\left[S(0)_{\mathrm{lg}}\right]_{j}\left[\Sigma_{T}\right]_{j}\left[S(0)_{\mathrm{lg}}\right]_{j}^{T} & \text { Eq. 5-2 } \\
{\left[\Sigma_{\text {Rot }}\right]_{j}} & =\left[S(1)_{\mathrm{lg}}\right]_{j}\left[\Sigma_{R}\right]_{j}\left[S(1)_{\mathrm{lg}}\right]_{j}^{T} & \text { Eq. 5-3 }
\end{array}
$$

where $\left[S(0)_{\mathrm{lg}}\right\rfloor_{j}$ and $\left[S(1)_{\mathrm{gg}}\right\rfloor_{j}$ are sensitivity matrices associated with the rigid body

modes of dimensional variation. The values of $\left\{\sigma_{T i}^{2}\right\}_{j}$ and $\left\{\sigma_{R i}^{2}\right\}_{j}$ populate the diagonal

of matrices $\left[\Sigma_{T}\right]_{j}$ and $\left[\Sigma_{R}\right]_{j}$ respectively.

Once these covariances are found, the overall gap covariance for part $j$ is found according to Equation 5-4.

$$
\left[\Sigma_{\delta}\right]_{j}=\left[\Sigma_{G}\right]_{j}+\left[\Sigma_{\text {Trans }}\right]_{j}+\left[\Sigma_{\text {Rot }}\right]_{j}
$$

Eq. 5-4

The process to find the gap covariance is then repeated for each part $j$ in the assembly.

Table 5.5 summarizes the steps for calculating the gap covariances and Figure 5-5

diagrams the procedure.

Because the gaps are formed only when the parts are placed in their fixtures, additional sources of error can be introduced during the placement stage, not associated with the previous shape of the part. These error sources can be both repeatable and random. These error sources might include:

- Misalignment with the fixture due to locating error

- Operator error when positioning in the fixture

- Surface variations at the constrained end of the part 
It is vital to note that these error effects are accounted for in mean shifts and rigid-body variations. Both mean shifts and translational and rotational rigid-body effects can be used in diagnosing error sources. Other effects could be included in the gap covariance calculation, such as operator error when clamping, if they could be measured and characterized statistically. It is anticipated with experience and metrology, the error sources can be diagnosed and all non-random sources of error can be identified and eliminated.

Table 5.5: Summary of steps to calculate covariances of the gaps

Inputs: $\left\{\sigma_{T}^{2}\right\}_{j},\left\{\sigma_{R}^{2}\right\}_{j},\left\{\sigma_{S}^{2}\right\}_{j}$

Outputs: $\left[\Sigma_{\delta}\right]_{j}$

Analysis Steps:

A. Covariance Component Solutions

I. Geometric covariance of flexible surface

1. Create transformation matrix using surface continuity model

2. Create diagonal matrix using surface variation variance vector

3. Calculate geometric covariance for surface

II. Translational Covariance (Rigid)

1. Create transformation matrix using zero-order Legendre polynomial

2. Create diagonal matrix using translational variance vector

3. Calculate translational covariance of gap

III. Rotational Covariance (Rigid)

1. Create transformation matrix using first-order Legendre polynomial

2. Create diagonal matrix using rotational variance vector

3. Calculate rotational covariance of gap

B. Gap Covariance Calculation

1. Sum geometric, translational, and rotational covariance components

2. Output solution

C. Repeat for each part $j$ 


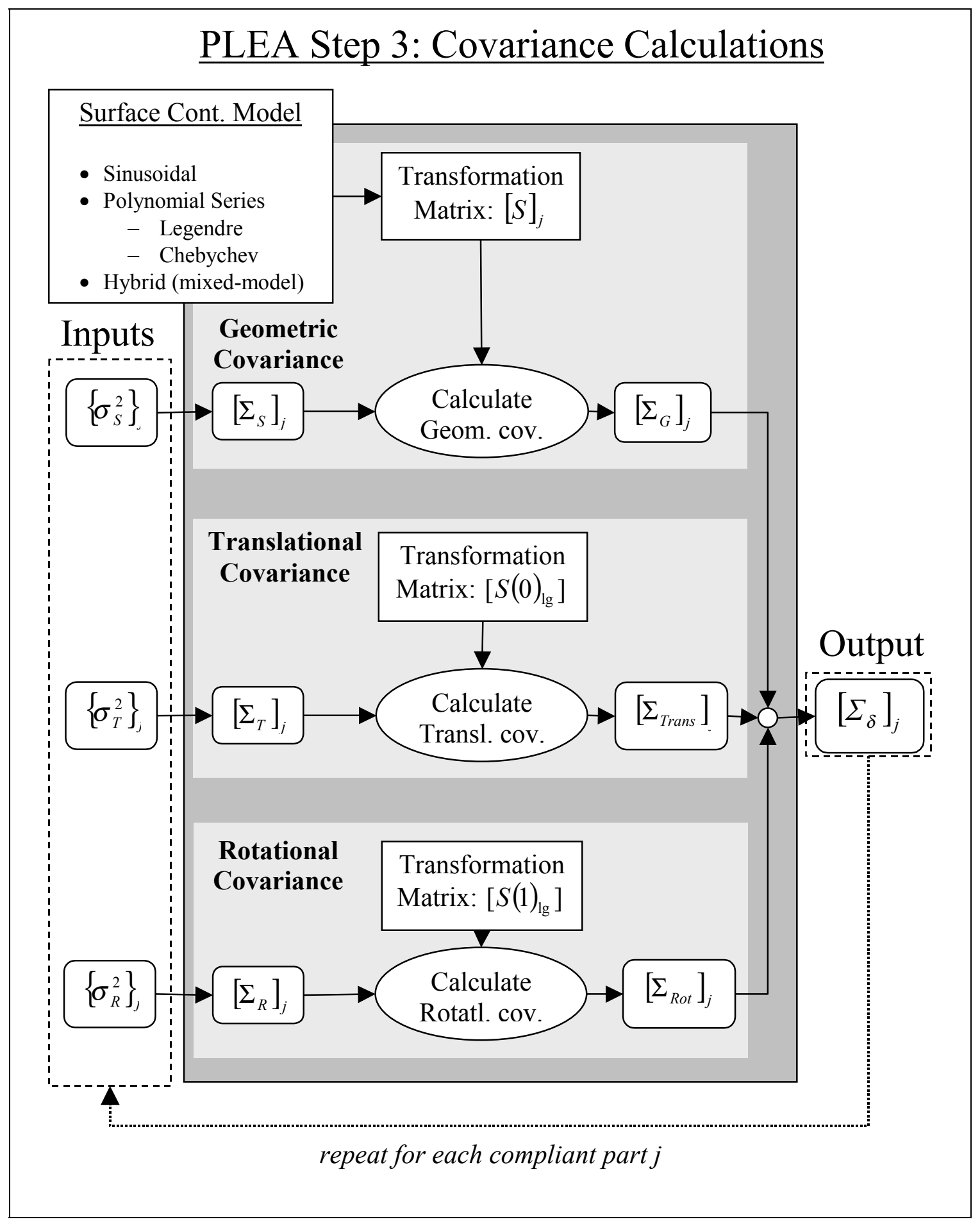

Figure 5-5: Process flow diagram for calculating covariances of the gaps 


\subsubsection{Step 4 - Statistical FEA Solution for the Clamping Analysis}

The statistical FEA solution for the clamping of each component to the fixture involves applying sets of loads and boundary conditions, which simulate the clamping process to the mean geometric stiffness of each part. With the quantities for stiffness, mean gap and gap covariance determined in previous steps for each part $j$, the complete statistical FEA solution for each part due to clamping can be determined.

Because PLEA relies upon the superposition of linear analyses to simulate a nonlinear analysis, the statistical FEA solution for the clamping stage must first be obtained and added to the statistical FEA solution for the springback stage, which will be calculated in later steps. With the mean gap vectors, the gap covariance matrices and the reduced stiffness matrices of each component $j$ found in the previous analysis steps, we are able to statistically calculate clamping force, stress and deformation variations. These analyses can be performed independently, since the clamping analysis of one component has unique values, independent of all other components.

The clamping stage assembly forces and overall deformation in each part $j$ occur as the part is assembled to the fixture. These quantities are found by first solving Equations 5-5 and 5-6 for the mean and covariance of the clamping forces for each part $j$, with the mean gaps, the covariances of the gaps, and the reduced stiffness matrices determined in previous steps as inputs.

$$
\begin{array}{cc}
\left\{\mu_{F}\right\}_{j}=\left[K_{\text {red }}\right]_{j}\left\{\mu_{\delta}\right\}_{j} & \text { Eq. 5-5 } \\
{\left[\Sigma_{F}\right]_{j}=\left[K_{\text {red }}\right]_{j}\left[\Sigma_{\delta}\right]_{j}\left[K_{r e d}\right]_{j}^{T}} & \text { Eq. 5-6 }
\end{array}
$$


With the statistical clamping forces calculated, the statistical deflections can be calculated throughout each part $j$. This is done by applying the previously-solved assembly forces and boundary conditions to the unreduced stiffness matrix for part $j$, according to Equations 5-7 and 5-8.

$$
\begin{array}{cc}
\left\{\mu_{F}\right\}_{j}=[K]_{j}\left\{\mu_{\delta}\right\}_{j, c} & \text { Eq. 5-7 } \\
{\left[\Sigma_{F}\right]_{j}=[K]_{j}\left[\Sigma_{\delta}\right]_{j, c}[K]_{j}^{T}} & \text { Eq. 5-8 }
\end{array}
$$

Solving Equations 5-7 and 5-8 for $\left\{\mu_{\delta}\right\}_{j, c}$ and $\left[\Sigma_{\delta}\right]_{j, c}$ yield the complete statistical nodal deflections of each node of component $j$, not only the deflections on the boundary nodes. These nodal deflections are used to model the new, assembled and deformed components as well as determine the mean and covariance of the stress due to clamping. This clamping stress solution can be determined by Equations 5-9 and 5-10.

$$
\begin{gathered}
\left\{\mu_{\sigma}\right\}_{j}=[D]_{j}[B]_{j}\left\{\mu_{\delta}\right\}_{j, c} \\
{\left[\Sigma_{\sigma}\right]_{j}=[D]_{j}[B]_{j}\left[\Sigma_{\delta}\right]_{j, c}[B]_{j}^{T}[D]_{j}^{T}}
\end{gathered}
$$

PLEA uses the mean shape of the parts for stiffness matrix computation. With the complete solution of the mean clamping displacement $\left\{\mu_{\delta}\right\}_{j, c}$ the mean shape of the assembly can be determined for subsequent analysis steps. It is assumed in all instances that all variant shapes of the parts are likewise clamped into the mean clamping shape. Because of this, the statistical quality of the population is stored in clamping force covariance terms $\left[\Sigma_{F}\right]_{j}$ with the solution of Equation 5-8. It is assumed in this thesis that initial misalignment in the placement stage is the only source of variation. The displacement covariance $\left[\Sigma_{\delta}\right]_{j, c}$ is useful in determining the stress covariance due to clamping, but is not used in subsequent assembly analysis steps. 
The necessary outputs of the clamping analysis statistical FEA solution are the means and covariances of the assembly forces for each component being clamped, the mean deflections throughout each component being clamped, and the means and the covariances of the stress solutions throughout each component in its clamped position. These values are stored for further analysis. The deflected nodes become the starting configuration in the next step in the FEA. Table 5.6 summarizes the FEA solution for the clamping process and Figure 5-6 illustrates the process.

As mentioned in Section 3.5.2, the stresses are determined in conventional FEA elementby-element for a single structural member by applying nodal displacements obtained from the global solution to a single element. However, for statistical FEA, each element can be affected by every other element throughout the covariance matrix, as demonstrated in Bihlmaier [1998]. It is therefore necessary to build the global constitutive $[D]$ and kinematic $[B]$ matrices for the entire model in order to see the influence of the off-diagonal terms in the stress covariance. Hopefully, this task can be automated in commercial applications. 
Table 5.6: Summary of statistical FEA solution for the clamping analysis

Inputs: $\left\{\mu_{\delta}\right\}_{j},\left[\Sigma_{\delta}\right]_{j},\left[K_{r e d}\right]_{j},[K]_{j}$

Outputs: $\left\{\mu_{F}\right\}_{j},\left[\Sigma_{F}\right]_{j},\left\{\mu_{\delta}\right\}_{j, c},\left\{\mu_{\sigma}\right\}_{j},\left[\Sigma_{\sigma}\right]_{j}$

Analysis Steps:

A. Statistical Solutions of Clamping Forces

1. Instantiate reduced stiffness matrix

2. Calculate mean clamping force

3. Calculate clamping force covariance matrix

4. Repeat for each component $j$ in assembly

B. Statistical Solutions for Complete Part Clamping Displacements

1. Obtain complete stiffness matrix from FEA

2. Apply clamping forces and boundary conditions as loads in FEA

3. Solve for complete mean nodal displacement

4. Apply force covariances and boundary conditions as loads in FEA

5. Solve for complete covariance of nodal displacement

6. Repeat for each component $j$ in assembly

C. Statistical Solutions for Part Clamping Stresses

1. Obtain global constitutive and kinematic matrices from FEA

2. Apply complete mean nodal deformation vector

3. Solve for mean nodal stress

4. Apply complete nodal deformation covariance matrix

5. Solve for nodal stress covariance

6. Repeat for each component $j$ in assembly 


\section{PLEA Step 4: Statistical Solution for Clamping Analysis}

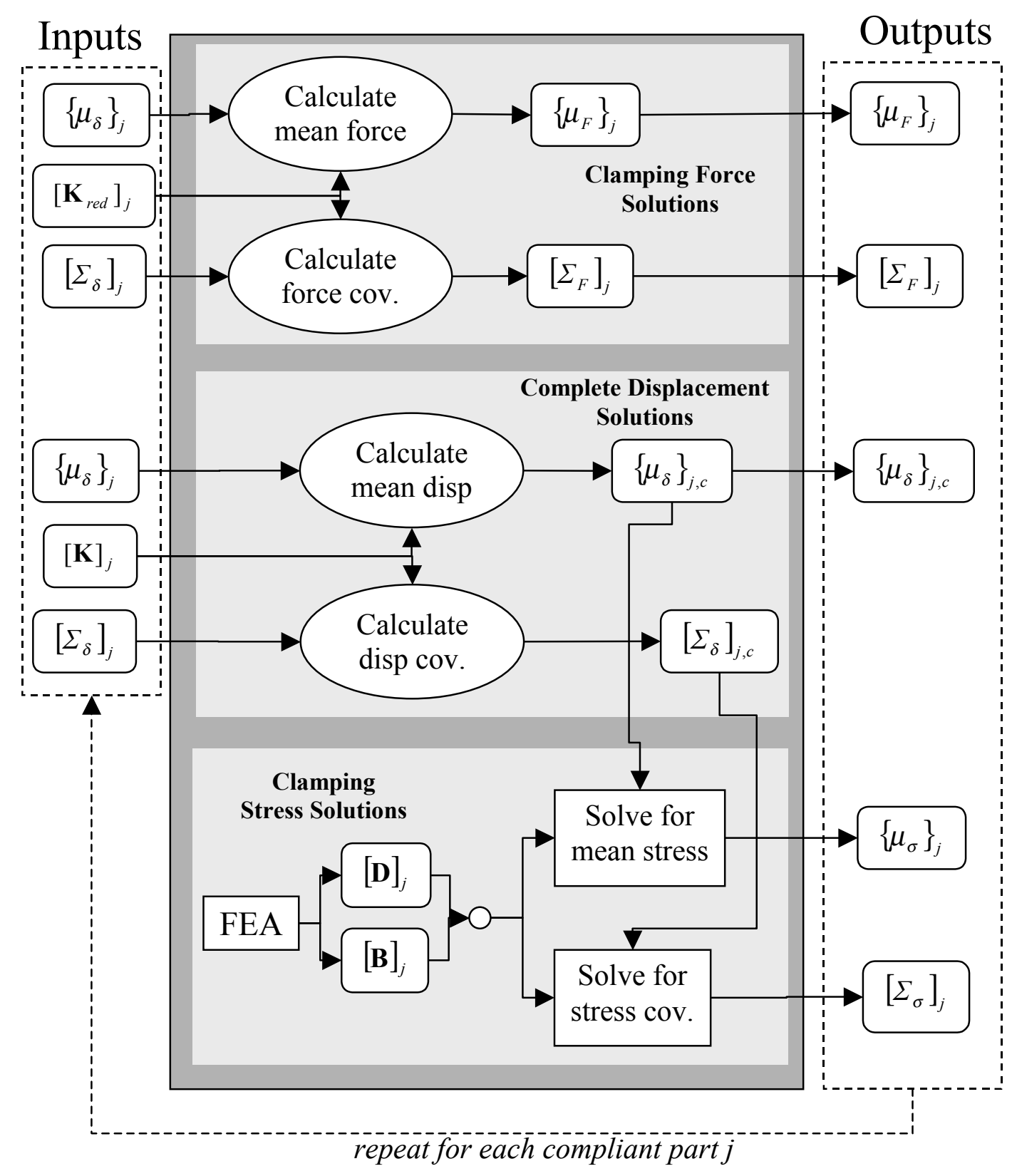

Figure 5-6: Process flow diagram for statistical FEA solution of the clamping analysis 


\subsection{Assembly Fastening}

With the part deflections and statistical clamping forces determined, the assembly fastening and springback analysis can be performed. In PLEA, the assembly fastening process transforms the outputs from the placement and clamping stages into inputs for the release stage. This is accomplished in two steps. First, based on the mean initial component geometries and the mean displacements, the stiffness of the clamped assembly is determined through FEM. Second, the statistical springback forces for the assembly are computed from the statistical clamping forces of the components.

\subsubsection{Step 5 - Finite Element Modeling of Clamped Assembly}

When the nodal deflections due to clamping have been determined, they can be added to the initial nodal coordinates to obtain a deflected component. When this is accomplished, a new global assembly stiffness matrix $[K]_{a s m}$ can be constructed from the deformed geometry. Each deflected part $j$ is modeled for FEA analysis, and meshed with the same mesh densities as the individual parts.

Note that it is this step that defines the piecewise-linear method. Calculating a modified stiffness matrix due to deformation resulting from clamping approximates nonlinear

effects of fixtured assembly processes. Additionally, it allows for "arch effects", due to axial forces acting along the centerline of beams and thin sheets, to contribute to the changes in stiffness. 
To simulate the "assembly" of the components, nodal constraints can be applied to corresponding assembly nodes of the individual deflected parts. These nodal constraints constrain the independent degrees of freedom to be equal, thus reducing the number of independent degrees of freedom. A simple example of this can be seen with the assembly of two cantilever beams, as in Figure 5-7.

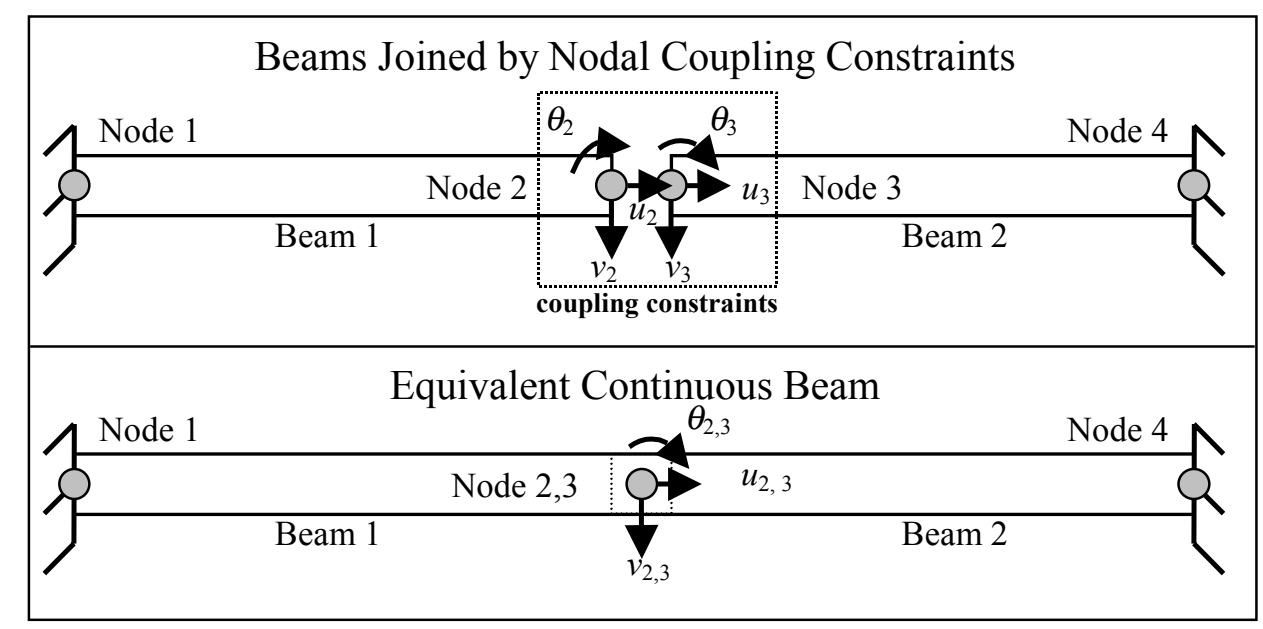

Figure 5-7: Model of a fastened assembly using nodal constraints

In the above illustration, both node 2 and node 3 exhibit three independent degrees of freedom each; translation vectors $u$ and $v$, and rotation $\theta$. Applying nodal constraints, such that $u_{2}=u_{3}, v_{2}=v_{3}$, and $\theta_{2}=\theta_{3}$, removes three independent degrees of freedom. Essentially, one node is eliminated by constraining it to move with a neighboring node. Because of these nodal constraints, beam 1 and beam 2 constitute one continuous beam, since both forces and moments can be transmitted between the beams. Modeling the assembly as one continuous piece or as multiple components with applied nodal constraints is equivalent. ANSYS ${ }^{\circledR}$ has the ability to couple nodal DOFs via the CP command, which is used in all subsequent analyses. 
The application of nodal coupling is not restricted to coincident nodes. This approach can be generalized to include nodes with small offsets held rigidly together. An example of such includes lap joints welded or riveted together. In these cases, the closure nodes have an offset equal to the thickness of the parts, but still exhibit coupling in all DOFs. This generalization was used in the analyses in Chapter 6.

With nodal constraints applied to individual parts, the global stiffness matrix $[K]_{a s m}$ can be calculated for the complete assembly. This assembly stiffness may also be substructured into a reduced assembly stiffness matrix $\left[K_{\text {red }}\right]_{a s m}$ to reduce complexity, but this reduction is not required. Table 5.7 summarizes the steps for modeling the clamped assembly for springback analysis, and Figure 5-8 diagrams the process.

Table 5.7: Summary of steps to FEM the clamped assembly

Inputs: part geometries, material properties, $\left\{\mu_{\delta}\right\}_{j, c}$

Output: $\left[\mathbf{K}_{\text {red }}\right]_{\text {asm }},[\mathbf{K}]_{\text {asm }}$

Analysis Steps:

A. Model compliant assembly with FEM

1. Input nominal nodal positions of all parts

2. Sum deflected nodal positions to obtain nominal assembly position

3. Select element type and options

4. Input material properties

5. Mesh model with nodes at fastening points

6. Apply fastening nodal constraints

7. Solve for stiffness matrix $[\mathbf{K}]_{\text {asm }}$

B. Reduce stiffness matrix

1. Select boundary nodes and degrees of freedom to include

2. Calculate reduced stiffness matrix $\left[\mathbf{K}_{\mathrm{red}}\right]_{\text {asm }}$ 


\section{PLEA Step 5: FEM of Clamped Assembly}

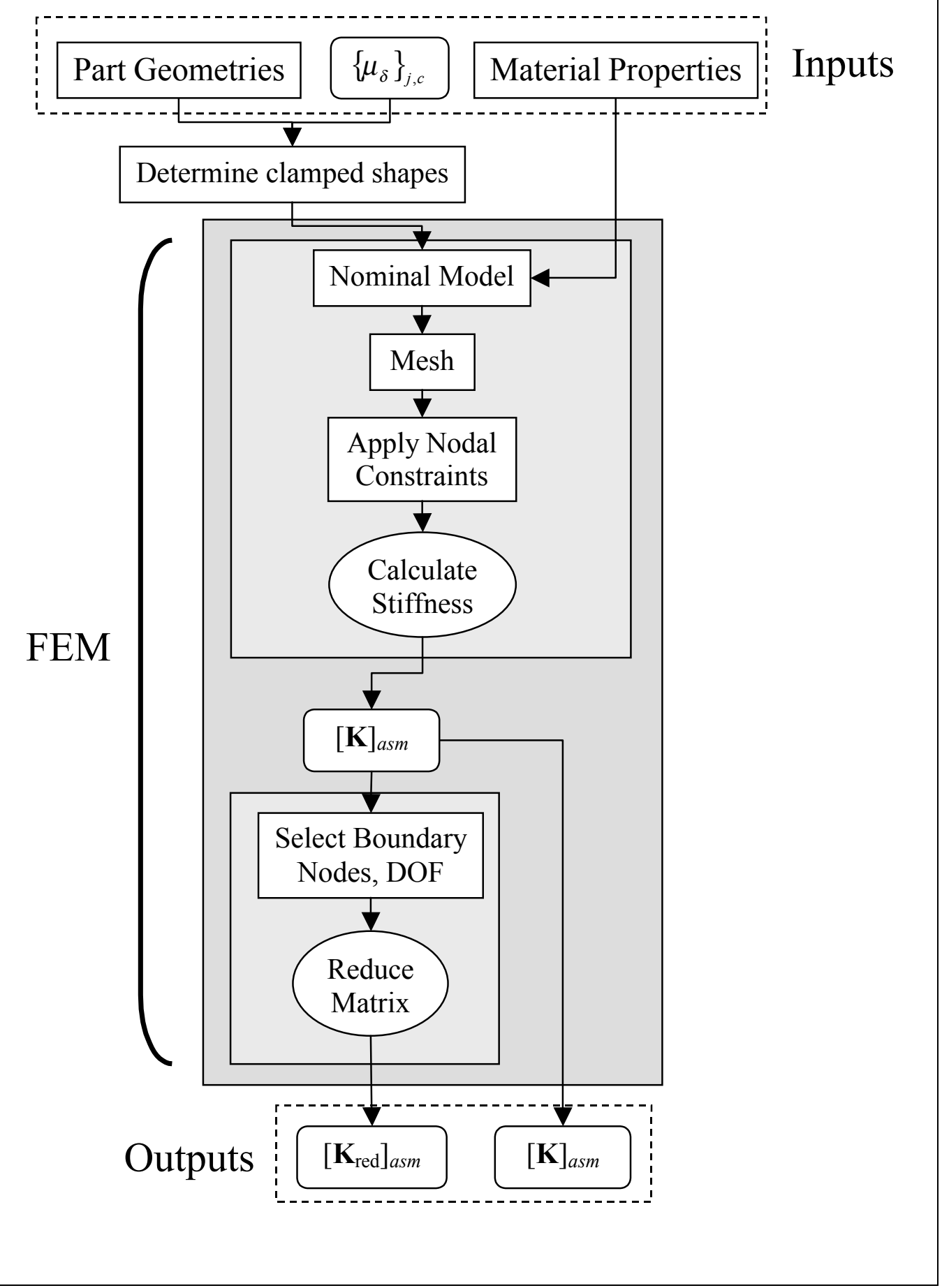

Figure 5-8: Process flow diagram for FEM of clamped assembly 


\subsubsection{Step 6-Statistical Springback Force Calculations}

In this step, the mean and variance of the springback forces are obtained. During the clamping analysis, the displacement constraints necessary to constrain each part to the fixture were determined. Using the linear force-displacement relationships given in Equations 5-5 and 5-6, these displacements were equated to clamping forces. Upon release from the fixture, the complements to these forces return each part to its original, undeformed position.

When the parts were assembled and released, these clamping force complements act upon the assembly. To determine the clamping force complements, or springback forces, the statistical clamping forces of all parts must be summed. In classical statistics, variances, which are the squares of the standard deviations, can be linearly summed if they are independent. This step provides us with the mean $\left\{\mu_{F}\right\}_{a s m, s}$ and variance $\left[\Sigma_{F}\right]_{a s m, s}$ of the springback forces for the assembly.

$$
\begin{gathered}
\left\{\mu_{F}\right\}_{a s m, s}=-\sum_{j=1}^{n}\left\{\mu_{F}\right\}_{j} \\
{\left[\Sigma_{F}\right]_{a s m, s}=\sum_{j=1}^{n}\left[\Sigma_{F}\right]_{j}}
\end{gathered}
$$

The mean springback forces are equal in magnitude to the mean closure forces. They are applied to the corresponding nodes on the assembly in the reverse direction.

It should be noted that the mean clamping force vectors and the covariant clamping force matrices obtained through statistical FEA of the parts are of smaller rank than the mean force vector and covariant force matrix of the assembly. Therefore, the part values must 
be mapped to the positions of corresponding nodes in the mean springback force vector and the covariant springback force matrix of the assembly.

The superposition of these two sets of forces results in zero external applied force on the finished assembly. There are, however, internal forces remaining which, although they are self-equilibrating, they produce residual stress internal to the assembly. Table 5.8 summarizes modifications of the FEA model for springback analysis and Figure 5-9 illustrates the process.

Table 5.8: Summary of steps to statistical springback force calculations

Inputs: $\left\{\mu_{F}\right\}_{i},\left[\Sigma_{F}\right]$

Output: $\left\{\mu_{F}\right\}_{a s m, s},\left[\Sigma_{F}\right]_{a s m, s}$

Analysis Steps:

A. Create mean springback force vector

1. Obtain all part mean assembly force vectors

2. Organize quantities for corresponding nodes

3 . Solve for mean springback force vector

B. Create springback force covariance matrix

1. Obtain all part assembly force covariance matrices

2. Organize quantities for corresponding nodes

3. Solve for springback force covariance matrix 


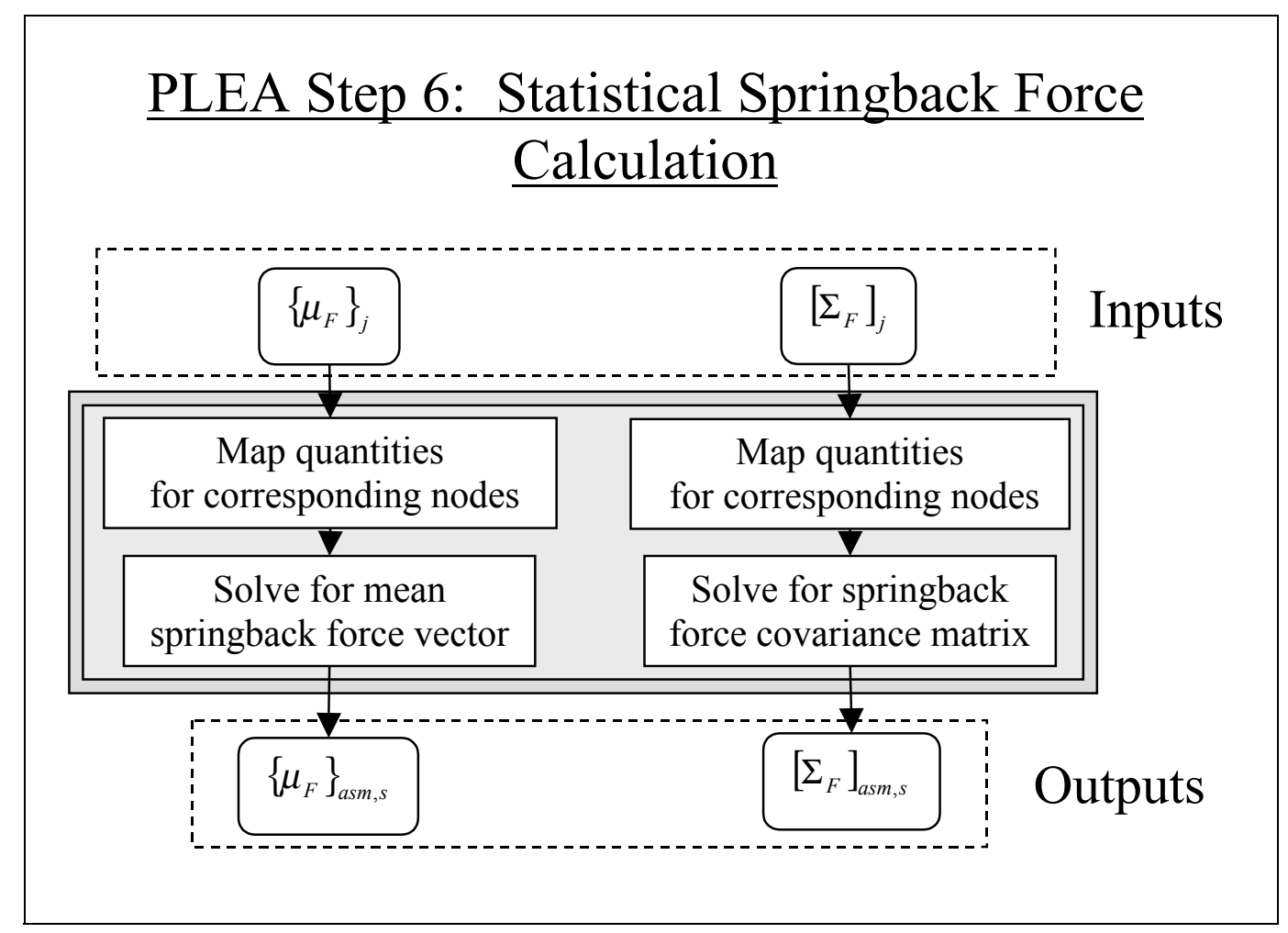

Figure 5-9: Process flow diagram for calculating the statistical springback forces

\subsection{Assembly Release}

The application of the statistical springback forces to the modified assembly constitutes the release stage. The springback forces were stored elastically in the members during clamping and are released when the clamps are removed.

\subsubsection{Step 7 -Statistical FEA Solution for the Springback Analysis}

The mean final shape of the assembly is found with the solution for $\left\{\mu_{\delta}\right\}_{a s m}$ and the variance associated with the assembly shape is found with the solution for $\left[\Sigma_{\sigma}\right]_{a s m}$. With the quantities $\left\{\mu_{F}\right\}_{a s m, s},\left[\Sigma_{F}\right]_{a s m, s}$, and $\left[K_{r e d}\right]_{a s m}$, Equations 5-13 and 5-14 can be solved. 


$$
\begin{array}{cc}
\left\{\mu_{\delta}\right\}_{a s m}=\left[K_{r e d}\right]_{a s m}^{-1}\left\{\mu_{F}\right\}_{a s m, s} & \text { Eq. 5-13 } \\
{\left[\Sigma_{\delta}\right]_{a s m}=\left[K_{\text {red }}\right]_{a s m}^{-1}\left[\Sigma_{F}\right]_{a s m, s}\left[K_{r e d}\right]_{a s m}^{T-1}} & \text { Eq. 5-14 }
\end{array}
$$

The displacements solved above represent only the displacements of the boundary nodes of the assembly. To obtain the complete statistical nodal displacements of each node of the assembly, the full assembly stiffness matrix $[K]_{a s m}$ must be used with the assembly boundary conditions and applied statistical springback forces in their fully-populated vectors.

$$
\begin{array}{cc}
\left\{\mu_{\delta}\right\}_{a s m, f}=[K]_{a s m}^{-1}\left\{\mu_{F}\right\}_{a s m, s} & \text { Eq. } \mathbf{5 - 1 5} \\
{\left[\Sigma_{\delta}\right]_{a s m, f}=[K]_{a s m}^{-1}\left[\Sigma_{F}\right]_{a s m, s}[K]_{a s m}^{T} \quad-1} & \text { Eq. } \mathbf{5 - 1 6}
\end{array}
$$

The mean and variance of the assembly stresses due to springback can also be determined according to Equations 5-17 and 5-18. Once again, these quantities are evaluated at every node in the assembly. $[D]_{a s m}$ and $[B]_{a s m}$ are the global constitutive and kinematic matrices for the complete assembly, respectively.

$$
\begin{array}{cc}
\left\{\mu_{\sigma}\right\}_{a s m, s}=[D]_{a s m}[B]_{a s m}\left\{\mu_{\delta}\right\}_{a s m, c} & \text { Eq. } \mathbf{5 - 1 7} \\
{\left[\Sigma_{\sigma}\right]_{a s m, s}=[D]_{a s m}[B]_{a s m}\left[\Sigma_{\delta}\right]_{a s m, c}[B]_{a s m}^{T}[D]_{a s m}^{T}} & \text { Eq. 5-18 }
\end{array}
$$

As experience accumulates, it may be determined how to circumvent the full assembly modeling. This can be done by determining how far apart two elements can be before covariant effects can be neglected. It may be that one can return to individual part models to determine interior stress and deflection results rather than relying upon the global assembly model. 
Table 5.9 summarizes the steps to determine the statistical solution for the springback step and Figure 5-10 illustrates the process.

Table 5.9: Summary of statistical FEA solution for the springback analysis

\begin{tabular}{l} 
Inputs: $\left\{\mu_{F}\right\}_{a s m, s},\left[\Sigma_{F}\right]_{a s m, s},\left[K_{\text {red }}\right]_{a s m}$ \\
Outputs: $\left\{\mu_{\delta}\right\}_{a s m},\left[\Sigma_{\delta}\right]_{a s m},\left\{\mu_{\sigma}\right\}_{a s m, s},\left[\Sigma_{\sigma}\right]_{a s m, s}$ \\
\hline Analysis Steps: \\
A. Statistical Solutions of Springback Deflections \\
1. Obtain complete assembly stiffness matrix from FEA \\
2. Calculate mean springback deflection \\
3. Calculate springback deflection covariance matrix \\
B. Statistical Solutions for Complete Assembly Springback Deflections \\
1. Obtain complete assembly stiffness matrix from FEA \\
2. Apply springback deflections and boundary conditions as loads in FEA \\
3. Solve for mean nodal displacement (final mean shape) \\
4. Apply deflection covariances and boundary conditions as loads in FEA \\
5. Solve for covariance of nodal displacement (covariance of final shape) \\
C. Statistical Solutions for Assembly Springback Stresses \\
1. Obtain global constitutive and kinematic matrices from FEA \\
2. Apply complete mean nodal deformation vector \\
3. Solve for mean nodal stress due to springback \\
4. Apply complete nodal deformation covariance matrix \\
5. Solve for nodal stress covariance due to springback
\end{tabular}




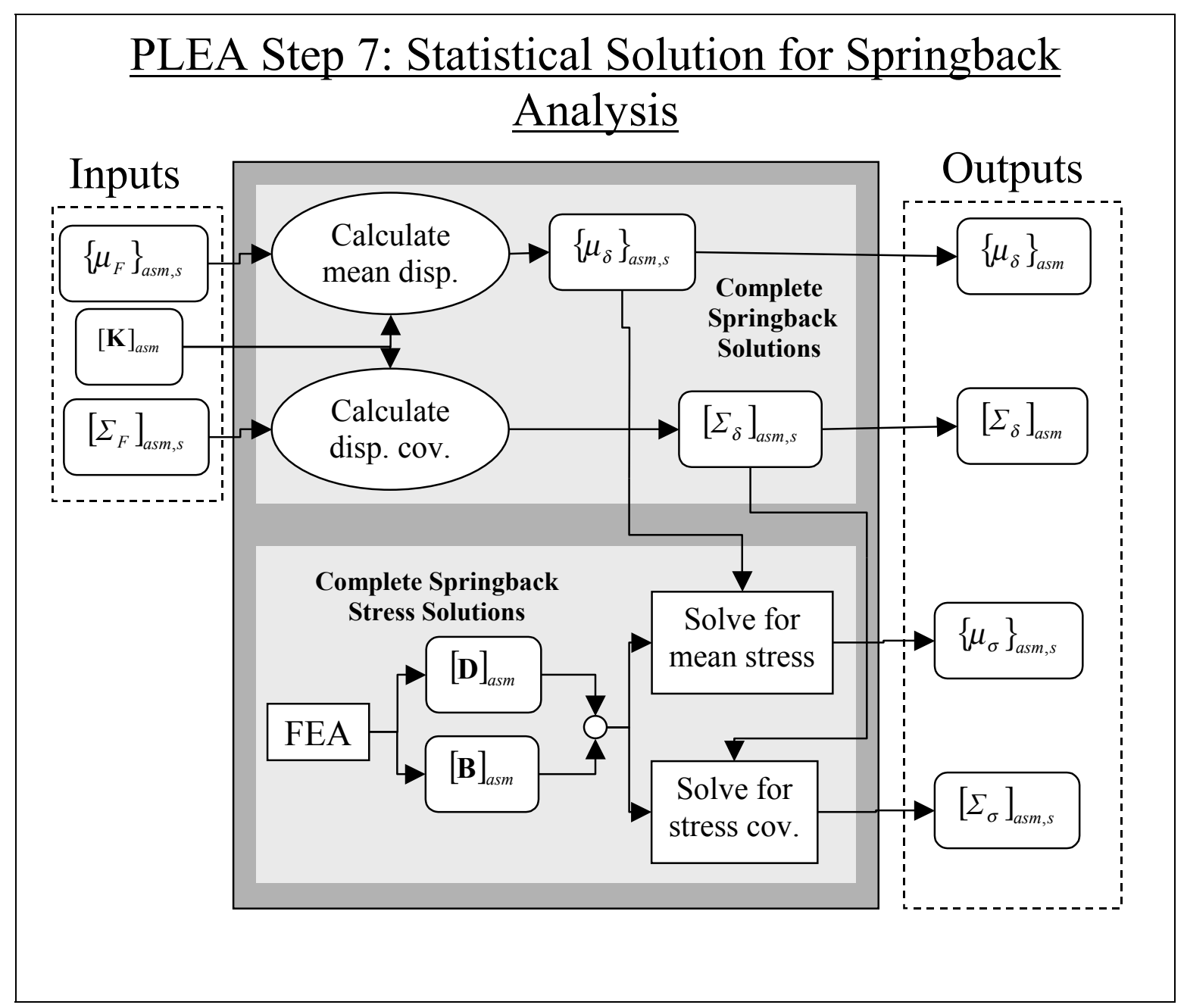

Figure 5-10: Process flow diagram for statistical FEA solution for the springback analysis

\subsubsection{Step 8-Overall Statistical Stress Solution Derivation}

The PLEA process is completed by the superposition of the results found through the clamping and springback analysis stages. The final stresses of the assembly are the result of combining the stresses due to clamping and the stresses due to springback, according to Equations 5-19 and 5-20. These must be solved node-by-corresponding node.

$$
\left\{\mu_{\sigma}\right\}_{a s m}=\left\{\mu_{\sigma}\right\}_{a s m, s}+\sum_{j=1}^{n}\left\{\mu_{\sigma}\right\}_{j}
$$




$$
\left[\Sigma_{\sigma}\right]_{a s m}=\left[\Sigma_{\sigma}\right]_{a s m, s}+\sum_{j=1}^{n}\left[\Sigma_{\sigma}\right]_{j}
$$

Because stress is generated independently in all assembly steps, net stress due to assembly is expected. Likewise, because the stiffness was recalculated for the springback analysis, net deflection due to assembly is expected. However, because the applied clamping forces and the applied springback forces are complementary, force equilibrium is achieved and there is no net force at the end of this assembly process. Table 5.10 summarizes the final steps needed to obtain the complete statistical stress solution.

Figure 5-11 illustrates the process.

Table 5.10: Summary of steps for overall statistical FEA stress solution derivation

Inputs: $\left\{\mu_{\sigma}\right\}_{a s m, s},\left\{\mu_{\sigma}\right\}_{j},\left[\Sigma_{\sigma}\right]_{a s m, s},\left[\Sigma_{\sigma}\right]_{j}$

Outputs: Final $\operatorname{Stress}(\mu, \Sigma)$

Analysis Steps:

A. Overall Mean Stress Solution

1. Obtain complete mean springback stress solution

2. Obtain complete part clamping stress solutions for all parts

3. Map vectors into corresponding nodal positions

4. Sum vectors and obtain overall nodal mean stress solution

B. Overall Stress Covariance Solution

1. Obtain complete springback stress covariance solution

2. Obtain complete part clamping stress covariance solutions for all parts

3. Map matrices into corresponding nodal positions

4. Sum matrices and obtain overall nodal stress covariance solution 


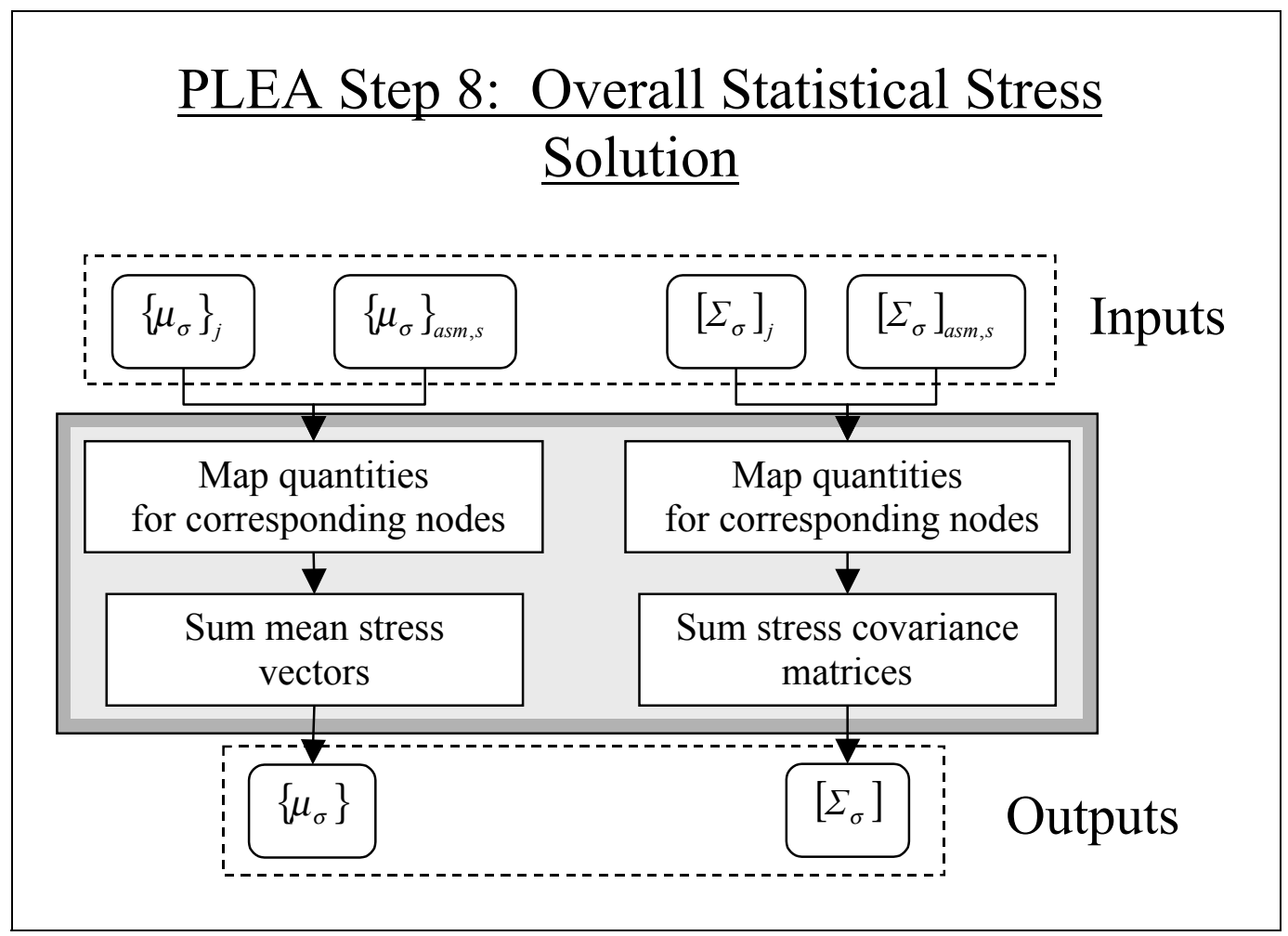

Figure 5-11: Process flow diagram for overall statistical stress solution derivation

\subsection{PLEA Process Summary}

The solution to the PLEA analysis is the statistical final shape of the assembly, as well as the statistical stress solution at each node in the assembly. Through the superposition of both the clamping and springback solutions, this is accomplished at various stages of PLEA. For the final shape of the assembly, it is accomplished with the superposition of the statistical displacement solutions to the initial geometry, according to Equations 5-21 and 5-22.

$$
\begin{aligned}
& \text { final mean geometry }=\text { initial mean geometry }+\left\{\mu_{\delta}\right\}_{c l}+\left\{\mu_{\delta}\right\}_{s p} \quad \text { Eq. 5-21 } \\
& \text { final geometry covariance }=\left[\Sigma_{\delta}\right]_{s p} \quad \text { Eq. 5-22 }
\end{aligned}
$$


These shape solutions are found when the of the deflection solution of the springback analysis is calculated. This is because the initial shape of the springback analysis is the initial mean geometry in the clamped position, or the first two terms on the right side of Equation 5-21. The shape covariance is determined with the calculation of the covariant displacement matrix in the springback stage only. This is because the only variance introduced in the assembly is from the misalignment of the parts with the fixture. The covariance of the clamping force fully describes the statistical quality of this misalignment, and is used to compute the covariant displacement matrix in the springback stage. These final shape solutions are obtained in step 7 of PLEA.

The stress solutions are likewise found using superposition. Assuming the parts are unstressed in the placement stage, the statistical stresses imparted to the assembly is simply the superimposing of the statistical stress solutions from the clamping and springback stages, according to Equations 5-23 and 5-24. The final stress solutions are obtained as illustrated in step 8 of PLEA.

$$
\begin{gathered}
\text { final mean stress }=\left\{\mu_{\sigma}\right\}_{c l}+\left\{\mu_{\sigma}\right\}_{s p} \\
\text { final stress covariance }=\left[\Sigma_{\sigma}\right]_{c l}+\left[\Sigma_{\sigma}\right]_{s p}
\end{gathered}
$$

Eq. 5-24

Because the vectors and matrices of the parts in the clamping stage are of a smaller rank than those of the assembly in the springback stage, the quantities from the clamping analysis in the above equations must be mapped to vectors and matrices of equal rank as the springback analysis, as indicated in Section 5.3.2. 
A detailed PLEA process flow diagram is given in Figure 5-12. Grayed boxes show each step of the PCFR process. The PLEA process laid out in this chapter is shown in numbered boxes. The inputs and outputs of the PLEA process are shown symbolically. Vectors and matrices reduced in rank to the boundary or closure nodes only are in rectangular boxes, while full-rank vectors and matrices are in rounded boxes. This figure gives additional detail of input and output data requirements, which will prove valuable for future work. 


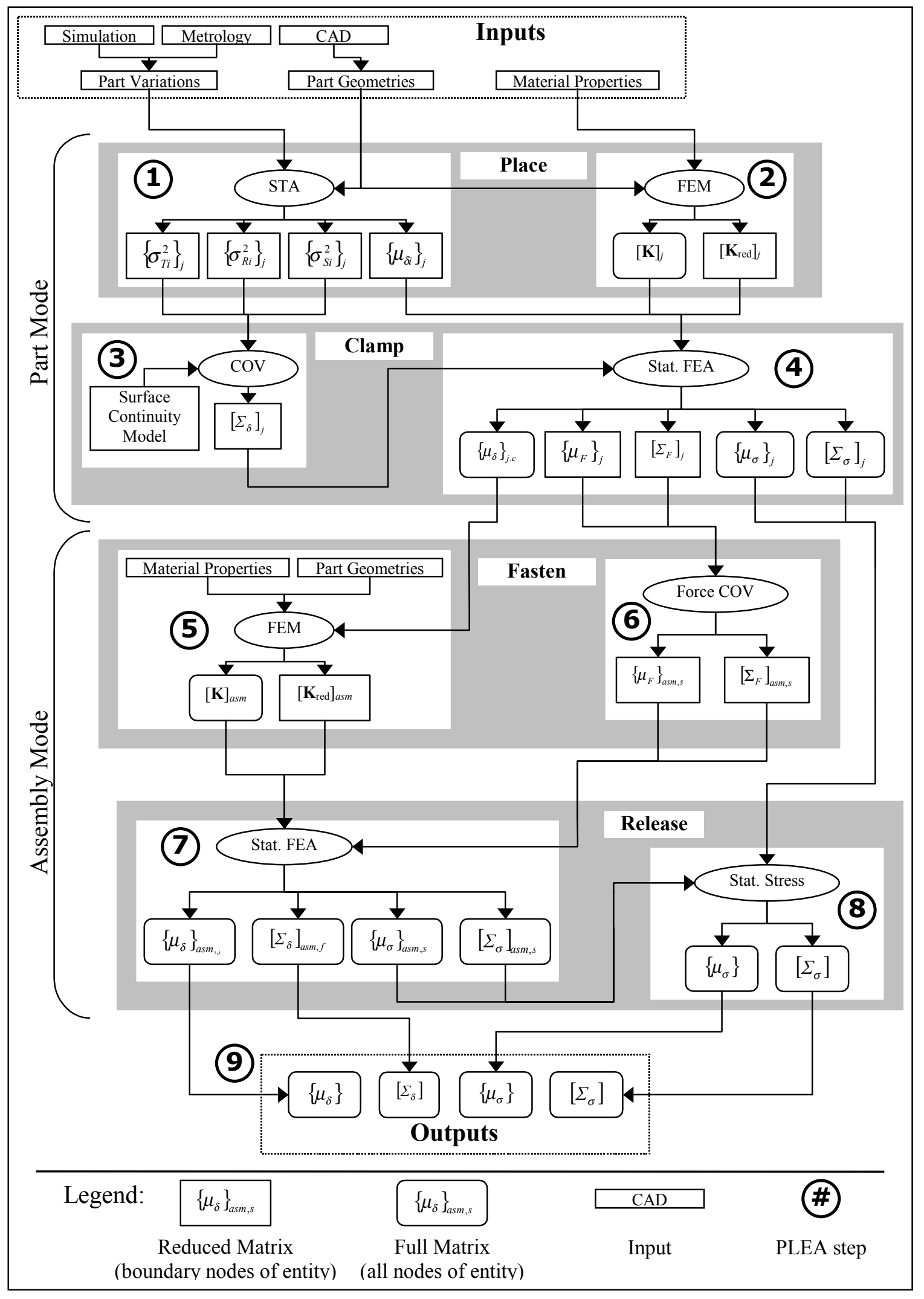

Figure 5-12: Detailed PLEA process flow diagram 


\subsection{Alternate Analysis Steps for Complex Assemblies}

The steps laid out in this chapter are valid for the analysis of a wide range of fixtured assemblies. PLEA first analyzes the assembly in part mode, where the statistical stress solution due to placement and clamping is found for each part independently. PLEA is completed in assembly mode, where the statistical stress solution of the entire assembly due to fastening and release, with subsequent springback, is calculated. These modes are valid for the analysis of all assemblies, regardless of complexity.

Sometimes, however, it is not desirable to perform calculations on the entire assembly, especially when the assembly consists of very large or multiple parts and the memory required for computation is limited. If such is the case, the springback displacements of only the boundary nodes can be found in reduced assembly mode and the final statistical stress solution found for each part independently, thus reducing the computation power needed for the analysis.

To perform this alternate analysis, the initial analysis steps of placement and clamping in part mode are unchanged. Additional information is needed in assembly mode, however. In the fastening stage, when performing the FEM of the clamped assembly, the reduced assembly stiffness matrix $\left[\mathbf{K}_{\text {red }}\right]_{a s m}$ is used instead of the complete assembly stiffness matrix $[\mathbf{K}]_{a s m}$. The statistical clamping force calculation is unchanged.

Additionally, a new stiffness matrix of each component $j$ in its clamped position, $[\mathbf{K}]_{j, c l}$ is also to be determined. This is due to the piecewise-linear assumptions upon which the 
PLEA method relies. To simulate an inherently nonlinear assembly process, two linear processes of clamping and springback are combined by superposition. As explained in Section 4.3.3, the use of the deflected geometry for stiffness in the springback analysis approximates the nonlinearity of the fixtured assembly process.

When performing the statistical FEA solution for the springback analysis, the means and covariances of the springback forces are applied to the reduced assembly stiffness matrix to obtain the means and covariances of the springback displacements, according to Equations 5-21 and 5-22.

$$
\begin{gathered}
\left\{\mu_{\delta}\right\}_{a s m, f}=\left[K_{r e d}\right]_{a s m}^{-1}\left\{\mu_{F}\right\}_{a s m, s} \\
{\left[\Sigma_{\delta}\right]_{a s m, f}=\left[K_{r e d}\right]_{a s m}^{-1}\left[\Sigma_{F}\right]_{a s m, s}\left[K_{r e d}\right]_{a s m}^{-1}{ }^{T}}
\end{gathered}
$$

When the reduced stiffness matrix is used, the means and covariances of the springback displacements describe the springback of the boundary nodes only. To obtain the displacements throughout each part, a separate FEA solution is required for each part. The boundary displacements from the assembly solution are applied as displacement constraints to each component $j$ in its clamped position. The part stiffness matrix $[\mathbf{K}]_{j, \text { clamp }}$ is used to obtain the mean $\left\{\mu_{\delta-s p r}\right\}_{j, c}$ and covariant $\left.\mid \Sigma_{\delta-s p r}\right\rfloor_{s, c}$ springback displacement solution for each part $j$ independently. The final statistical positions of each part are found by applying the springback displacement solutions to the clamped part geometry. The statistical stresses due to springback can be found from the solution of clamped $\left\{\mu_{\delta}\right\}_{j, c}$ and $\left[\Sigma_{\delta}\right]_{s, c}$ as they are applied to the global constitutive and kinematic matrices of the clamped parts, according to Equations 5-23 and 5-24. 


$$
\begin{array}{cc}
\left\{\mu_{\sigma}\right\}_{j, c l}=[D]_{j, c l}[B]_{j, c l}\left\{\mu_{\delta}\right\}_{j, c l} & \text { Eq. 5-27 } \\
{\left[\Sigma_{\sigma}\right]_{j, c l}=[D]_{j, c l}[B]_{j, c l}\left[\Sigma_{\delta}\right]_{j, c l}[B]_{j, c l}^{T}[D]_{j, c l}^{T}} & \text { Eq. 5-28 }
\end{array}
$$

Once these stresses are known, they can be superimposed with the stress solution found in the clamping analysis to obtain the complete stress solution. A diagram of this

alternate process is given below. The principle changes occur in the inputs and outputs to Steps 7, 8, 9, and 10 . 


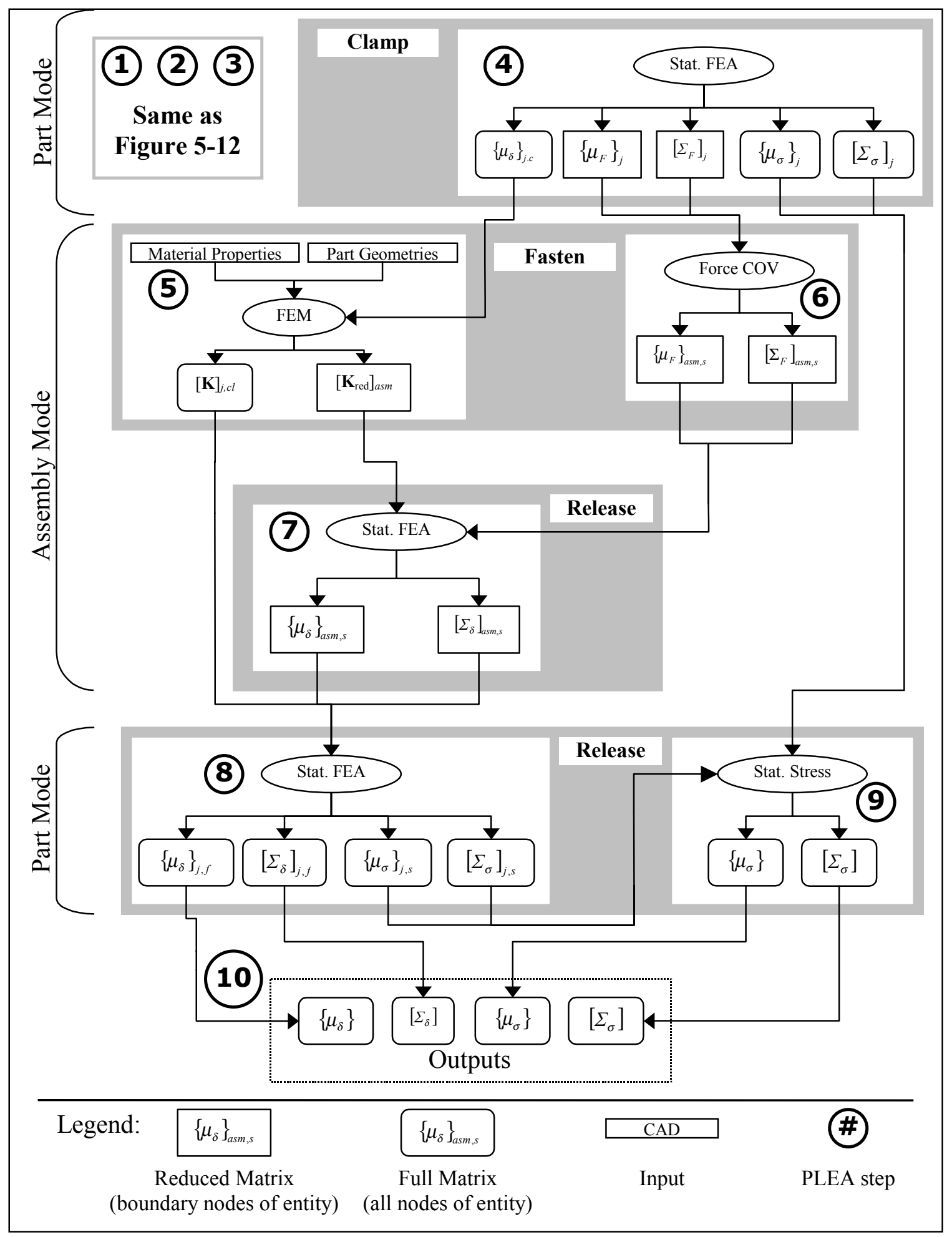

Figure 5-13: Alternate PLEA process flow diagram 


\subsection{Conclusion}

In this chapter, the PLEA steps needed to statistically model the PCFR assembly of compliant parts have been outlined. The complete PLEA process has been diagramed in process maps which can be used by the analyst or software developer for future implementation. Additionally, a separate PLEA technique has been introduced to analyze the assembly when complexity or computation requirements are limitations. It is hoped with the introduction and mapping of PLEA as developed in this chapter, many varieties of complex, compliant assemblies may now be statistically analyzed. 


\section{Chapter 6: Verification of Assembly Analysis}

In Chapter 4, Piecewise-Linear Elastic Assembly was introduced using simple beam theory. In Chapter 5, the complete PLEA process was defined and mapped. In this chapter, PLEA was verified experimentally on a real assembly. Only the mean solution will be verified in this chapter. This is done by assuming a single assembly represents the "mean" shape of a population of assemblies and comparing the measured postassembly results with those predicted by PLEA. PLEA can determine the covariance of a population of assemblies, but such a statistical determination is beyond the scope of this thesis.

The verification was accomplished through the comparisons of results obtained through analysis using ANSYS $^{\circledR}$ with results obtained through experimentation and characterization on a Brown \& Sharpe ${ }^{\circledR}$ CMM. The experimental assembly is described and the comparative results are given.

\subsection{Sample Assembly Description}

To verify the piecewise-linear analysis with real data, an assembly test fixture was fabricated to physically simulate the fixtured assembly of two sheet metal components. 


\subsubsection{Fixture Description}

The fixture consists of a flat steel base with steel risers on either side. The risers are fastened to the base by six threaded fasteners.

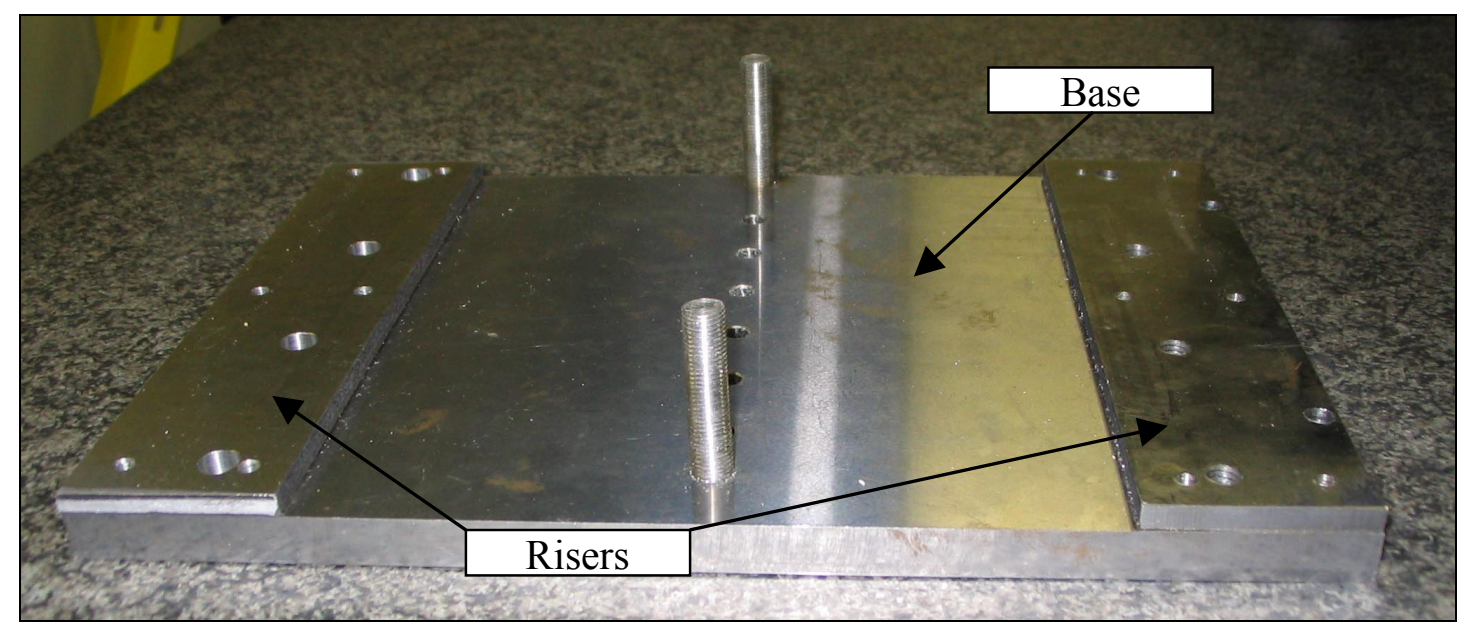

Figure 6-1: Sample assembly fixture base

To avoid assembling the parts while contacting the base and risking springback occurring towards the base, a metal standoff beam is placed on the base where assembly will occur. It is secured from motion across the base by two set screws, but can be removed by the removal of the set screws once assembly takes place. 


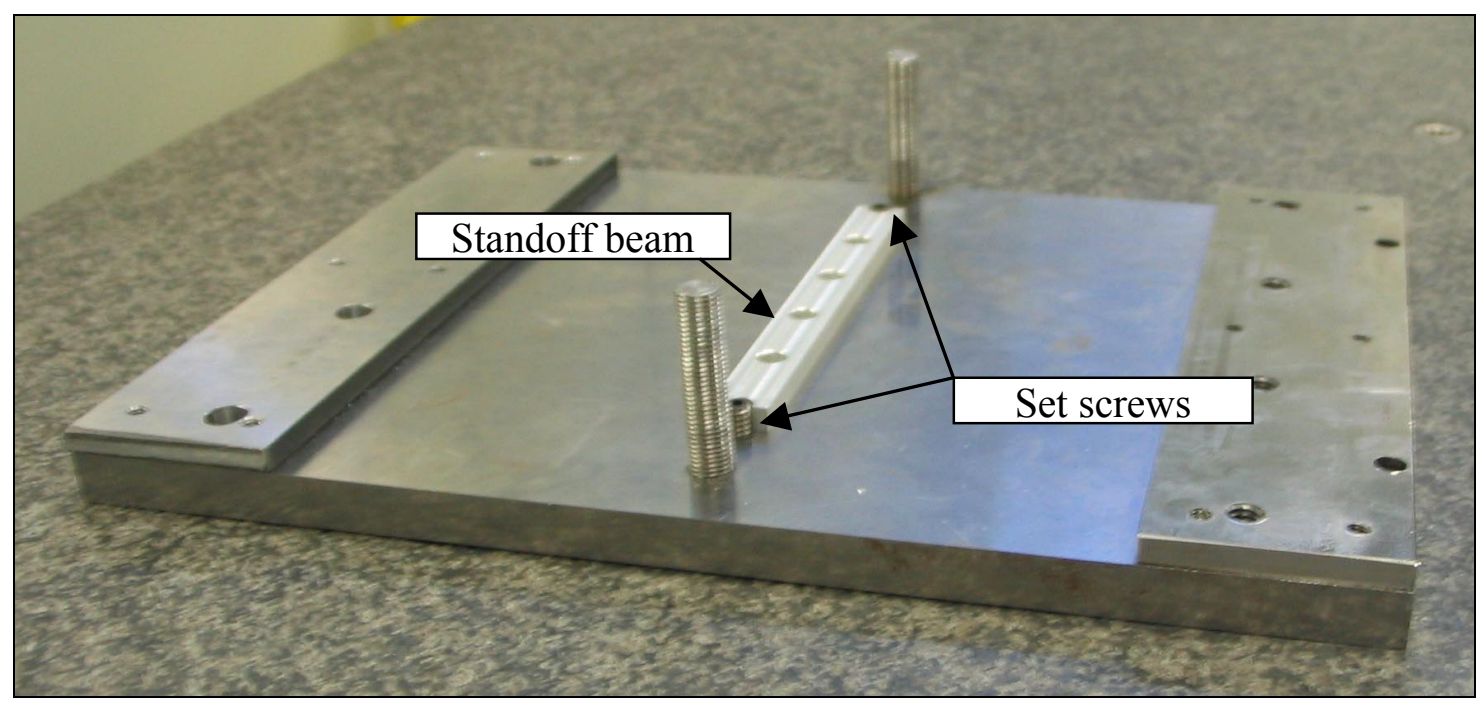

Figure 6-2: Assembly standoff beam

Atop the risers are riser clamps, which can be rigidly connected to the base by threaded fasteners. The riser clamps hold the sheet metal parts rigidly in place during and after the assembly process, simulating the placement stage of the assembly process. To constrain the sheet metal plates to the top surface of the standoff beam, a "c" channel is used. Two all-threads are welded to the middle of the steel base, which guide the "c" channel onto the standoff beam. The "c" channel is free to move up or down the all-threads freely, or it may be forced by a nut and tapered washer set to contact the standoff beam, simulating the clamping stage of the assembly process. The "c" channel web, the standoff beam and the base have coaxial thru-holes to define the rivet positions and provide relief for the drilling and riveting operations. The holes in the "c" channel web are sized for a drill bushing to ensure correct and repeatable drilling and riveting locations. 


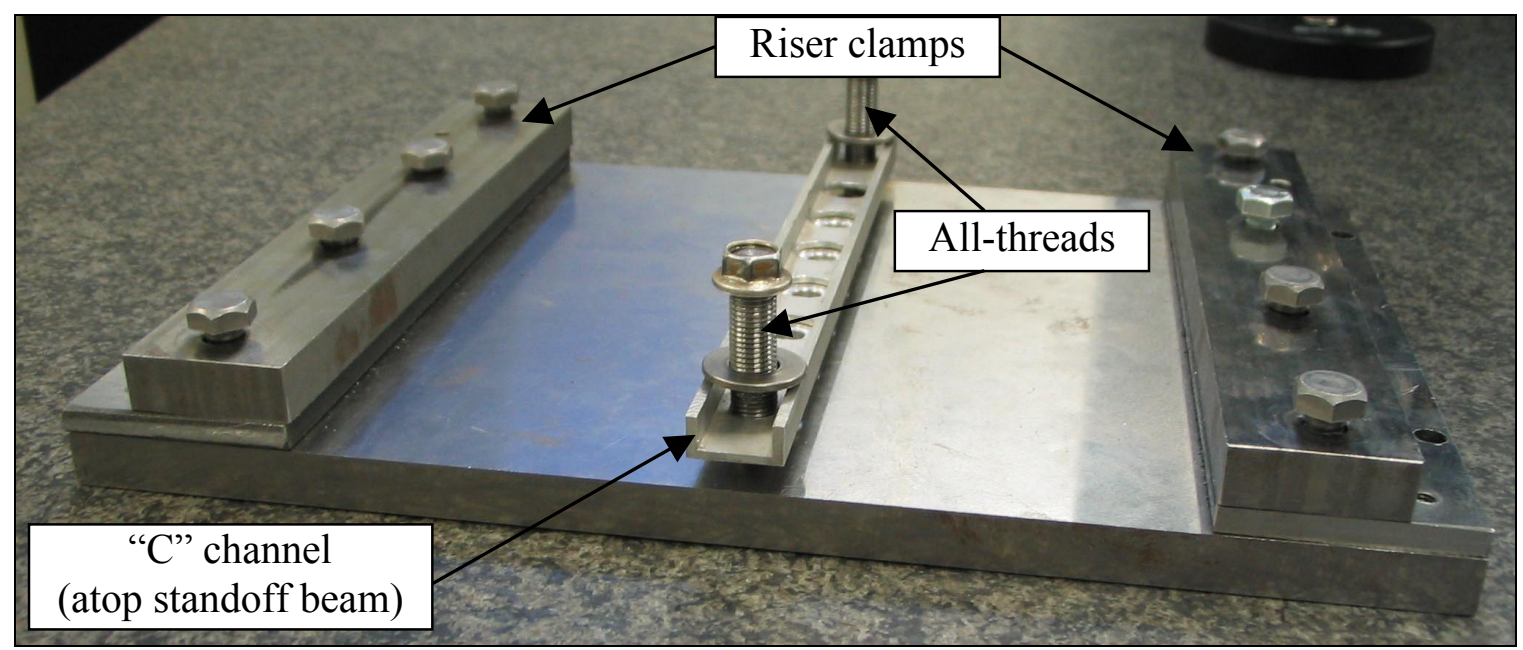

Figure 6-3: Assembly placement and clamping features

The sheet metal used in the assembly was 26 -gage ( 0.0318 inch thick) mild steel. The two assembly components were cut from the same plate and had the same orientation in the fixture to reduce variability. Each plate was 5 " wide by about 3.75 " long, and was riveted four places, 1 " apart in a line. $1 / 8$ " aluminum pop rivets were used for their light weight. A hand drill was used to drill the holes and a hand riveter was used to install the rivets.

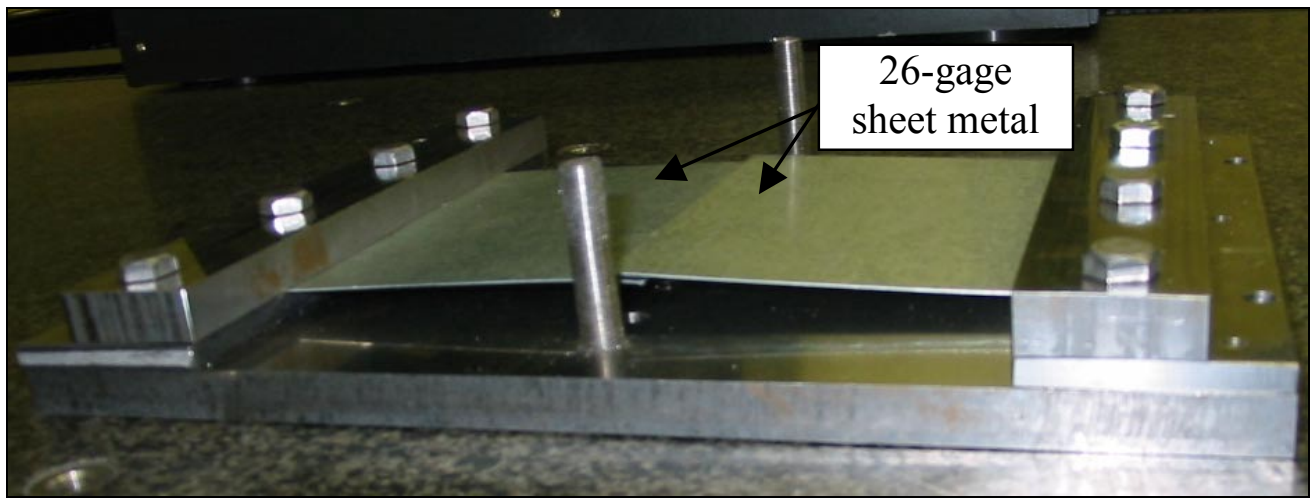

Figure 6-4: Sheet metal components in placement stage 


\subsubsection{Assembly Process}

The assembly of each component on the test fixture and characterization of all the surface variations, before and after assembly are described next.

\subsubsection{Component Placement}

The constraining of each piece of sheet metal from rigid-body motion constitutes the placement stage of the assembly process. This was done by placing each component on a riser, positioning it against the threaded fasteners of the riser clamp, and tightening the clamps to grip the part securely. To insure CMM characterization of the entire part, the component which sat lowest in the fixture was placed and scanned before the higher, overlapping component was placed and scanned.

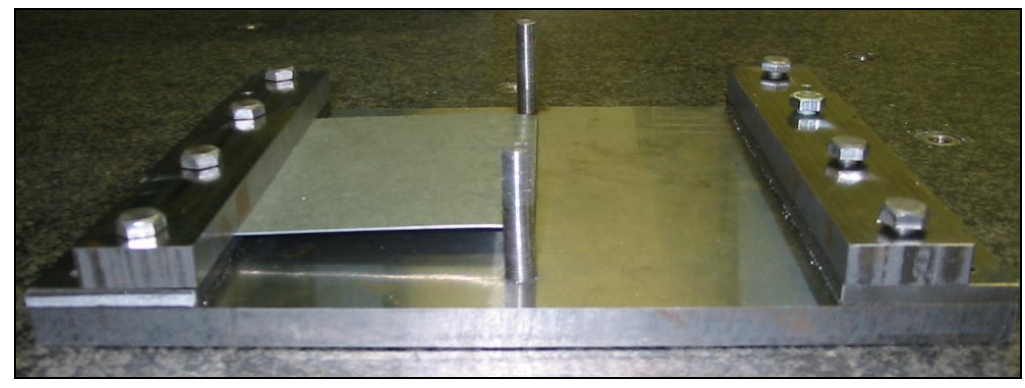

Figure 6-5: Placement of first component 


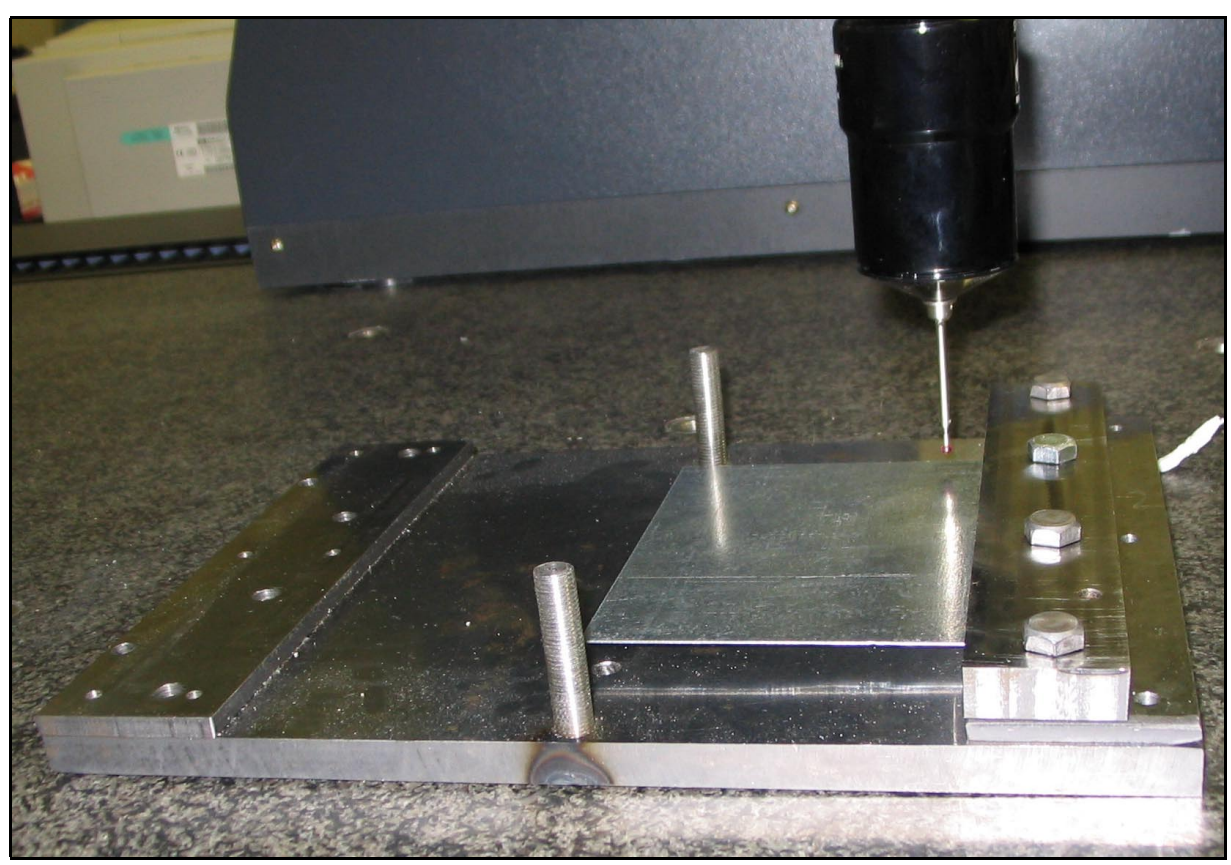

Figure 6-6: CMM scan of first component

Before any assembly takes place, the plastic behavior of the components was neutralized. The component which sat lowest was first placed into position and then forced to its clamped position on the standoff beam. After this clamping simulation, the component was released from the "c" channel and the standoff beam, but not the riser clamp. This component was then measured using the CMM. After surface measurement, the second component was placed into position and then forced to its clamped position on the standoff beam and the lower component. After this clamping simulation, both components were released from the "c" channel and the standoff beam, but not the riser clamp and the second component was measured using the CMM. These released components became the baseline for the analysis since any plastic behavior due to clamping was already neutralized. An example pre-clamping, initial position of the components is shown in Figure 6-7. 


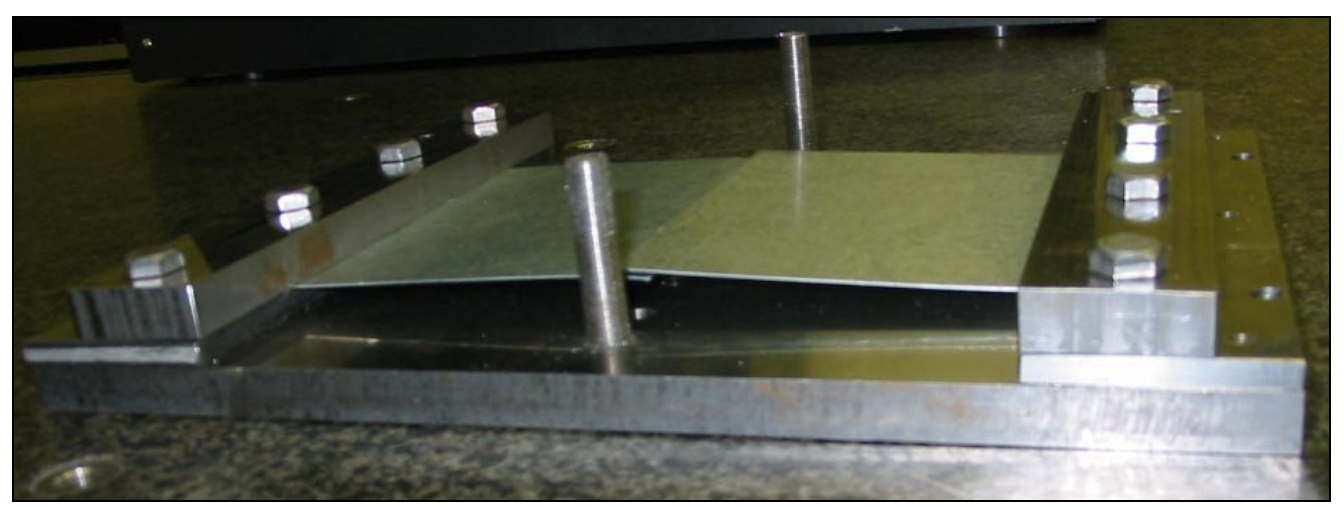

Figure 6-7: Components in their placement stage

\subsubsection{Component Clamping}

To simulate the clamping stage of the assembly process, the standoff beam was reinstalled and secured on the base by the set screws. The "c" channel was then forced against the standoff beam by the threaded nut. Because the standoff and "c" channel are flat, both forces and moments were exerted on each component to bring them into full contact. Both components were re-scanned on the CMM in their clamped positions to define the assembly deflections and rotations. An example of a clamped assembly is shown in Figure 6-8.

Because of the "c" channel clamp holds the two plates in contact with the standoff beam with downward contact force, the CMM could not access the fastener centerline. It was necessary to scan each plate and interpolate between scan points to obtain the profile of the clamped assembly. 


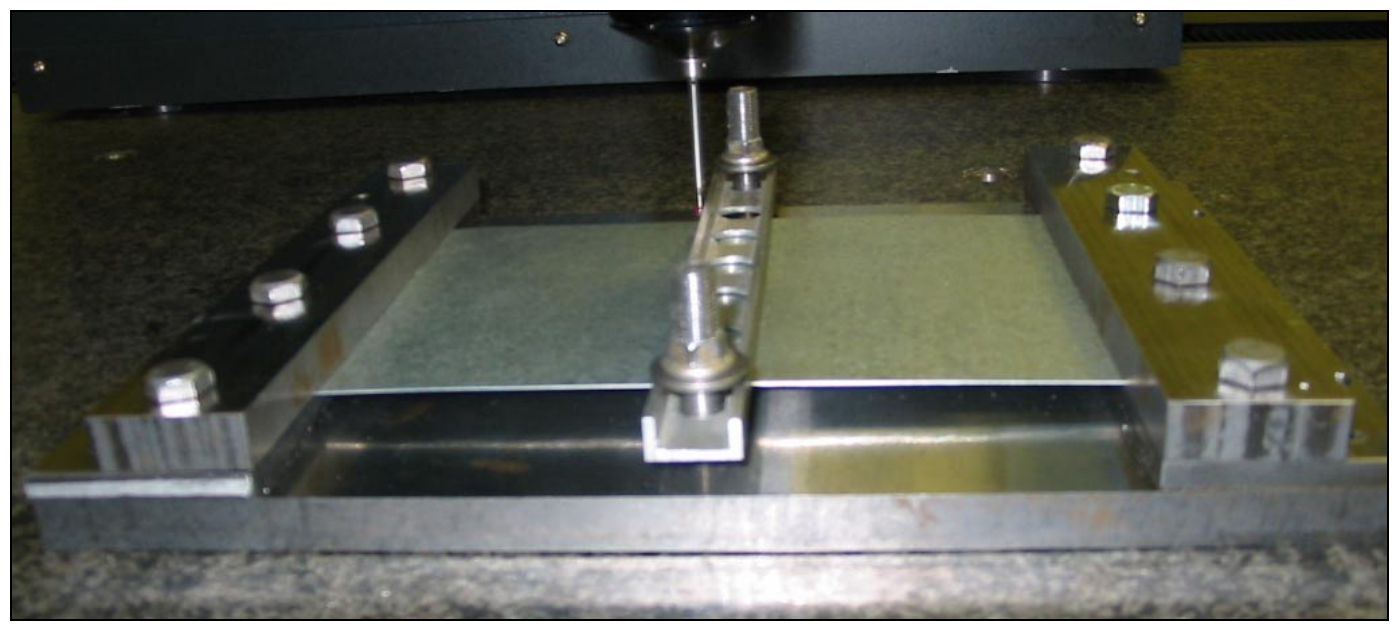

Figure 6-8: CMM scan of the clamped assembly

\subsubsection{Component Fastening}

Once forced into contact with the standoff beam by the "c" channel, the components were drilled and riveted. Using the holes drilled in the web of the "c" channel as a guide, a drill bushing was inserted and holes were drilled through both components. The drilling and riveting sequence is similar to the assembly process witnessed at Boeing, as detailed in Appendix A. One exterior hole was drilled, cleaned and riveted. The opposing exterior hole was then drilled, cleaned and riveted. Then the two interior holes were drilled, cleaned and riveted in turn. The holes were cleaned by blowing and coarse burr removal on one side. Because the drilled hole cannot be accessed easily from the base, the holes could not be completely cleaned and deburred on both sides. However, with such a tight fit between the rivet head and the hole, the effect of an undeburred side was negligible. This completed the fastening stage, and the components constituted an assembly. 

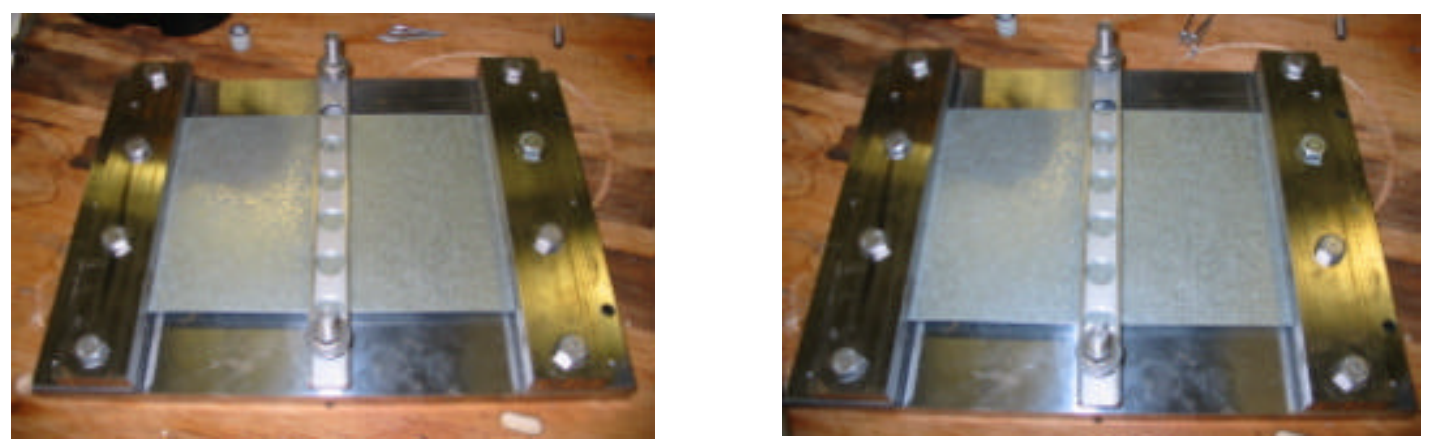

Figure 6-9: Drilling and riveting of the first location
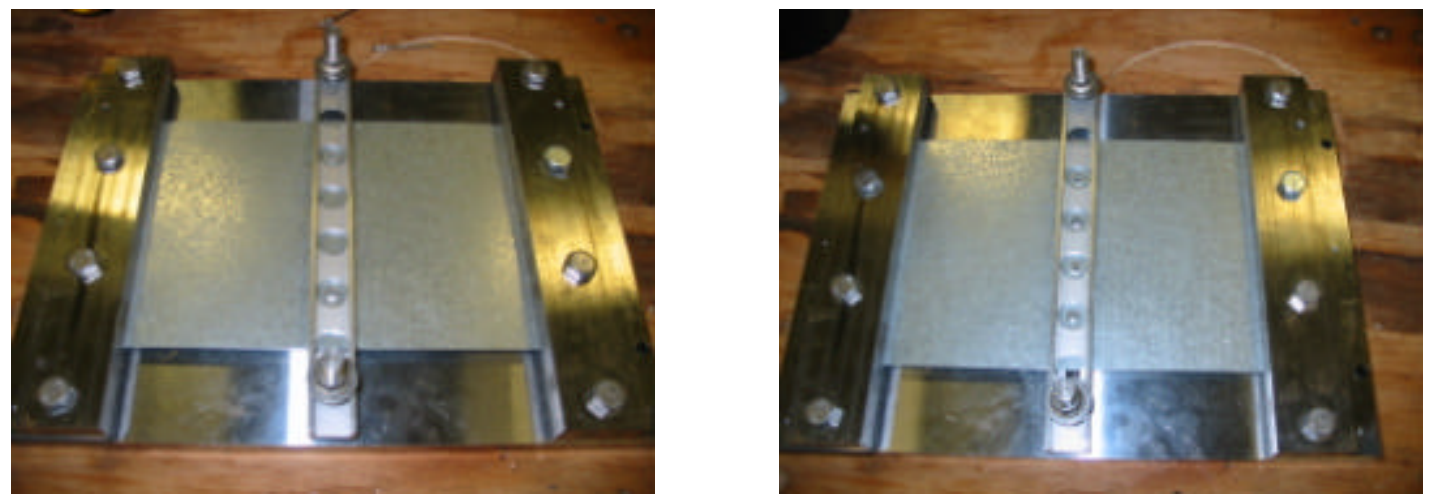

Figure 6-10: Riveting of exterior locations and completed fastening of assembly

\subsubsection{Assembly Release}

After the components were assembled, the threaded nuts constraining the "c" channel were loosened and the "c" channel and standoff beam were removed. This action constituted the release stage and the assembly experienced springback to minimize stresses. Because both risers were above the base and at different heights, the assembly experienced both force and moment springback. The whole assembly was then rescanned on the CMM. 


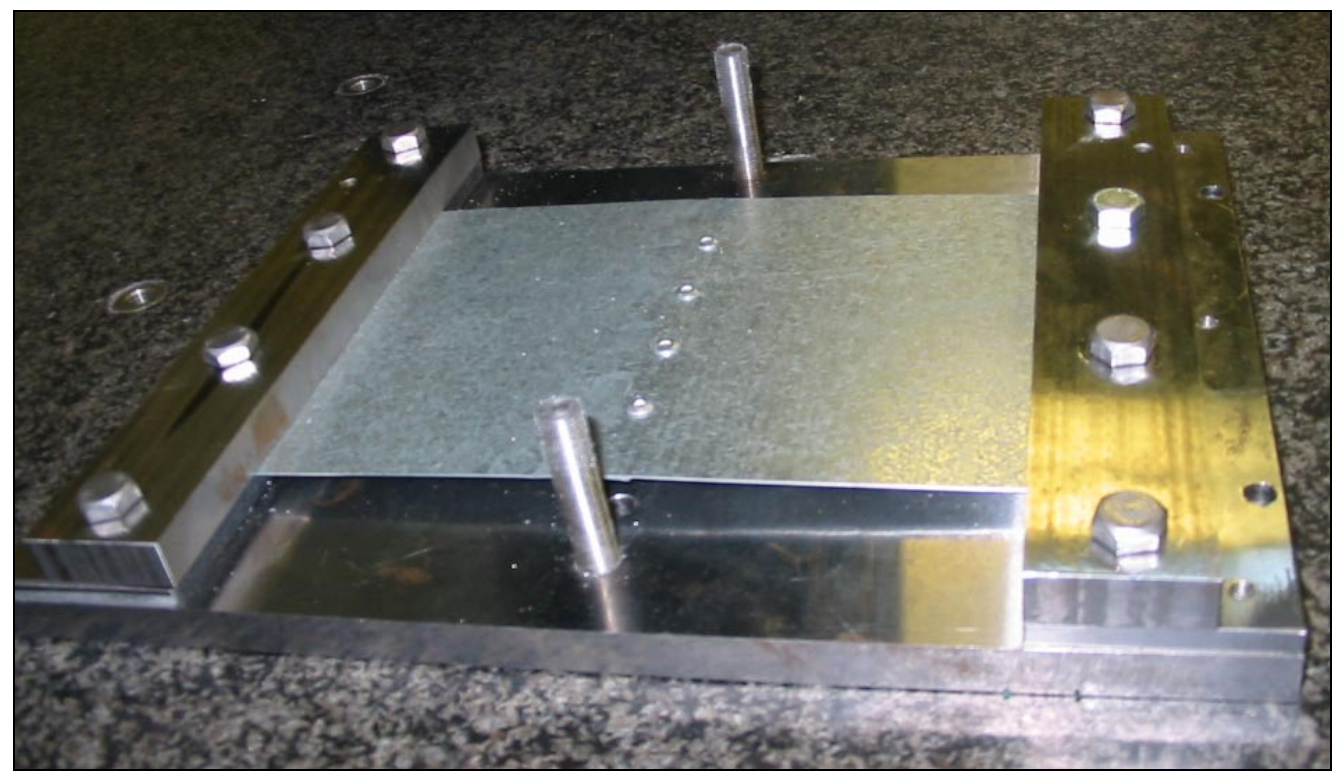

Figure 6-11: Release from the clamp and subsequent springback

\subsection{Assembly Measurement}

The analysis of this assembly process depends upon proper and accurate measurement of all components and all fixture surfaces of importance. To do this, a Brown and Sharpe ${ }^{\circledR}$ CMM was used to accurately characterize all necessary parts. The CMM measurements were used both to establish boundary conditions for the analysis as well as for checking deflection and springback results. Controlled by PC-DMIS ${ }^{\circledR}$ version 3.0 software, the CMM scanned each component at 12 scan points per 1" run. The CMM was calibrated before each series of scans. It is claimed to have a resolution of 0.0004 ", but for this experimentation an accuracy of 0.001 " is sufficient, so the CMM results were rounded for the inputs to the analysis. 


\subsubsection{Fixture Scans}

The top of the risers and the top of the standoff were the first surfaces scanned with the CMM. This characterization showed the heights of the boundary conditions in the placement and clamping stage. By using these heights, the sections of the components that couldn't be measured when they were in the clamps, were inferred. It was necessary to characterize these surfaces to verify the fixture surfaces have some measurable error.

\subsubsection{Component Scanning and Modeling}

To accurately assign boundary conditions for the analysis, surface scans were obtained from the PCFR assembly process corresponding to the components in their located positions, the assembly in its clamped position and the assembly in its released, postspringback position. The springback surface scans were for comparison to the analytical predictions. In the CMM scans, the scan points did not always coincide with an orthogonal coordinate system native to the fixture. This is because the CMM scanned the area within the user-defined scan boundary without any specified scan path constraints (with the exception of the 12 scan "hits" per inch constraint). Uniform spacing of scan points eases FEA modeling, so the scan points were cast into points on an orthogonal coordinate system native to features on the fixture. Through 2-D linear interpolation and rounding, the scan points obtained from the CMM were successfully mapped onto this new coordinate system. With a node somewhere inside a 0.083 " area, linear interpolation was assumed to provide sufficient approximation for actual nodal position. 


\subsubsection{Clamping Analysis}

After coordinate transformation, both rectangular plates were modeled in ANSYS ${ }^{\circledR}$ with four-node quadrilateral shell elements of uniform $1 / 4$ inch by $1 / 4$ inch nominal edge length. ANSYS $^{\circledR}$ shell elements (Shell 63) have the ability to model in-plane loads and in-plane shear in thin members. They are well suited for analysis of compliant sheet metal parts, shown in Mortensen [2002]. After transformation, each component had 280 elements and 315 nodes. Sample initial positions of the components as measured by CMM and modeled in ANSYS ${ }^{\circledR}$ are shown in Figure 6-12 and Figure 6-13.

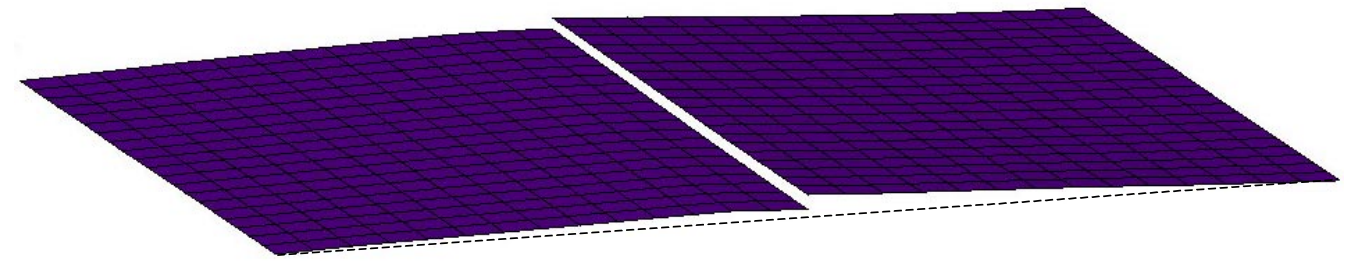

Figure 6-12: Sample components as modeled in ANSYS $^{\circledR}$

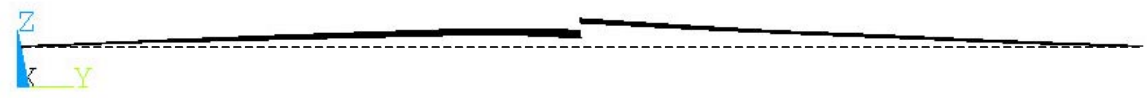

Figure 6-13: Sample component gap as seen in $\operatorname{ANSYS}^{\circledR}$

The scan points obtained from the standoff beam of the fixture were used to determine the final positions of the 21 assembly nodes of each component. When these positions and the initial, placed positions of the components were known, the $z$-values were subtracted to find the prescribed $z$-displacements to simulate the clamping stage in ANSYS $^{\circledR}$. Additionally, when the actual assembly components were in their clamped 
position, a scan was obtained for the purpose of error checking, since it was assumed the analysis shape should coincide with the actual clamping shape.

One of the significant findings of this analysis is the prescribed conditions must be a simulation of actual loading characteristics. In our case, both edges exhibit slight motion in the $y$-direction as they are clamped. By applying only a $z$-displacement and a rotation about the $x$-axis to the closure nodes, the conditions of clamping and "exact-constraint" of the nodal motion are properly described. This differs from an "over-constrained" displacement applied to the closure nodes, where displacement constraints would be applied to all six degrees of freedom (translation in $x$-, $y$ - and $z$-directions and rotation about $x$-, $y$ - and $z$-axes). An example of this, pertinent to our analysis is illustrated below. One cross section of one plate yields a profile similar to that given in Figure 6-14 and Figure 6-15. Due to the nature of the riveting process, both plates must overlap the fixture. To perform an exactly-constrained closure step consistent with our clamping process, a negative displacement constraint in the $z$-direction and a positive rotation about the $x$-axis are applied to the closure node.

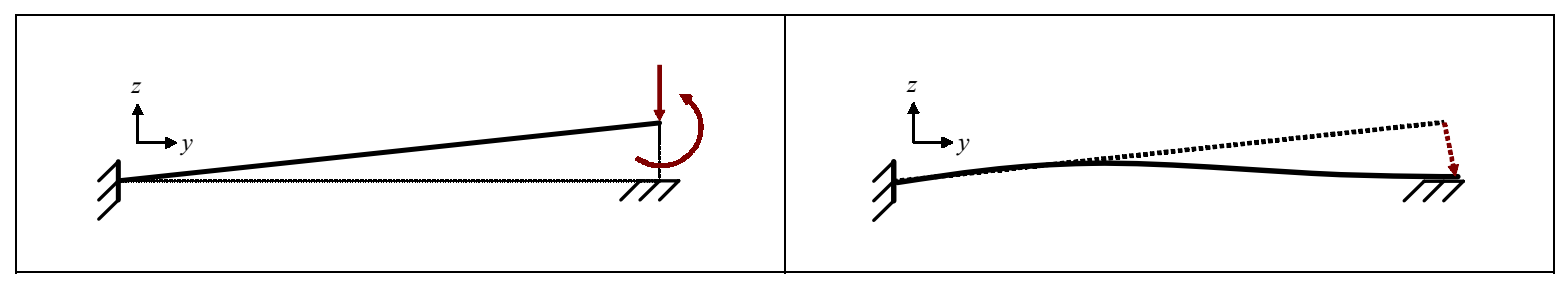

Figure 6-14: Exact constraint on a closure node 


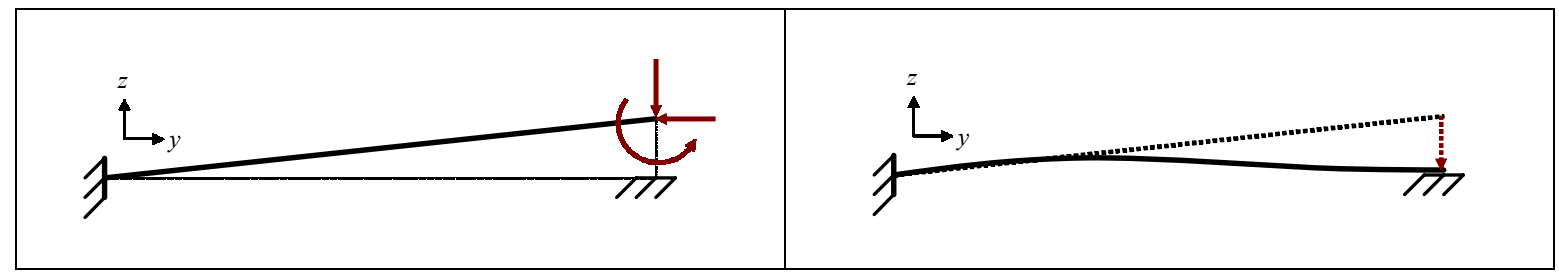

Figure 6-15: Over constraint on a closure node

Because the part is free of constraints in the $y$-direction, the closure node is expected to translate. This nodal translation is not significant, and has negligible impact on further analysis steps. However, any constraint in the $y$-direction, even a definition of zero displacement, is very significant. Because the part is stiff in the $y$-direction, any displacement constraints can cause large assembly forces which, due to the initial geometry, may not be limited to the $y$-direction. Even when the expected displacement in the $y$-direction is known, it is discouraged from specifying it as a displacement constraint, since round-off error may occur in FEA programs, leading to unintentional forces.

To demonstrate the effects of exact- and over-constraint, both loading techniques will be briefly compared. A sample clamping analysis was performed on one plate and both over-constrained and exact-constrained displacement loads were applied. The graphs in Figure 6-16 illustrate the difference between the actual scans obtained from the clamping stage and those simulated with both loading conditions. Note that the clamped positions of both loading conditions are identical on the ends where the loads are applied, but have great differences in the middle. From the images, it is certain that the exactly-constrained closure is a better prediction of the actual shape than the over-constrained closure and any over-constrained loads must be avoided. 


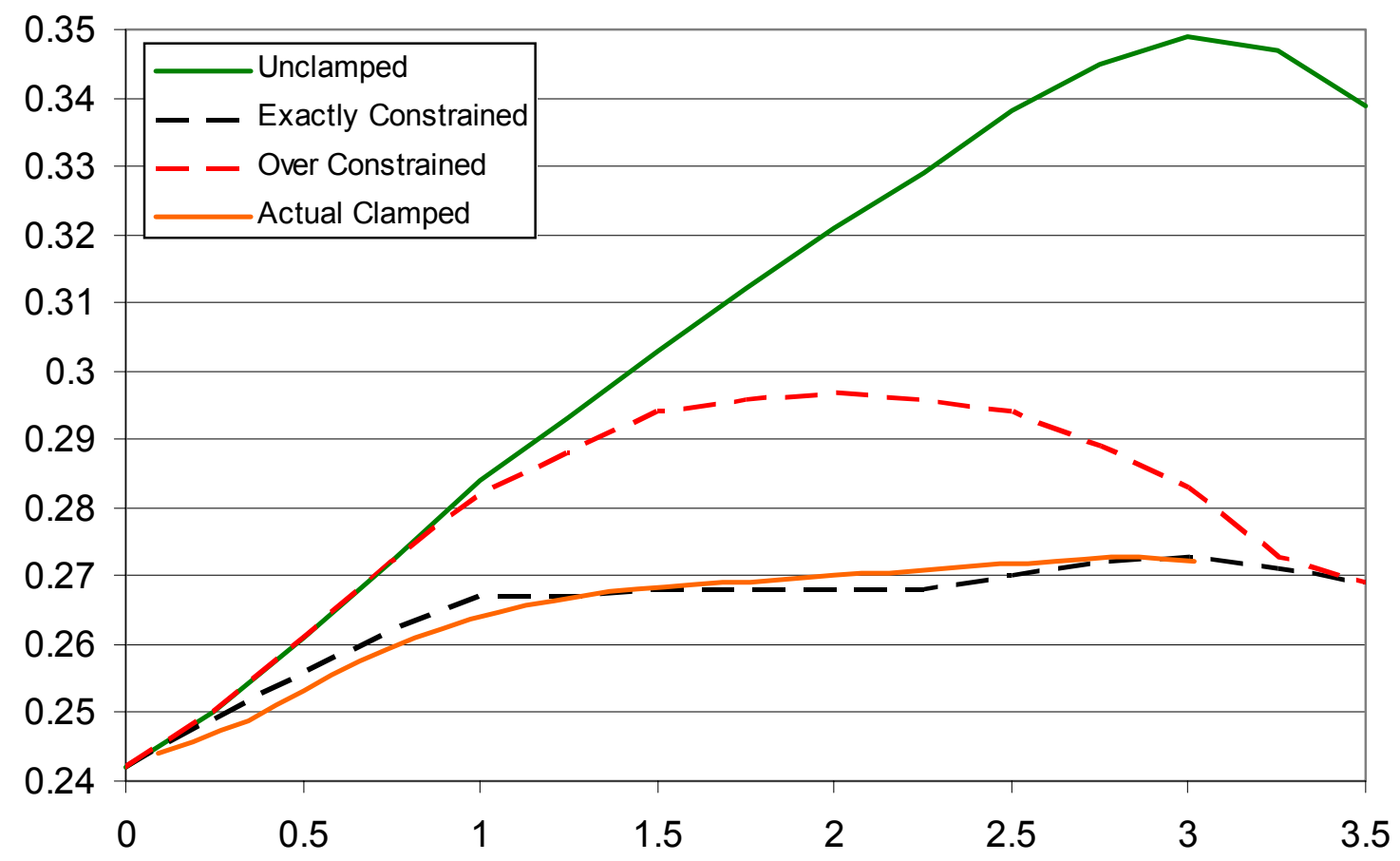

Figure 6-16: Sample cross-section of one plate comparing clamping analysis methods

The results pertinent to this analysis step are the final shapes of both components as well as the assembly forces required to deform the components to their specified positions. If an overall stress solution is also required, the nodal stresses would be determined for both components and the quantities saved for post-processing.

\subsubsection{Release and Springback Analysis}

Once the components were analyzed in their clamped positions, the assembly was analyzed for springback. ANSYS ${ }^{\circledR}$ has the ability to output the nodal deflections as a text file. When the initial nodal positions are added to the corresponding nodal deflections, a deflected part is obtained, which can be input back into ANSYS ${ }^{\circledR}$ for fastening and springback analysis. The two components were input into $\mathrm{ANSYS}^{\circledR}$ as two stress-free plates in their deflected positions. The assembly nodes were coupled in all DOF by the 
ANSYS ${ }^{\circledR} \mathrm{CP}$ command to simulate the fasteners joining the two plates at each closure node. Although neighboring closure nodes might not be coincident due to elongation because of our exact-constraint clamping loads, the distance between them is small enough that the effect is negligible. In all the analyses conducted in ANSYS ${ }^{\circledR}$, the maximum horizontal difference between closure nodes never exceeded 0.005 ". These two deformed components, with coupled DOFs at their corresponding closure nodes, constitute the pre-springback assembly.

In this stage, the assembly was modeled as stress-free. This is due to one of the fundamental assumptions of the PLEA method, which is, that we can simulate a nonlinear springback effect by superimposing linear clamping and release analyses. To the assembly model, fixed boundary conditions were applied to the 42 nodes ( 21 on each component) along the two supporting edges. The complements of the assembly forces, calculated in the clamping stage, were applied to the corresponding closure nodes of the assembly and the analysis performed.

\subsection{Analysis Results}

To show the effects of greater offsets on the final geometry predictions, three load cases were analyzed, each exhibiting differing offsets from ideal geometry. Each load case will be detailed and the results discussed.

These load cases were analyzed according to the methods detailed previously in this chapter. Displacement and rotation loads were applied to each endpoint to simulate the 
clamping loads. After the clamping displacement and assembly forces were calculated, the shape of the plates due to clamping were plotted and compared to the actual clamping shape. With the completion of the clamping analysis, the deformed positions of the plates were modeled and the reverse assembly loads were applied. The results from this analysis were compared to the actual post-assembly scans. It should be noted that these plots always correspond to the top surfaces of the parts, due to the nature of CMM measurement. When the parts are clamped together, the difference between the closure nodes usually corresponds to the thickness of the upper part.

To compare the predicted and actual results, different cross-sections will be analyzed. The plates are 5" wide with closure points at $x=1.0, x=2.0, x=3.0$ and $x=4.0$. To show a wide range of effects, the edge at $x=0.0$, the riveted cross-section at $x=1.0$ and the mid-plane at $x=2.5$ will be graphed and the nodal results at the gap will be tabulated. Tabular results which are estimates using extrapolation will be given in parentheses. An illustration of these cross-sections relative to the assembled parts is given in Figure 6-17.

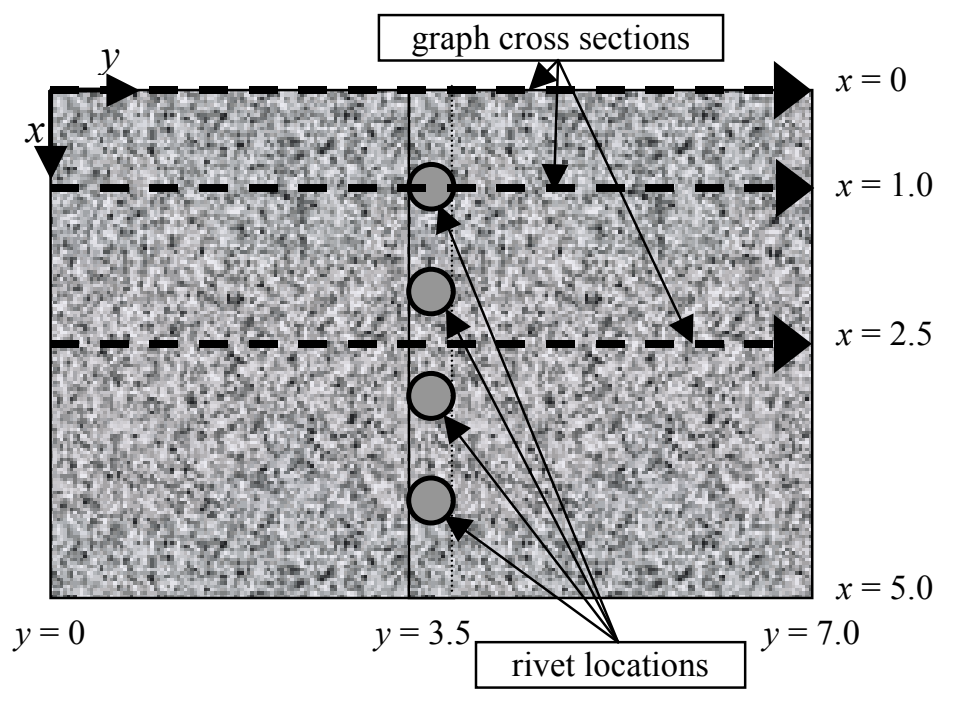

Figure 6-17: Graph section illustration 


\subsubsection{Load Case 1-Near Ideal}

The first analysis case is the nearest to the ideal pre-assembly shape. Both risers and the standoff beam are nominally the same 0.25 " offset from the base. Additionally, both components exhibit little dimensional variation from ideal, meaning they exhibit a near constant stiffness relationship along the entire range of deflection. At the maximum

offset, the deflection-to-part-length ratio, ${ }^{\delta} / \mathrm{L}$, is less than the $1 / 100$ ratio common to tolerance specifications. With the slight imperfection of the fixture and the parts, this load case is one that would be expected in a production environment. The analysis of this load case can be seen in Figure 6-19. The vertical deflections have been scaled by 200 for this graph. Note that the graphs do not touch, even in the clamped position. This is because the parts overlap and the offset due to part thicknesses are more noticeable in this load case. 


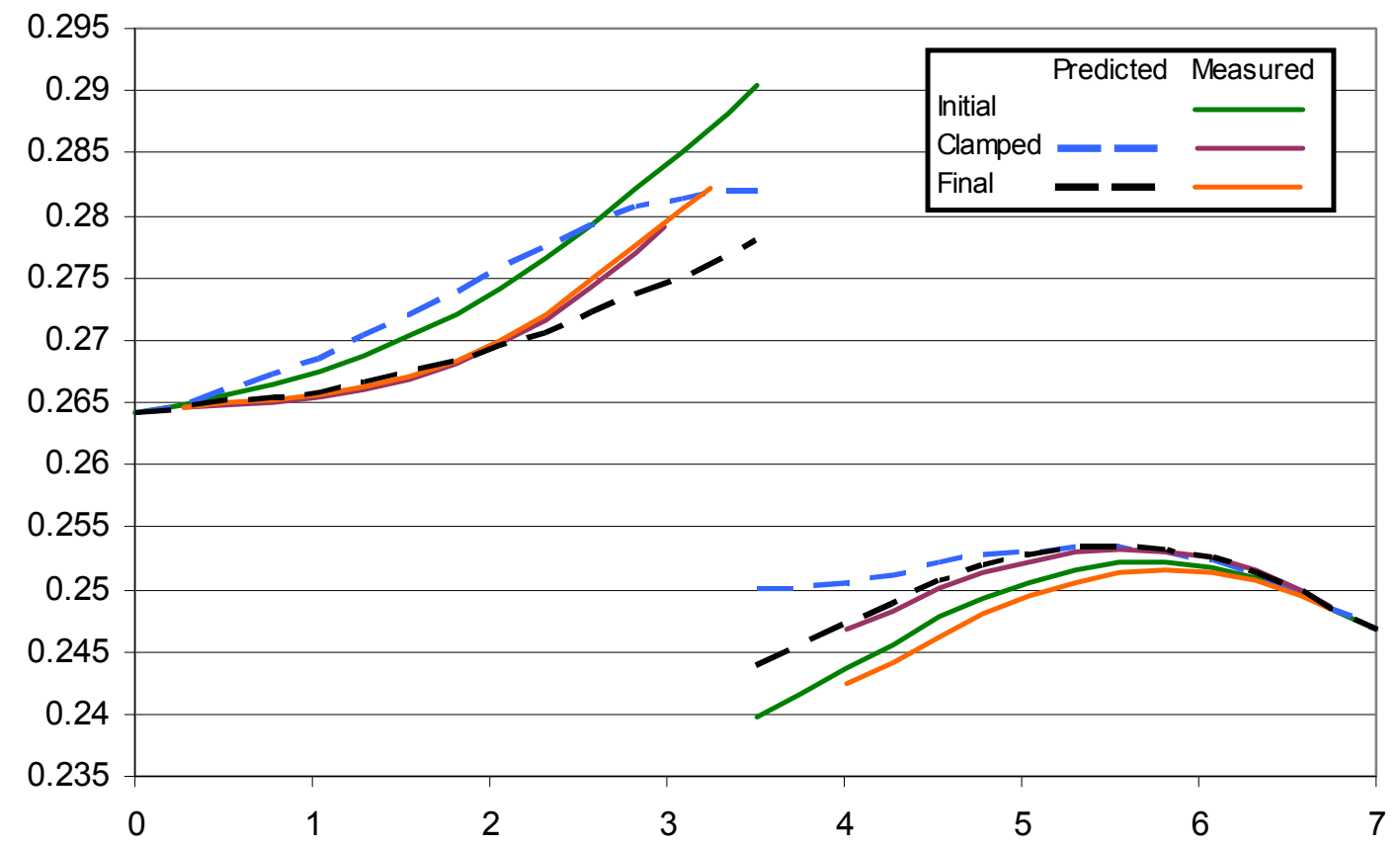

Figure 6-18: Load case 1 cross section results at $x=0.0$

Table 6.1: Load case 1 cross section tabular results at $x=0.0$

\begin{tabular}{|l|c|c|}
\hline & Left Plate & Right Plate \\
\hline Initial Position & 0.2904 & 0.2398 \\
\hline Clamped Position & 0.282 & 0.25 \\
\hline Offset Ratio $\mathbf{~} \mathbf{L}$ & 0.0024 & 0.0029 \\
\hline Final Predicted Position & 0.278 & 0.2439 \\
\hline Actual Measured Position & $(0.2851)$ & $(0.2393)$ \\
\hline Absolute Error & $\mathbf{( - 0 . 0 0 7 1 )}$ & $(0.0046)$ \\
\hline
\end{tabular}




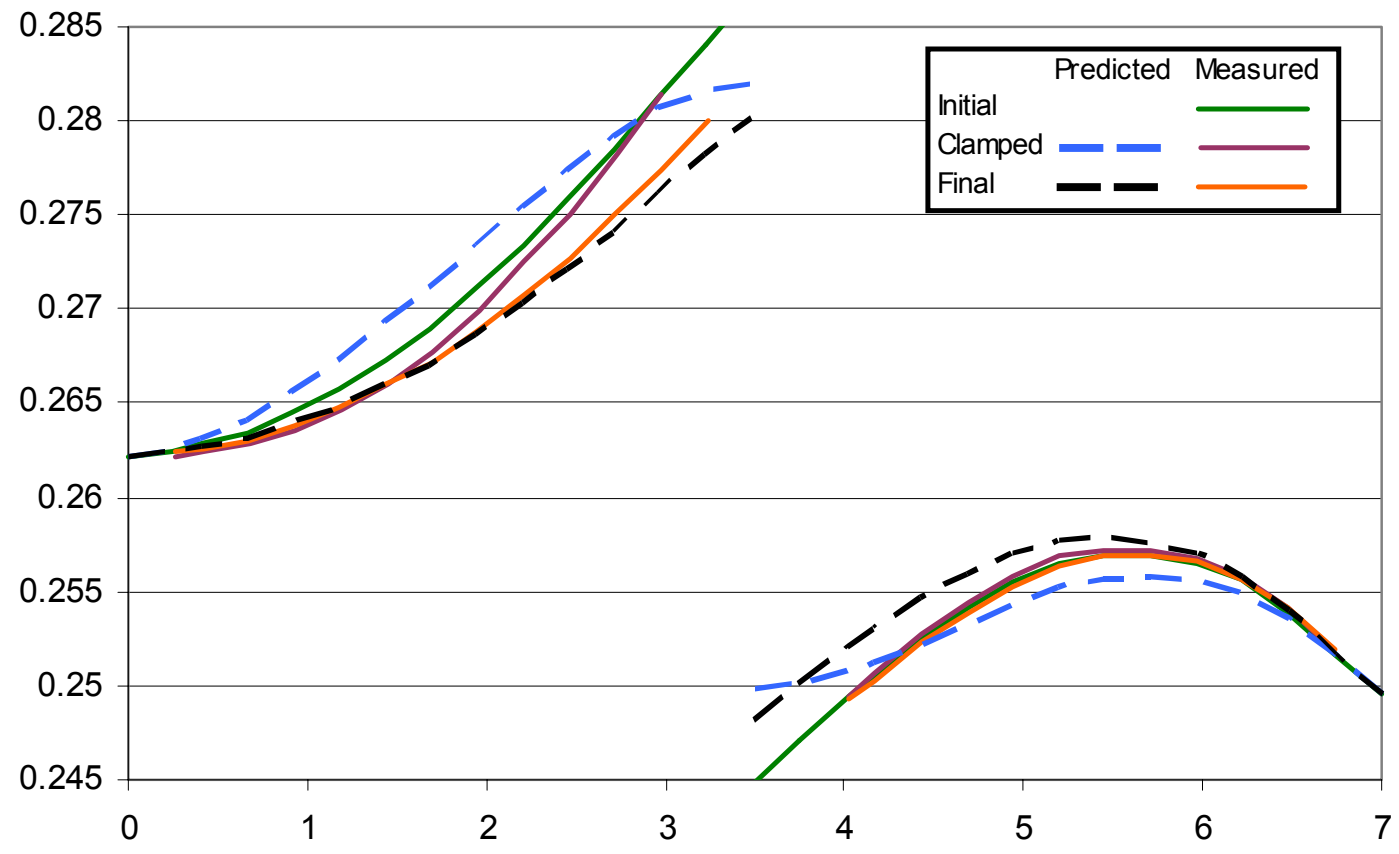

Figure 6-19: Load case 1 cross section results at $x=1.0$

Table 6.2: Load case 1 cross section tabular results at $x=1.0$

\begin{tabular}{|l|c|c|}
\hline & Left Plate & Right Plate \\
\hline Initial Position & 0.2869 & 0.2449 \\
\hline Clamped Position & 0.2819 & 0.2499 \\
\hline Offset Ratio $\mathbf{~} \mathbf{L}$ & 0.0014 & 0.0014 \\
\hline Final Predicted Position & 0.2802 & 0.2482 \\
\hline Final Measured Position & $(0.2830)$ & $(0.2453)$ \\
\hline Absolute Error & $(-0.0028)$ & $(0.0029)$ \\
\hline
\end{tabular}




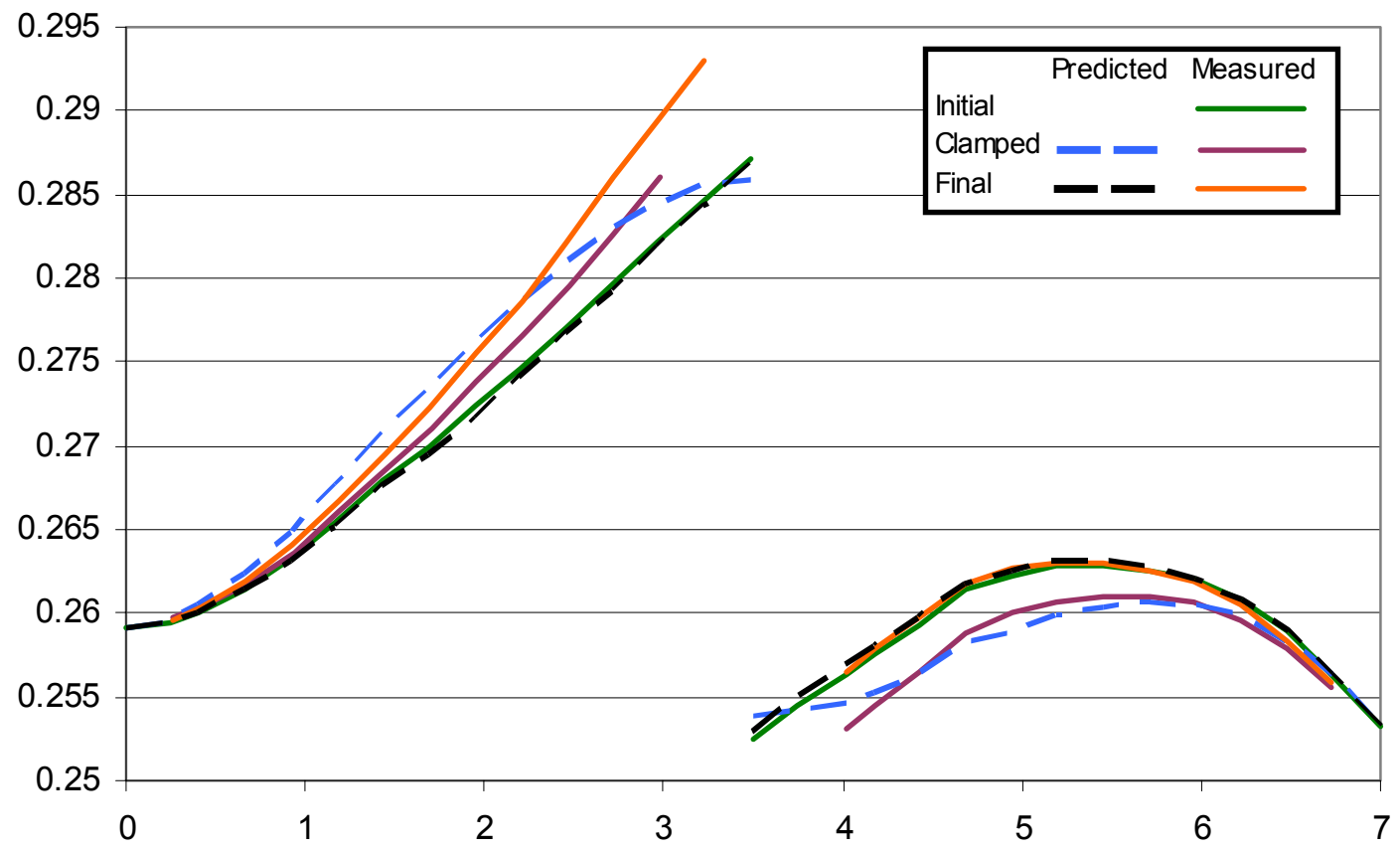

Figure 6-20: Load case 1 cross section results at $x=2.5$

Table 6.3: Load case 1 cross section tabular results at $\boldsymbol{x}=\mathbf{2 . 5}$

\begin{tabular}{|l|c|c|}
\hline & Left Plate & Right Plate \\
\hline Initial Position & 0.2871 & 0.2525 \\
\hline Clamped Position & 0.2858 & 0.2538 \\
\hline Offset Ratio $\mathbf{8}$ L & 0.0004 & 0.0004 \\
\hline Final Predicted Position & 0.2868 & 0.2530 \\
\hline Actual Measured Position & $(0.2966)$ & $(0.2529)$ \\
\hline Absolute Error & $\mathbf{( - 0 . 0 0 9 8 )}$ & $(0.0001)$ \\
\hline
\end{tabular}

This load case was the most difficult to analyze, since the deflection range is on the order of the flatness of the parts. The biggest obstacle to accuracy in this load case was determining the clamping constraints. There are two reasons for inaccuracy in assigning the translational and rotational clamping constraints. First, since the CMM probe cannot measure the plates in their clamped position nearest the clamps boundary, the clamping displacements and rotations can only be supposed through extrapolation. This is especially true of the upper component, which is constrained against the lower 
component and not the standoff beam. Second, because the parts exhibit little overall deflection, assigning the rotational constraint is difficult, since the parts are not at a $0^{\circ}$ absolute angle at the ends. In other load cases, a $0^{\circ}$ absolute angle is a good approximation to the actual angle of the clamped position. These two factors make assigning boundary conditions for this case little more than trial-and-error. A slight error in assigning the clamping conditions may lead to inaccuracies in the final predictions. Regardless of the inaccuracies in modeling this analysis, it gives good results. One insight which comes from analyzing this load case is that while the deflection to part length ratio is in the range expected in production environments, the measurement of this load case needs to be very accurate and conducted in a controlled environment. In the next chapter, where a much larger production assembly is analyzed, measurement error was not a limitation.

\subsubsection{Load Case 2-Component Warping}

In the second load case, both risers and the standoff beam are nominally the same offset from the base. However, both components exhibit greater dimensional variation from their ideal geometries. In this case, each component has $\delta / L$ ratios of $1 / 10$ to $3 / 10$. This is a load case which may be encountered in a production environment where the parts are moderately warped prior to assembly. The vertical deflections have been scaled by about 50 for these graphs. 


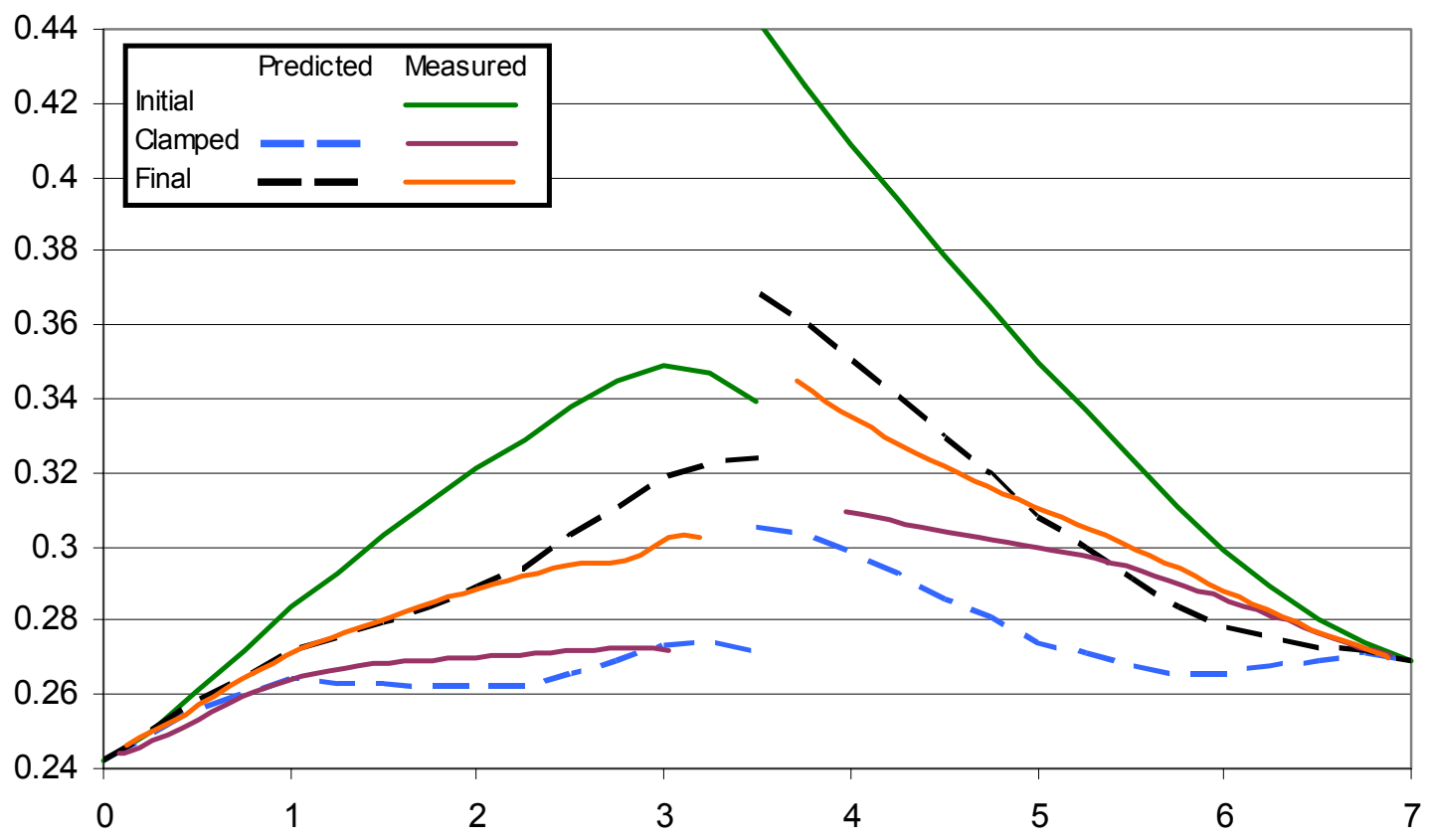

Figure 6-21: Load case 2 cross section results at $x=0.0$

Table 6.4: Load case 2 cross section at $x=0.0$ tabular results

\begin{tabular}{|l|c|c|}
\hline & Left Plate & Right Plate \\
\hline Initial Position & 0.339 & 0.442 \\
\hline Clamped Position & 0.272 & 0.305 \\
\hline Offset Ratio $\mathbf{~} \mathbf{L}$ & 0.019 & 0.039 \\
\hline Final Predicted Position & 0.324 & 0.368 \\
\hline Actual Measured Position & $(0.305)$ & $(0.349)$ \\
\hline Absolute Error & $(0.019)$ & $(0.019)$ \\
\hline
\end{tabular}




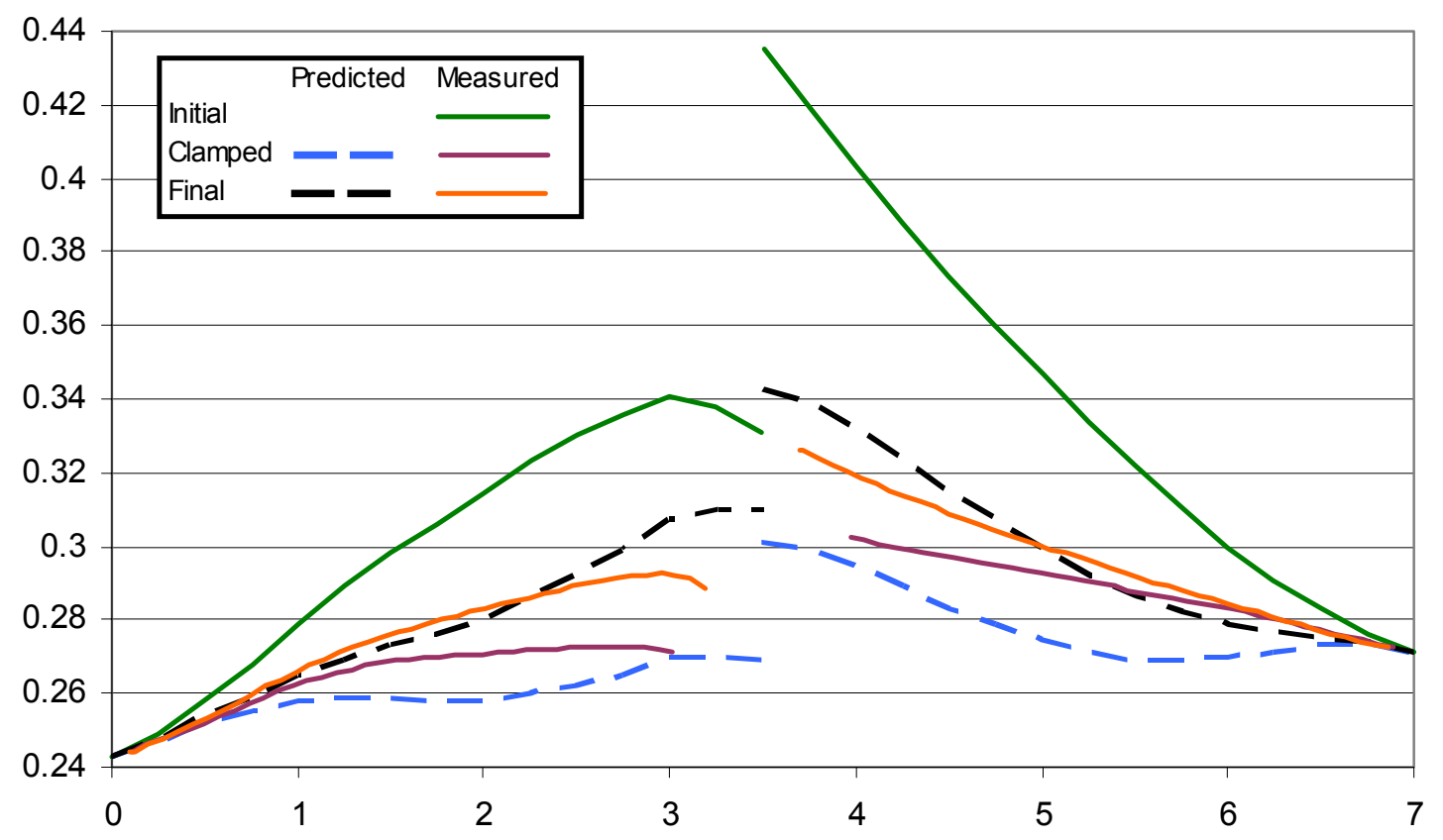

Figure 6-22: Load case 2 cross section results at $x=1.0$

Table 6.5: Load case 2 cross section at $x=1.0$ tabular results

\begin{tabular}{|l|c|c|}
\hline & Left Plate & Right Plate \\
\hline Initial Position & 0.331 & 0.435 \\
\hline Clamped Position & 0.269 & 0.301 \\
\hline Offset Ratio $\mathbf{~} \mathbf{L}$ & 0.0177 & 0.0383 \\
\hline Final Predicted Position & 0.310 & 0.342 \\
\hline Actual Measured Position & $(0.288)$ & $(0.330)$ \\
\hline Absolute Error & $(0.022)$ & $(0.012)$ \\
\hline
\end{tabular}




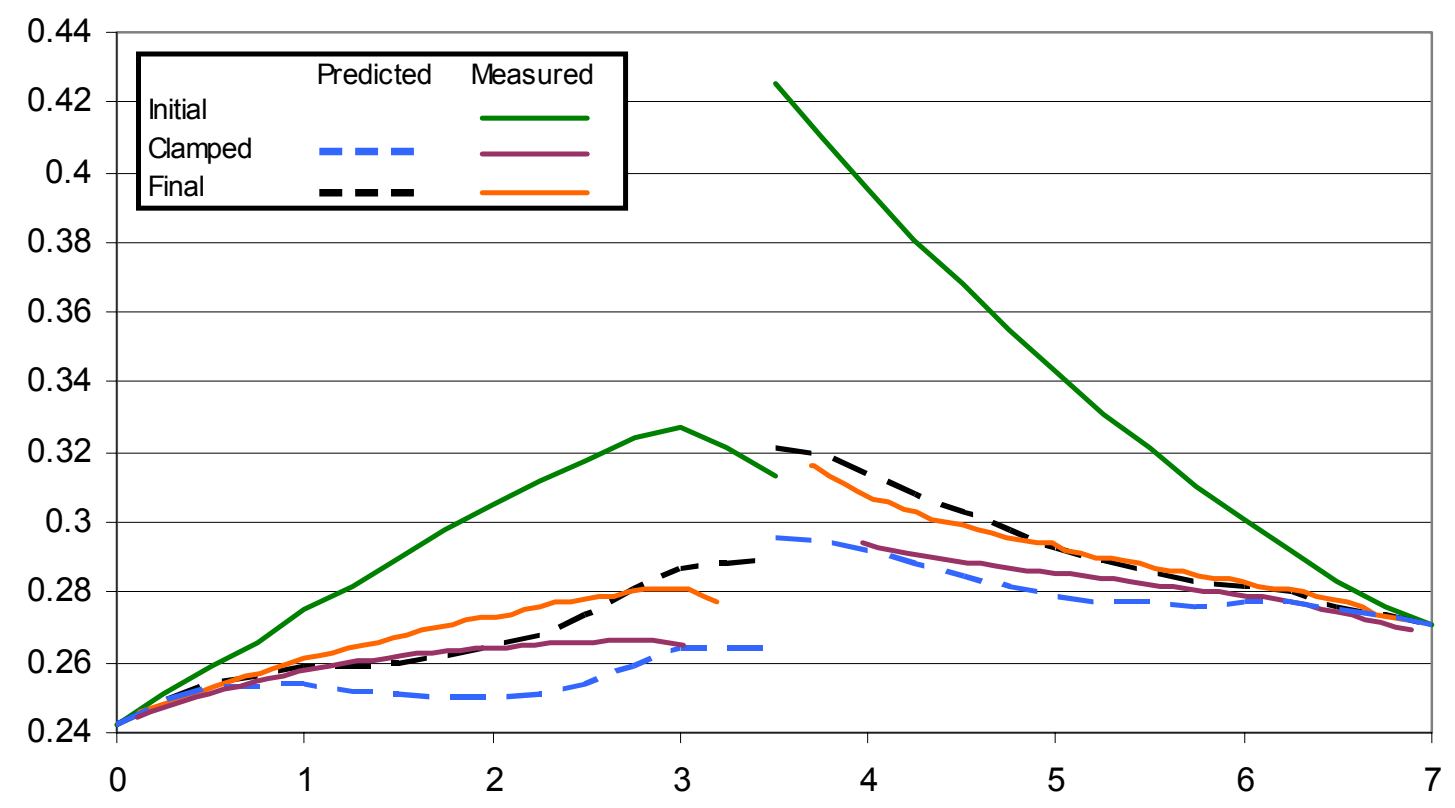

Figure 6-23: Load case 2 cross section results at $x=2.5$

Table 6.6: Load case 2 cross section at $x=2.5$ tabular results

\begin{tabular}{|l|c|c|}
\hline & Left Plate & Right Plate \\
\hline Initial Position & 0.313 & 0.425 \\
\hline Clamped Position & 0.264 & 0.296 \\
\hline Offset Ratio /L $_{\text {L }}$ & 0.0140 & 0.0369 \\
\hline Final Predicted Position & 0.289 & 0.321 \\
\hline Actual Measured Position & $(0.277)$ & $(0.323)$ \\
\hline Absolute Error & $(0.012)$ & $(-0.002)$ \\
\hline
\end{tabular}

From the above figures, the PLEA predictions are accurate in some cases, but exhibit inaccuracies in others. This may be due to the clamping inaccuracies. Although the boundary conditions are met, in that the parts are brought to a specified point with a specified rotation, the interior nodes do not always correspond to the actual measurements. This might be due to the large displacements necessary to perform the clamping analysis. Large displacements could cause a nonlinear stiffness relationship in the components, which would lead to inaccuracies. 


\subsubsection{Load Case 3- Great Initial Offset}

In the third load case, one riser is located at an approximate 0.25 " offset from the base, the other riser is located at an approximate 0.75 " offset from the base, and the standoff beam is located at an approximate 0.38 " offset from the base. Both components will need great displacement to come into contact with the standoff beam, meaning neither will maintain a linear stiffness relationship along the range of deflection. The $\frac{\delta}{\mathrm{L}}$ ratios in this load case can be as great as $1 / 8$. The vertical deflections have been scaled by about 10 for these graphs. 


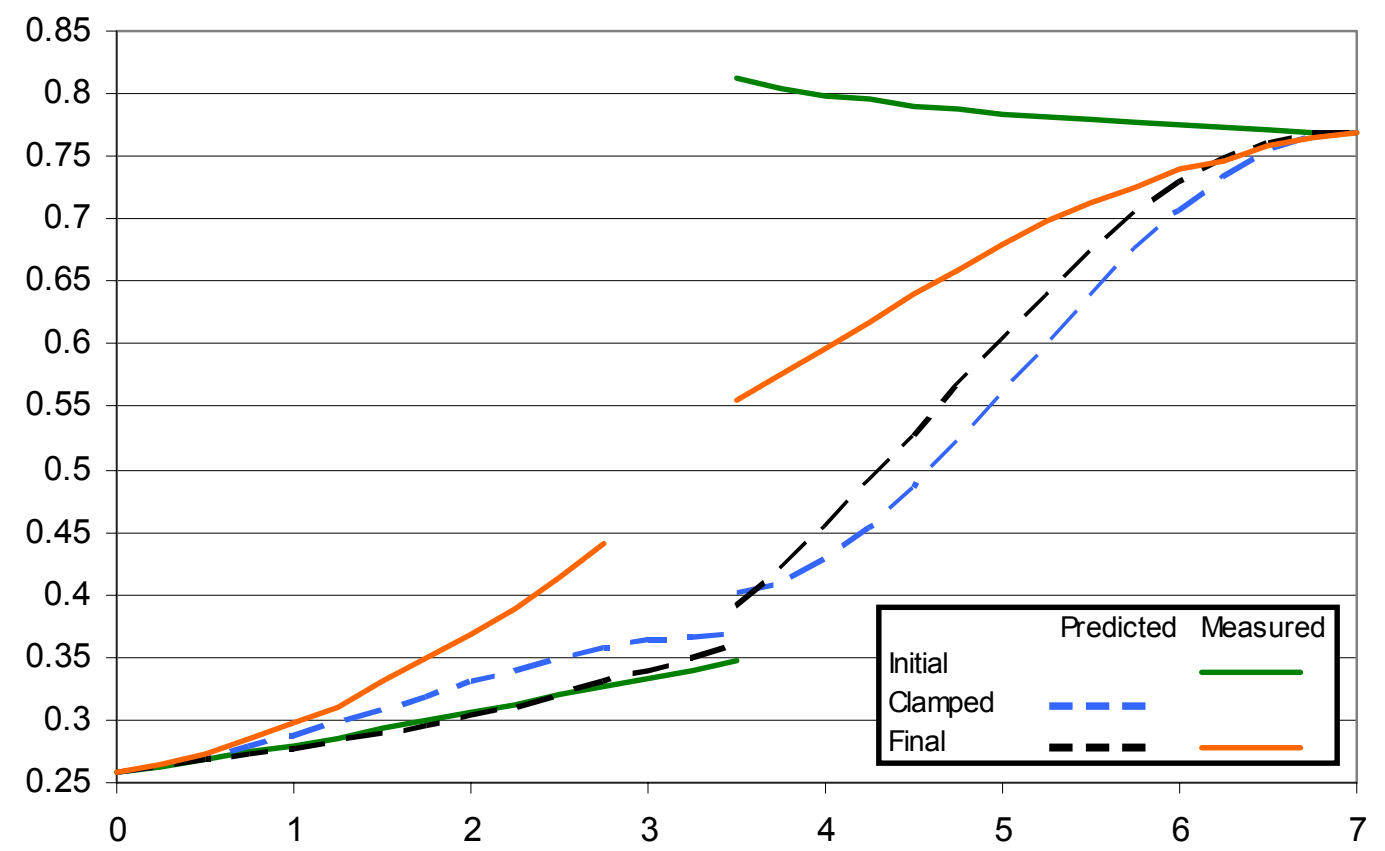

Figure 6-24: Load case 3 cross section results at $x=0.0$

Table 6.7: Load case 3 cross section tabular results at $x=0.0$

\begin{tabular}{|l|c|c|}
\hline & Left Plate & Right Plate \\
\hline Initial Position & 0.347 & 0.812 \\
\hline Clamped Position & 0.370 & 0.402 \\
\hline Offset Ratio / $\mathbf{~}$ & 0.0066 & 0.117 \\
\hline Final Predicted Position & 0.360 & 0.391 \\
\hline Actual Measured Position & $(0.524)$ & 0.556 \\
\hline Absolute Error & $\mathbf{( - 0 . 1 6 4 )}$ & -0.165 \\
\hline
\end{tabular}




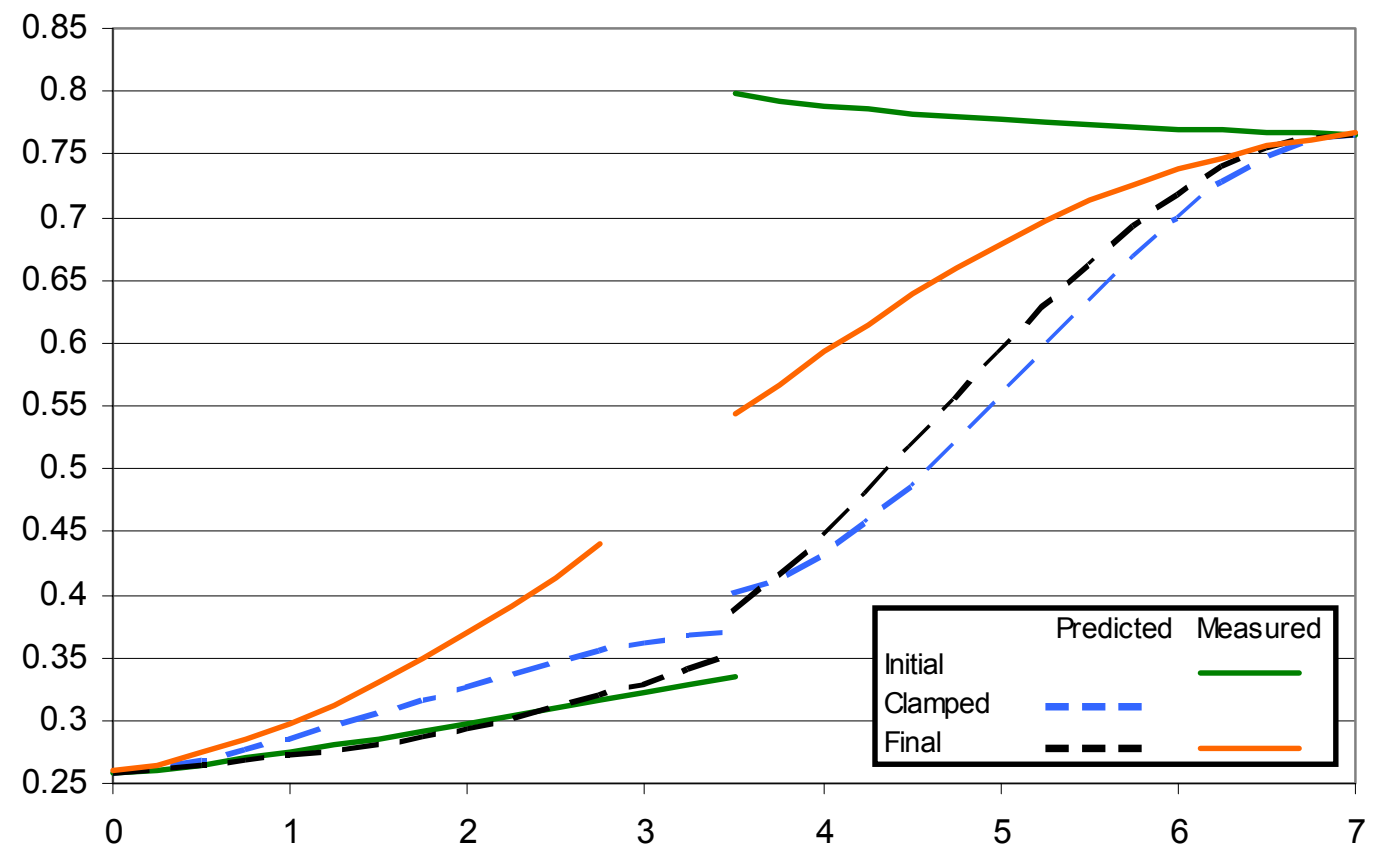

Figure 6-25: Load case 3 cross section results at $x=1.0$

Table 6.8: Load case 3 cross section tabular results at $x=1.0$

\begin{tabular}{|l|c|c|}
\hline & Left Plate & Right Plate \\
\hline Initial Position & 0.335 & 0.798 \\
\hline Clamped Position & 0.370 & 0.402 \\
\hline Offset Ratio $\mathbf{\delta}$ L & 0.01 & 0.113 \\
\hline Final Predicted Position & 0.354 & 0.386 \\
\hline Actual Measured Position & $(0.512)$ & 0.544 \\
\hline Absolute Error & $\mathbf{( - 0 . 1 5 8 )}$ & -0.158 \\
\hline
\end{tabular}




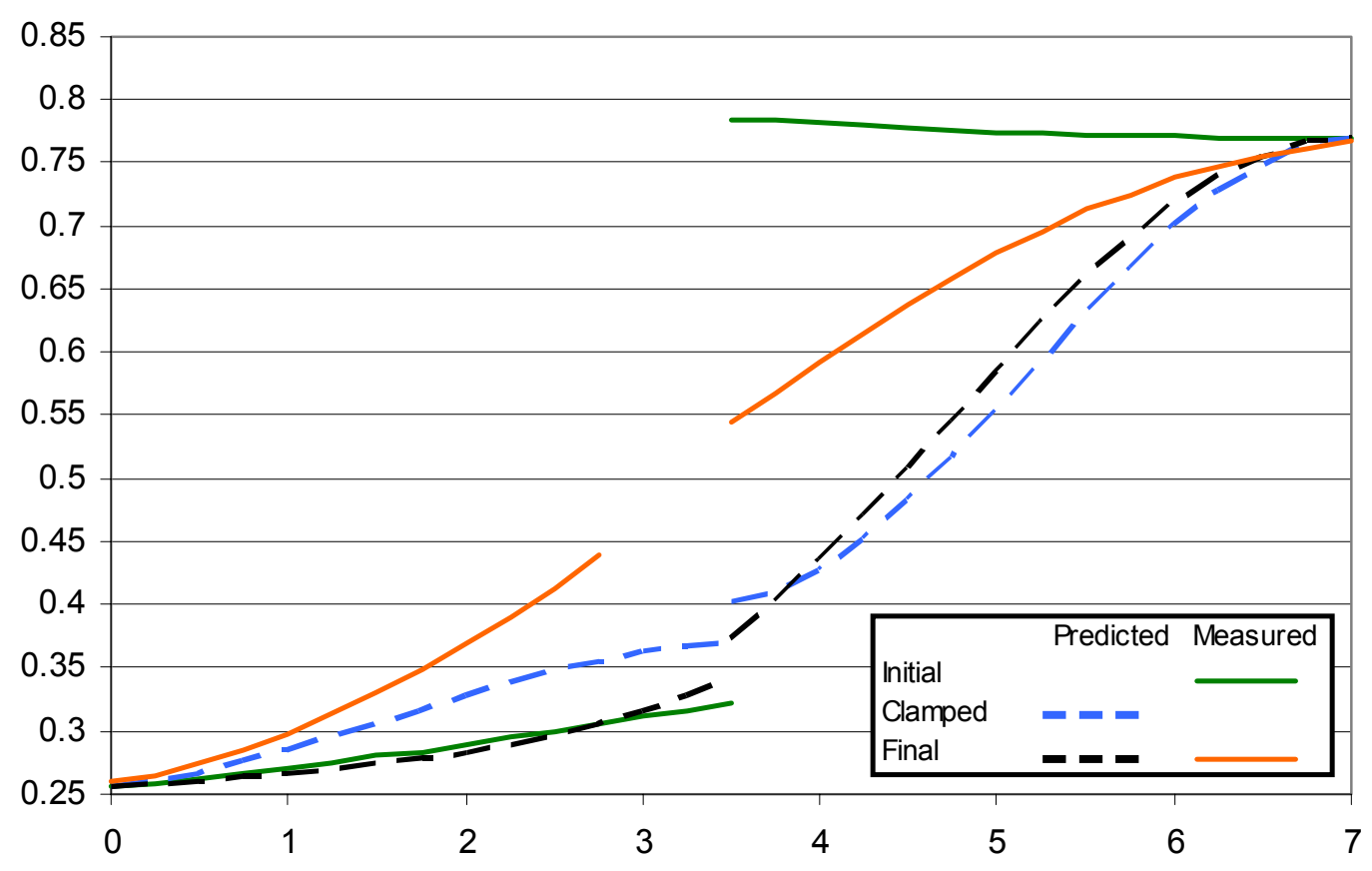

Figure 6-26: Load case 3 cross section results at $x=2.5$

Table 6.9: Load case 3 cross section tabular results at $x=2.5$

\begin{tabular}{|l|c|c|}
\hline & Left Plate & Right Plate \\
\hline Initial Position & 0.323 & 0.785 \\
\hline Clamped Position & 0.370 & 0.402 \\
\hline Offset Ratio $\mathbf{~} \mathbf{L}$ & 0.013 & 0.109 \\
\hline Final Predicted Position & 0.342 & 0.373 \\
\hline Actual Measured Position & $(0.520)$ & 0.544 \\
\hline Absolute Error & $(-0.178)$ & -0.171 \\
\hline
\end{tabular}

From this load case it is clear that with a greater clamping displacement, the PLEA method also increases in inaccuracy. This is a load case which is unlikely in a tightlycontrolled production environment, since the clamping displacements are so great. This load case was analyzed simply to see the PLEA method capability with a wide range of inputs. 
This load case was unique among these load cases, too, in that the lower component was forced upwards toward its assembly position.

\subsection{Discussion of Results}

Due to the small variations being measured, the vertical scales of the graphs were magnified. In some cases, this may make the results look extreme and inaccurate. However, actual offsets were small compared to physical dimensions. The maximum offset ratio tested was 0.117 in load case 3 .

From the above load cases, it is immediately apparent that the first load case, nearest ideal, can be closely approximated by PLEA, while the other two load cases exhibit increasing degrees of inaccuracy. A fundamental assumption of using the PLEA process is that the stiffness matrix is constant throughout each deflection. Due to the large initial clamping deflection in load case 2 and 3 , the predicted final positions were not exact as compared to experimentation. It is thought, however, that in the range of tolerances common to a high-volume manufacturing process, the prediction due to analysis would be much closer to the first load case. It is further believed that with the inclusion of complex, non-linear capabilities of commercial FEA programs, the prediction of the analysis of the second and third load cases may be closer to the expected value.

However, due to the assumed linearity between deflection and force PLEA relies upon for stochastic calculations, this theory will not be verified in this thesis. 


\subsection{Conclusion}

To verify PLEA, simple sheet metal assemblies were analyzed for the final position in three cases of initial offset. By assuming the physical part geometries correspond to the mean statistical solution, the mean PLEA method solution was verified. The methods of assembly, scanning and analysis were detailed. The results found through analysis and experimentation were compared. The verification of PLEA provided valuable insights in the analysis of more complex production parts, as detailed in the next chapter.

The discrepancy in the results could be attributed to the abnormally large initial position error of the parts. It was concluded that any production-quality assembly will be closer to the linear displacement range of the first load case, which predicts the final shape of the analysis fairly well with the appropriate measurement equipment. 


\section{Chapter 7: $\quad$ Boeing Leading Edge Assembly Analysis}

In Chapter 4, the PLEA theory was developed to predict the final shape of fixtured assemblies due to misalignment in the components and assembly fixture. In Chapter 5, the PLEA theory was laid out on process maps. In Chapter 6, experimentation was conducted on a simple lab assembly in an effort to validate the theory. The purpose for the development of the PLEA method has been to predict the assembly of real and complex production parts. With the validated theory, this method can now be used to analyze a real production assembly obtained from the Boeing Company. The completed production assembly is shown in Figure 7-1.
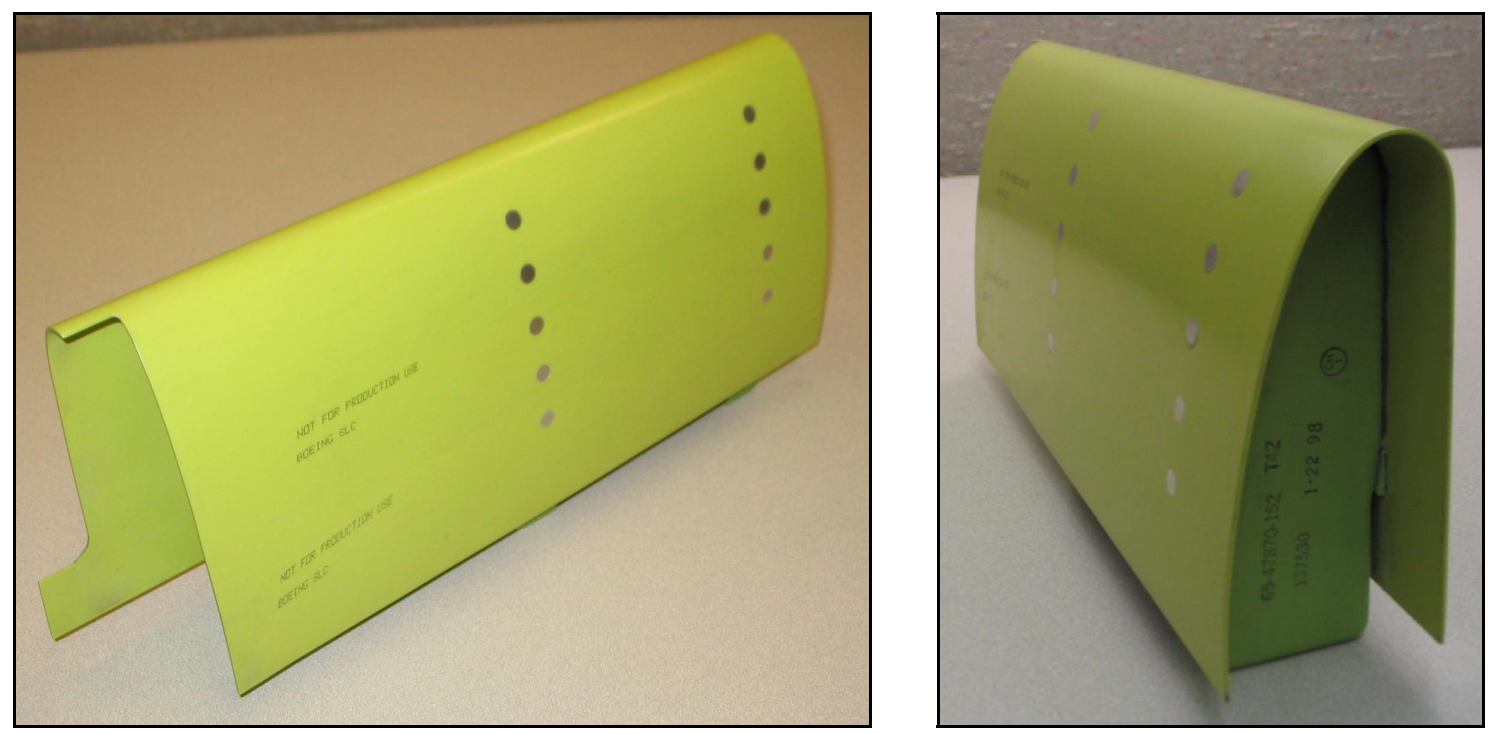

Figure 7-1: Boeing leading edge assembly 


\subsection{Boeing Leading Edge Assembly}

The subassembly constitutes a leading edge flap of a Boeing 737 . Three components comprise the subassembly, two stiff aluminum ribs, which provide structural stability, and a skin of flexible aluminum, which defines the exterior shape of the assembly. To aid in repeatable assembly of these components, an assembly fixture, unique to this assembly process, is used to flex the skin to conform to the ribs prior to the riveting process. The components can be seen in Figure 7-2. The subassembly is approximately 5 " high by 3 " wide by 12 " long. 


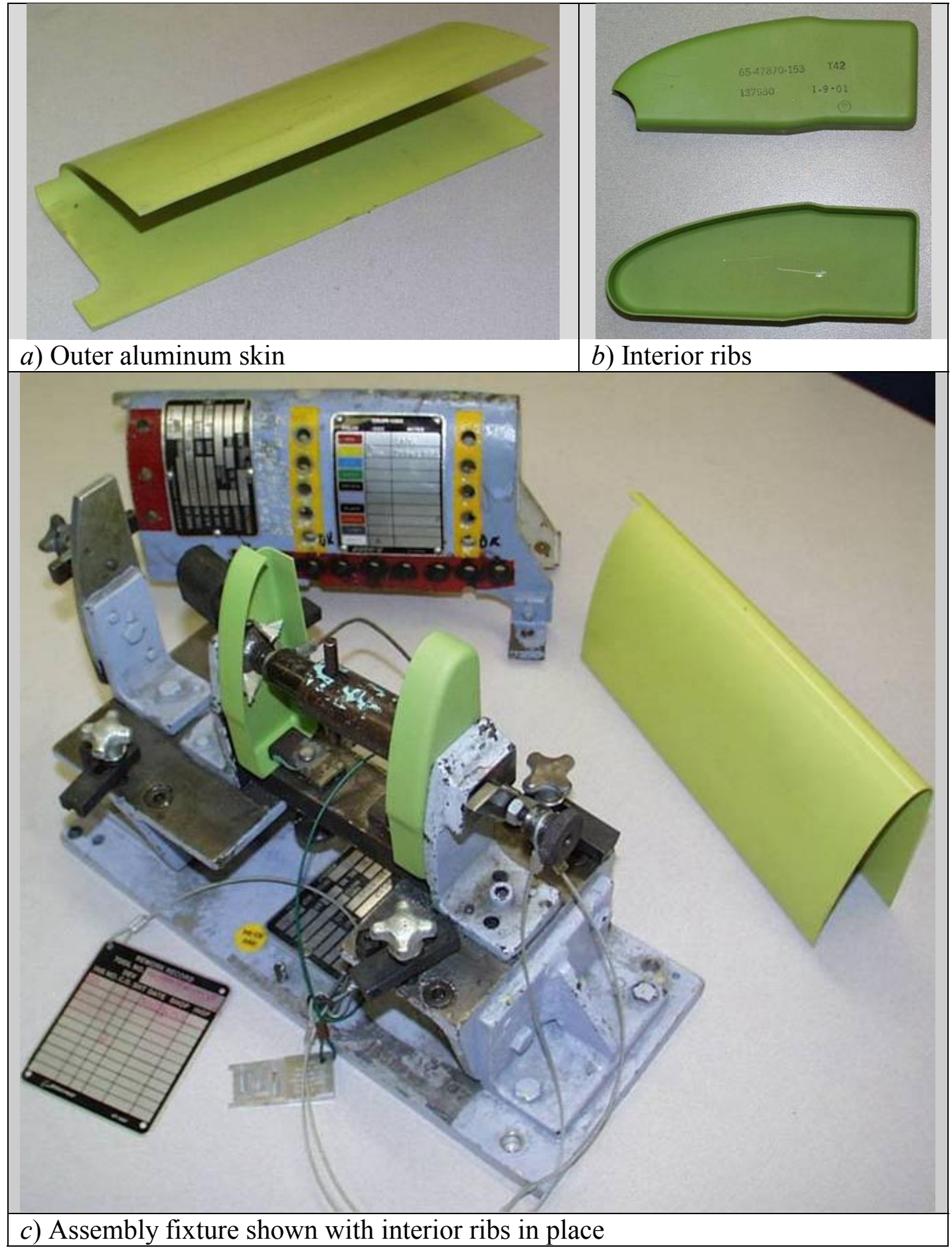

Figure 7-2: Pictures of parts and fixture for leading edge assembly [Mortensen 2002] 
Figure 7-2c shows the ribs located in the fixture and held by the use of a spring-loaded pushrod, while the exterior skin has yet to be located on the fixture. Once the skin is placed over the ribs and located by features native to the fixture, the clamshell is placed over the skin and locked down to the fixture, constraining the skin to its clamped position. The ten holes marked with yellow on the clamshell define the locations for the drilling and riveting of the assembly. Six holes on the clamshell back also define locations for drilling and riveting. Each rib is riveted to the skin in eight places. The assembly production sequence is illustrated in Appendix A.

This subassembly is well-suited for PLEA analysis for three reasons. Firstly, tolerance analysis for this subassembly is vital. The performance of a wing spar is determined by its shape. Tolerances will affect the form of this subassembly, which can adversely affect its function. If the ranges of shapes are known beforehand, the ranges of performances can also be determined and accounted for. Secondly, the ribs and skin are parts of varying shapes and stiffness. This will give us an opportunity to show the robustness of the PLEA analysis method in analyzing different components in an assembly. Thirdly, this subassembly makes use of a fixture to aid in repeatable production. The fixture itself is complex because of its curvature, but can be accurately incorporated into analysis through the use of CMM measurement. 


\subsection{Assembly Modeling}

Using the analysis methods set forth in previous chapters and the modeling techniques learned through simple assembly experimentation, the more complex Boeing leading edge assembly can be analyzed.

\subsubsection{CMM Characterization}

The measuring of the components and fixture was vital to the success of this analysis. Using the advanced contour-measuring capabilities of PCDMIS ${ }^{\circledR}$, the mating surfaces of each rib and the aluminum skin were characterized to 0.001 " resolution. Additionally, the interior surface of the clamshell was also characterized, providing us with the shape of the subassembly in its clamped position. Once the full assembly process was performed on this subassembly by Boeing employees, it was returned for post-assembly CMM characterization.

\subsubsection{Modeling of Components}

Due to the flexibility of the skin and the geometry of the components, assumptions can be made to simplify the analysis without any loss of accuracy. It will be assumed that:

1. The ribs are rigid and minimal rib deflection takes place during the clamping stage. This allows us to analyze only the flexible skin in the clamping stage. This also reduces the springback forces to those of the skin only, reducing the complexity of this analysis 
2. The clamshell fixture provides minimal contact with the skin in the clamping stage. While it is possible to model the clamping stage as a specified displacement on each node individually, such an exhaustive loading scheme is not practical nor does it resemble real assembly techniques, and might lead to analysis instability. It will be assumed contact with the clamshell fixture will occur only at the edges of the skin, on the flat surface of the skin and at the rivet points.

3. The modeling of adhesives and sealants were neglected in our analysis. This is because, even when properly cured, these substances impart no structural rigidity to the assembly. A thin layer of sealant fills the voids between the ribs and the skin, but is displaced with the installation of the rivet. It is further assumed that the sealant creates a decreased chance for mid-node contact, which will be helpful in future work when the possibility of mid-node contact will be studied.

\subsubsection{Skin Modeling}

The skin was modeled as flexible Shell63 elements. From the CMM scans, and similar to the process put forth in the previous chapter, the scan points were mapped into points in an orthogonal coordinate system native to the fixture base. Care was taken to ensure each point on the curved surface was about 0.2 " from its neighbors in all directions, and that any flat element does not span more than $15^{\circ}$ of an arc, a caution given in Mortensen [2002]. The result is a skin with 2812 nodes and 2704 elements, as seen in Figure 7-3 as modeled in ANSYS ${ }^{\circledR}$. 


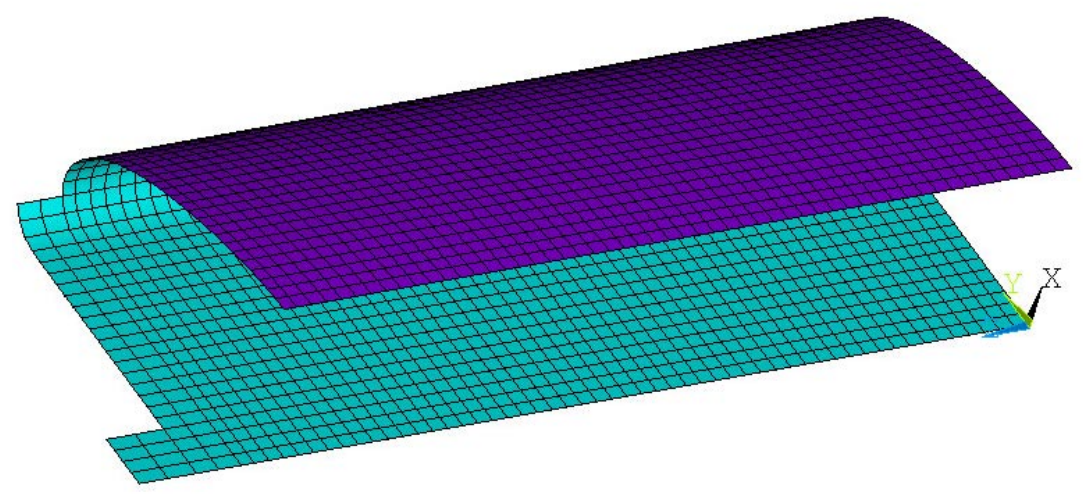

Figure 7-3: ANSYS $^{\circledR}$ models of leading edge skin

\subsubsection{Rib Modeling}

The ribs are likewise modeled with Shell63 elements. Rigidity is imparted to each rib by the flanges on the edges of the part. The nodes were likewise aligned to measured closure positions. The notched rib stiffener has 453 nodes and 419 elements, while the unnotched rib stiffener has 484 nodes and 453 elements. Both ribs can be seen in Figure 7-4 as modeled in ANSYS ${ }^{\circledR}$. 

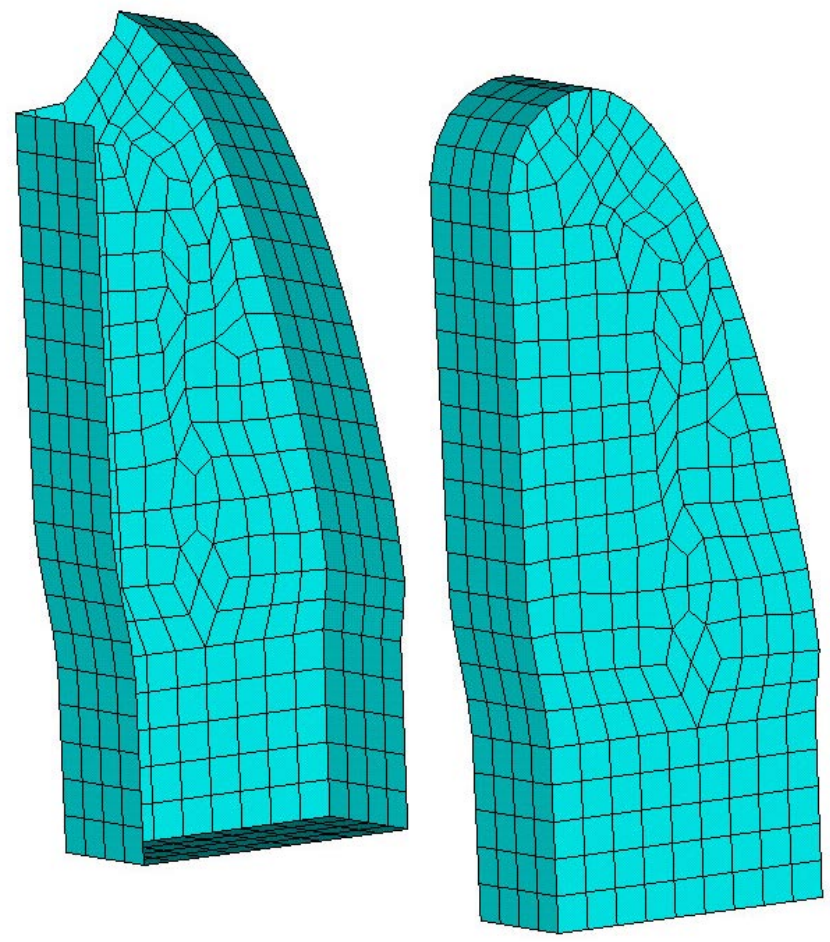

Figure 7-4: ANSYS ${ }^{\circledR}$ models of the leading edge rib stiffeners

\subsection{Assembly Analysis}

With the skin and the ribs modeled in ANSYS ${ }^{\circledR}$, the assembly can be analyzed using the PLEA methods.

\subsubsection{Clamping Analysis}

Due to the rigidity of the ribs, they were not included in the clamping stage analysis. The skin was assigned closure constraints equal to those found by scanning the holes and inner surface of the clamshell fixture on the CMM and determining the intersection of the centerline of the holes and the inner surface. 
This was not an easy task. It was difficult to determine the actual locations of the closure nodes on the undeflected skin, since they are drilled after the parts are clamped in the fixture. By using the CMM scans of the fixture and including normal offsets, the clamped shape of the skin was estimated. After the skin closure nodes were located, the distances between neighboring rib nodes were determined. These values were used to find the distances between the closure nodes on the undeflected skin. While the straight section was easy to find from part drawings, this method was very useful for the curved section of the skin. Inherent in this assumption is that the skin does not stretch between closure nodes. Because the holes were drilled after the skin was positioned in the fixture, this was a valid assumption. 


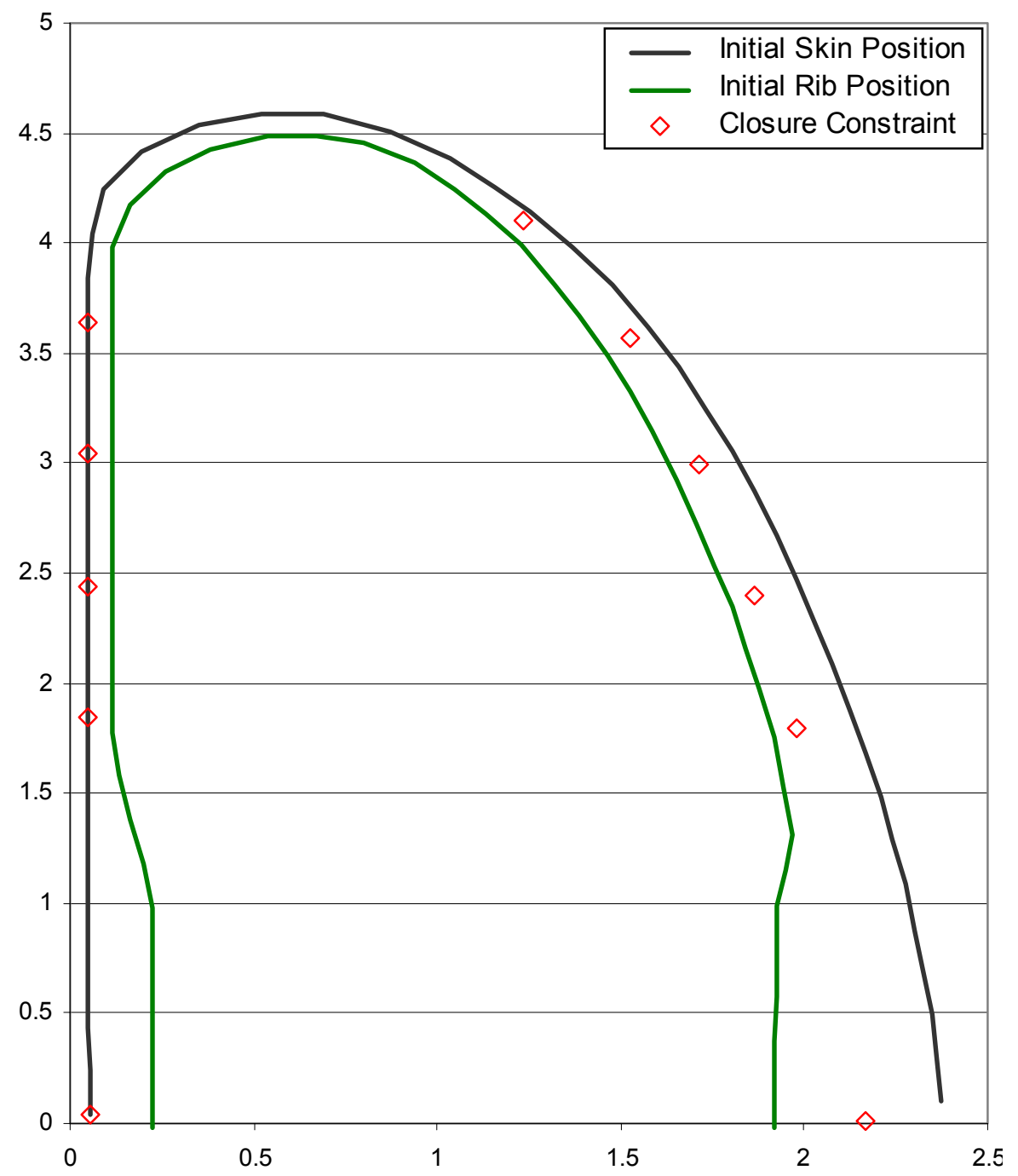

Figure 7-5: Boeing Assembly Cross-section showing Rib, Skin and Fixture Points

Two types of constraints were defined in the ANSYS ${ }^{\circledR}$ model. A fixed constraint was used on the closure nodes of the flat section of the skin, as well as the edge of the skin where it contacts the fixture base. These constraints are fixed from motion in the $x$ direction, normal to the flat portions of the rib and fixture, and rotation about the $z$-axis. A moving constraint was used on the closure nodes of the curved section, because it is expected that these nodes will translate. These closure nodes are only located at the cross sections $x=1.4$ and $x=5.6$, and correspond to the riveting positions. An additional 
closure constraint was applied to the edge of the curved section of the part, as it too was clamped against the fixture. This closure constraint was applied on each edge node on the lower edge. Even though the moving constraints can have 3 constraints (translation in the $x$ - and $y$-directions and rotation about the $z$-axis), the moving constraints only had 2 of these conditions applied to avoid over-constraint. In our case, only translation in the $x$ direction and rotation about the $z$-axis were considered. Failure to exactly constrain the model leads to surprising results, shown in Figure 7-6.

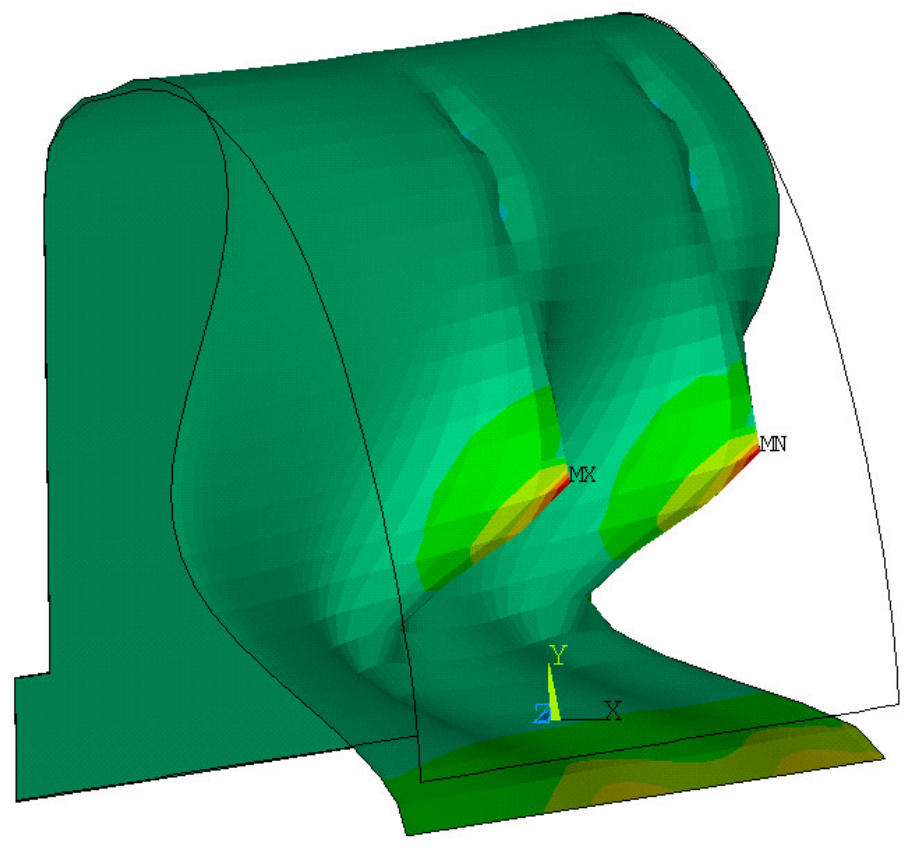

Figure 7-6: Inaccurate clamping results due to overconstraint

The results from the successful analysis of the clamping stage are given in Figure 7-7 and Figure 7-8. 


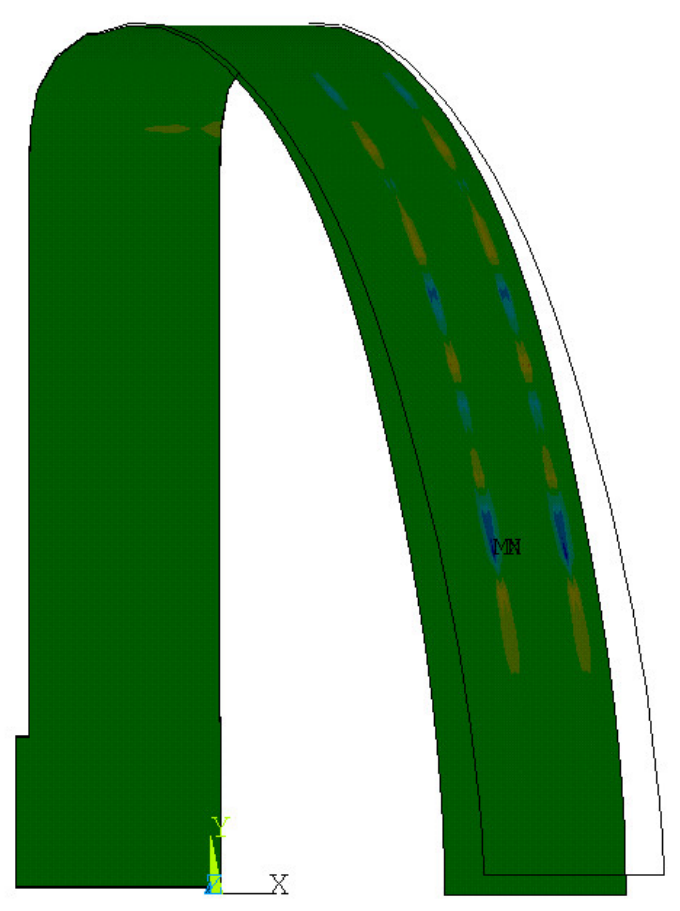

Figure 7-7: Initial and clamped shape of skin 


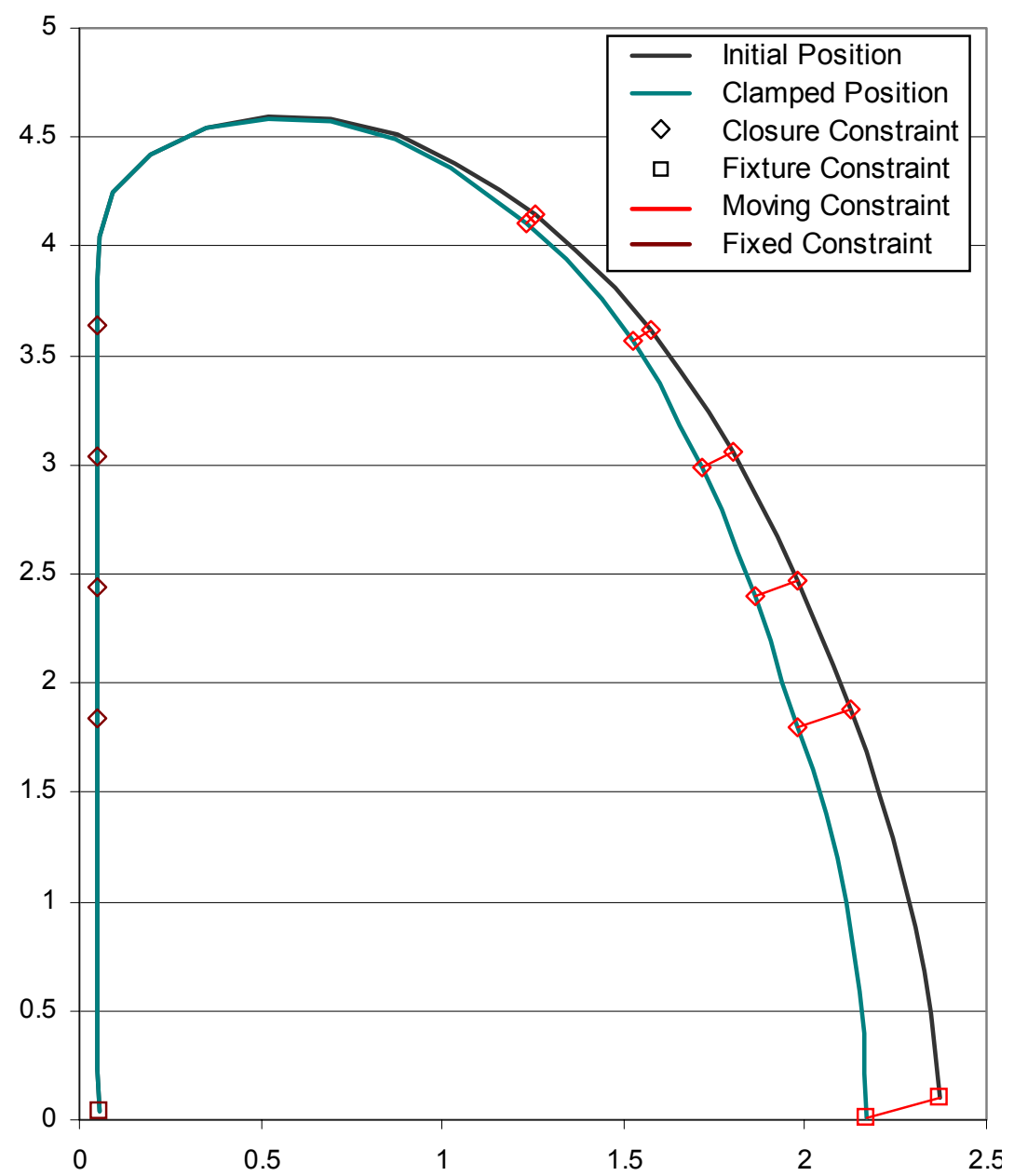

Figure 7-8: One cross-section illustrating fixture constraints and skin positions

\subsubsection{Springback Analysis}

The deformed shape of the skin and the assembly forces are taken from the clamping analysis results. These are used as inputs in the springback analysis. The reverse assembly loads are applied to the closure nodes on the skin, which are also constrained in all degrees of freedom to neighboring nodes on the ribs, simulating a rivet. The fixture constraints also have reverse assembly loads applied, but do not have degree of freedom constraints applied. This simulates the reactions arising from the assembly being completely removed from the fixture. 


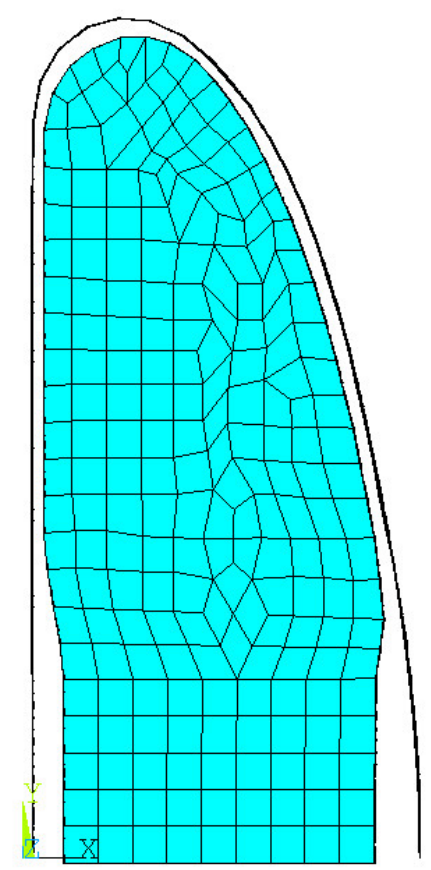

Figure 7-9: Ribs and skin modeled for springback analysis

Because it was assumed in this stage the part was fully removed from the fixture, any nodes on the skin cannot be used to constrain the assembly from rigid-body motion. It will be assumed, since the fixture is no longer in contact with the skin, the whole skin should be free from applied constraints. Therefore, to constrain the assembly from rigidbody motion in the springback stage, motion constraints were applied to the base flange of the ribs. These features were chosen for DOF constraint because they are not anticipated to deform during the springback analysis. 


\subsection{Analysis Results}

After the analysis was run, cross sections were plotted and compared to the actual assembly.

\subsubsection{Prediction Summary}

The cross section at $x=5.6$ corresponds to the riveting locations of the skin to the notched rib stiffener. Figure 7-10 illustrates the initial, predicted and actual result at that cross section. All other cross sections also show general agreement.

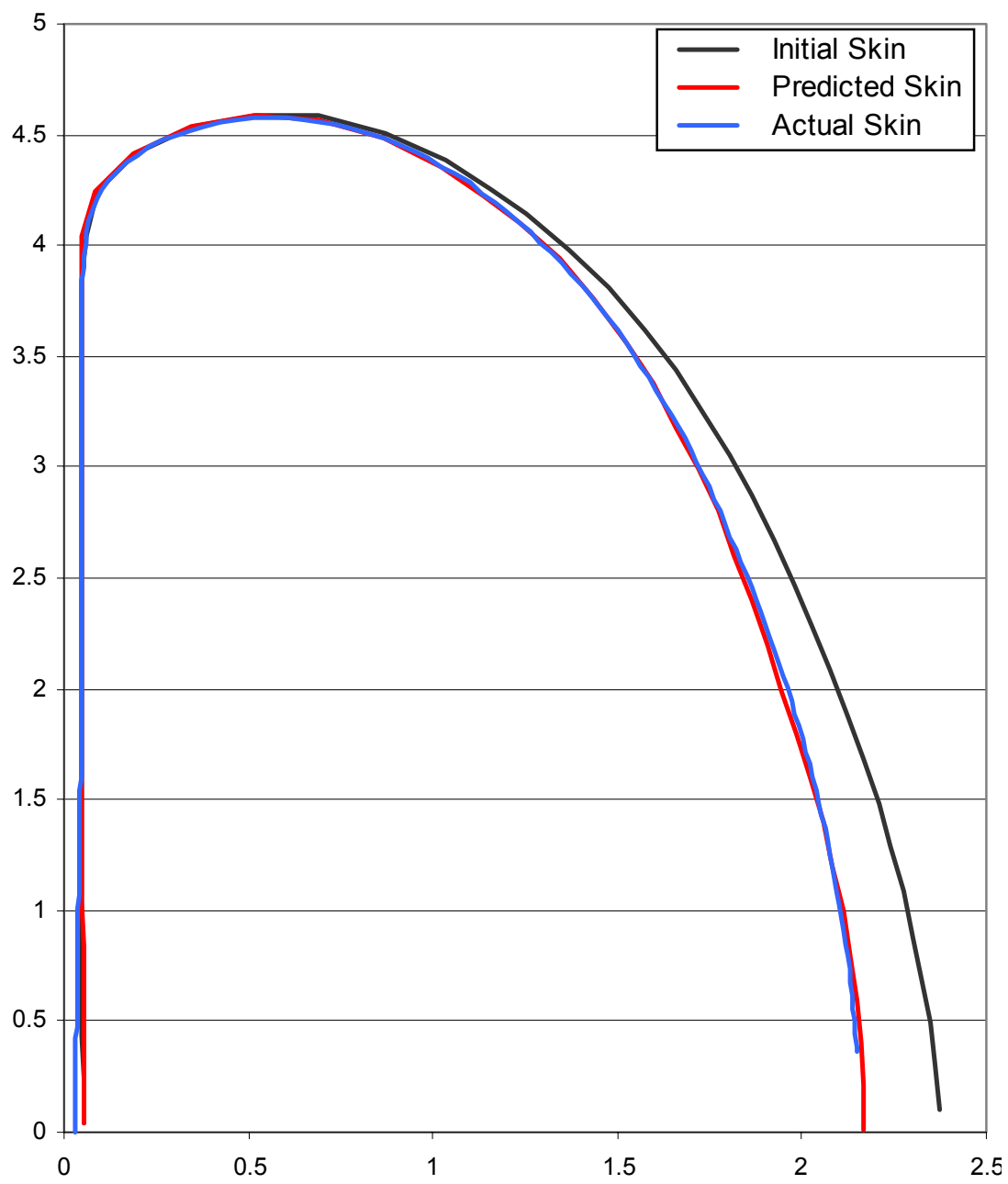

Figure 7-10: Skin analysis results at $\boldsymbol{x}=\mathbf{5 . 6}$ 
To test the PLEA process, four nodes of interest on this cross section were chosen. These nodes consist of each unsupported edge of the skin, a rivet location and a location between rivets. These nodal locations can be seen on Figure 7-11.

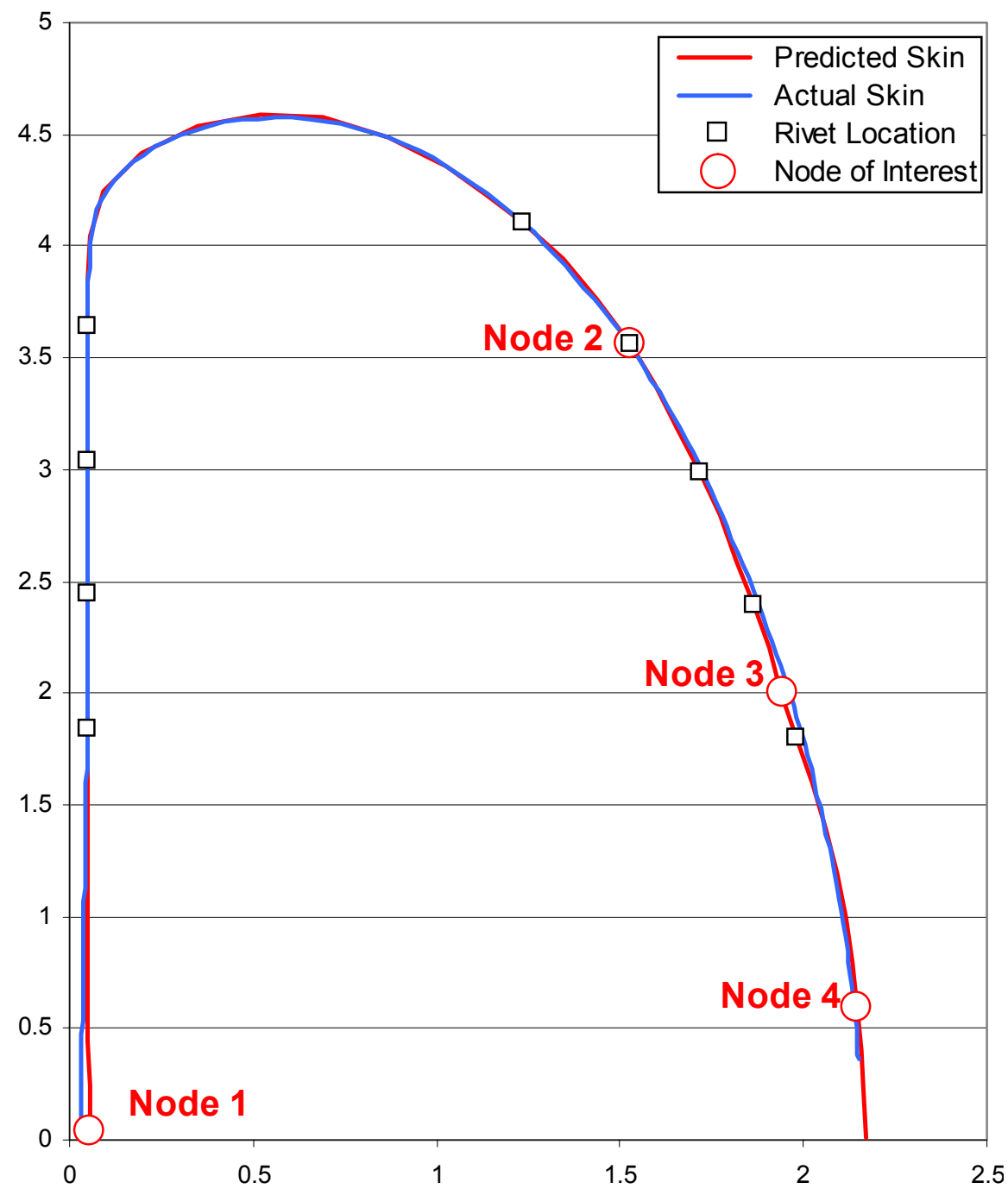

Figure 7-11: Nodes of interest for quantitative comparison

Because these points have different initial deflections, an absolute plot of distances between the initial and final results will be presented. These results are presented in Table 7.1. The first column corresponds to the absolute distance between the initial nodal 
location and the actual nodal location. The second column corresponds to the absolute distance between the initial nodal location and the nodal location as predicted by PLEA. The third column is the difference between the first and second column, and corresponds to the absolute error.

Table 7.1: Nodal deflection results

\begin{tabular}{|l|c|c|c|}
\hline & $\begin{array}{c}\text { Distance between initial } \\
\text { and actual locations }\end{array}$ & $\begin{array}{c}\text { Distance between initial } \\
\text { and predicted locations }\end{array}$ & $\begin{array}{c}\text { Absolute } \\
\text { error }\end{array}$ \\
\hline Node 1 & 0.048 & 0.001 & 0.047 \\
\hline Node 2 & 0.074 & 0.072 & 0.002 \\
\hline Node 3 & 0.141 & 0.158 & -0.017 \\
\hline Node 4 & 0.211 & 0.202 & 0.009 \\
\hline
\end{tabular}

From the small error results given in Table 7.1, the PLEA method provides good results for all nodes of interest. A maximum absolute error of 0.047 inches was observed at node 1 .

\subsubsection{Fixturing Effects}

Another correct prediction of the PLEA method is that the further away from the rib stiffeners the cross section, the more the skin exhibits an "alligatoring" effect, where the edges of the skin tend to translate in the $x$-direction, away from each other, when removed from the fixture. This can be seen in the contour plot of Figure 7-12. 


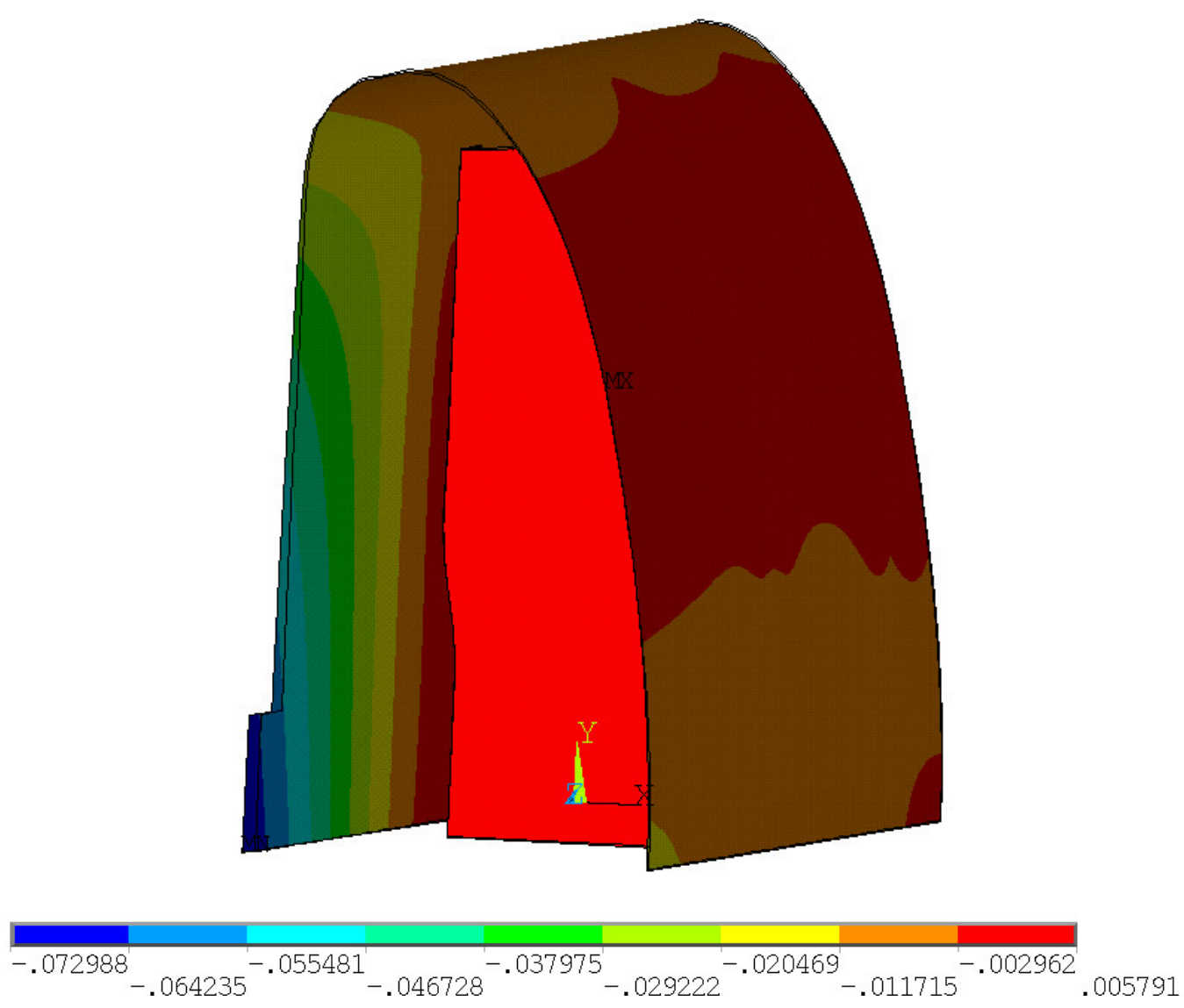

Figure 7-12: Assembly plotted with $x$-displacement contours

Figure 7-14 quantitatively illustrates the "alligatoring", or spreading effect of the skin edges. It is a plot of the clamped and final positions of each point on the lower edge of the straight section of the subassembly, as indicated in Figure 7-13. The "alligatoring" effect is most visible on the edges away from the rigid ribs. 


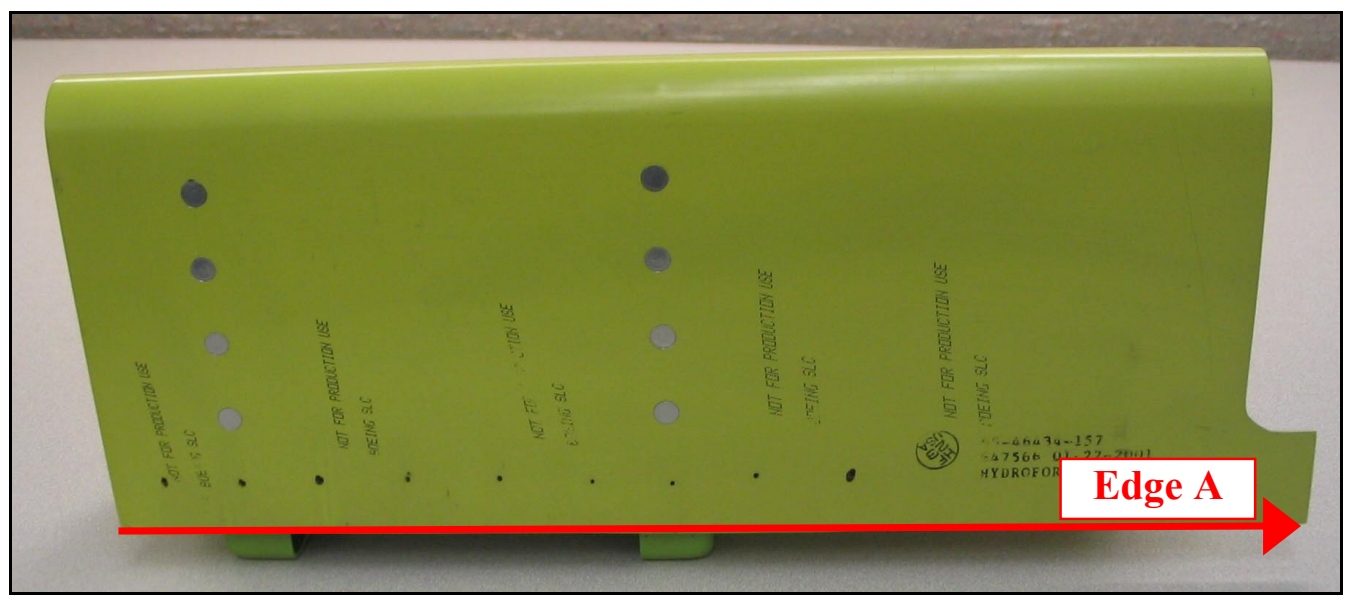

Figure 7-13: Coordinate axis for Figure 7-14

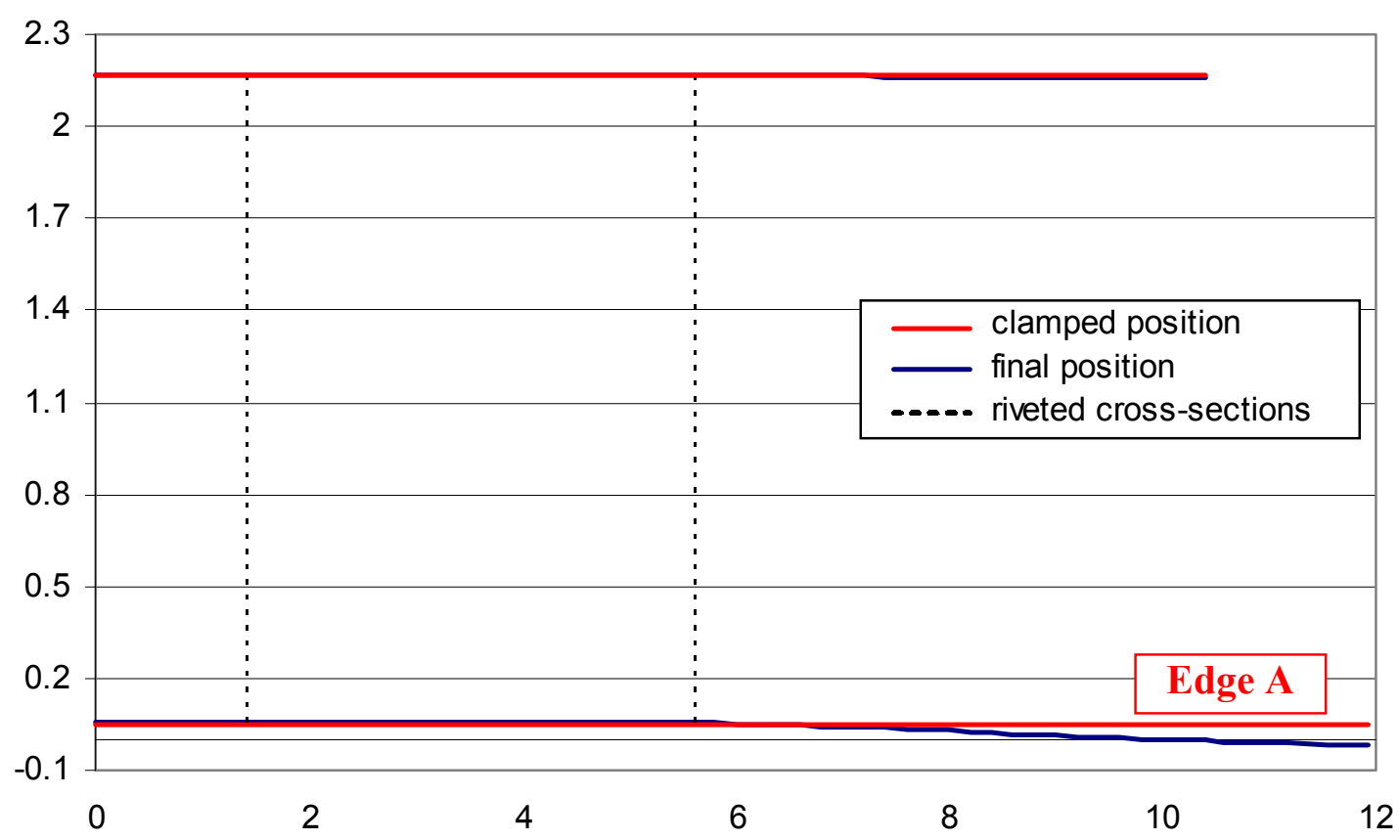

Figure 7-14: "Alligatoring" effect as viewed from assembly top 


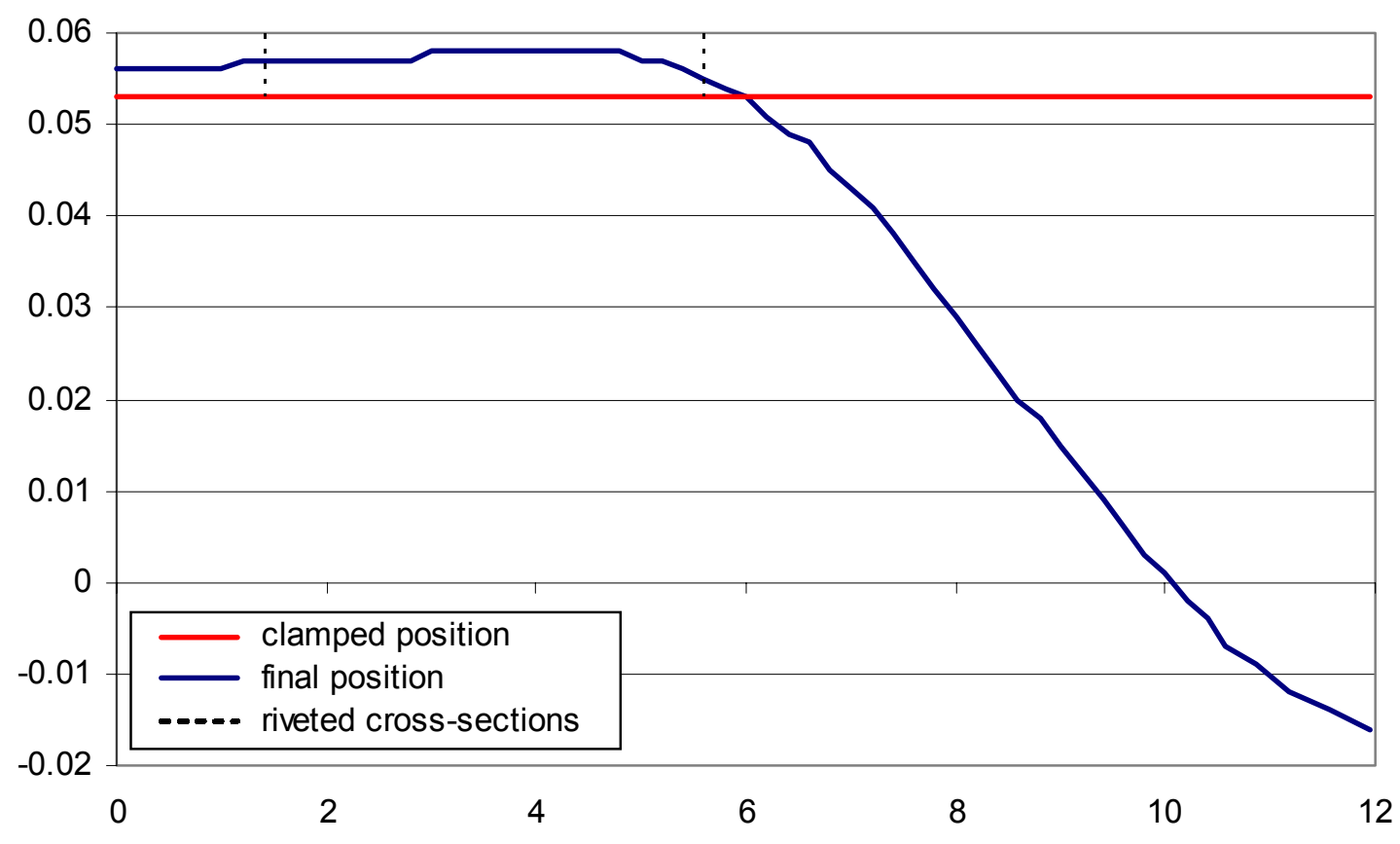

Figure 7-15: Magnified deformation plot illustrating "alligatoring" of Edge A

\subsection{Discussion of Results}

Using PLEA methods to analyze this production assembly has shown good agreement with the final assembly shape. These results validate PLEA and show it can be used to analyze complex assemblies and provide a good estimate of nonlinear effects through linear superposition.

Two further effects can be tested in later research. Because only one assembly was obtained from Boeing, statistical variation could not be determined. With more assemblies obtained from Boeing and analyzed, the mean and variance of the assembly can be determined. Furthermore, with mounted strain gages, the stress solution can be verified. A caveat which comes with the addition of strain gages is that their positioning could potentially make assembly more difficult. Care should be taken to mount them at 
locations of interest where they will not interfere with any aspect of the assembly process.

When the statistical solution is determined for the assembly, the information can be used to make improvements to the process. The mean solution predicts the average distortion of the assembled parts. If modified distortion is desired, the part or the fixture can be modified to partially compensate for the mean distortion solution. These changes can be simulated in PLEA quickly to determine the appropriate modifications by simply changing the mean displacement loads. The solution for the variance predicts the expected statistical quality of the post-assembly shape. To tighten the statistical spread of the assembly, tooling changes would need to address the surface waviness and individual tolerances of the compliant parts.

\subsection{Conclusions}

In this chapter, a real production assembly was analyzed using the developed PLEA method. Because only a single assembly was obtained, only the mean PLEA solution was determined. The mean post-assembly surface was determined and compared to actual assembly measurements. The analysis and actual assembly agreed well. This analysis proves PLEA can be implemented on a real production assembly. The two most difficult PLEA modeling tasks were to determine the proper loading in the clamping stage and defining the closure nodes on the skin, which underwent multidirectional clamping deflection. However, once these things are determined, PLEA can be applied to the assembly with successful and accurate results. 


\section{Chapter 8: Conclusions and Recommendations}

The main objective of this thesis was to develop a comprehensive method for the statistical tolerance analysis of flexible assemblies which were assembled using rigid fixtures and supports. This objective is accomplished with the realization of two subobjectives.

1. Development of a new method which incorporates rigid fixture PCFR effects into existing flexible part tolerance analysis methods.

2. Application of the new method on both research lab and production-quality assemblies to assess method capability.

These sub-objectives have been met and have shown the new method, PLEA, to be a satisfactory method of applying tolerance analysis to fixtured assemblies. The significant findings of the PLEA process are presented. The strengths and limitations of the PLEA process are presented as well as my contributions and suggestions for future research.

\subsection{Conclusions}

Because the FASTA method is unable to account for the fixturing of compliant parts prior to assembly, a new method needed to be developed which could account for rigid fixture positions. This new method, PLEA, was developed and demonstrated to be an 
effective statistical tolerance analysis method for flexible assemblies typical to aerospace structures. The major research findings are summarized below.

\subsubsection{Piecewise-Linear Elastic Analysis Method}

\subsubsection{The Piecewise-Linear Elastic Analysis Theory}

To analyze flexible assemblies in fixtured PCFR cycles, PLEA theory was first developed and tested analytically.

Important findings include:

- PLEA properly accounts for nonlinear fixtured assembly processes with the superposition of linear FEA analyses.

- PLEA demonstrated the effects of different clamping locations on springback, whereas FASTA assumes final shape is independent of clamp location.

- PLEA accounts for arch effects due to slip joint elongation by recalculating the stiffness of the components in their deflected position in the fastening stage of the analysis.

- PLEA can account for in-plane stiffness, which could be important for the proper analysis of the release and springback effects in certain cases.

\subsubsection{PLEA verification}

Verifying the PLEA theory on simple, lab assemblies yielded encouraging initial results. Important findings include: 
- Applied clamping loads must imitate actual loading conditions to avoid overconstraint and model instability.

- Due to the limitations in linear FEA, analysis yields accurate results if the gaps between the parts and the fixture are within the range of tolerances of the parts.

\subsubsection{Application of PLEA to a real production assembly}

\subsubsection{Assembly Modeling}

The application of PLEA to actual production parts with complex clamping conditions yielded information vital to making PLEA more robust.

Important findings include:

- To avoid unintentional overconstraint, 3-D loads must be modeled with at least 1-D of allowance for adjustment.

- The fixture should be reduced in complexity by limiting the clamping loads to the supports and the mating surfaces.

\subsubsection{Assembly Solutions}

PLEA produced accurate results when the analytical and physical assemblies were compared for overall deflection.

Important findings include:

- PLEA is able to correctly model springback effects such as "alligatoring".

- PLEA yielded excellent agreement with a production assembly. 
- PLEA has been verified on the mean solution of a typical aerospace structure and is a viable method to performing fixtured compliant assembly analysis.

\subsubsection{Statistical Tolerance Analysis Flowchart}

To benefit the analyst, a statistical tolerance analysis flowchart was developed. This flowchart, Figure 8-1, shows the design decisions to make in order to select the proper analysis method. Once the method is selected, references to aid in the application of the method are listed.

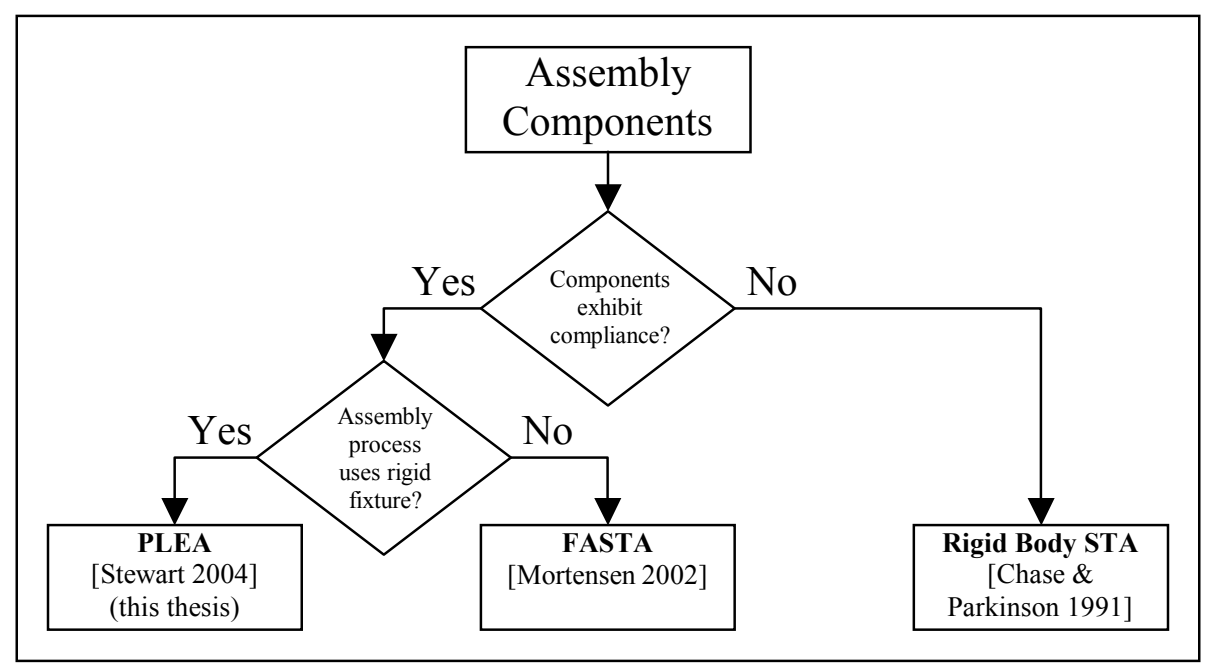

Figure 8-1: Statistical tolerance analysis method selection 


\subsection{Contributions}

The significant contributions made in this thesis are:

- Development and presentation of Piecewise-Linear Elastic Analysis (PLEA) method for analyzing the tolerance analysis of compliant assemblies

- Investigation of PLEA method accuracy with increasing initial offset from fixture

- Detailing of modeling and loading conditions for successful PLEA

- Verification of the PLEA method using both laboratory and production assemblies

- Development of the detailed layout for a full implementation of the PLEA process

- Validation of the actual part data and data analysis flowchart

- Detailing of the Boeing assembly process, as demonstrated by trained Boeing employees

\subsection{Suggested Future Research}

Though the PLEA method has been developed and analyzed, for further implementation of this analysis method in production cycles the following needs should be addressed:

\subsubsection{Verify Stochastic Solutions}

Although the PLEA method was developed to apply to populations of assemblies, feasibility limited the verification of the method to one assembly in this thesis. By 
obtaining and analyzing a population of production assemblies, the stochastic assembly solutions can be verified readily using the developed theory. Unlike most stochastic solutions, however, the stress covariance cannot be solved element-by-element, due to element interactions, as shown by Bihlmaier [1999]. Methods to solve for the stress covariance in each analytical stage of the PLEA process should be studied further.

\subsubsection{Inclusion of Mid-node Contact and Inelastic Deformations}

In this thesis, it was assumed no mid-node contact would occur between the rivets during clamping to the fixture or fastening mating surfaces. It was also assumed that the parts exhibit perfect elasticity. With the incremental abilities of FEA software packages, nonlinear analysis is possible, but cannot be done without changing the stiffness matrix of the parts incrementally. By updating the stiffness matrix, a linear relationship is not maintained. A nonlinear relationship between $F$ and $\delta$ means the relationship between $\Sigma_{F}$ and $\Sigma_{\delta}$ cannot be defined by the covariant equation given by Equation 3-31. Midnode contact will greatly complicate the statistical solution, since the locations of midnode contacts are unknown and random. Exploring this new relationship will extend the PLEA to analyze assemblies with associated nonlinearities.

\subsubsection{Surface Waviness Characteristic Sensitivity Analysis}

The PLEA method, like FASTA, relies on real component data in order to calculate the gap covariance. In addition to the translational, rotational and surface warping specifications needed for gap characterization illustrated in Mortensen [2002], surface 
waviness data is needed as shown by Tonks [2002]. This surface characteristic data includes information pertinent to covariance calculation, such as the spatial frequency spectrum or polynomial series information. Actual scanned surface data is preferred, but this surface characteristic data is not known before production. For statistical tolerance analysis, it is common practice to substitute data from similar processes or prior designs at the design stage. Such data must be included in the analysis before parts are made to guarantee the proper statistical characterization of the assembly. There is a need to investigate surface characteristic sensitivity in the design stage. The question which must be answered is: "Can a few discrete points on the frequency spectrum be estimated from prior designs or previous process experience?" A study of the sensitivity to frequency and amplitude error is needed in order for a designer to specify probable surface characteristics so that both FASTA and PLEA may be used with the degree of error known ahead of time.

\subsubsection{Commercial Software Development}

The eventual goal of this research is the development of software made available to the designers who would benefit from its analytical capabilities. To accomplish this, PLEA must be incorporated into commercial software in a user-friendly environment, requiring little user intervention. The software must have two key abilities. It must be able to manipulate and organize information obtained from a commercial FEA program. Such information would include the nodal stress solutions found through analysis as well as the global stiffness, constitutive and kinematic matrices necessary for a covariant solution. Additionally, the software must support memory-intensive calculations, such as large 
matrix inversions and matrix storage. There is an additional, but inherent need for this information to be displayed graphically by the PLEA software or the FEA program, in an effective format, so the designer can then make design decisions using the data. By using the process flowcharts and research-quality codes, both from previous works and from this thesis, the PLEA and FASTA methods may be programmed into useful design software tools.

\subsection{Closing Statement}

Statistical tolerance analysis is a valuable tool for the designer desiring to control assembly variability. PLEA extends this powerful technique of statistical tolerance analysis to compliant assemblies. It has great potential in industries manufacturing precise, yet flexible assemblies. The methods developed and presented in this thesis, when fully researched and developed into a commercial-quality package, will help the designer to lower production costs and improve quality of compliant assemblies. With ever-decreasing costs of computer speed and memory storage, as well as ever-increasing emphasis on variation control and the reduction of scrap and rework, PLEA can and should be applied to assemblies of all ranges of complexity in a broad range of industries, including aerospace, automotive, electronic and domestic products. 


\section{References}

Bihlmaier, B. 1999. Tolerance Analysis of Flexible Assemblies Using Finite Element and Spectral Analysis. M.S. Thesis, Brigham Young University, Provo, Utah.

Camelio, J., S. J. Hu and D. Ceglarek, 2001, “Modeling Propagation of Multi-Station Assembly Systems with Compliant Parts”. Proceedings of the ASME 2001 Design Engineering Technical Conferences and Computers and Information in Engineering Conference, Pittsburgh, PA. Sep. 9-12, 2001.

Camelio, J. A. 2002. Modeling and Diagnosis of Dimensional Variation for Assembly Systems with Compliant Parts. Ph.D. Dissertation, University of Michigan, Ann Arbor, Michigan.

Camelio, J., S. J. Hu and D. Ceglarek, 2002, “Impact of Fixture Design on Sheet Metal Assembly Variation". Proceedings of the ASME 2002 Design Engineering Technical Conferences and Computers and Information in Engineering Conference, Montreal, PQ, Canada. Sep. 29- October 2, 2002.

Camelio, J., R. Webbink, S. J. Hu, K. Iyer, 2003, "Recent Advances in Compliant Assembly Variation Analysis". Managing Geometric Uncertainty in the Product 
Lifecycle: The $8^{\text {th }}$ CIRP International Seminar on Computer Aided Tolerancing.

Charlotte, North Carolina. April 28-29, 2003.

Chang, M. 1996. Modeling the Assembly of Compliant, Non-ideal Parts. Ph.D.

Dissertation, Massachusetts Institute of Technology, Cambridge, Massachusetts.

Chang, M. and D. C. Gossard, 1997, "Modeling the Assembly of Compliant, Non-ideal parts", Computer-Aided Design, Vol. 29, No. 10, pp. 701-708.

Chang, M., J. Kim, H. Rho and S. Ha, 1999, "Representation of Assembly and Inspection Processes for the Tolerance Analysis of Automobile Assemblies" Global Consistency of Tolerances: Proceedings of the $6^{\text {th }}$ CIRP International Seminar on Computer-Aided Tolerancing. Enschede, Netherlands: pp. $223-230$.

Chase, K. W. and A. Parkinson. 1991. "A Survey of Research in the Application of Tolerance Analysis to the Design of Mechanical Assemblies". Research in Engineering Design 3 (January): pp. $23-27$

Crisfield, M. A. 1986. Finite Elements and Solution Procedures for Structural Analysis. Swansea, UK: Pineridge Press Limited. 
Cvetko, R., K. W. Chase and S. P. Magleby, "New Metrics for Evaluating Monte Carlo Tolerance Analysis of Assemblies", Proceedings of the ASME International Mechanical Engineering Conference and Exposition, Anaheim, CA. Nov. 15-20, 1998.

Dahlström, S., S. J. Hu, and R. Söderberg, “Identfying Variable Effects on the Dimensional Quality of Compliant Assembly, Using Computer Experiments". Proceedings of the ASME 2002 Design Engineering Technical Conferences and Computers and Information in Engineering Conference, Montreal, PQ, Canada. Sep. 29October 2, 2002.

Gao, J., K. W. Chase and S. P. Magleby, "Comparison of Assembly Tolerance Analysis by the Direct Linearization and Modified Monte Carlo Simulation Methods". Proceedings of the ASME Design Engineering Technical Conferences, Boston, MA, 1995, pp.353-360.

Glancy, C. and K. W. Chase, “A Second-order Method for Assembly Tolerance Analysis" Proceedings of DETC99: 1999 ASME Design Engineering Technical Conference, Las Vegas, NV. 8 pages.

Hochmuth, R., H. Meerkamm and W. Schweiger, 1998. "An approach to a General View on Tolerances in Mechanical Engineering”. 2nd International Workshop on Integrated Product Development IPD 98. Magdeburg, Germany: pp. 65 - 76. 
Hu, M., Z. Lin, X. Lai and J. Ni, 2001, "Simulation and Analysis of Assembly Processes Considering Compliant, Non-ideal Parts and Tooling Variations”. International Journal of Machine Tools and Manufacture. Vol. 41 No. 15: 2233-2243.

Lee, J., Y. Long and S. J. Hu, "Robustness Evaluation for Compliant Assembly Systems". Proceedings of the 2000 ASME International DETC and CIE: Design for Manufacturing Conference, Baltimore, MD. Sep. 10-13, 2000.

Liu, S. and S. J. Hu, 1995, “An Offset Finite Element Model and its Application in Predicting Sheet Metal Assembly Variation", Intl. Journal of Machine Tools and Manufacture, Vol. 35, No.11, pp. 1545-1557.

Liu, S. and S. J. Hu, 1995, "Spot Weld Sequence in Sheet Metal Assembly: Its Analysis and Synthesis". ASME Manufacturing Science and Engineering. MED Vol. 2-2: 11451156.

Liu, S., S. J. Hu and T. C. Woo, 1996, “Tolerance Analysis for Sheet Metal Assembly”, Journal of Mechanical Design, Transactions of the ASME, Vol. 118, pp. 62-67.

Liu, S. and S. J. Hu, 1997, "Variation Simulation for Deformable Sheet Metal Assemblies Using Finite Element Methods". Journal of Manufacturing Science and Engineering, Transactions of the ASME, Vol. 119, pp. 368-374. 
Merkley, K. 1998. Tolerance Analysis of Compliant Assemblies. Ph.D. Dissertation, Brigham Young University, Provo, Utah.

Merkley, K., K. W. Chase and E. Perry, 1996, “An Introduction to Tolerance Analysis of Flexible Systems”, 1996 MSC World Users' Conference, Newport Beach, CA, June 1996.

Mortensen, A. 2002. A Methodology for Statistical Tolerance Analysis of Flexible Assemblies. M.S. Thesis, Brigham Young University, Provo, Utah.

Roark, R. 1965. Formulas for Stress and Strain.

New York, New York: McGraw-Hill Inc.

Sellem, E. and A. Rivière, 1998, “Tolerance Analysis of Deformable Assemblies”, Proceedings of DETC98: 1998 ASME Design Engineering Technical Conference, Atlanta, GA., 7 pages.

Sellem, E., C. A. de Hillerin, A. Clement and A. Rivière, 1999, "Validation of the Tolerance Analysis of Compliant Assemblies". Proceedings of DETC99: 1999 ASME Design Engineering Technical Conference, Las Vegas, NV, 6 pages.

Sellem, E., C. A. de Hillerin, A. Clement and A. Rivière, 1999, "Modelling-SimulationTesting of Compliant Assemblies". Global Consistency of Tolerances: Proceedings of 
the $6^{\text {th }}$ CIRP International Seminar on Computer-Aided Tolerancing. Enschede, Netherlands: pp. $355-364$.

Stout, J. 2000. Geometric Covariance in Compliant Assembly Tolerance Analysis. M.S. Thesis, Brigham Young University, Provo, Utah.

Soman, S. 1999. Functional Surface Characterization for Tolerance Analysis of Flexible Assemblies. M.S. Thesis, Brigham Young University, Provo, Utah.

Tonks, M. 2002. A Robust Geometric Covariance Method for Flexible Assembly Tolerance Analysis. M.S. Thesis, Brigham Young University, Provo, Utah. 


\section{Appendix}

\section{Production Sequence of Boeing Leading Edge Assembly}

Early in 2003 , the production sequence of the leading edge assembly was witnessed at Boeing Company factory in Salt Lake City. The following description was recorded shortly thereafter.

\section{A.1 Nomenclature}

To aid in the process explanation, the definition of parts, fixtures and tools used in the process is described.

Two types of parts make up the leading edge assembly, the flexible skin and the rigid ribs. The skin is flexible and can be deformed easily. It constitutes the outer surface of the assembly. Two ribs are used in the assembly to provide rigidity to the assembly. An additional tapered shim is added to the ribs. Because of this, the shim and ribs will be considered as one part.

Two fixtures constrain the parts from all movement when assembly takes place. The backbone fixture aids in the constraining and alignment of the two ribs. It also positions 
the skin for the installment of the clamshell fixture. The clamshell fixture constrains the skin in the desired shape and locates the rivet positions along the ribs.

Two sets of tools are required to produce the assembly. A step drill and drill bushing, fits in pre-defined holes in the clamshell fixture and is used to assign rivet locations. The second tool, a Cleco pin, is helpful in maintaining alignment in holes by expanding a small mandrel within them. Two types of Cleco pins are used in this process. Spring Clecos can be installed and removed quickly with the use of a specialized hand tool, and use internal compression springs to expand the mandrel. Draw-in Clecos are installed and removed with a pneumatic wrench, and use a screw thread to expand the mandrel. Draw-in Clecos provide a stronger hold than spring Clecos, but take more time to install properly.

All these tools and parts are shown on Table A.1 and will be as described in subsequent steps. In addition, a coordinate system is defined relative to the fixture, where the $x y$ plane corresponds to the flat part of the skin, the $x z$-plane corresponds to the horizontal datum of the fixture which the ribs sit upon, and the $y z$-plane corresponds to the edge of the skin as installed in the fixture. 
Table A.1: Boeing assembly definitions

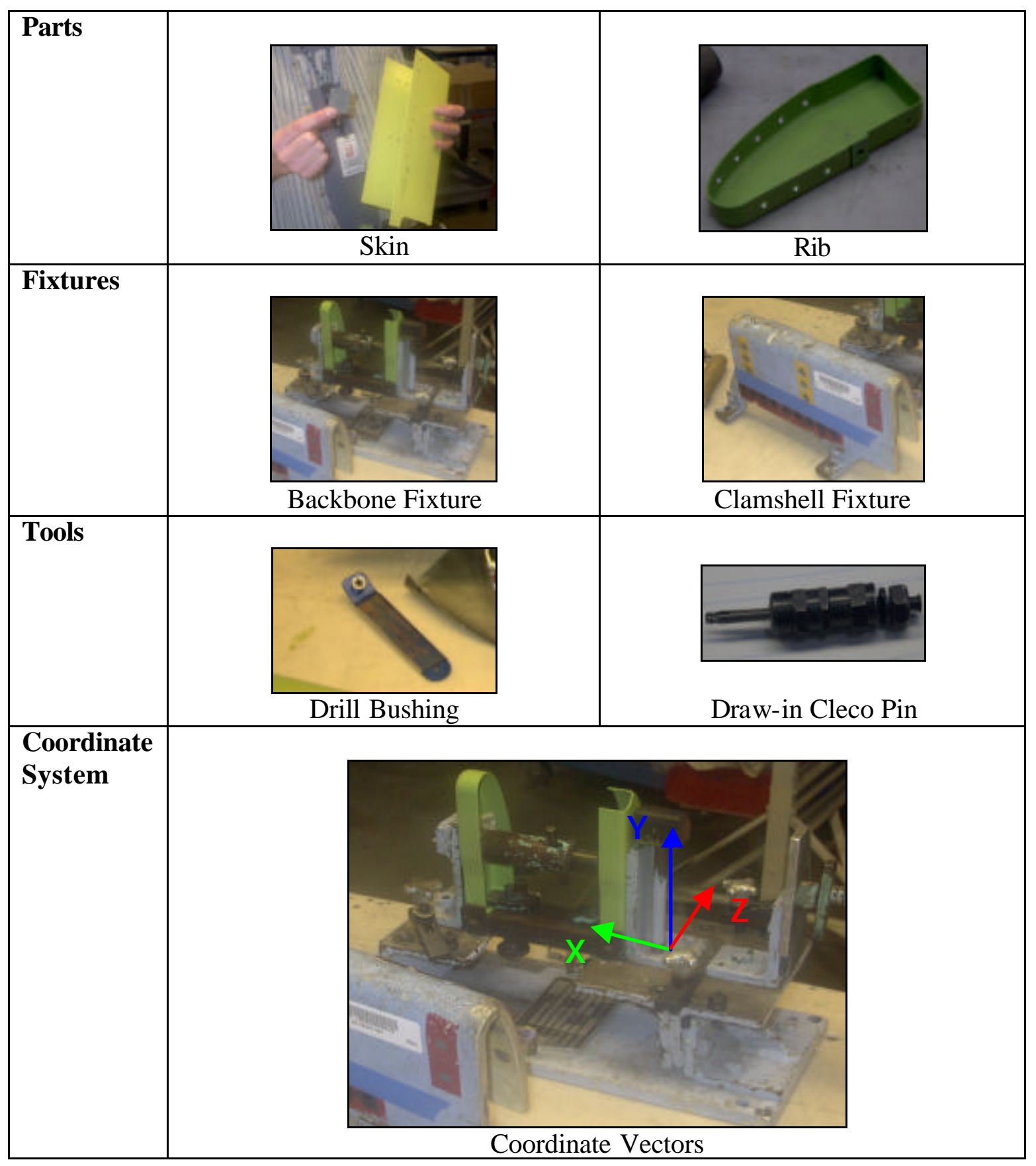




\section{A.2 Assembly Step 1-Rib Placement}

The ribs are installed into the backbone fixture against two vertical tabs defining the $x y$ plane, fixing movement in the $z$-direction. A spring-loaded pushrod pushes the ribs against two opposing vertical stops, thus preventing motion of the ribs in the $x$-direction. Additionally, each rib is clamped down to prevent movement in the $y$-direction.

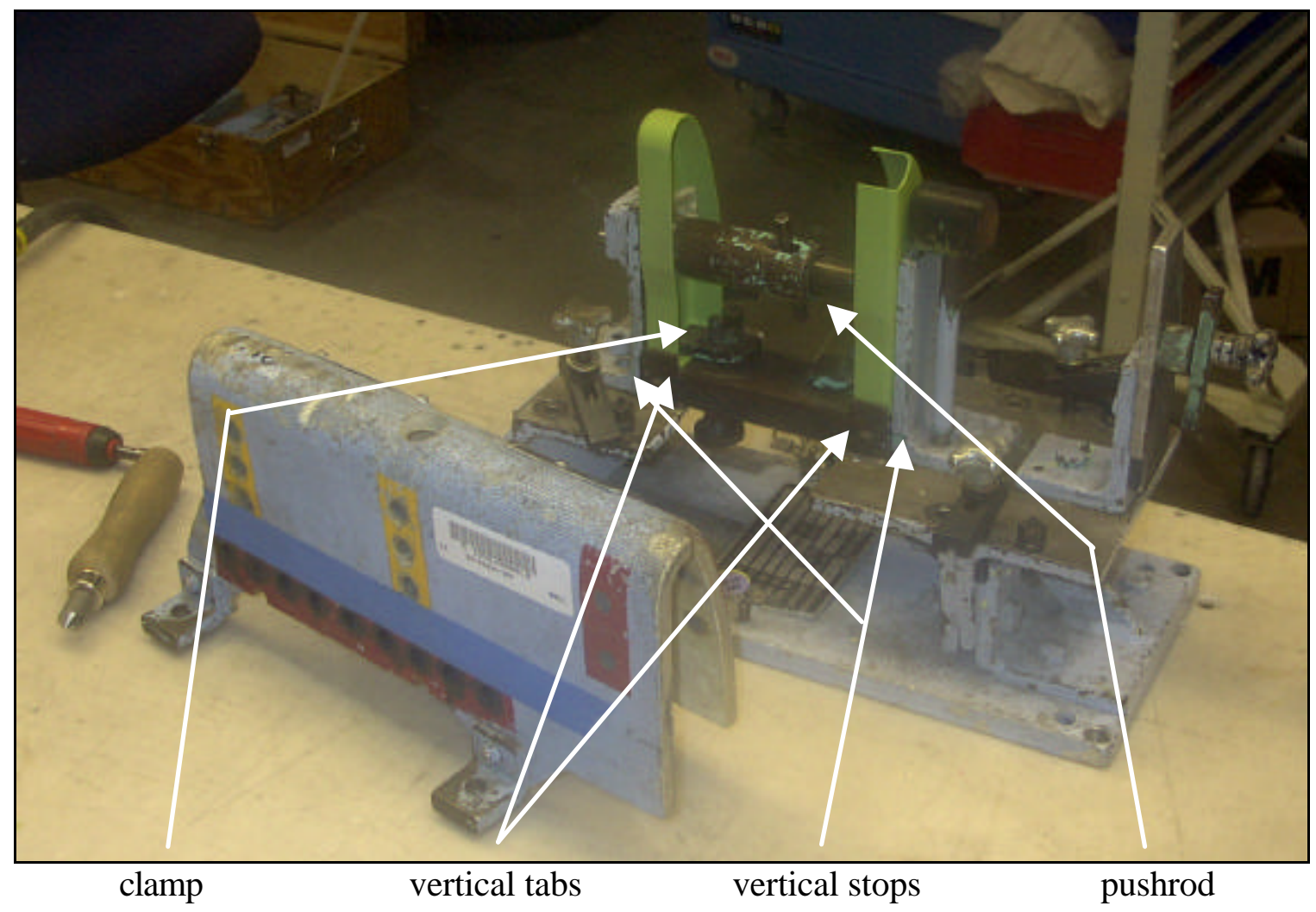

Figure A-1: The ribs inserted in the backbone fixture 


\section{A.3 Assembly Step 2 - Skin Placement}

The skin is positioned over the ribs and assembly. It is fixed against movement in the $y$ direction by two locator pins. Additionally, it is fixed against motion in the $x$-direction by a fixture tab.

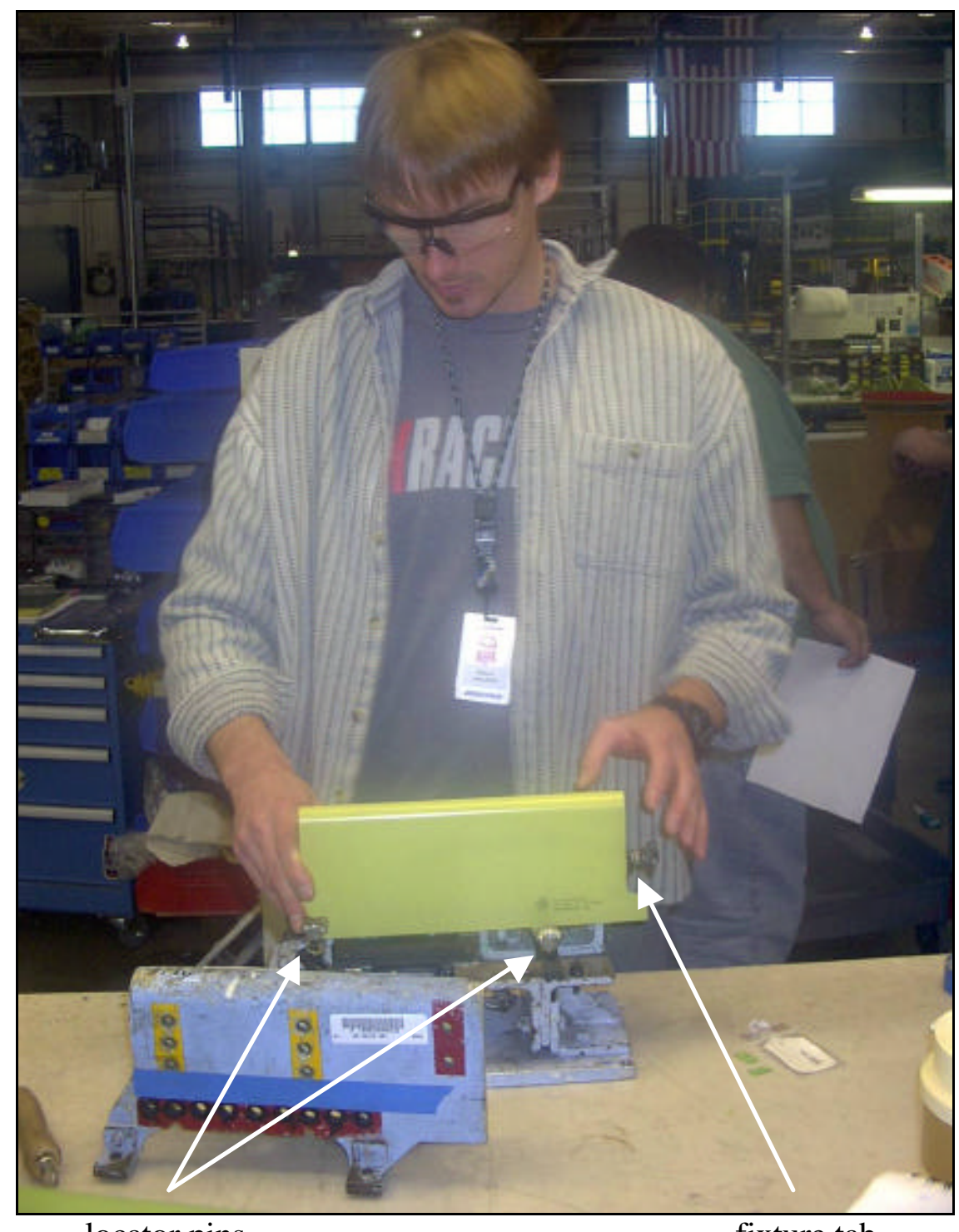

locator pins

fixture tab

Figure A-2: Positioning of the skin atop the ribs 


\section{A.4 Assembly Step 3 - Fixture Placement and Clamping}

The clamshell fixture is positioned over the skin. It is inserted into slots in the fixture, which make positioning the clamshell fixture easy. Four locating pins with clamps are used to position the clamshell fixture in the $x z$ - plane. The clamshell fixture is placed on these pins and then 4 hand-screws are screwed down to fully constrain it. A stop at the edge of the fixture is used to fix the clamshell position in the $x$-direction.

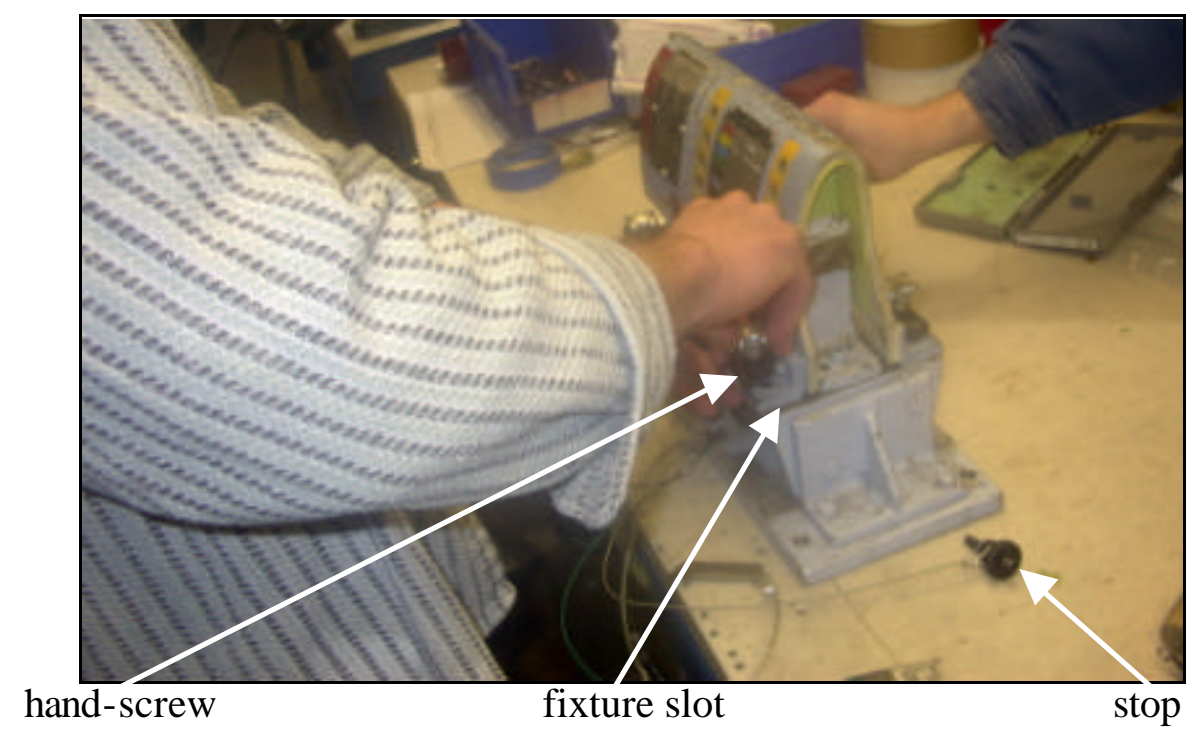

Figure A-3: Clamping of the clamshell fixture over the skin and ribs

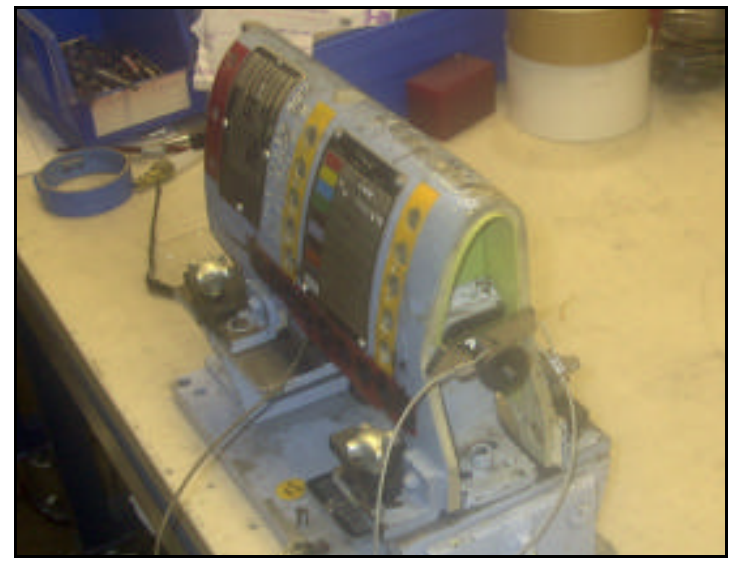

Figure A-4: Final clamping 


\section{A.5 Assembly Step 4-Hole Drilling 1}

The holes along the front are then drilled using a $\varnothing 0.373 "$ drill bushing and a $\varnothing 0.216 "$ step drill. Spring Clecos are used to align mating holes. The pattern for drilling and affixing Clecos is diagrammed in Figure A-6. A Cleco pin is installed immediately after drilling each corner hole, but not installed in the intermediate holes.

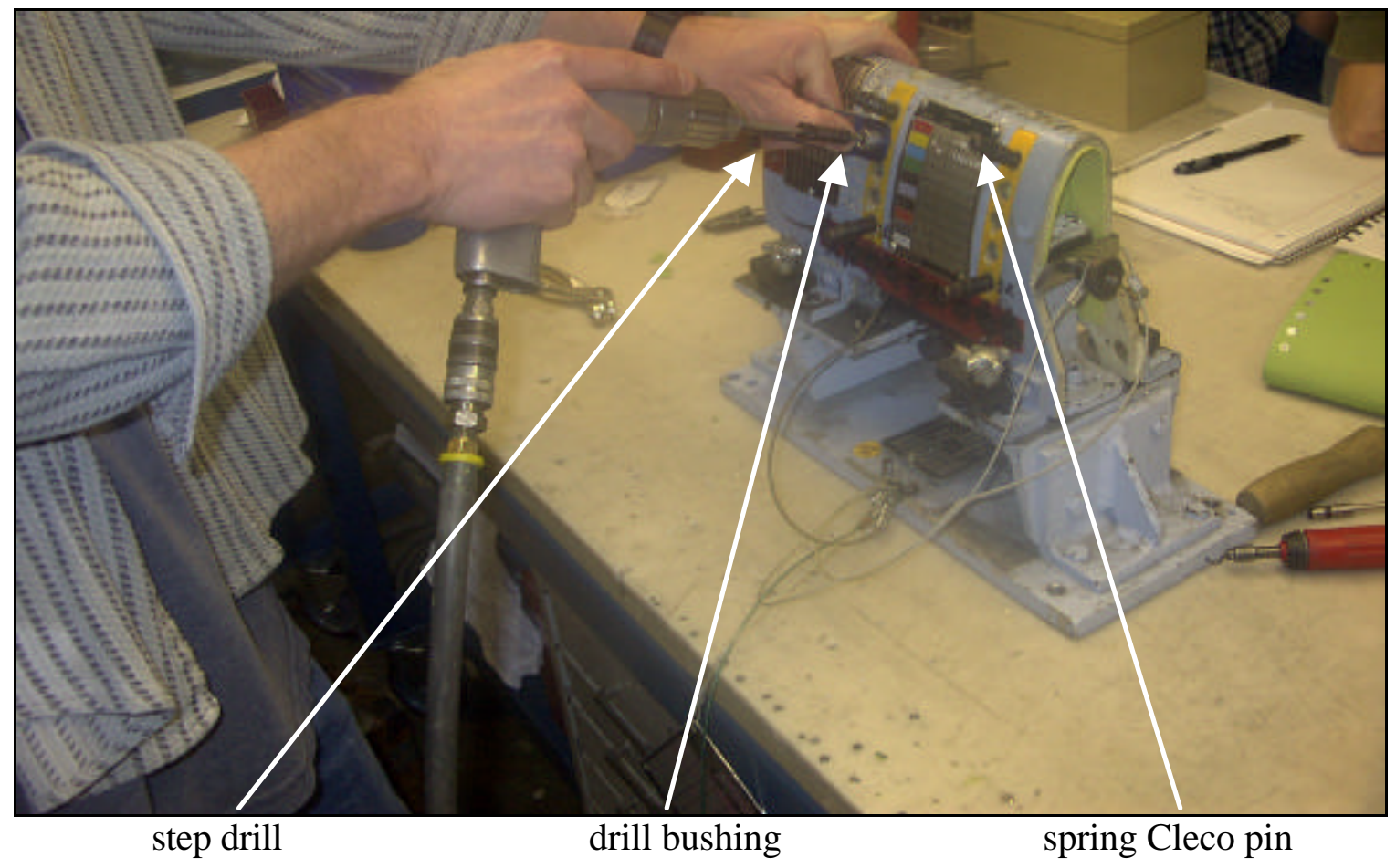

Figure A-5: Drilling and Cleco pin installation

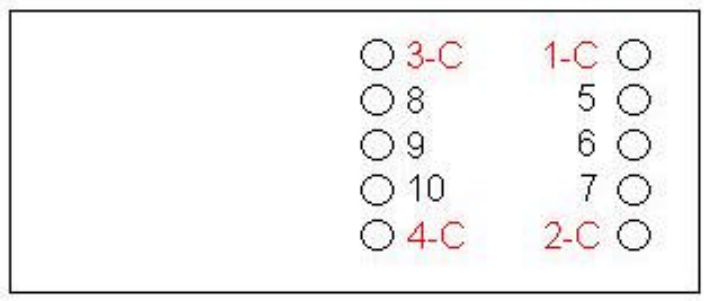

Figure A-6: Figure showing the drilling and Cleco pin installation sequence 


\section{A.6 Assembly Step 5-Hole Drilling 2}

The holes along the back are drilled in the same manner as the front holes using a $\varnothing 0.373 "$ drill bushing and a $\varnothing 0.216 "$ step drill. The pattern for drilling and affixing spring Clecos is diagramed in Figure A-8. Additionally, tentative hole locations are marked in pen (but not drilled) upon the part for use in subsequent assembly steps.

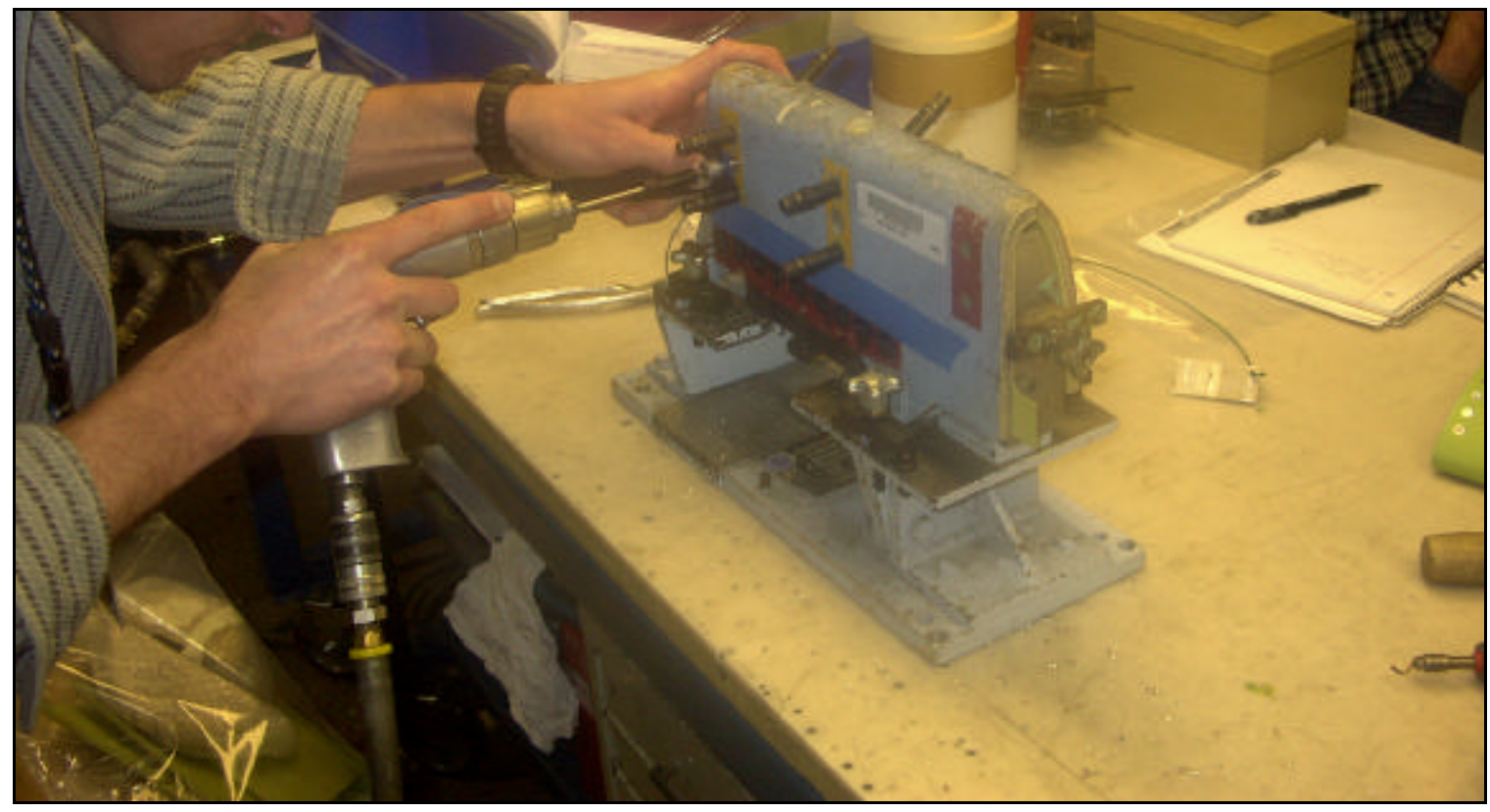

Figure A-7: Figure showing the drilling and Cleco pin installation sequence

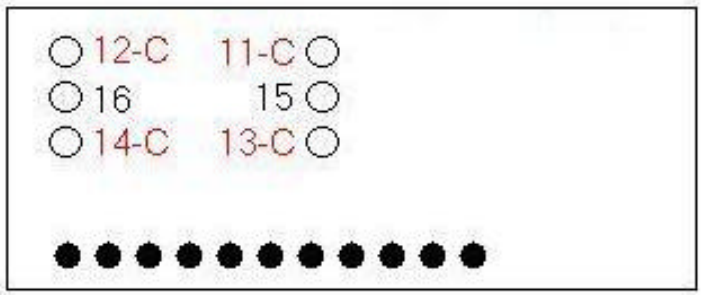

Figure A-8: Figure showing the drilling and Cleco pin installation sequence, as well as the pen marking pattern 


\section{A.7 Assembly Step 6-Hole Filing}

The assembly is removed from the fixtures and disassembled to the individual

components. The drilled holes on both the ribs and the skin are filed inside and out using a flat, coarse file to remove burrs.

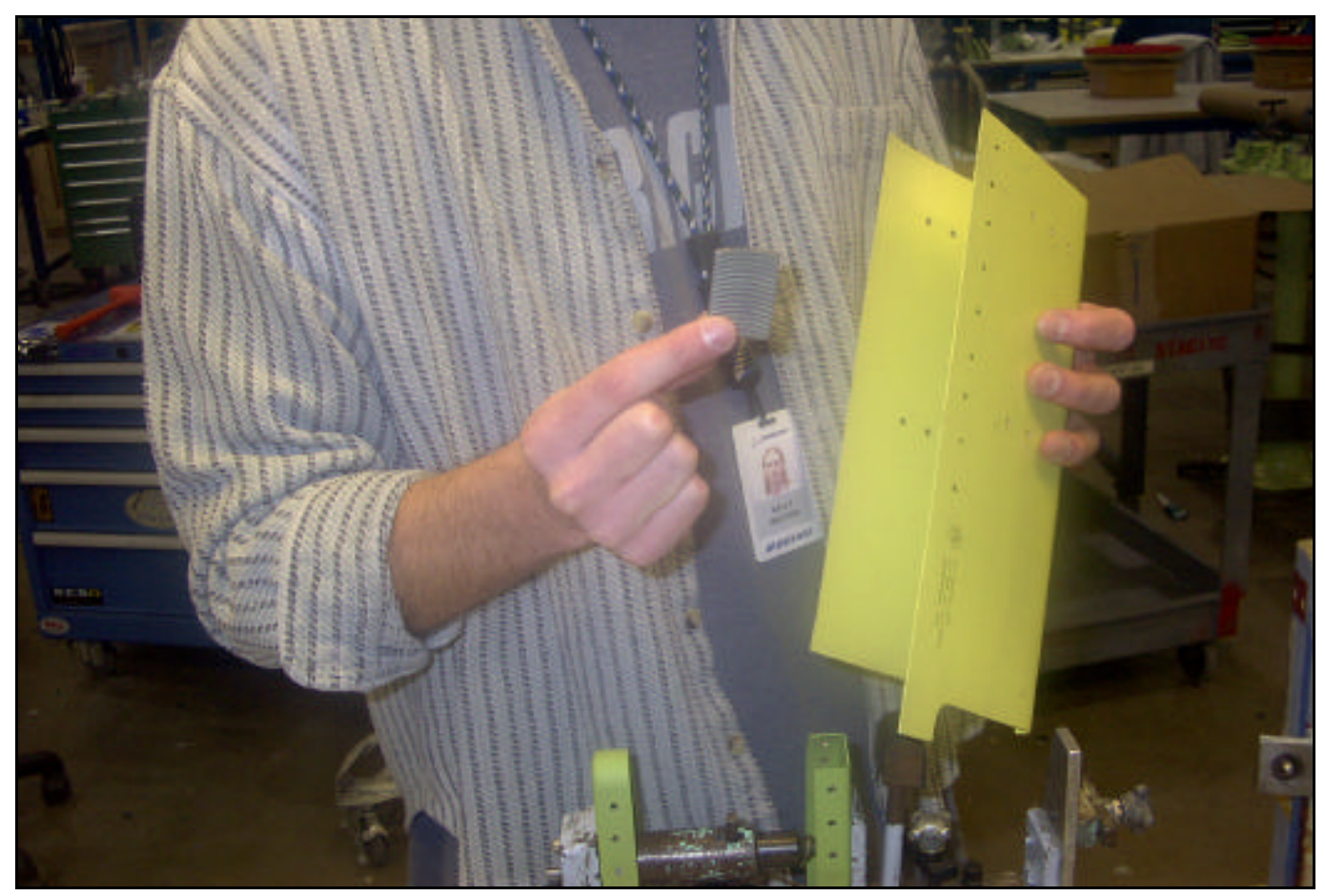

Figure A-9: All component holes are filed to remove burrs 


\section{A.8 Assembly Step 7-Tapered Shim Installation}

A tapered shim with a pre-drilled hole is affixed to the back of both ribs, where a relief is manufactured into the part. This shim is aligned by a straight rule to the flat of the rib and affixed with adhesive. A shim is affixed to both ribs.

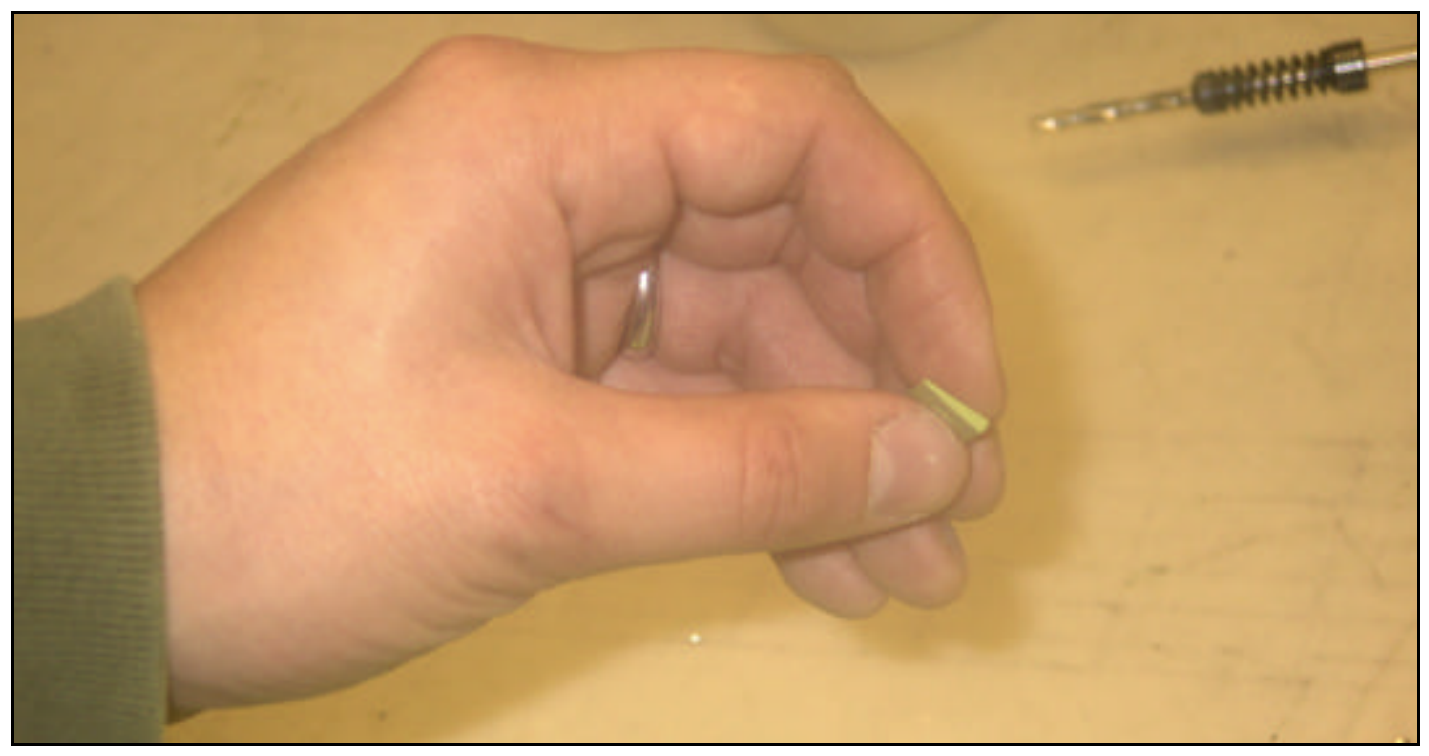

Figure A-10: The tapered shim
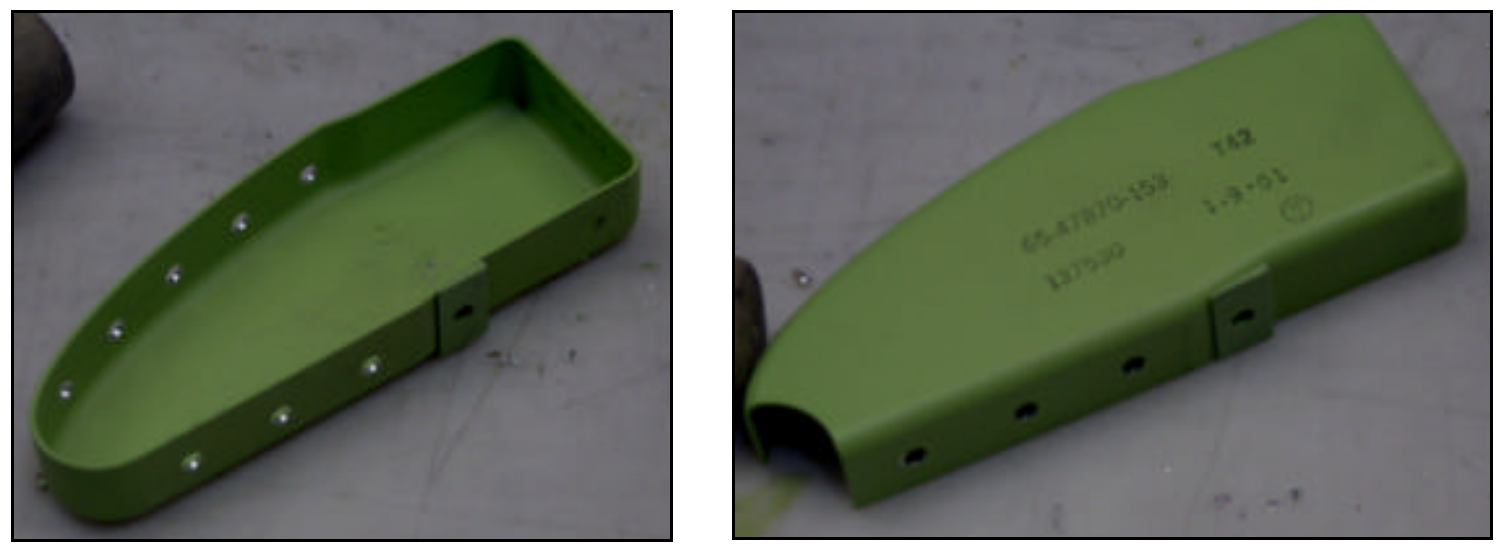

Figure A-11: A tapered shim affixed to both ribs 


\section{A.9 Assembly Step 8-Sealant Preparation}

The sealant is prepared by inserting the catalyst into the sealant cartridge and mixing vigorously for 2 minutes. The mixture has the consistency of hot glue afterwards. Upon mixing, the sealant must be used within a few hours. The sealant is loaded in a pneumatic gun to aid in the application.

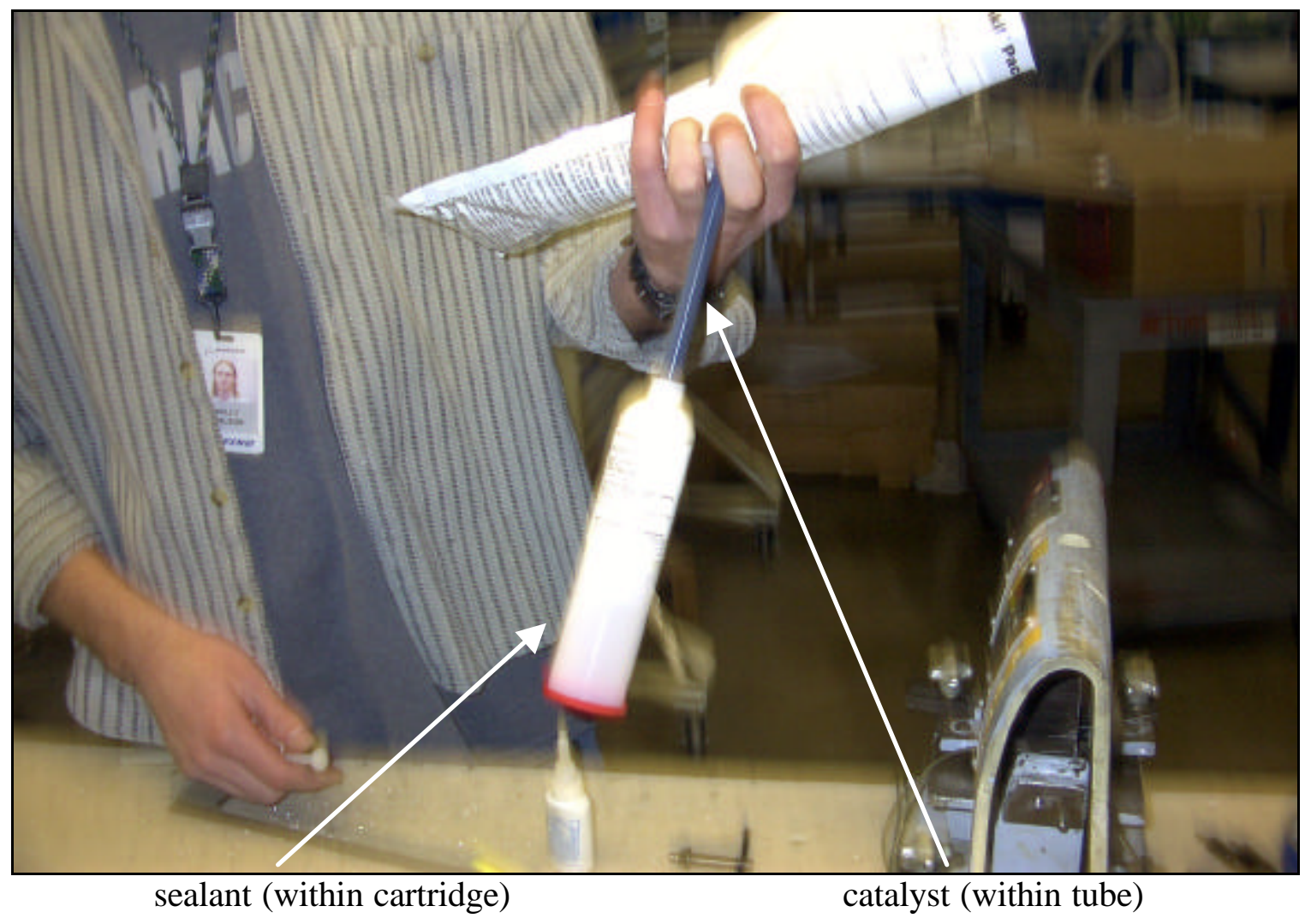

Figure A-12: Preparation of the sealant 


\section{A.10 Assembly Step 9-Component Preparation}

The mating surfaces of the ribs and skin are cleaned and degreased using denatured alcohol, in preparation for the sealant.

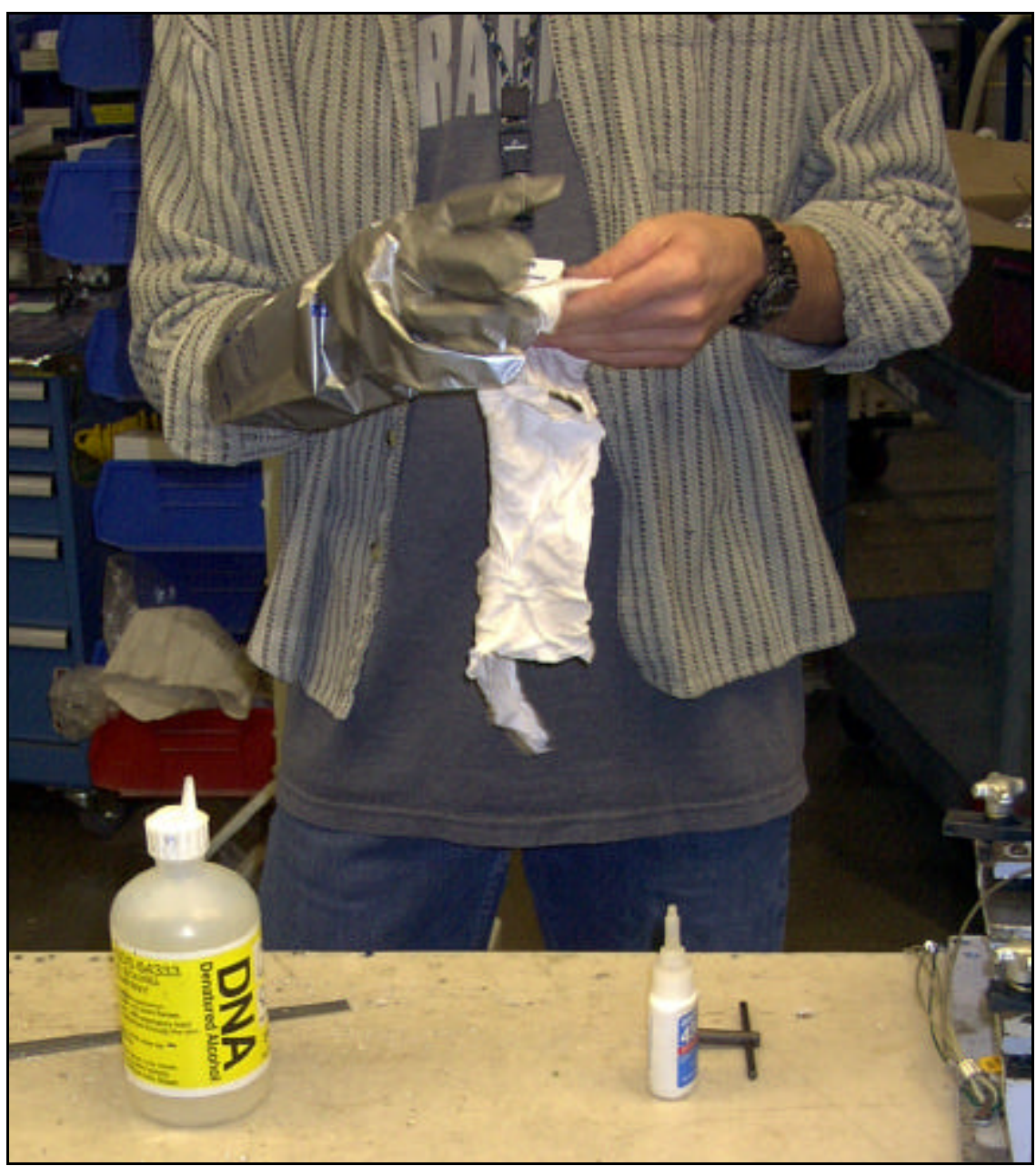

Figure A-13: Cleaning of the parts with denatured alcohol 


\section{A.11 Assembly Step 10 - Final Hole Drilling}

The final hole is drilled into the rib by hand using the hole in the tapered shim as a guide.

This is done to both ribs. The drilled hole is filed like the other ones.

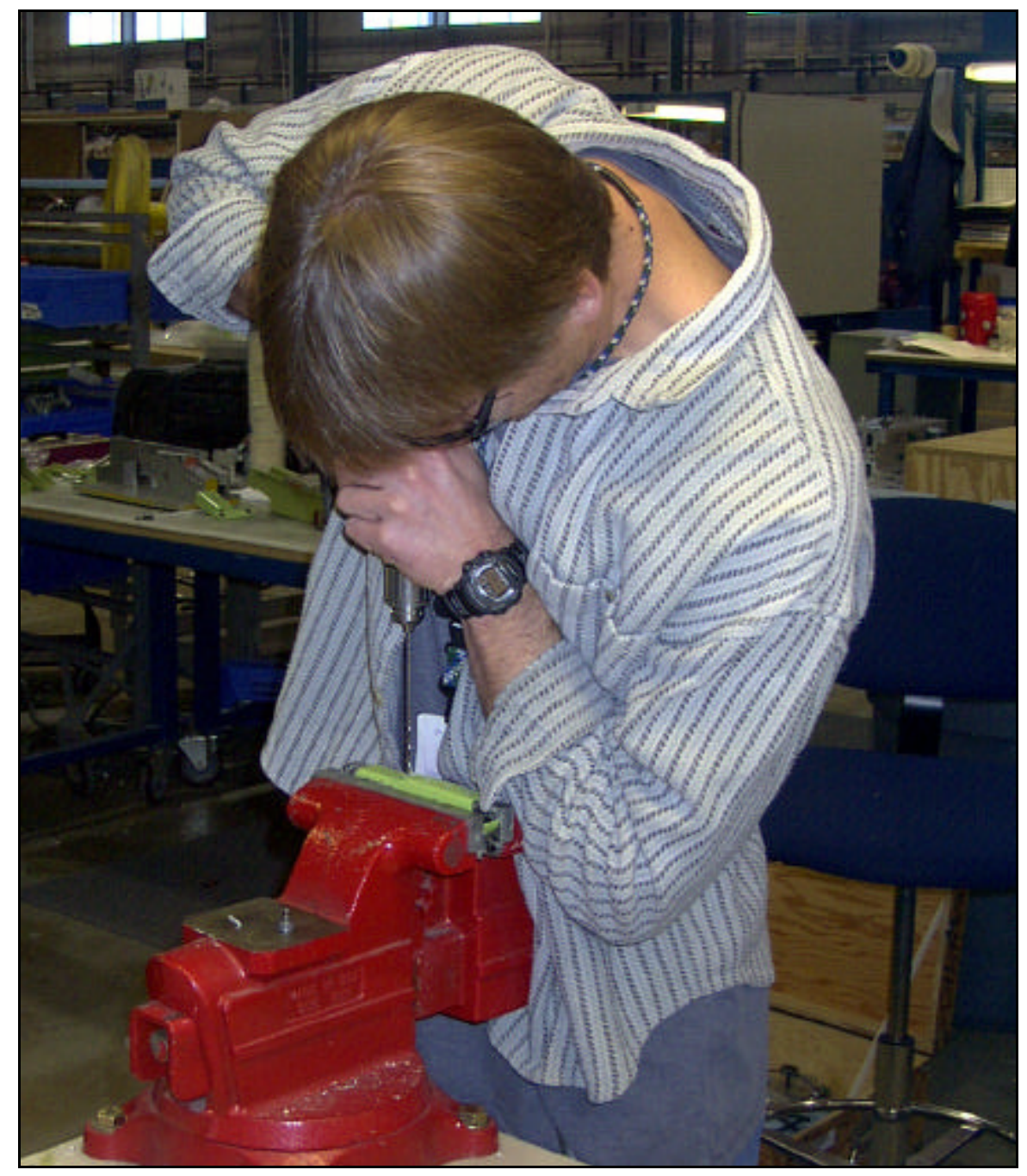

Figure A-14: Drilling of the final hole in the ribs 


\section{A.12 Assembly Step 11 - Sealant Application}

The sealant is applied to the contact surfaces of the ribs and spread evenly. The ribs and

skin are then affixed by hand and sight. The drilled holes are pre-aligned by the operator.

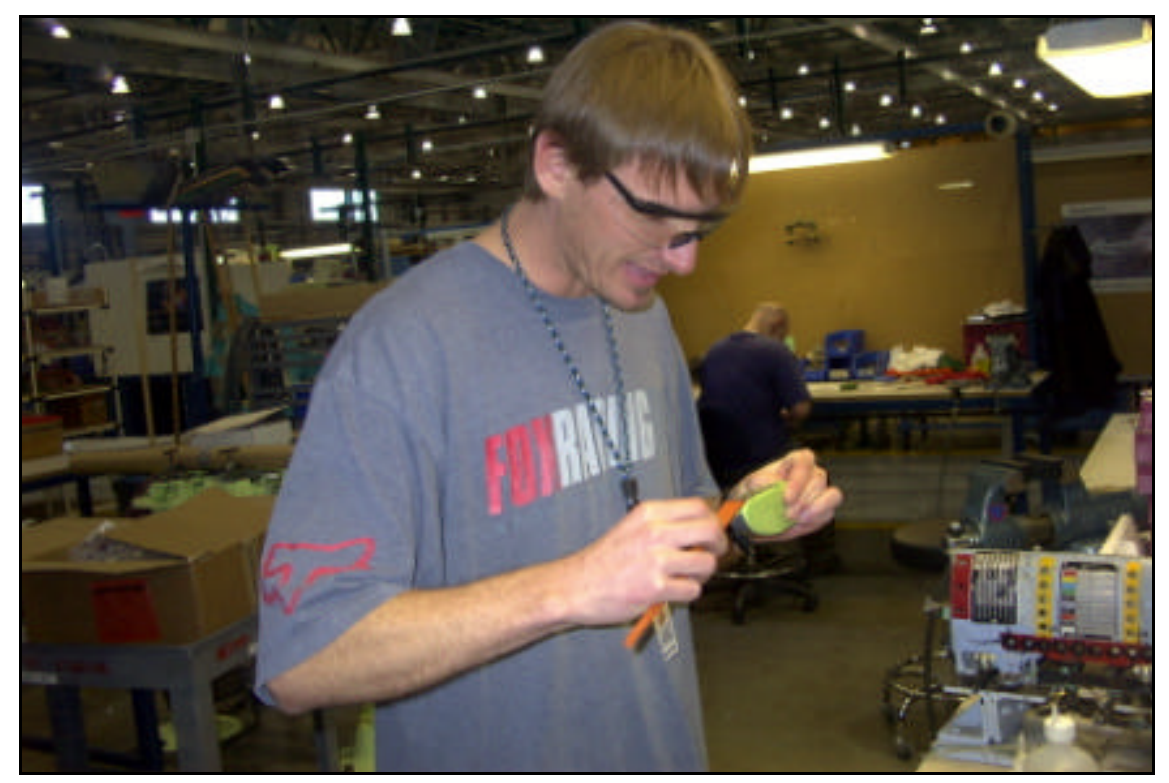

Figure A-15: Application of the sealant to a rib

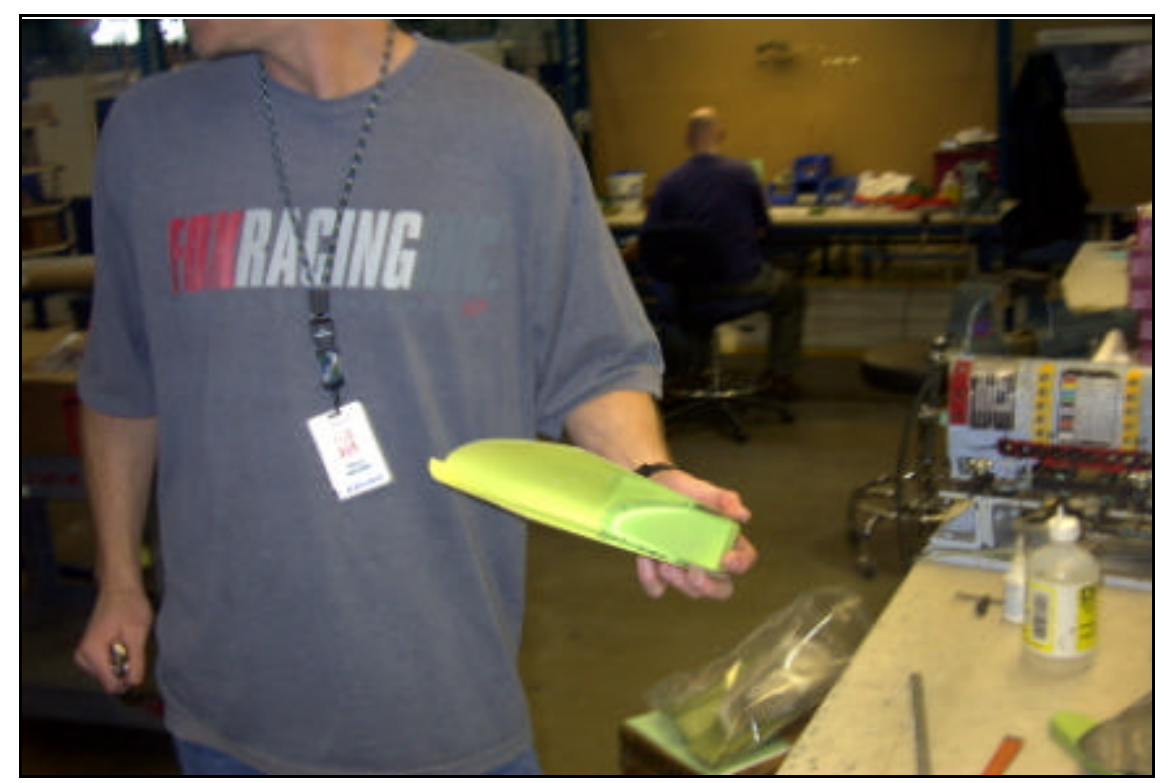

Figure A-16: Pre-alignment of rib and skin by operator 


\section{A.13 Assembly Step 12 - Spring Cleco Pin Insertion}

Spring Clecos are reinstalled into the corner holes to align the rib and skin holes. The spring Clecos are installed into the same holes as in steps 4 and 3. The Clecos are inserted in both the front and back of the assembly.

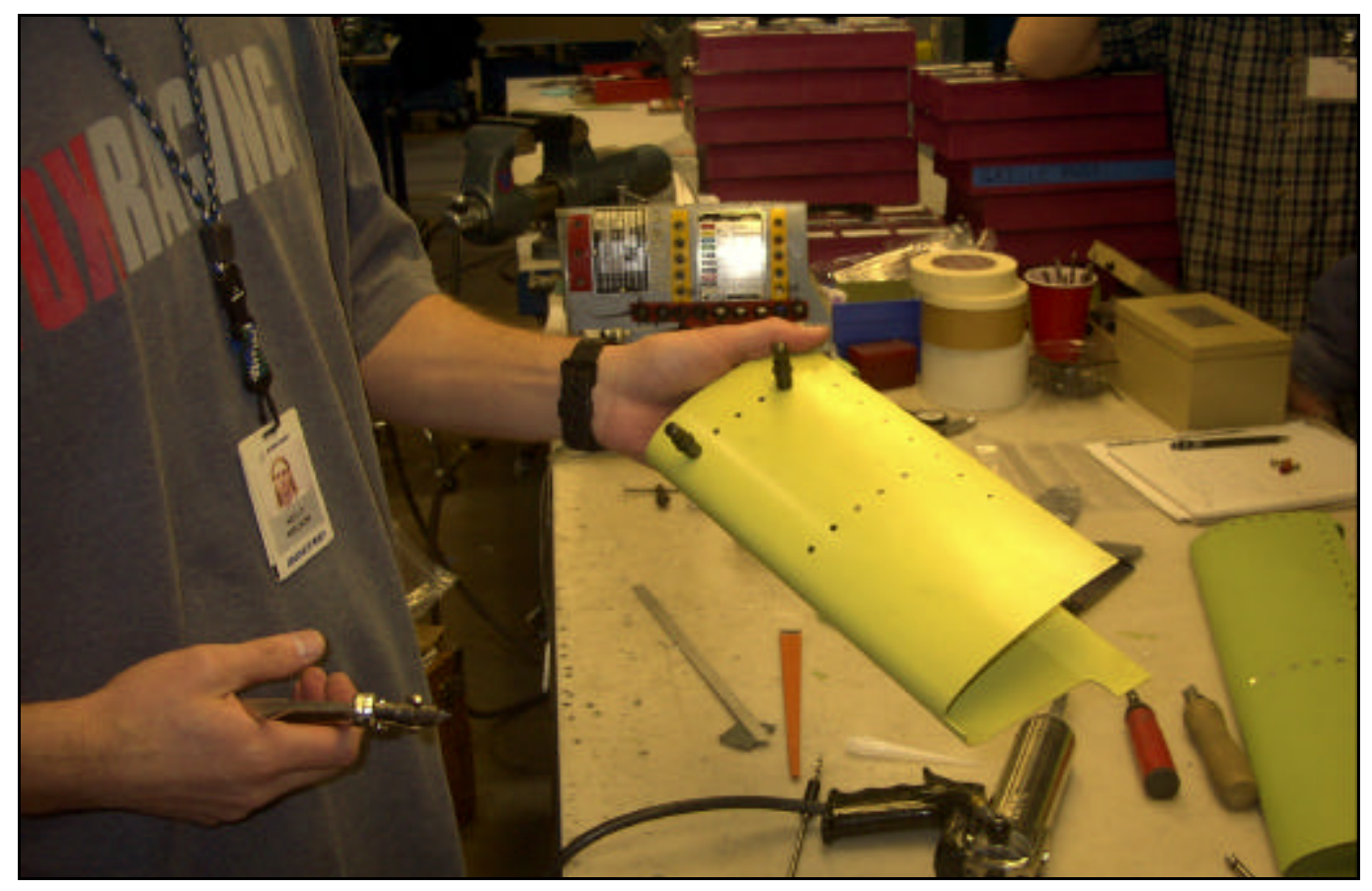

Figure A-17: Installation of spring Clecos 


\section{A.14 Assembly Step 13 - Draw-in Cleco Pin Insertion}

Draw-in Clecos are installed into all the remaining holes and tightened with a pneumatic torque wrench. Once these are installed, all spring Clecos from the previous step are pulled out and replaced with draw-in Clecos.

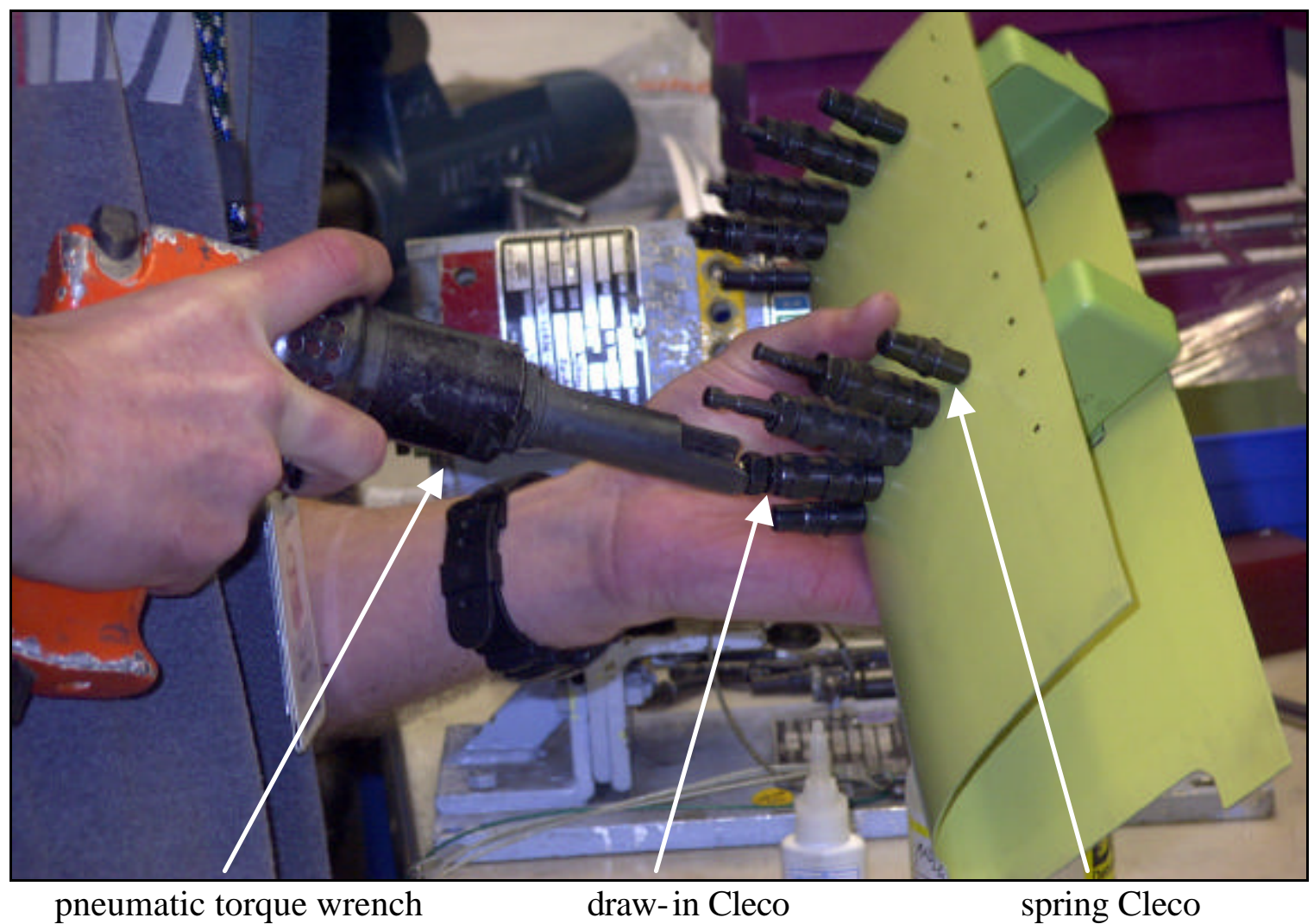

Figure A-18: Pre-alignment of rib and skin by operator 


\section{A.15 Assembly Step 14 - Sealant Curing}

Two clamps are installed to put pressure on the shims. The assembly is left in this position for 24 hours to cure the sealant.

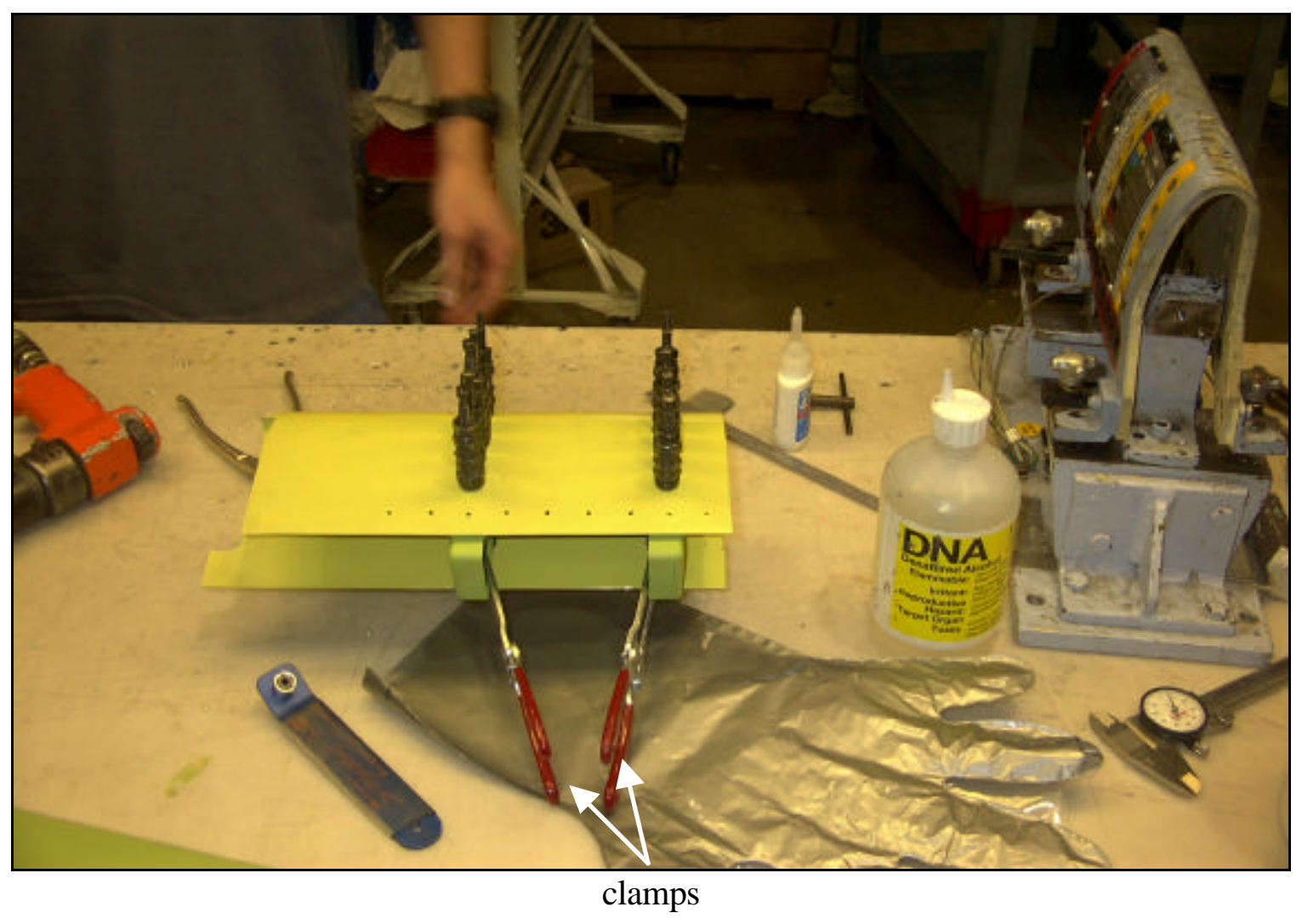

Figure A-19: Clamping of assembly to cure sealant 


\section{A.16 Assembly Step 15 - Rivet Installation}

After the sealant has cured, an anvil in a vise is used to affix the rivets. A draw-in Cleco pin is taken out, the assembly is positioned over the anvil, and the rivet is driven against the anvil using an air hammer. This process was not demonstrated to us during our trip.

Once all the rivets are installed, the subassembly is ready for further production steps.

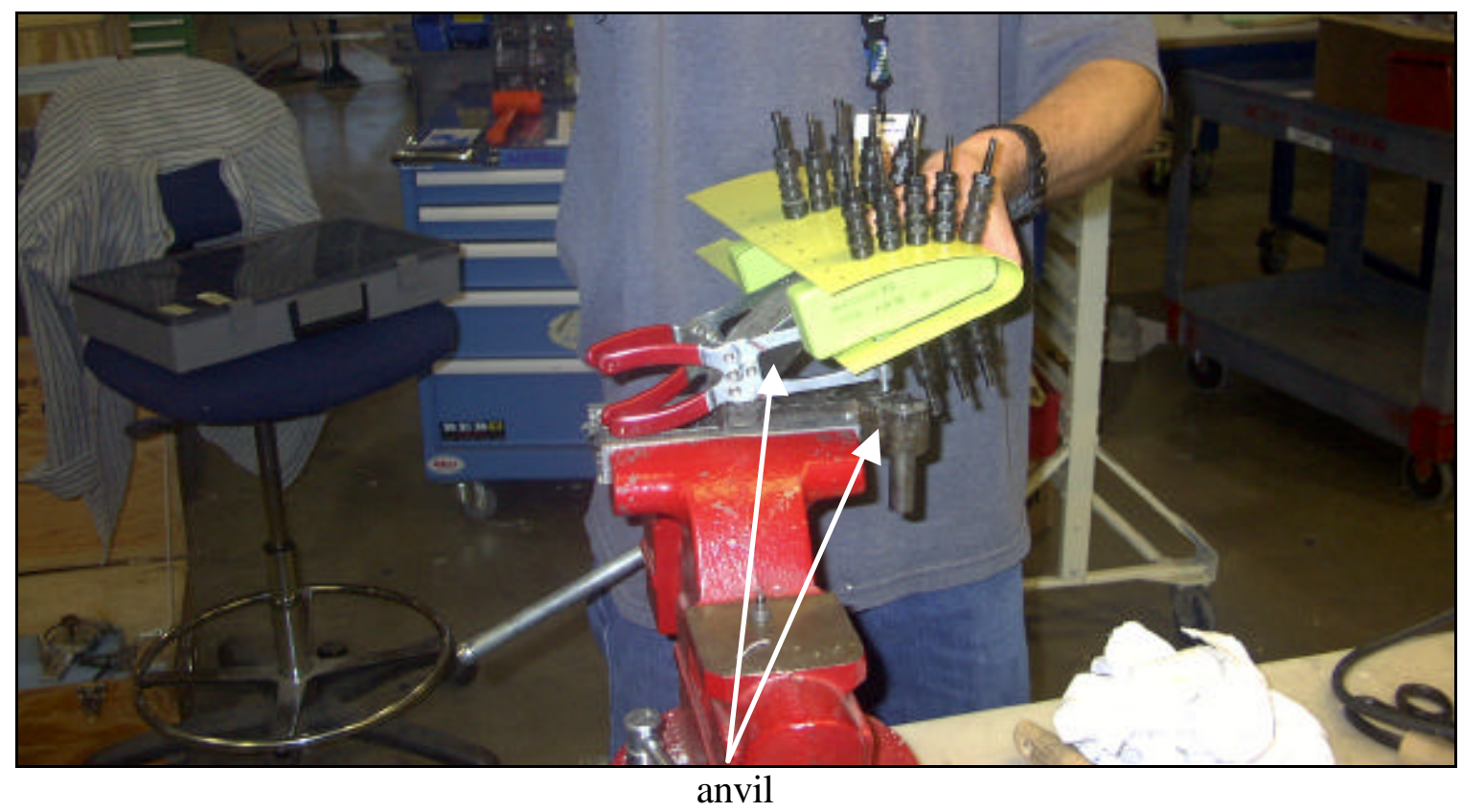

Figure A-20: Procedure to rivet assembly together 


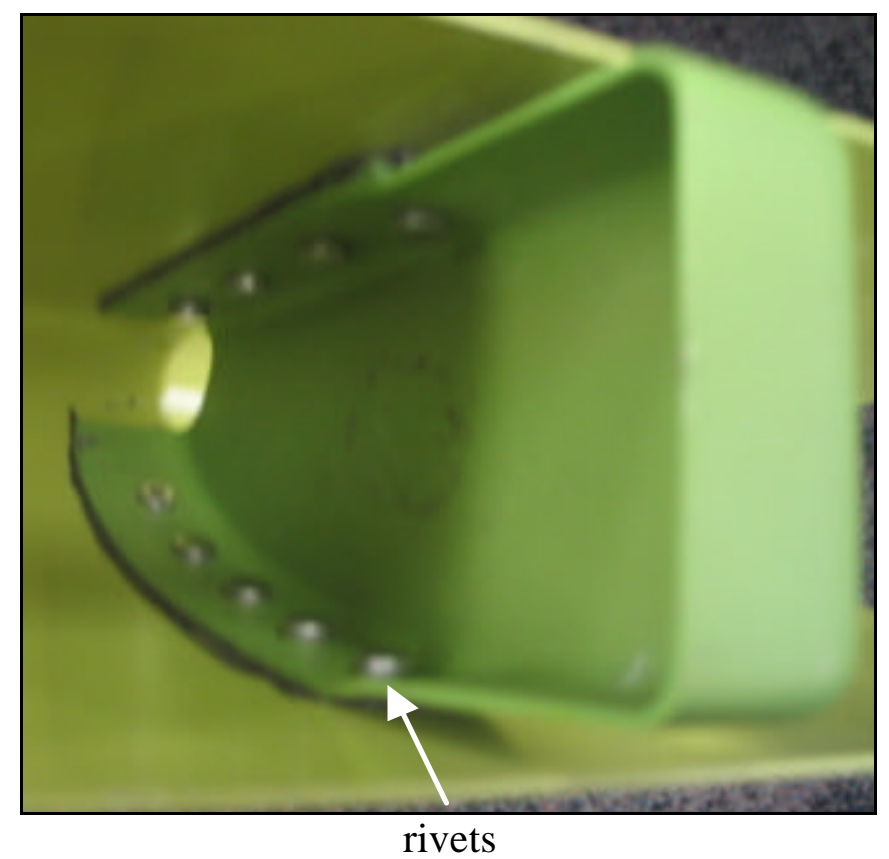

Figure A-21: Assembly showing finished rivet heads 